\title{
Performance of Post-Tensioned Timber and Engineered Timber Adhesives with Fire Exposure
}

\author{
by \\ Hailey Quiquero
}

A thesis submitted to the Faculty of Graduate and Postdoctoral Affairs in partial fulfillment of the requirements for the degree of

\author{
Master of Applied Science \\ in
}

Civil Engineering

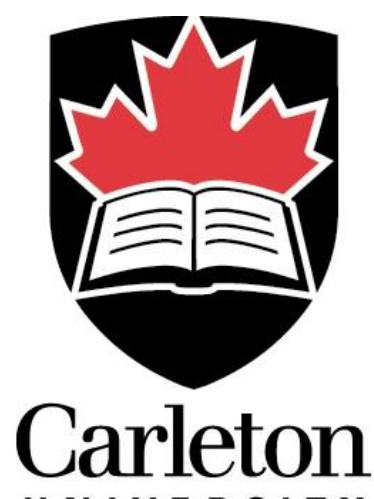

U N I E R S I T Y

Ottawa, Ontario 


\section{Abstract}

An increased environmental conscientiousness in society and the abundance of timber in Canada has inevitably lead to the desire for more timber construction. In order to increase the opportunity for timber products in construction, novel building systems such as PostTensioned (PT) timber are required. A model was developed and validated in Abaqus to investigate the ability of FEM software packages to model PT timber in fire conditions. The numerical model showed highly promising results for demonstrating the loading and failure behaviour of PT timber beams. Beam failure times were modelled within $5 \%$, and load-deflection behaviour and failure mechanisms were accurately demonstrated. Additionally, the performance of timber adhesives after fire damage was examined after previous research suggested further investigation was required. Based on the experimental results, additional zero-strength layer thicknesses were estimated conservatively to be 23 $\mathrm{mm}$ beyond the char front $\left(95^{\text {th }}\right.$ percentile) to account for the loss of strength (subject to various limitations). It is recommended that a new standardized test be developed for timber adhesives which quantifies the performance beyond the char layer in burnt engineered timber so that individual adhesives may be evaluated. 


\section{Acknowledgements}

First and foremost, I must express my utmost appreciation and thanks to my supervisor, Dr. John Gales for his tireless assistance and guidance throughout my time working with him. My research began with John as an undergraduate student and his countless hours of work and support have helped me grow into the researcher I am today. His encouragement always pushed me to new limits and his support of all students is inspiring.

I would also like to express my appreciation for the time and suggestions from Ata Khan, David Lau, Nourredine Bénichou and Magdi Mohareb, which elevated my thesis to the best work I could produce.

I also must acknowledge many of my colleagues at Carleton who I have worked with throughout the degree including Lauren Folk, Claudia Gaudreault, Seth Gatien, Arlin Otto, Josh Woods and Matthew Smith. I thank you for the endless amounts of advice, learning, travelling, laughter and conferences attended. I must especially thank Matthew Smith for guiding me in learning about consultancy and how my research could benefit practitioners. I would also like to thank the Carleton University Civil lab staff, especially Stanley Conley and Jason Arnott, for their help and guidance with running my experiments.

I would also like to thank Dr. Anthony Abu for graciously accepting me into his team at the University of Canterbury and teaching me not only about PT timber and numerical modelling, but also invaluable lessons on life as a graduate student and where best to travel in NZ. Furthermore, thank you to Dr. Peter Moss and Dr. Andy Buchanan for their guidance. I will forever appreciate and look back fondly on my time at UC. 
Additionally, I give my thanks to my colleagues at UC, especially Elizabeth North and Alex Meredith, for welcoming me into their lives as I endeavored to feel at home in NZ.

Finally, I must acknowledge the abundance of support I have received from my loved ones. To Chad, thank you for supporting me in this post-graduate endeavor and especially in spending several months abroad, coming to visit and keeping me sane through countless challenges and breakthroughs. To my parents Mario and Susan and my sister Lauren, thank you for your continuous support and for making me feel that I can accomplish anything I set my mind to.

I would also like to acknowledge Carleton University, NSERC (CGS and MSFSS) and the OGS program for supporting me throughout my degree. 


\section{Table of Contents}

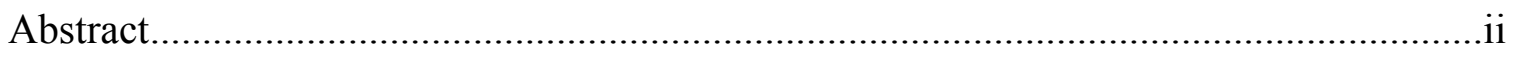

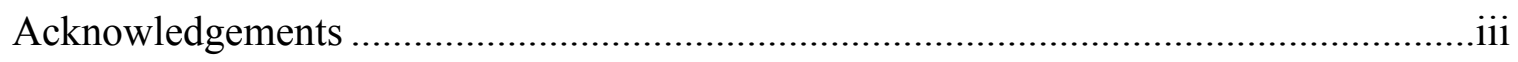

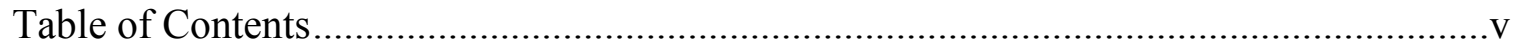

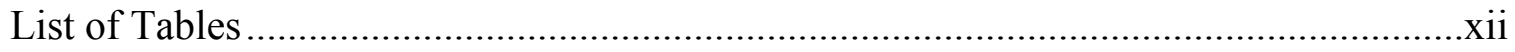

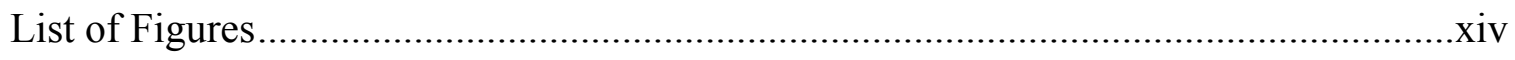

Statement of Co-Authorship and Scholarly Outputs ......................................................xv

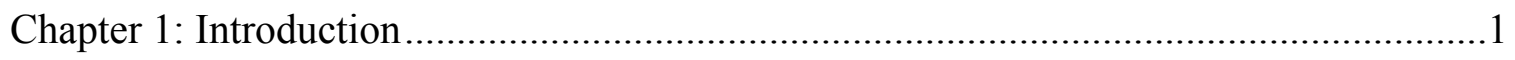

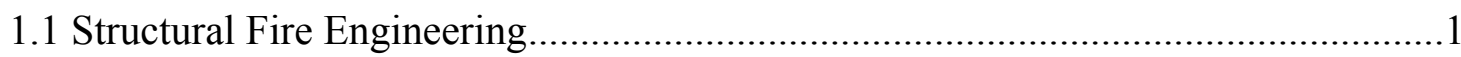

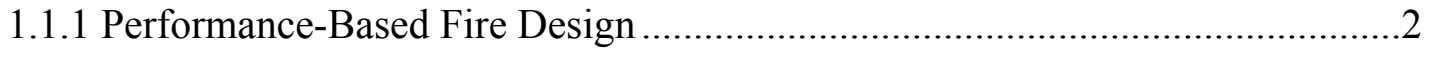

1.1.2 Structural Fire Engineering in Canada ...........................................................

1.1.3 Building and Innovating with Timber ...........................................................

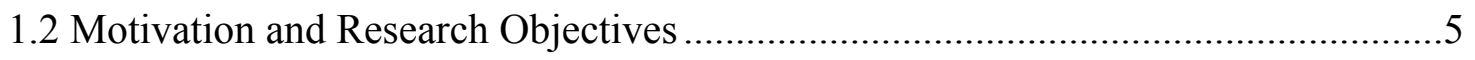

1.3 Research Scope and Thesis Outline ................................................................6

1.3.1 Chapter 2 - Background and Literature Review ............................................

1.3.2 Chapter 3 - Finite Element Modelling of Post-Tensioned Timber Beams .........7

1.3.3 Chapter 4 - Timber Adhesive Performance after Fire Damage ..........................

1.3.4 Chapter 5 - Conclusions and Recommendations .............................................8

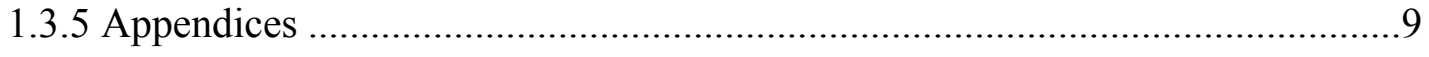

Chapter 2: Background and Literature Review ............................................................10 


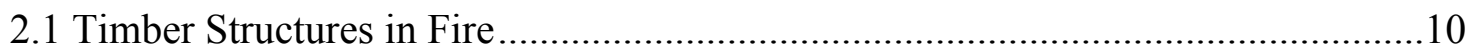

2.1.1 Engineered Timber Products .......................................................................12

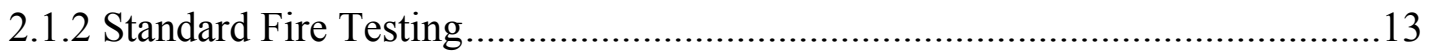

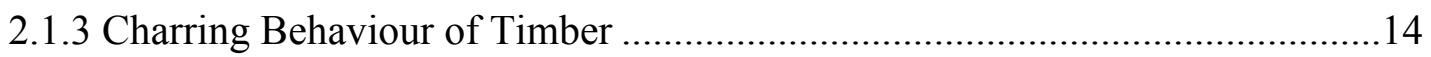

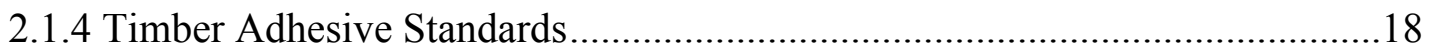

2.1.5 Research on Timber Adhesive Fire Performance ............................................20

2.2 Numerical Modelling of Timber in Fire Conditions................................................22

2.2.1 Past Numerical Modelling of Timber ...........................................................29

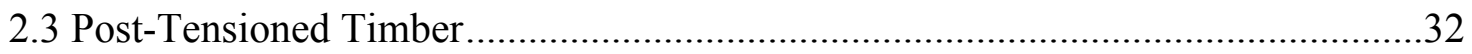

2.3.1 Post-Tensioned Timber Beams........................................................................

2.3.2 Past Experiments on PT Timber in Fire …………..........................................34

2.3.3 Existing and Proposed PT Timber Structures.................................................38

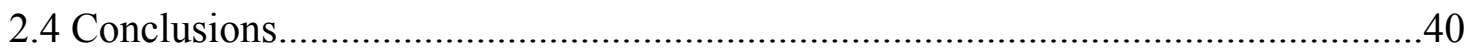

Chapter 3: Finite Element Modelling of Post-Tensioned Timber Beams ...........................42

3.1 Introduction

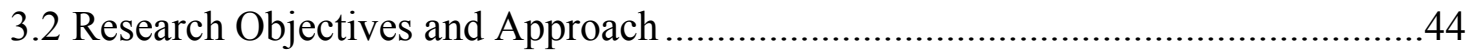

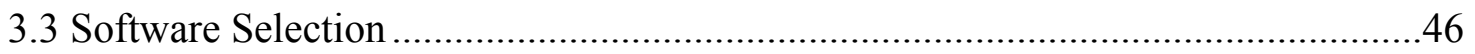

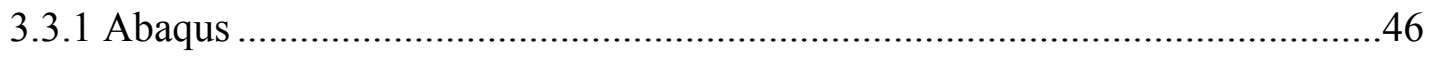

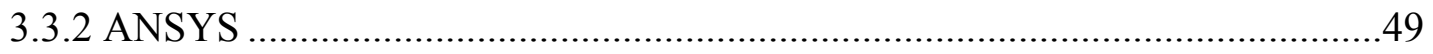

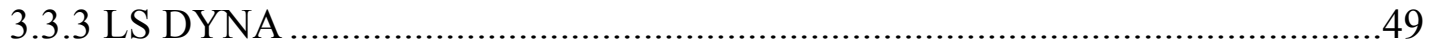




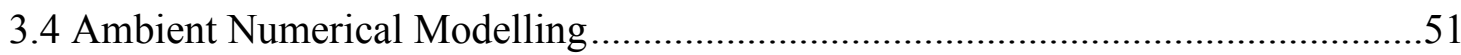

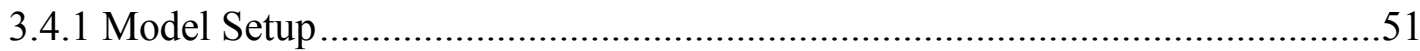

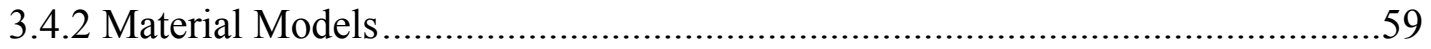

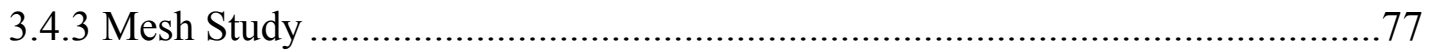

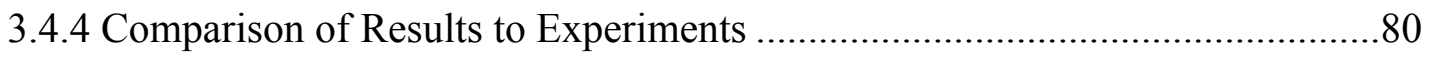

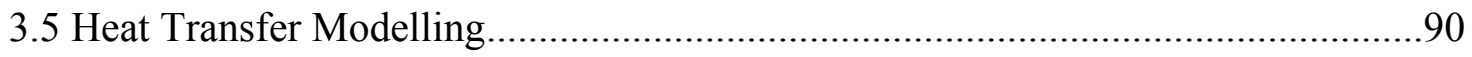

3.5.1 Model Setup ……………………………………......................................91

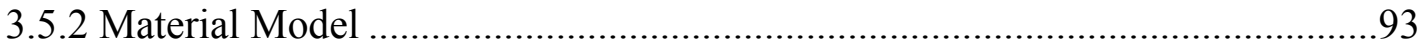

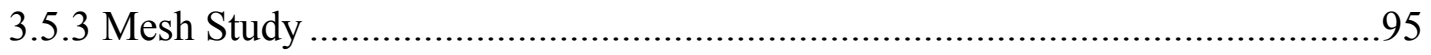

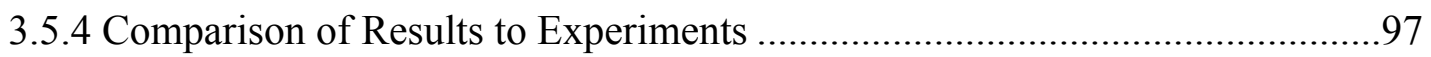

3.6 Sequentially Coupled Thermal-Mechanical Analysis ...........................................103

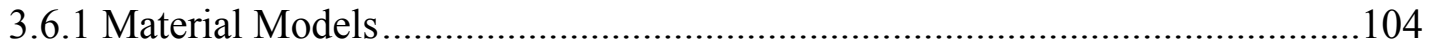

3.6.2 Mesh Study ………………………………...........................................111

3.6.3 Comparison of Results to Experiments .......................................................113

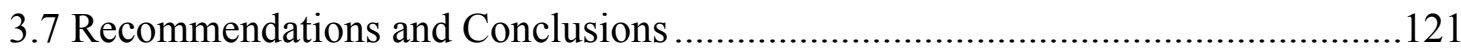

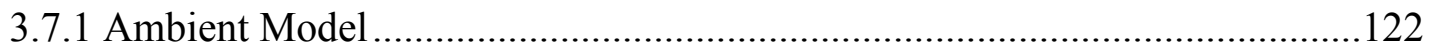

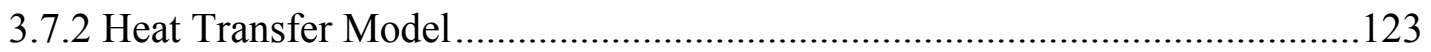


3.7.3 Thermal-Mechanical Model

Chapter 4: Timber Adhesive Performance after Fire Damage ........................................125

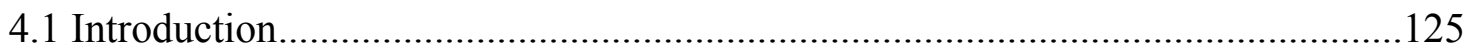

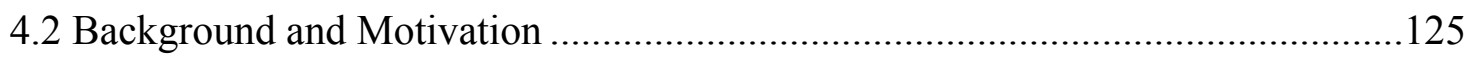

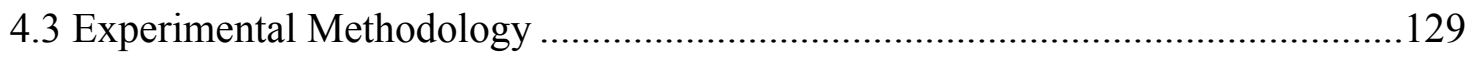

4.3.1 Cone Calorimeter Heating ..........................................................................131

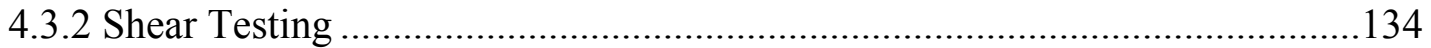

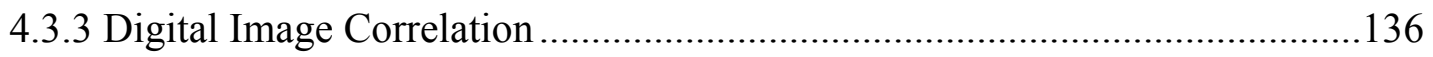

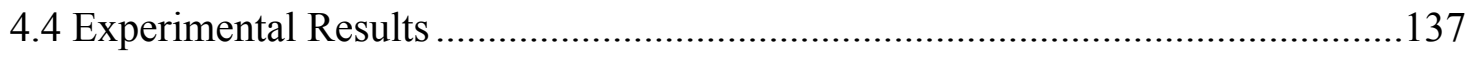

4.4.1 Heating Observations and Char Results .......................................................138

4.4.2 Adhesive Strength Capacity ......................................................................141

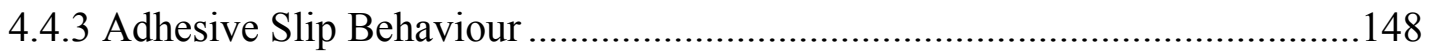

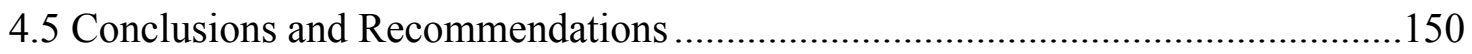

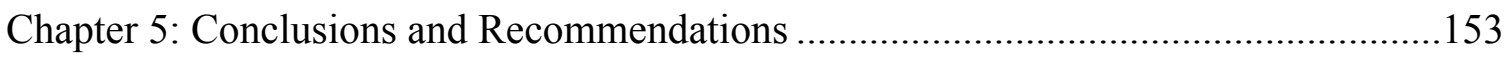

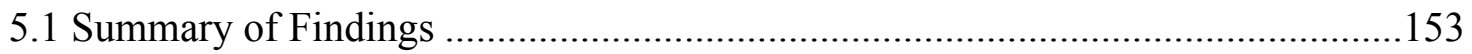

5.1.1 Finite Element Modelling of Post-Tensioned Timber Beams .........................154

5.1.2 Timber Adhesive Performance after Fire Damage .........................................156

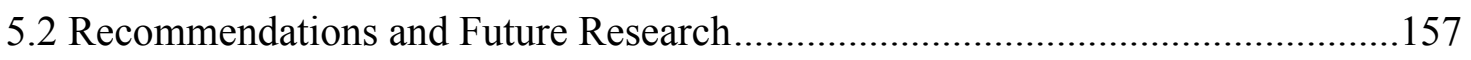

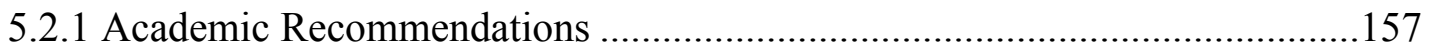

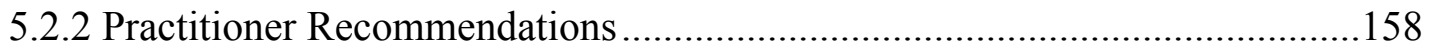




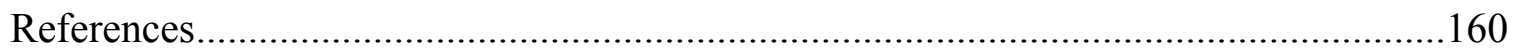

Appendix A - Developing Fire Safety Engineering as a Practice in Canada ..................172

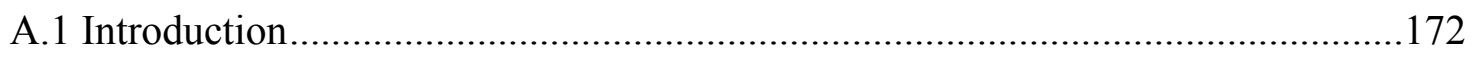

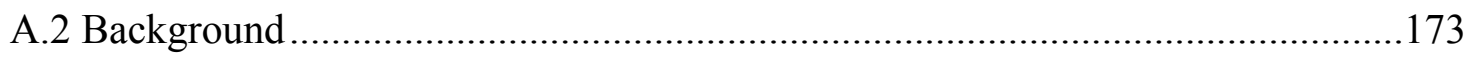

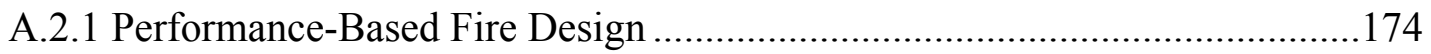

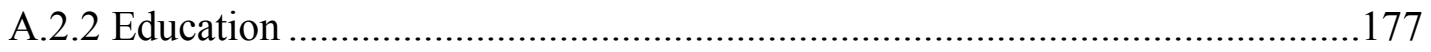

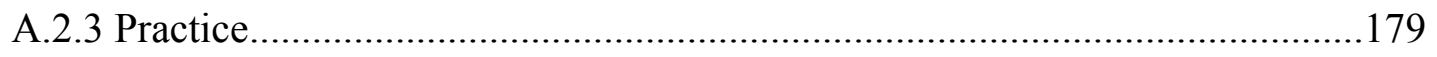

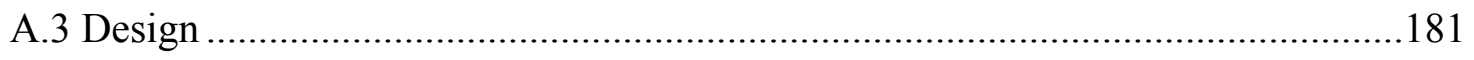

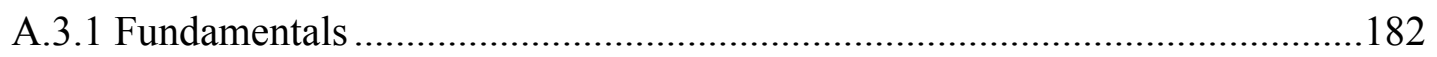

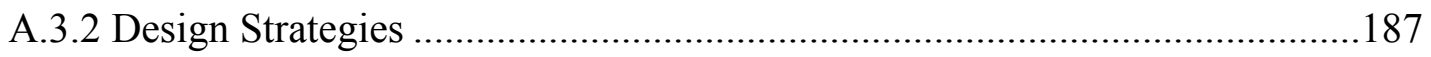

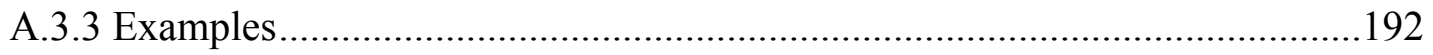

A.4 The Future of Fire Safety Engineering in Canada .............................................201

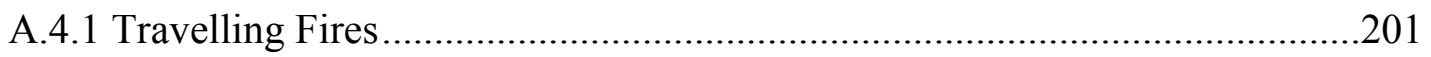

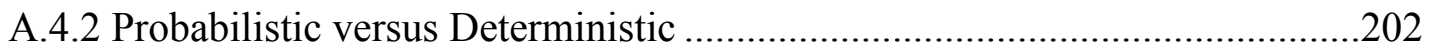

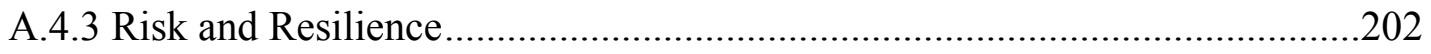

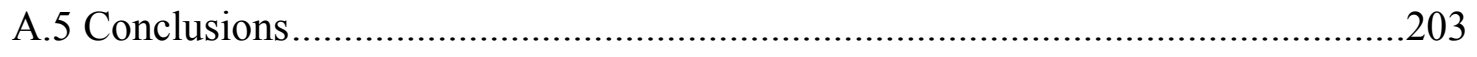

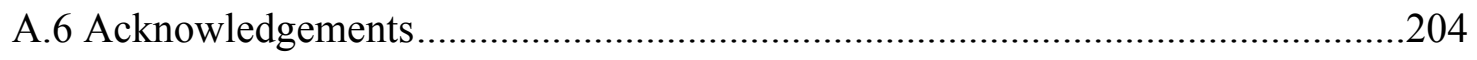

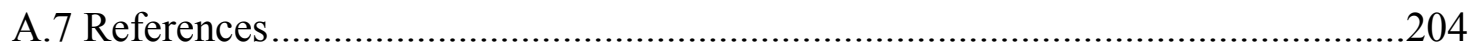


Appendix B - Finite Element Modelling of Post-Tensioned Timber Beams under Fire Conditions

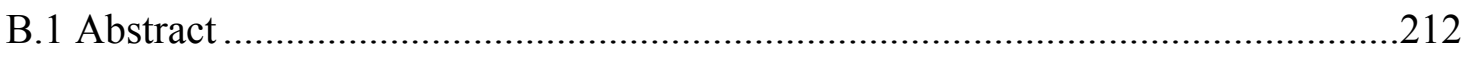

B.2 Introduction and Background....................................................................212

B.3 Model Objectives and Setup ...................................................................214

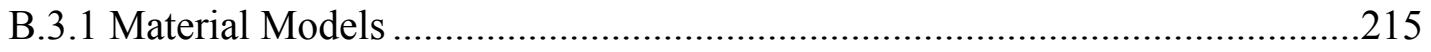

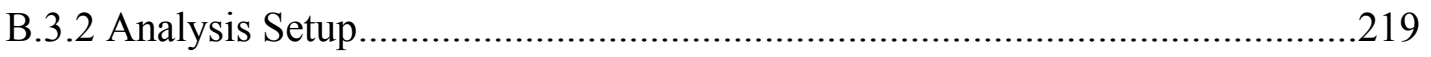

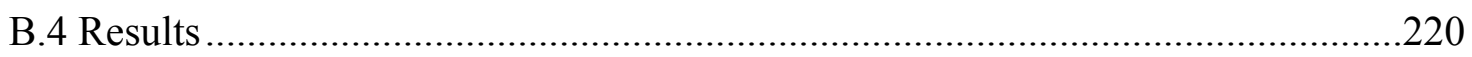

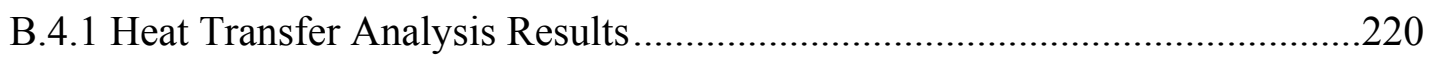

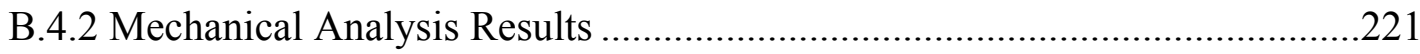

B.5 Recommendations and Conclusions ............................................................223

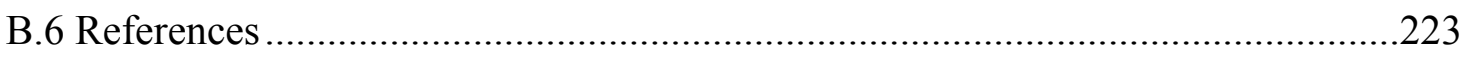

Appendix C - Performance of Adhesives in Glulam after Short Term Fire Exposure....224

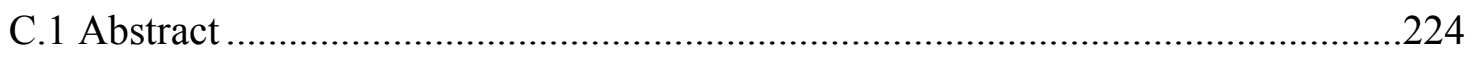

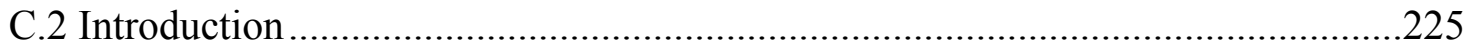

C.3 Background on Adhesive Degradation ....................................................227

C.3.1 Charring Behaviour of Engineered Timber ...........................................228

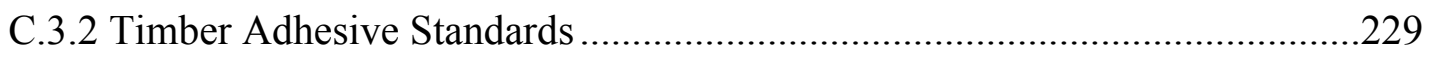

C.3.3 Research on Timber Adhesive Fire Performance .......................................2230

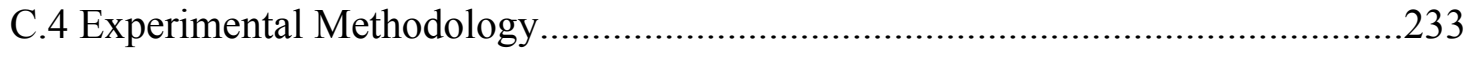


C.4.1 Phase 1: Small scale adhesive shear tests .233

C.4.2 Phase 2: large scale beam bending tests....................................................236

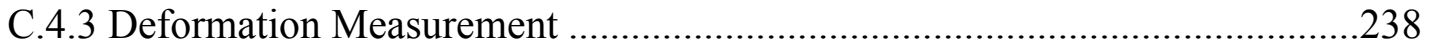

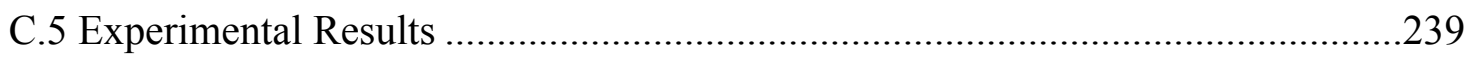

C.5.1 Phase 1: Small scale adhesive shear tests ...................................................239

C.5.2 Phase 2: Large scale beam bending tests ....................................................244

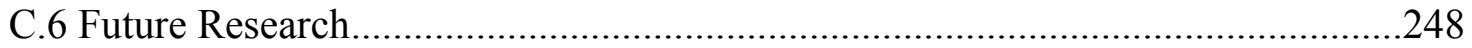

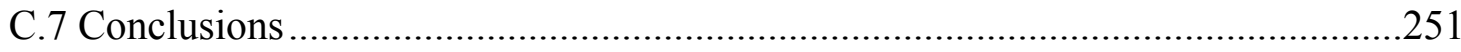

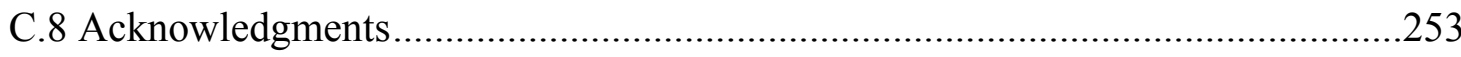

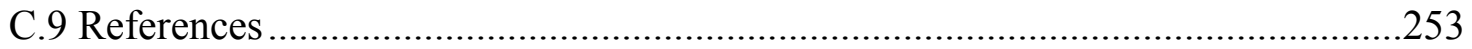

Appendix D - Adhesive Testing Data and Zero Strength Layer Calculations ................256

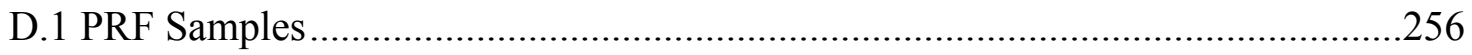

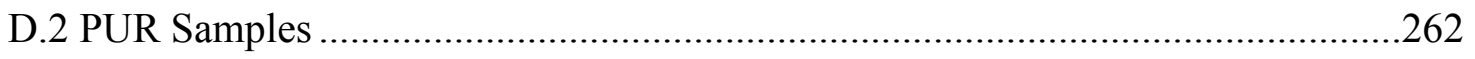




\section{List of Tables}

Table 2.1: Heavy Timber minimum dimension requirements summarized from Ontario

Building Code. .11

Table 2.2: Resulting material parameters from Van Beerschoten experimental testing of

LVL (Van Beerschoten 2013) 30

Table 2.3: Summary of tests completed by Spellman (2012)..........................................35

Table 2.4: Summary of tests completed by Costello (2013) ……......................................37

Table 2.5: Summary of failure loads and corresponding mid-span deflections from

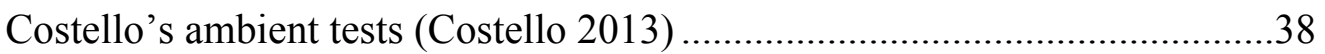

Table 2.6: List of PT timber buildings in New Zealand ..................................................40

Table 3.1: Outline of tests to be modelled, with more detailed descriptions of the past

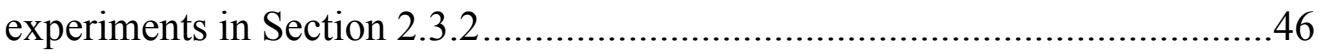

Table 3.2: Comparison of deflection results for various support restraint and load conditions .55

Table 3.3: Comparison between different material orientation definitions for the same mechanical properties

Table 3.4: Input parameters for engineering constants orthotropic elasticity .63

Table 3.5: Summary of a preliminary comparison between different model options for nonlinear material modelling of wood .65

Table 3.6: Strength properties used in Hill's Potential Function plasticity model .67

Table 3.7: Concrete Damaged Plasticity material model input parameters .71

Table 3.8: Input parameters for engineering constants orthotropic elasticity as a function of temperature .105 
Table 3.9: Strength values used in each of the material directions for calculation of the Hill's Potential Function ratios 109

Table 3.10: Strength ratios used in Hill's Potential Function for plasticity .109

Table 3.11: Elastic modulus and tension and compression strength values as a function of temperature for the CDP model

Table 3.12: Comparison between numerical model and experimental failure loads and deflections. 122

Table 3.13: Comparison between numerical model and experimental char depths .123

Table 3.14: Comparison between numerical model and experimental failure times .124

Table 4.1: Summary of Previous Experiments 127

Table 4.2: Summary of the number of samples tested for each adhesive type, incident heat flux exposure and heating duration 131

Table B.1: Input parameters for engineering constants orthotropic elasticity as a function of temperature. 216

Table B.2: Strength values used for calculation of the Hill's Potential Function ratios .218

Table B.3: Input parameters for strength ratios used in Hill's Potential Function for plasticity 218

Table B.4: Comparison of beam experiment ${ }^{2,3}$ and model failure times .222

Table C.1: Summary of the number of samples tested for each adhesive type, heat exposure and heating duration .235 


\section{List of Figures}

Figure 2.1: Schematic of production and grain orientation for (a) Glulam and (b) LVL..13

Figure 2.2: Standard Fire time-temperature curve (ISO 2014) ....................................14

Figure 2.3: Layers of material degradation in timber exposed to fire and heat transfer

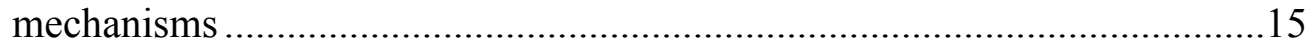

Figure 2.4: Canadian timber adhesives standards summary .......................................19

Figure 2.5: Eurocode EN 1995 Part 1-2 sample suggested effective thermal properties for wood (adapted from CEN 2004b) .......................................................25

Figure 2.6: Schematic of typical PT timber beam cross-section showing built up wood webs and flanges and high-strength steel tendons, along with section cut through beam length showing tendon anchorage .33

Figure 2.7: Overview of beam cross-sections for Spellman's tests (Spellman 2012) outlined in Table 2.3 below (all dimensions in millimeters) .35

Figure 2.8: Overview of beam cross-sections for Costello's tests (Costello 2013) outlined in Table 2.4 below (all dimensions in millimeters) .37

Figure 3.1: Sample model assembly (a) without PT tendon and (b) with PT tendon and anchorage plates (shown with view cut) .52

Figure 3.2: Various model boundary conditions: (a) line BC on beam nodes, (b) tied constraint between steel plate (master) and beam (slave), (c) surface-to-surface contact between steel plate (master) and beam (slave), (d) distributed BC on steel plate, (e) concentrated $\mathrm{BC}$ on reference point and (f) reference point coupling to steel plate..... .53 
Figure 3.3: Comparison of contour results for stress in the vertical axis (S33) on deformed shapes (magnified x5) for (a) line BC on beam nodes, (b) distributed BC with surface-to-surface contact, (c) reference point BC with surface-to-surface contact and (d) distributed BC with tied constraint. .56

Figure 3.4: PT system model: (a) tied constraint between steel anchorage plate (master) and beam end (slave) and (b) tied constraint between steel anchorage plate (master) and steel tendon end node (slave)..... .57

Figure 3.5: Load application in model by steel loading plate showing (a) steel plate (master) tied to beam top surface (slave), (b) pressure load applied to top loading plate surface, (c) reference point coupled to the loading plate surface and (d) displacement imposed on reference point .58

Figure 3.6: Section of beam assembly showing where bolt load is applied to tendon central partition (highlighted segment of wire), shortening the partition until the specified force is achieved while placing the rest of the tendon in tension ...59

Figure 3.7: Load versus deflection plot for simulations of beams using various linear elastic material models, with displacement controlled loading, compared to experimental results 60

Figure 3.8: Bilinear symmetrical constitutive model used in ambient numerical models due to material definition constraints .68

Figure 3.9: Representation of required parameters for CDP showing (a) the hydrostatic axis and deviatoric stress plane in the principal stress space and (b) the DruckerPrager function in a meridional plane as defined by the dilation angle and eccentricity .70 
Figure 3.10: Constitutive model implemented for the strength in CDP material definition

Figure 3.11: Sample crack development in quick XFEM trial (a) from maximum principal stress (b) .73

Figure 3.12: Plane stress transformations for pure shear condition................................ 74

Figure 3.13: Resulting crack from XFEM analysis with deformations magnified 5x.......76

Figure 3.14: Element type comparison for model C1 with a purely elastic analysis ........78

Figure 3.15: Longitudinal mesh refinement study of purely elastic analysis for model C1, with the full analysis (above) and the end of the analysis magnified to show variance (below)

Figure 3.16: Longitudinal mesh refinement study for model C1 with Hill's Potential Function for plasticity implemented .80

Figure 3.17: Comparison between $\mathrm{C} 1$ numerical model results and experimental data comparing multiple material models, where CDP gave identical results to Isotropic Elasticity and XFEM gave identical results to Orthotropic Elasticity (Costello 2013) .81

Figure 3.18: Numerical model results for $\mathrm{C} 1$ showing stresses perpendicular-to-grain in the vertical direction, with crushing around the support and excessive tensile stresses at the top flange to web connections .83

Figure 3.19: Comparison between numerical results and experimental data for $\mathrm{C} 2$ for models including only orthotropic elasticity and with Hill's Potential Function plasticity (Costello 2013) .84 
Figure 3.20: Comparison between numerical results and experimental data for $\mathrm{C} 2$ including the attempted failure simulation (Costello 2013) .85

Figure 3.21: C2 Numerical model results showing half of beam shown - as stresses were symmetrical - with (a) longitudinal stresses $(\mathrm{Pa})$ and (b) shear stresses $(\mathrm{Pa}) 86$

Figure 3.22: C2 Numerical model results showing (a) vertical deflection (m) - half of beam shown as deflections were majorly symmetrical, and (b) lateral deformation (m) .86

Figure 3.23: Comparison between numerical results and experimental data for $\mathrm{C} 3$ for models including only orthotropic elasticity and with Hill's Potential Function plasticity implemented (Costello 2013) .88

Figure 3.24: Comparison between numerical results and experimental data for $\mathrm{C} 4$ for models including only orthotropic elasticity and with Hill's Potential Function plasticity implemented; deflections normalized after post-tensioning (Costello 2013) .89

Figure 3.25: Comparison between numerical results and experimental data for $\mathrm{C} 4$ including the attempted failure simulation; deflections normalized after post-tensioning (Costello 2013) 90

Figure 3.26: Heat exposure applied on surfaces shown in red on 2D and 3D analyses, where the end of the beam outside the furnace and the top surface was left unexposed

Figure 3.27: Radiation applied on cavity surfaces shown in red on 2D and 3D analyses.93 
Figure 3.28: Combined element size mesh with smaller element dimensions through the thickness of the exposed flange and webs to capture more detailed heat transfer

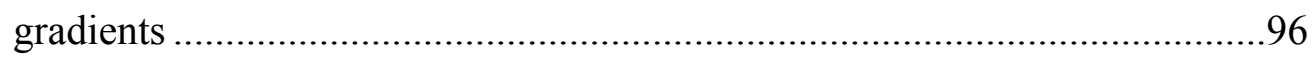

Figure 3.29: Mesh refinement study for beam cross-section thermal analysis (outside node results are shown magnified to the first 300 seconds of the analysis as mesh sizes mostly converged after this time) .96

Figure 3.30: Numerical model heat transfer results for interior web surface temperature versus experimental data for C5 (Costello 2013) .98

Figure 3.31: Comparison of C5 char depth image - modified to fix camera angle distortion from (Costello 2013) - and numerical model heat transfer at $300^{\circ} \mathrm{C}$ maximum, with an overlay (left) and a trace (middle) of the numerical results (right) ...99

Figure 3.32: Partitions for irregular thermocouple placement where the outermost partition is $6 \mathrm{~mm}$ away from the outer surface (with one $6 \mathrm{~mm}$ element), the innermost partition is $13 \mathrm{~mm}$ in from the interior surface (with three $4.33 \mathrm{~mm}$ elements), and the middle partition having $5.5 \mathrm{~mm}$ elements to ensure a node is located every $11 \mathrm{~mm}$, corresponding to thermocouple placement

Figure 3.33: Web temperature distribution comparison for S6 experimental results versus numerical model (Spellman 2012) .101

Figure 3.34: Bottom flange temperature distribution comparison for S6 experimental results versus numerical model (Spellman 2012) .101

Figure 3.35: Web temperature distribution comparison for S7 experimental results versus numerical model (Spellman 2013) .102 
Figure 3.36: Bottom flange temperature distribution comparison for S7 experimental results versus numerical model (Spellman 2013) .103

Figure 3.37: Results of Hill's plasticity model with reduced stiffness elastic orthotropic behaviour compared to experimental value for C5 (Costello 2013), with total and normalized deflections for the fire duration under constant loading (i.e. normalized does not include prior deflections from pre-fire loading and posttensioning)

Figure 3.38: Shear stresses versus strength (reduced for average element temperature according to Eurocode 5 Part 1-2 Annex B) in web elements for a critical shearregion cross-section (CEN 2004b) .108

Figure 3.39: Element sizes for mesh sensitivity study on model C5 where each web and flange element is $45 \mathrm{~mm}$ thick; dimensions for Beam 1 given in Figure 2.8

Figure 3.40: Mesh sensitivity study for model C5 with three sizes of mesh elements....112

Figure 3.41: Vertical displacement versus time into heat exposure for C5 is shown compared to the numerical model results from the Hill's Potential Function and the CDP models (Costello 2013)

Figure 3.42: Comparison of failure modes in numerical model (above) and experimental beam (below) where a shear region failure between the support outside the furnace and the load application point can be seen (Costello 2013).... .114

Figure 3.43: Stress due to thermal expansion of the tendons versus the post-tensioning stress in the C5 numerical model .115 
Figure 3.44: Vertical displacement versus time into heat exposure for S6 is shown compared to the numerical model results from the Hill's Potential Function model (Spellman 2012) 116

Figure 3.45: Comparison between model failure mode (left) and beam after furnace test (right) where slight buckling at the furnace edge interface can be seen (Spellman 2012) .117

Figure 3.46: Comparison of S6 numerical model results to experimental data for the average tendon force versus time into heat exposure (Spellman 2012).......118

Figure 3.47: Stress due to thermal expansion of the tendons versus the post-tensioning stress in the S6 numerical model .118

Figure 3.48: Vertical displacement versus time into heat exposure for S7 is shown compared to the numerical model results from the Hill's Potential Function model (Spellman 2012)

Figure 3.49: Comparison of the S7 numerical model results to experimental data for the average tendon force versus time into heat exposure (Spellman 2012)......120

Figure 3.50: Stress due to thermal expansion of the tendons versus the post-tensioning stress in the S7 numerical model

Figure 4.1: Glulam box-beam four-point loading tests comparative load versus displacement data (Quiquero et al. 2016). .128

Figure 4.2: Cone Calorimeter apparatus showing (a) the hood used to collect test emissions for calorimetry, (b) the apparatus display, (c) a specimen during heating with the shield deployed and (d) the conical heating element during heating .....132 
Figure 4.3: Schematic for construction of steel shear test apparatus showing hatched wooden block within apparatus (all dimensions in millimeters). .135

Figure 4.4: Shear plane shown on unheated and heated samples (left) and shear apparatus test setup (right) .135

Figure 4.5: Sample heat release rate curves of wood samples measured by cone calorimeter; one sample of each adhesive type for both $30 \mathrm{~kW} / \mathrm{m}^{2}$ and $50 \mathrm{~kW} / \mathrm{m}^{2}$ incident heat flux exposures for 10 minute duration tests are shown .138

Figure 4.6: Total char depths measured on samples versus the heating duration .139

Figure 4.7: Charring rate averaged over the entire heating duration for each sample versus its heating duration

Figure 4.8: Samples after burning exposed at $50 \mathrm{~kW} / \mathrm{m}^{2}$; on the left a PRF sample exposed for 3 minutes and on the right a PUR sample exposed for 6 minutes

Figure 4.9: Failure stress of each PUR sample tested compared to heating duration of that sample with linear trend lines depicted for each heating exposure

Figure 4.10: Failure stress of each PRF sample tested compared to heating duration of that sample with linear trend lines depicted for each heating exposure .142

Figure 4.11: Comparison of remaining shear capacity versus area for all samples .143

Figure 4.12: Remaining shear capacity of samples versus different predictions of actual remaining shear area at full adhesive strength, compared with the measured char depths and the code predicted sacrificial char method. 146

Figure 4.13: Schematic of cross-section through shear plane in specimen showing the area used to calculate the full-strength zone and thus the additional zero-strength layer .147 
Figure 4.14: Load versus slip behaviour for several samples heated for different durations under a $50 \mathrm{~kW} / \mathrm{m}^{2}$ incident heat flux, with PUR adhesive.

Figure 4.15: Load versus slip behaviour for several samples heated for different durations under a $50 \mathrm{~kW} / \mathrm{m}^{2}$ incident heat flux, with PRF adhesive .149

Figure 4.16: Slip at failure versus failure load for each specimen for which DIC was able to be completed, with linear trend lines included for each heating duration 150

Figure A.1: In Toronto alone, there are now over 25 complex and highly optimized buildings that are 50 stories or higher. Nearly 15 are under construction and nearly 30 are approved and/or proposed. Additionally, to accommodate the urbanization, significant and complex infrastructure is proposed. Each engineering project may have the potential for application of advanced FSE to facilitate safe best practice benefits seen globally. (Author's photo). 173

Figure A.2: The Greater London Authority Building was one of the first projects approved in London using modern FSE concepts and strategies which allowed the unique building to be executed (O’Meagher and Ferguson 2003). (Author's photo). 195

Figure A.3: The Shard high-rise building in London, England used innovative fire strategies to incorporate the functionality and spatial needs of the building (Kitching 2012). (Author's photo).

Figure B.1: Schematic of typical PT timber beam cross-section showing built up wood webs and flanges and high-strength steel tendons, along with section cut through beam length showing tendon anchorage with a steel plate at the end 
Figure B.2: Eurocode 5 Part 1-2 Annex B suggested reduction factors for elastic modulus (left) and strengths (right) ${ }^{4}$ 215

Figure B.3: Comparison between experimental ${ }^{2}$ and numerical thermal results through the web thickness for Spellman's Beam A (left) and Beam C (right) .221

Figure B.4: Comparison between experimental ${ }^{2,3}$ and numerical deflection results ......222

Figure B.5: Failure region in left side shear zone in simulation results and experiment (modified from ${ }^{3}$ ) .223

Figure C.1: Canadian timber adhesives standards summary (see Quiquero and Gales, 2017) .230

Figure C.2: Shear plane shown on unheated and heated samples (left) and shear apparatus test setup (right). .235

Figure C.3: Test setup of the heating portion of the experiment for the moment region (left) and the shear region (right) .236

Figure C.4: Mechanical loading of simply supported beam with four point loads. ........238

Figure C.5: Total char depths measured on samples versus the heating duration ...........240

Figure C.6: Comparison of remaining shear capacity versus area for all samples.........241

Figure C.7: Remaining shear capacity of samples versus different predictions of actual remaining shear area at full adhesive strength, compared with the measured char depths and the standard predicted sacrificial char method. .242

Figure C.8: Load versus slip behaviour for heated for different durations under a $50 \mathrm{~kW} / \mathrm{m}^{2}$ incident heat flux 244

Figure C.9: Failure load of all beams damaged in the moment region, shear region, as well as the control beams .246 
Figure C.10: Load versus downward displacement beams damaged in the moment region

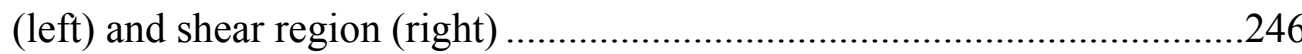




\section{Statement of Co-Authorship and Scholarly Outputs}

This thesis and the work described within have been completed by Hailey Quiquero under the supervision of Dr. John Gales at Carleton University, and in collaboration with Dr. Anthony Abu at the University of Canterbury. While not identical to the published works, many chapters have been formed into conference or journal papers as follows, with each publication being primarily written by Hailey Quiquero.

Chapter 1: The first chapter provides a general introduction to the thesis collectively and presents the motivation, objective and outline of the work. The text in Section 1.1.2 refers to a journal paper, written primarily by the author but also in collaboration with Matthew Smith of Entuitive Consulting Engineers in Toronto, Ontario and supervisor John Gales, which is included in Appendix A.

Quiquero, H., Smith, M., and Gales, J. (2018), "Developing Fire Safety Engineering as a Practice in Canada," Canadian Journal of Civil Engineering, NRC

Press, Ottawa, Canada. doi: 10.1139/cjce-2016-0552

Chapter 2: This chapter is a combined literature review for all components of this project and gives background information on research conducted and related existing knowledge and standards for massive timber construction and engineered timber products. It focuses specifically on structural fire engineering in Canada and with timber construction, engineered timber adhesives, numerical modelling of timber in fire conditions, and the post-tensioned (PT) timber structural system.

Chapter 3: The work described in this chapter was completed in New Zealand in collaboration with Dr. Anthony Abu of the University of Canterbury. A conference paper 
was presented based on this chapter (cited below and included in Appendix B) and it is intended to be submitted for publication as a two-part journal paper.

Quiquero, H., Gales, J., Abu, A., Moss, P. (2018), “Finite Element Modelling of Post-Tensioned Timber Beams under Fire Conditions," SFPE 12th International Conference: Fire Engineering Solutions for the Built Environment, Hawaii, United States, April 26-28, 2018.

Chapter 4: This chapter describes continued work based on research submitted for an undergraduate project. The work that provided the basis for this research was presented at two conferences:

Quiquero, H., Gales, J., Hadjisophocleous, G. (2016), “Behaviour of Char Layer in Fire-Damaged Box Section Timber Beams," 14th International Conference and Exhibition on Fire Science and Engineering (Interflam 2016), Egham, United Kingdom, July 4-6, 2016, pp. 1063-1074.

Quiquero, H., Gales, J. (2016), “Behaviour of Fire-Damaged Engineered Timber Beams," CSCE 2016 5th International Structural Specialty Conference, London, Canada. Canadian Society for Civil Engineering, Canada, June 1-3, 2016.

The subsequent experiments that were completed for the master's degree are described in this chapter. A conference paper was published based on the information in this chapter:

Quiquero, H., Gales, J. (2017), “Comparing Timber Adhesive Shear Strength Properties after Fire Damage," 15th International Conference on Fire and Materials 2017, San Francisco, United States, February 6-8, 2017. 
Additionally, portions of the chapter were submitted in a manuscript for a journal paper (which is included in Appendix C):

(Submitted) Quiquero, H., Chorlton, B., Gales, J. (2018), "Performance of Adhesives in Glulam after Short Term Fire Exposure," International Journal of High-Rise Buildings, Council on Tall Building and Urban Habitat, Korea.

Chapter 5: While each chapter details the conclusions from each component of research conducted, this chapter summarizes the conclusions drawn and recommendations made from all of the research completed. 


\section{Chapter 1: Introduction}

\subsection{Structural Fire Engineering}

For centuries, wherever infrastructure is built, there has been societal concerns of fire protection in structures. In 1865, a prominent British architect Thomas Hayter Lewis gave a presentation for the members of the Royal Institute of British Architects about fire proofing in construction, alluding to the challenges of structural fire engineering solutions (Lewis 1865). Even at this time, over a century ago, he mentions the struggles of approval authorities at the time allowing structures which were not conceived of when the building acts were framed. This is analogous to current difficulties in the approvals of structures which are outside legislative regulations by authorities having jurisdiction (AHJ). Infamous conflagrations such as the Great Fire of London in 1666 or the Great Chicago Fire in 1871, along with hundreds of other city and individual building fire events, have inspired many historic fire testing and regulations that remain in place today. These tests from over a hundred years ago are also the reason for these challenges in constructing structures outside the building code restrictions.

Structural Fire Engineering (SFE) is the field of using engineering judgement and analysis to design safe building solutions for critical fire scenarios. It is a relatively nascent field, especially when compared to other engineering disciplines such as Mechanical Engineering or within the overarching field of Civil Engineering. Although the notions of SFE have been considered for centuries, highly conservative codes have been the solution until recent years, during which technological developments have allowed for more sophisticated predictions and analyses. There remains a dearth of research into the basis of 
material data and behaviour that is required to use such intelligent and complex technologies, along with how the available data may be implemented into the software packages. This is especially true for timber and has thus sparked the need for this research.

\subsubsection{Performance-Based Fire Design}

SFE differs from code-consultancy based practices which use a prescriptive approach to fire safety. Building code clauses have been developed in order to simplify the approval of buildings for fire safety in the form of exit requirements, detection systems, evacuation plans, etc. For example, Part 3 in Division B of the National Building Code of Canada outlines the Acceptable Solutions for Fire Protection, Occupant Safety and Accessibility. This code places restrictions on floor areas, stories, exit distances and widths, and fire resistance ratings of various assemblies. The strict requirements of these prescriptive clauses may, at times, inhibit a project from reaching its true potential. This is specifically true for large, modern commercial buildings in which the architectural vision does not coincide with these restrictions. This is where performance-based fire design (PBFD) becomes an ideal alternative.

The late Margaret Law was one of the pioneers of PBFD in the United Kingdom in the late $20^{\text {th }}$ century. In a publication on the subject from 1995, she stated that "The desire of regulators to have simple rules and tests for administrative convenience contrasts with the need of designers to have maximum flexibility in order to arrive at optimum solutions. The magic numbers embodied in regulations are accepted without question, while any engineering solution is subject to a disproportionately high standard of proof." This declaration remains currently true as it always has in structural fire engineering. The building code clauses previously discussed are based on empirical observations from fires 
and experiments done decades ago, for buildings that don't necessarily correspond to the structures of today. The level of fire safety or risk is not quantified in any way, and yet these solutions are acceptable for all newly proposed structures. However, an engineering analysis using numerical modelling and comprehensive calculations is viewed under high scrutiny and is met by authorities having jurisdiction unequipped to approve of such a solution.

PBFD can be very useful in many projects where the building code fire regulations prove too restrictive for the architectural vision to be accomplished. Additionally, it may be advantageous in scenarios where the code is proven to be highly conservative, and costs of fire protection measures may be reduced. In highly complex buildings, at times, the building code provisions may also be found to be under-conservative compared to fire simulations or occupant egress modelling. PBFD is a delicate balance of providing buildings with sufficiently low risk to occupants and property, while maintaining an efficient and cost-effective solution for clients.

\subsubsection{Structural Fire Engineering in Canada}

In Canada, PBFD in SFE is not a very prevalent practice. Code consultants are often hired for projects to ensure the structure will meet fire code requirements, and that is the only way fire safety is typically considered. However, the National Building Code of Canada (NBCC) is set up so that practitioners may comply with the code with an "Alternative Solution" to the prescriptive code clauses, as set out in Division A, Part 1: "1.2.1.1.(b) Compliance achieved using alternative solutions that will achieve at least the minimum level of performance required by Division $B$ in the areas defined by the objectives and functional statements attributed to the applicable acceptable solutions" (NRC 2015). 
There is information in Division A, Parts 2 and 3 of the NBCC about the objectives and functional statements of the provisions for the Acceptable Solutions in Division B. Thus, structural fire engineers are able to do an analysis and design in order to provide a solution which meets the objectives of the code. This slightly differs to how SFE is done abroad as there are no performance metrics given in the objectives section; this is known as objectivebased design (OBD) rather than PBFD. The solution must simply meet the objectives at the same level of performance which would be achieved by the acceptable solutions given in the requirements of Division B. Thus, substantial additional work is required to demonstrate the performance of the proposed Alternative Solution compared to the code required design.

An extensive review of the state-of-the-art in SFE and PBFD in Canada was published by the author (along with collaborators) covering a wide range of projects and best-practices for practitioners (see Appendix A).

\subsubsection{Building and Innovating with Timber}

Recent years have seen the development of innovative timber products and construction systems as society pushes for sustainable and aesthetically pleasing structures that connect with nature. Building codes such as the NBCC are being updated to allow for taller combustible construction, with up to 12 -storey encapsulated massive timber construction (EMTC) being endorsed by the Standing Committee on Fire Protection of the Canadian Commission on Building and Fire Codes (CCBFC) for the next update in 2020 (NRC 2018). Additionally, local municipalities are pushing the envelope of code approvals with high-rise timber buildings, many encapsulated in non-combustible materials. However, there is significant research to suggest that we are still unaware of the unique mechanisms 
in the ways timber performs and fails, especially in fire conditions, such as identified by Jeanneret et al (2017). It is imperative to investigate further into the detailed performance of timber under fire exposure and specifically into the behaviour and resilience of engineered timber products and adhesives. More background on timber structures in fire and related research findings are presented in the following chapter.

\subsection{Motivation and Research Objectives}

It is clear that the field of SFE is gradually developing in North America, and Canada should expect to see more alternative solutions and objective-based designs on projects in the coming years. An increased environmental conscientiousness in society and the abundance of timber in Canada has inevitably lead to the desire for more timber construction, and the proposed NBCC 2020 amendments to allow for up to 12-storey EMTC. In order to increase the opportunity for timber products to be used in large-scale commercial construction, novel building systems such as PT timber are required. Such complex building systems likely require detailed design solutions for approval. It is often desired to have timber structural systems exposed in buildings for aesthetic purposes, which places additional complications on the analysis.

In order to propose design tools which benefit practitioners for such novel systems and materials, it is of the utmost importance to have a deep understanding of the material's performance. This is specifically true of a combustible material in fire conditions. The natural origin of wood means that it certainly has variability in its characteristics, although this is lessened to some degree with engineered timber products. However, there are also new challenges introduced in these products, including the adhesive performance. In complex systems which have thinner sections of wood in the cross-section and rely heavily 
on the behaviour of those timber products, an intimate knowledge of how they will perform is required.

The ultimate goal of this research study was to facilitate guidance for the use of PT timber as a structural system in Canada. The foremost objective in order to achieve this was to study the performance of the PT timber system. A study was completed abroad in collaboration with researchers from the University of Canterbury in New Zealand who have completed the only previous furnace fire experiments on PT timber beams. The results of those experiments were discussed and a numerical model for the performance of PT timber beams in fire conditions was developed and validated based on those tests. The goal for the model was to build it based upon the knowledge gathered from all previous experiments on Glulam and PT timber in an existing FEM platform, so eventually it may be used for a priori modelling for future large-scale tests. A model was developed in Abaqus in order to investigate the feasibility of available FEM software packages to model PT timber in fire conditions, and to eventually inform a tool for practitioners to design and implement the structural system. Additionally, it was desired to increase the knowledge base and data available on the performance of the engineered timber product, Glulam, which is commonly used in large-scale commercial construction in Canada. The performance of the adhesive after fire damage was examined after previous research suggested further investigation was required.

\subsection{Research Scope and Thesis Outline}

Research on the behaviour of massive timber structures in fire encompasses an expansive list of topics. There remain large gaps in the research and available data for timber during fire conditions and fire-damaged timber in all aspects including ignition, flame spread, 
smouldering, charring models, non-standard fire exposures, delamination, cooling phases, and self-extinguishment. Due to the extensive range of topics and various wood species and products, it was necessary to limit the scope of this research project. It is additionally important to note that wood properties may vary from species to species, or within species grown in different geographical regions, so the parameters adopted and suggested herein may not be entirely accurate for all species of softwood engineered timber products.

\subsubsection{Chapter 2 - Background and Literature Review}

This chapter attempts to summarize all the background information and previous research required to understand and appreciate the work of this research project. Chapter 2 covers background knowledge on timber products, adhesives and structures in fire including summarizing past experiments and standards. Past efforts in the numerical modelling of timber in ambient and fire conditions are summarized. Finally, an introduction to PT timber is given with an outline of the system, past experiments and existing PT timber buildings.

\subsubsection{Chapter 3 - Finite Element Modelling of Post-Tensioned Timber Beams}

Chapter three discusses the development and validation of the numerical model and how the results compare to the experiments done at the University of Canterbury. The model development was limited to the use of existing material models within Abaqus in order to evaluate the existing abilities of FEM platforms and to achieve model results within the project collaboration (thus a material defined by a user-defined subroutine was considered outside the scope of this project).

This chapter describes in great detail the development and implementation of the model in Abaqus, including the selection process of the FEM software package and material model 
decisions. The numerical model results are then compared to previous test data made available through the collaboration with the University of Canterbury.

\subsubsection{Chapter 4 - Timber Adhesive Performance after Fire Damage}

Chapter four discusses the experimental component of the project, where experiments were done on Glulam samples to get a detailed understanding of the material fire performance and research gaps. It was decided to limit the adhesive testing to small-scale samples in order to study in detail the behaviour of the adhesive and perform numerous tests, varying parameters and including duplicates for repeatability. The mechanical testing was done after fire exposure to be in line with previous experiments by the author (Quiquero and Gales 2016, Quiquero et al. 2016) in which specimens were tested after cooling to allow for detailed investigation of the load-deflection behaviour. Thus, Digital Image Correlation (DIC) was utilized to capture in detail the failures of the small adhesive specimens postheating.

In this chapter, the results of the experiments are analyzed and discussed, and the implications of the findings are detailed. An increased zero-strength layer beyond the char front is discussed due to adhesive degradation but is limited by the small-scale of the samples tested.

\subsubsection{Chapter 5 - Conclusions and Recommendations}

The final chapter gives conclusions on the results of the study, on how the two phases of the project affect the use of the PT timber building system in Canada, and how the results may be used by both practitioners and researchers to advance the knowledge and implementation of complex timber structures. 


\subsubsection{Appendices}

Following the five main chapters, there are four appendices. Appendix A, Appendix B, and Appendix C include works published or submitted for publication throughout the duration of the degree, and Appendix D includes all of the test data and calculations from the adhesive testing and zero-strength layer calculations. 


\section{Chapter 2: Background and Literature Review}

\subsection{Timber Structures in Fire}

Timber is a material with numerous construction benefits including being a lightweight material which may be renewable. It is also aesthetically pleasing, adding to the occupant experience in a building. Wood can be viewed as highly sustainable due to its low carbon footprint and is an abundant resource in Canada. There is a common perception that wood buildings are inherently risky due to their combustible properties. However, massive timber elements have an intrinsic resistance to fire that can, with adequate knowledge and understanding, be designed to have an acceptable level of safety for occupants and property protection.

Massive timber differs from dimension lumber construction as the structural members used are larger in cross-sectional area, and the structural systems are akin to those of concrete and steel. This is contrary to dimension lumber which utilizes closely-spaced slender members as the structural system, generally for low- to mid-rise construction. In the Ontario Building Code (Ontario MMAH 2012), massive timber elements are defined as Heavy Timber Construction if they meet the dimensions summarized in Table 2.1, varying for different structural elements and supported assemblies, along with various requirements for floors and other assemblies.

Timber members with such large dimensions are able to resist loads under extended fire exposure due to the self-insulating properties of wood. Wood goes through pyrolysis as it is heated until it has totally converted to char, forming a protective layer which insulates against additional heat transfer to the unheated wood. Massive timber members, akin to 
logs you might burn for hours on a fire, will hold a great deal of their strength when exposed to extended durations of extreme heat.

Table 2.1: Heavy Timber minimum dimension requirements summarized from Ontario Building Code

\begin{tabular}{|l|l|l|l|}
\hline \multirow{2}{*}{$\begin{array}{c}\text { Supported } \\
\text { Assembly }\end{array}$} & \multicolumn{1}{|c|}{$\begin{array}{c}\text { Structural } \\
\text { Element }\end{array}$} & \multicolumn{2}{c|}{ Minimum Dimensions } \\
\cline { 2 - 4 } & Solid Sawn (mm) & \multicolumn{1}{c|}{ Glulam (mm) } \\
\hline Roof only & Columns & $140 \times 191$ & $130 \times 190$ \\
\cline { 2 - 4 } & $\begin{array}{l}\text { Beams, girders and } \\
\text { trusses }\end{array}$ & $89 \times 140$ & $80 \times 152$ \\
\cline { 2 - 4 } & $\begin{array}{l}\text { Arches, supported } \\
\text { on walls or } \\
\text { abutments }\end{array}$ & $89 \times 140$ & $80 \times 152$ \\
\cline { 2 - 4 } & $\begin{array}{l}\text { Arches, supported } \\
\text { on floor }\end{array}$ & $140 \times 140$ & $130 \times 152$ \\
\hline \multirow{2}{*}{$\begin{array}{l}\text { Floors, Floors and } \\
\text { Roof }\end{array}$} & Columns & $191 \times 191$ & $130 \times 228$ or \\
\cline { 2 - 4 } & $\begin{array}{l}\text { Beams, girders, } \\
\text { trusses and arches }\end{array}$ & $\begin{array}{l}140 \times 241 \text { or } \\
191 \times 191\end{array}$ \\
\hline
\end{tabular}

The charring rate of commonly used woods in various fire exposures has been studied in great detail for decades (including Schaffer 1967, White 2000) which is further discussed in Section 2.1.3. The consistent charring rate of wood is often used as a design tool for massive timber structural members in fire conditions, known as the Sacrificial Char Method. In this method, the char depth predicted to occur in the wood over the required fire resistance rating period is calculated. A sufficient depth of timber beyond the dimensions that are required to carry the expected loads that would occur during a fire is ensured. Often, this requirement is already fulfilled through the normal use load requirements which are larger than the loads expected during a fire. Thus, in the event of a fire, the extra timber may be allowed to char to protect the strength of the inner timber and not cause failure of the structural member. 


\subsubsection{Engineered Timber Products}

Massive timber has been used in construction for centuries as trees were one of the first materials used historically to build structures, and some are still standing after over a thousand years such as the Horyu-ji Temples in Japan, constructed before the $8^{\text {th }}$ century AD (ICOMOS 1993). With new technologies, timber can be transformed to be even more efficient as a structural material. With engineered timber products, element sizes and lengths can be manufactured for almost any application. Engineered timber products include portions of wood cut from softwood trees, typically in the form of laminates or veneers, combined with adhesive to make structural components with optimal properties. There are numerous types of these products, but some of the most common in Canada for large structural systems are glued-laminated timber (Glulam), laminated veneer lumber (LVL) and cross-laminated timber (CLT). The first two are primarily used for their longitudinal properties as beams or columns, while CLT optimally performs as a panel element in walls or floor slabs. The focus in this research is on Glulam and LVL.

Glulam is comprised of softwood laminates which may be as small as $20 \mathrm{~mm}$ by $40 \mathrm{~mm}$ in cross section, and are glued together with a structural adhesive. The pieces are cut so that the cross-section is oriented randomly between the tangential and radial perpendicular-tograin directions, as can be seen in Figure 2.1a. Laminates are connected end-to-end (parallel to the grain) with finger joints in order to increase the surface area for adhesive bonding. LVL has similar strength properties and abilities as Glulam, but it is comprised of softwood veneers rather than laminates. The veneers generally run the full depth of the element and are only around $3 \mathrm{~mm}$ thick. They are shaved around the circumference of the tree trunk so that the depth is always tangential to the grain and the thickness is always 
along the radial material direction, as is demonstrated in Figure 2.1b. Similarly to Glulam, the wood grain always runs parallel to the longitudinal direction of the structural element.

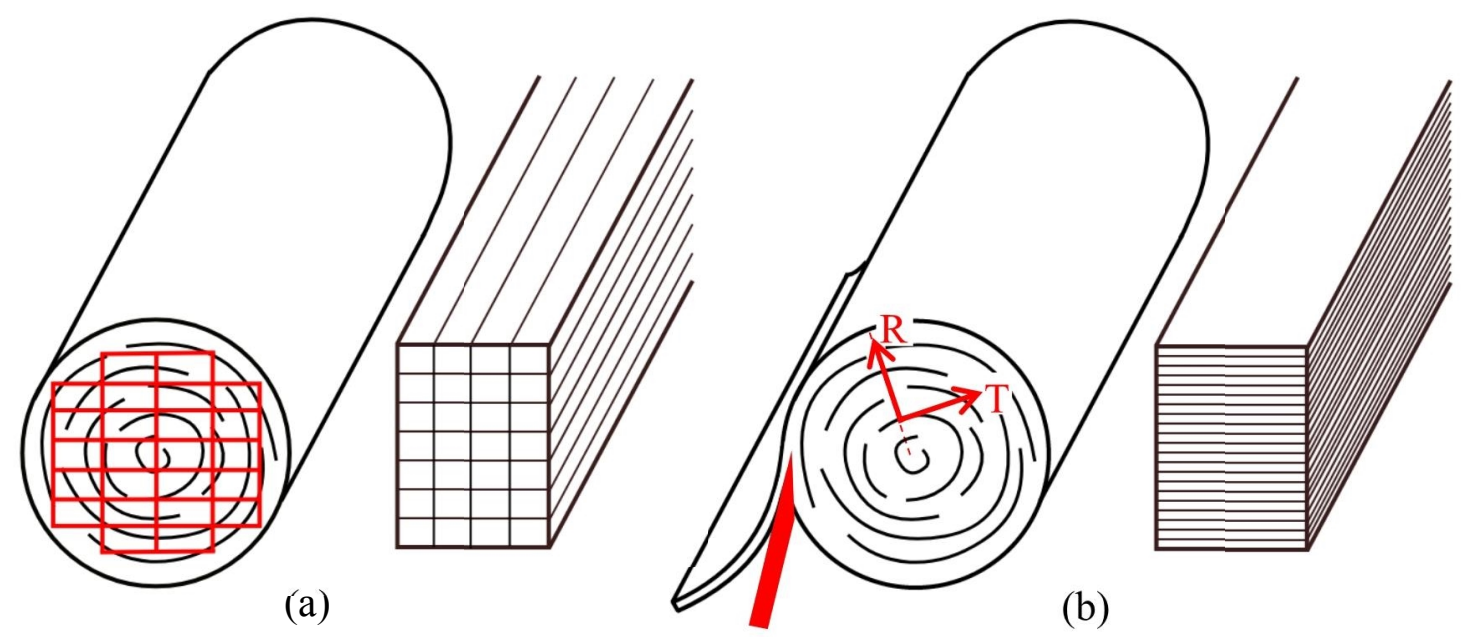

Figure 2.1: Schematic of production and grain orientation for (a) Glulam and (b) LVL The structural adhesives used in engineered timber products vary from manufacturer to manufacturer, but they must pass standardized testing to prove that it performs as well as solid-sawn lumber in high-temperature conditions (expanded on in Section 2.1.4).

\subsubsection{Standard Fire Testing}

Under the current code provisions, all construction materials and components are tested with a Standard Fire Test (ISO 2014), as is defined in ISO 834 (or equivalently, CAN/ULC S101 in Canada). This testing is typically done in a furnace with a standard timetemperature curve (shown in Figure 2.2) which was developed early in the 20th century by the ASTM, in which the temperature rises to almost $700^{\circ} \mathrm{C}$ in the first 10 minutes. By the 60 minute mark, the temperature in the fire compartment is over $925^{\circ} \mathrm{C}$, and continues to rise indefinitely. The time until which a component fails in this test is its fire resistance which is qualified as a loss of structural integrity, or too much heat or smoke penetrating the component. 


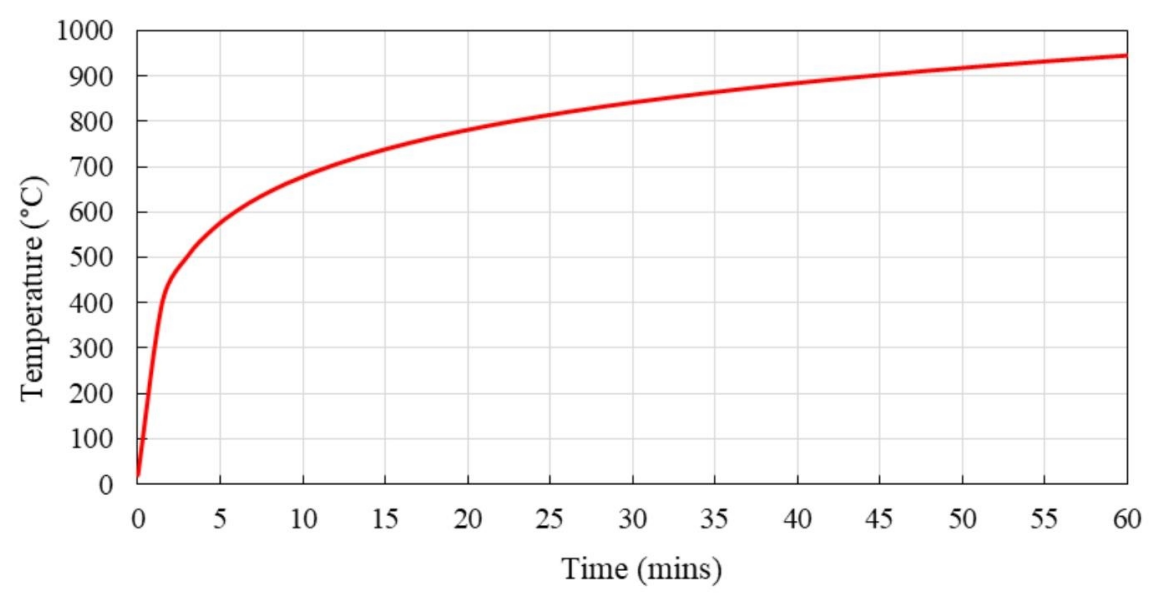

Figure 2.2: Standard Fire time-temperature curve (ISO 2014)

The test was first used in June 1917 as part of a comparative index of ratings for concrete, steel and timber columns which were placed under a service load and standard heating. In the test the isolated materials were then ranked by time of failure. The appropriateness of these tests was further limited by the difficulties of manual temperature control, especially with timber specimens which may increase the fuel load (Ingberg 1919). Additionally, the Standard Fire as defined by temperature alone is not representative of a real fire and has no physical meaning, other than providing a standardized temperature exposure (Bisby et al. 2013). Realistically, building fires can reach much higher temperatures in varying amounts of time and will enter into a decay phase after either the available fuel or oxygen is consumed.

\subsubsection{Charring Behaviour of Timber}

There has been ample research into the charring behaviour of wood over the past century, and how wood chars under standard fire conditions is generally accepted in the field. Engineered timber products are largely believed to char in much the same way that solid timber would. Under fire conditions, wood begins to undergo a process known as pyrolysis 
around $100^{\circ} \mathrm{C}$ where its chemical properties begin to change. The wood strength begins to degrade until $300^{\circ} \mathrm{C}$ which is recognised as the charring temperature of wood, at which point the pyrolysis process has completed and the wood is completely converted to char. After this point, char may continue to develop as the wood behind the char layer is heated, and the exposed char continues to oxidize until it begins to crack and flake off or is fully consumed. The phases and heat transfer which occur in this process are shown in Figure 2.3 .

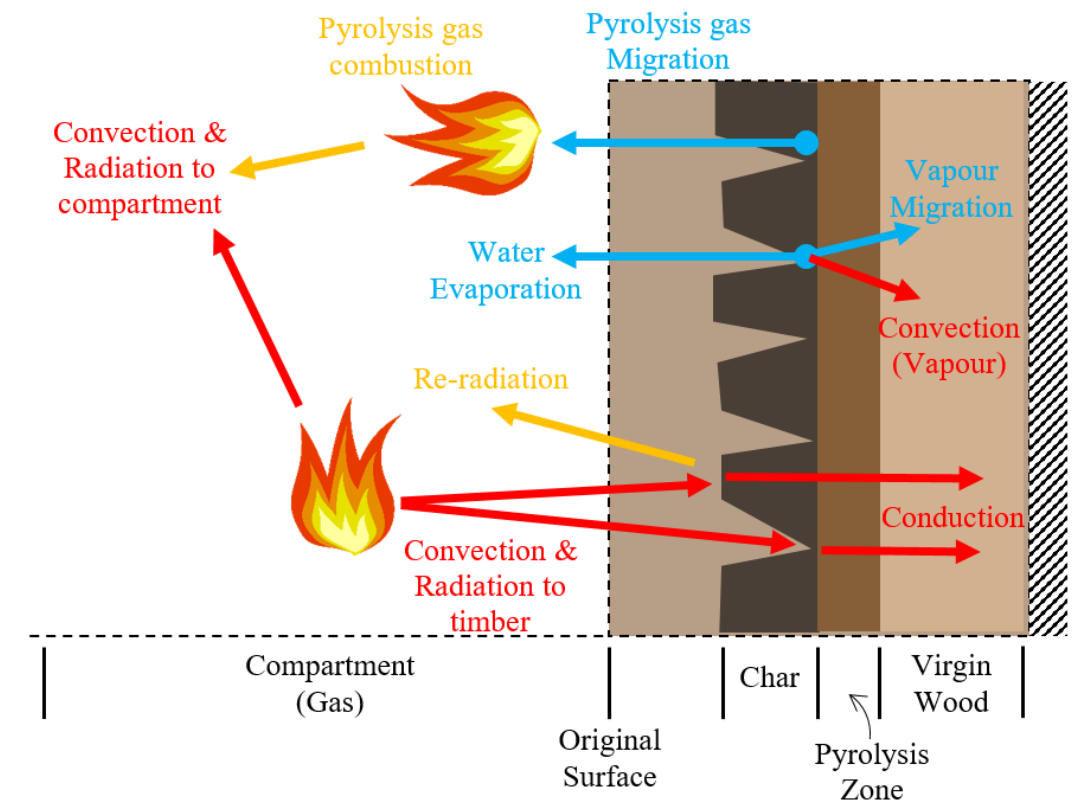

Figure 2.3: Layers of material degradation in timber exposed to fire and heat transfer mechanisms

Although the charring rate is realistically a transient property depending on the degree of char that has already formed and the amount of heat the wood is exposed to, standardized constant charring rates have been developed for building code and guideline applications. For example, a commonly recommended value for the notional standard rate of charring of engineered softwood timber such as Spruce-Pine-Fir (SPF), including Glulam and LVL, is $0.7 \mathrm{~mm} / \mathrm{min}$ (see section 3.4 in CEN 2004a). This rate is meant to encompass the initial 
rapid char phase while fresh wood builds up an insulating layer of char which then slows the charring rate to a much lower value. Additionally, the nominal rate is increased from the base one-dimensional charring rate of $0.65 \mathrm{~mm} / \mathrm{min}$ to account for corner rounding effects. However, it has also been derived from standard fire exposure, which is generally accepted as a non-realistic fire scenario, so questions of how relevant the charring rates are have come up. Modified charring rates for non-standard fire exposures such as Eurocode Parametric Fires have also been proposed (Hopkin et al 2011).

The codes also typically prescribe a zero-strength layer of $7 \mathrm{~mm}$ beyond the char depth that accounts for the loss of strength in the pyrolysis zone of the heated timber (see section 4.2.2 in CEN 2004a). The justification for this prescribed $7 \mathrm{~mm}$ zero-strength layer was given by Schaffer et al (1986). In this study, the layer was calculated using a $0.025 \mathrm{inch} / \mathrm{min}$ $(0.635 \mathrm{~mm} / \mathrm{min})$ charring rate and details of timber strength and elastic modulus beyond the char front from past experiments. The timber was assumed to have a constant reduced tensile strength and modulus of elasticity as a percentage of the ambient properties for 1.5 inches $(38.1 \mathrm{~mm})$ beyond the char front (simplified from the actual strength curves beyond the char front). Using a transformed section analysis, the strength of a simulated fire exposed beam with these reduced properties was computed and it was concluded that the strength was reduced by $20 \%$ compared to the use of ambient properties beyond the char layer. Thus, the effective reduced properties beyond the char front could be predicted by completely removing the strength of $20 \%$ of that reduced strength $(1.5 \mathrm{inch})$ layer; that is by neglecting the strength of an additional 0.3 inches $(7.52 \mathrm{~mm})$ beyond the char front. The only failure type considered was elastic behaviour until failure by tensile laminate rupture from bending. Additionally, the authors stated that "More independent experiments to test 
the adequacy of the beam simulation model for fire endurance are needed" (Schaffer et al. 1986). Thus, the accuracy of the $7 \mathrm{~mm}$ zero-strength layer for various scenarios or structure configurations may be questioned.

An in-depth study was done by Lange et al. (2015) in which numerous engineered timber beams were loaded in furnace tests exposed to standard and parametric fires. This research suggested, after a statistical study of the results, that the zero-strength layer beyond the char depth may range from $8 \mathrm{~mm}$ for a short, hot fire, up to $16 \mathrm{~mm}$ for a longer fire or standard fire exposure. This is significantly higher than the current code guidelines which simplify the calculation to $7 \mathrm{~mm}$.

Another phenomenon which has been observed in engineered timber is delamination. Delamination occurs when thermal penetration into the timber material interacts with an adhesive bondline. The adhesive strength degrades with temperature and the weight of the outer wood and char layer exceeds the adhesive's capacity, causing the layer to delaminate along the glue line. The occurrence has been observed most specifically with CLT, due to the large size and weight of the lamellae and surface area of exposed timber (see Su et al., 2018). There have been numerous studies on delamination in wood compartment fires but the occurrence of the phenomenon is highly variable. One study was able to show that in some cases, the delamination timing lined up with the char front reaching the first adhesive bondline (Hadden et al 2017). However, one case in that same study also suggested that delamination occurred when the char front had not yet reached the bondline, and the adhesive would have been at a much lower temperature than $300^{\circ} \mathrm{C}$ when it lost enough strength for the lamella to delaminate. Another study by NIST also showed that CLT walls and ceilings began to delaminate variably before and after the char layer had reached the 
adhesive layers (Su et al., 2018). Additionally, much more severe delamination was observed in compartments with less ventilation than a comparative well-ventilated compartment. This study has led to the investigation of novel adhesives that resist this delamination behaviour.

\subsubsection{Timber Adhesive Standards}

As aforementioned, current timber adhesive standards do exist for high-temperature performance. A summary of timber adhesive standards in Canada is shown in Figure 2.4. These standards are highly fragmented and do not necessarily test conditions that adhesive bonds in timber would be exposed to in a fire. For example, no standard exists that evaluates the performance of adhesive bondlines beneath the char layer in burnt engineered timber samples, but rather test the strength of the adhesive under uniform heating less than the charring temperature of wood. The main test method for evaluating the strength of adhesives in these standards is a shear test by compression loading on specimens that are $50 \mathrm{~mm}$ squared by $40 \mathrm{~mm}$ thick, with a slight offset on either side of the shear plane. This test is akin to the ASTM D905 test for evaluating the shear strength of adhesives (ASTM 2008).

Some of the most common adhesives used in manufactured engineered timber products in Canada include phenol-formaldehyde resorcinol resins (PRF) and polyurethane resins (PUR). PRF is a long-utilised structural adhesive in wood products and its related evaluation criteria is outlined in CSA-O112.7, developed in the 1970's. PUR is a newer adhesive and was developed after the release of these standards, but the testing and use of novel adhesives are approved under the more recent standards CSA-O112.9 and O112.10. The Canadian wood design standard CSA-O86 currently references to the Glulam product 
and manufacturer standards (CSA-O122-16). These, in turn, cite the CSA-O112-M series of standards for the evaluation of timber adhesives.

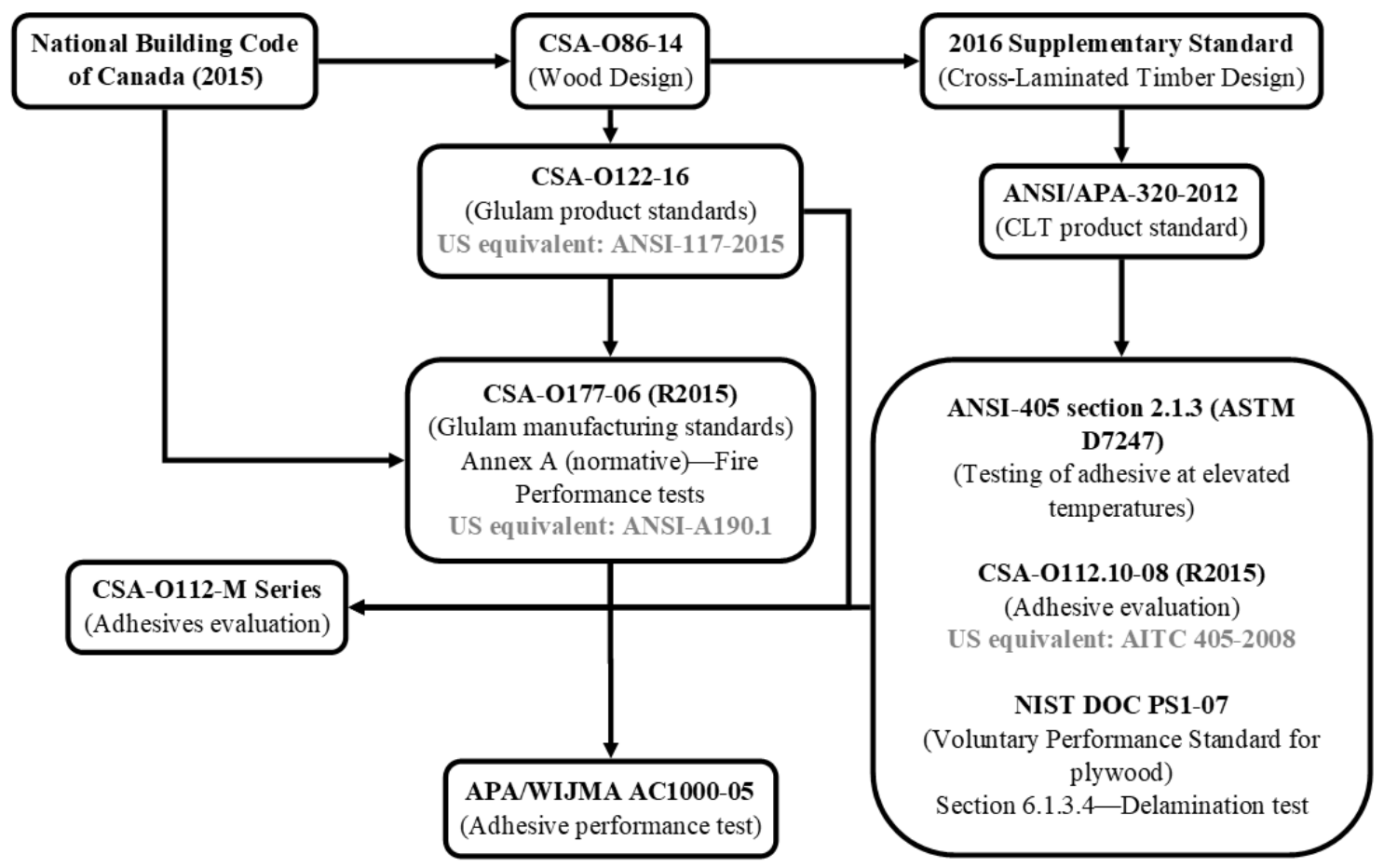

Figure 2.4: Canadian timber adhesives standards summary

The 2016 Supplementary Standard for CSA-O86 gave added guidance for cross-laminated timber (CLT), and points to ANSI/APA-320 for increased adhesive standards (ASTM 2008). This document gives provisions for Canadian adhesives to meet ASTM D7247 through ANSI-405 (ANSI and APA 2008). The ASTM D7247 standard test is meant to evaluate the shear strength of adhesives at elevated temperatures (ASTM 2016a), using an almost identical test specimen as in the CSA-O112-M series and ASTM D905. The test procedure involves heating several specimen sets of bonded and solid control samples to a bondline temperature of $220^{\circ} \mathrm{C}$ for a specified duration of time which depends on the product. Following the heating, the residual shear strength ratio for the bonded specimens must be at least equivalent to the lower $95 \%$ confidence interval for the results of the solid 
wood control specimens. ANSI/APA-320 also specifies that Canadian adhesives must meet section 6.1.3.4 of the NIST Voluntary Performance Standard DOC PS1 (NIST 2007). This test uses a Bunsen Burner flame exposure on a sloped surface of the specimen where several glue lines are exposed. After the test, the degree of delamination of the glue lines is visually inspected.

CSA-O177, the "Qualification Code for Manufacturers of Structural Glued-Laminated Timber" gives extra requirements for adhesives meeting CSA-O112.9 (CSA 2006), including PUR. Adhesives following this standard for evaluation of structural wood adhesives for exterior exposures must follow additional tests outlined in normative Annex A of the standard. In this section, a manufacturer has two options of test paths. In the first method, the products must pass a small-scale flame test akin to the DOC PS1 Bunsen Burner test, to assess delamination and glue line opening. Additionally, the products must pass APA/WIJMA AC1000, which was the first standard test meant to assess the performance of adhesives at a temperature near the ignition point of wood (Yeh et al 2005). It uses the same test procedure as ASTM D7247, which was originally published one year after APA/WIJMA AC1000. As of yet, the author is not aware of any standard tests that evaluate the performance of adhesive beneath the char layer in burnt engineered timber. Alternatively, in lieu of these two tests, the manufacturer may perform a full-scale fire test in conformance with CAN/ULC S101 to prove adequate fire performance. Adhesives meeting CSA-O112.7 (PRF) inherently meet bondline fire performance requirements.

\subsubsection{Research on Timber Adhesive Fire Performance}

A lack of research exists on the performance of adhesives used in engineered timber products in realistic fire conditions. For example, no previous tests have been done (to the 
knowledge of the author) where the samples being tested have been exposed to extreme heat and the char front has impinged on the glue line under examination. A number of experiments have been done on testing the effects of temperature on timber adhesives, however all of these procedures have tested the glue to temperatures below the charring temperature of wood, and typically consisted of homogeneous, steady-state temperatures achieved in an oven or environmental chamber. Adhesives in timber are typically tested at these temperatures below $300^{\circ} \mathrm{C}$ as the strength of the material is assumed to be completely lost at this point as the wood chars. However, this means that the performance of the adhesive just beyond a char front has not been studied.

Of the experiments mentioned above, notable research includes a study by Frangi et al. (2004) and Clauß et al. (2011). Frangi et al. tested small $40 \mathrm{~mm}$ bond lines on double lap specimens through compression loading for several different adhesives including one phenol-resorcinol-formaldehyde resin (PRF) and five different polyurethane adhesives (PUR). Hundreds of samples were heated in an oven to various temperatures ranging from ambient to $170^{\circ} \mathrm{C}$. It was found that the behaviour of the PUR adhesives varied greatly between manufacturer and thus chemical composition. Three PUR adhesives and the PRF adhesive performed similarly to that of the wood itself, while two of the PUR adhesives performed very poorly and began to lose strength from $50-70^{\circ} \mathrm{C}$. Clauß et al. similarly performed shear tests on several different adhesives but using single lap samples with bond lengths of only $10 \mathrm{~mm}$ through tensile loading. The specimens were uniformly heated up to $220^{\circ} \mathrm{C}$. The results in this test series were highly variable, but similarly found that the performance of various PUR adhesives were diverse with some losing thermal stability around $70^{\circ} \mathrm{C}$ while others remained stable until $150-200^{\circ} \mathrm{C}$. 
The most recent testing that specifically referenced to adhesive performance was a twopart test series by Nicolaidis et al. (2016) and Emberley et al. (2016) on glued single lap samples exposed to environmental chamber heating and CLT beams exposed to radiant heating, respectively. Pine and spruce wood were used, respectively, with a one-component PUR adhesive. The single lap samples had $600 \mathrm{~mm}$ bond lengths and were heated uniformly immediately prior to testing. The exposure on the CLT beams was akin to a realistic fire condition but at a very low heat flux of $6 \mathrm{~kW} / \mathrm{m}^{2}$, thus inducing a gradient of in-depth temperatures below the pyrolysis temperature of wood. The bond lines were mostly uniformly heated perpendicular to the bond on the tension side of the CLT beam, increasing the temperature of the bond lines to just $60-85^{\circ} \mathrm{C}$. Heating perpendicular to the bond line is a common scenario on CLT in realistic compartments, however Glulam members will often experience heating parallel to the exposed adhesive lines. In both parts of this experimental series, changes in the failure mode from primarily timber failures to primarily adhesive failures were seen in the higher temperature range tests. Of particular note, the failure modes in the adhesive were often made more severe by the discontinuity and stress concentrations caused by timber failure propagating into an adhesive joint. This stresses the importance of the size effect in experiments and failure modes changing based on the length of bond line tested. Su et al. (2018) recommend additional testing and developing new adhesives as illustrated in the recent compartment tests of CLT.

\subsection{Numerical Modelling of Timber in Fire Conditions}

Wood is an anisotropic material with a complex constitutive model and highly variable fracture mechanics due to natural imperfections and grain structure. Coupled with a heat transfer analysis where the thermal and mechanical properties both vary with temperature, 
the material presents an extreme challenge in the realm of numerical models. In the opinion of the author, there is currently no industry consensus on a realistic way to model timber in fire, especially for non-standard fire exposures.

Material thermal properties are required in all modelling applications involving heat transfer to or within a material, and furthermore, with structural applications, temperature dependent mechanical properties will also be required. Wood, being a naturally occurring material, is inherently prone to irregularities and imperfections. Although it has been studied for decades by countless academics and industry stakeholders alike, the understanding of how timber responds to fire is in its infancy due to these complexities. To date, there is no industry consensus on the number of chemical reactions and processes that take place during the pyrolysis of wood (König 2006, Roberts 1971). A recent effort to model the chemical processes of wood charring has shown promising results for predicting char at the mesoscale and offers a detailed review of various chemical process models that have been developed (Richter and Rein 2018). Heat transfer indicators temperature and mass loss rate for wood were inversely modelled using microscale experimental data, and the model was then validated at the mesoscale against cone calorimeter samples, fostering agreeable predictions compared to the experimental results.

Repetition is difficult and variations in results are high, and thus there is little industry consensus on the best way to model wood material properties. Additionally, due to the porous nature of wood and charcoal, convection and even radiation heat transfer modes may be present within the material itself, rather than only conduction which is the single mode of heat transfer in most other solids (König 2006). 
One method that has been used by academics in the past is to use a weighted combination of the thermal properties for the various components in the element in question (Fredlund 1993). The simplest method here would be to use an averaged value for the thermal conductivities or specific heats of wood and char. This in itself is complex as the thermal properties of wood itself depend on the species, density and moisture content, to start. Additionally, the material properties of charcoal cannot simply be used for the char layer as the density or porosity of that layer may not be the same as a simple lump of coal. Furthermore, if one were to truly use a full combination of the material properties, both volatile gases produced from the reaction and water (in liquid and vapour forms) should be included in some capacity. Possibly the most complicated factor in this method is that the mass fractions of all of these materials are constantly changing with time throughout the heating process.

One of the most widely accepted methods of modelling timber today is using effective material properties which encompass all of the material changes and mass movement empirically, such as those that are found in Part 1-2 of Eurocode 5, informative Annex B (CEN 2004b). The research that led to these effective values was largely pioneered by Jürgen König, who summarizes the background research and experimentation which has led to the Eurocode guidance in (König 2005). Therein, suggested effective values for thermal conductivity, specific heat, and density are given as functions of temperature, the relationships for which are shown in Figure 2.5. The values proposed here are calibrated based on test results from standard fire exposure (König 2005) and are meant to inherently incorporate all of the natural processes of wood turning to char, moisture and gas movement, and char oxidation and contraction. 


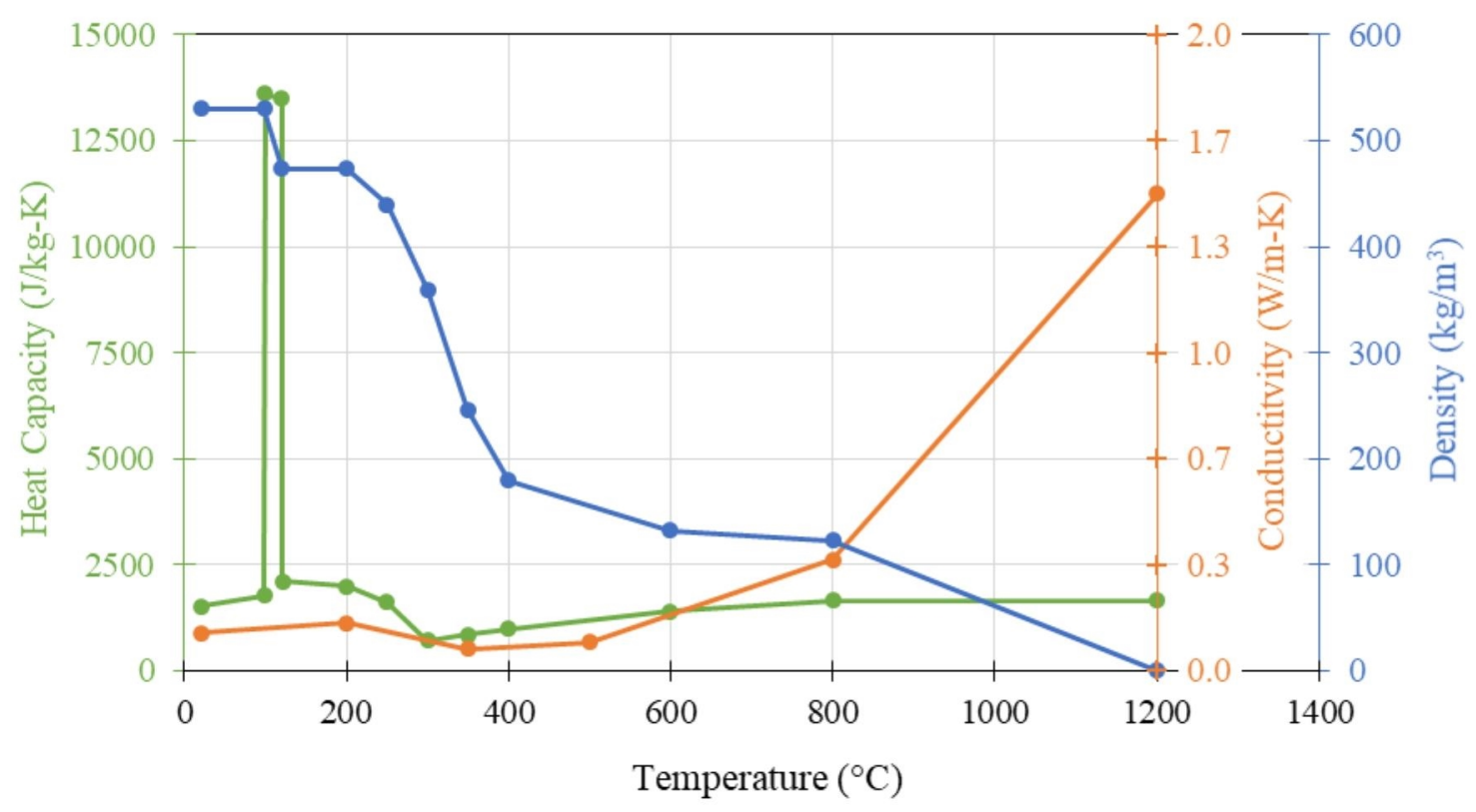

Figure 2.5: Eurocode EN 1995 Part 1-2 sample suggested effective thermal properties for wood (adapted from CEN 2004b)

The simplest way to understand the effective properties is to visualize a single element in a timber block undergoing one-dimensional heat transfer. The density at $99^{\circ} \mathrm{C}$ and below is equal to the wet density of the wood. As the element begins to heat up from $99^{\circ} \mathrm{C}$ to $120^{\circ} \mathrm{C}$ and moisture evaporates and moves away from that element, the density drops to the wood's dry density, and remains there until $200^{\circ} \mathrm{C}$. When the element temperature rises above $200^{\circ} \mathrm{C}$ the density begins to decrease further as wood turns to less dense char through pyrolysis and continues to decrease to a non-physical density of zero at $1200^{\circ} \mathrm{C}$, when there is no longer any material within that element as the char has contracted or oxidized away. In the same way, the conductivity for that element will first increase as moisture begins to move through the wood fibres carrying heat with it, and then decrease as char begins to form after $200^{\circ} \mathrm{C}$. Then, beyond a certain point, the conductivity begins to rise again as the char layer begins to crack and contract, as the true heat transfer through the element would be increased from convection or radiation. A curve for the changes in specific heat is also 
given in a similar manner, but with a distinctive jump in the curve between $99^{\circ} \mathrm{C}$ and $120^{\circ} \mathrm{C}$. This jump is to account for the extra energy required to evaporate the water in the timber before heating up the material itself.

Another technique used to model the evaporation of moisture within the timber is the latent heat method, which may be used instead of a jump in the effective specific heat curve. The latent heat method simply involves programming the consumption of energy through the latent heat of vaporization of water into the model which is activated at the phase change temperature of $100^{\circ} \mathrm{C}$ (Buchanan and $\left.\mathrm{Abu} 2017\right)$. In sophisticated models which actually attempt to incorporate a mass balance, the sign of this phase change becomes important as after moisture evaporates, a portion will move deeper into the unheated zone of the wood, re-condensing once it drops below the phase change temperature (Fredlund 1993).

A large limitation of the above methods, however, is that the values mentioned are only calibrated to standard fire exposure. It is widely accepted that the charring behaviour of wood varies with fire severity or heat exposure, so the properties that inherently account for experimental charring behaviour can clearly not be extended for application in modelling natural fires. This is an issue as, in advanced calculations for structural fire engineering which require these material properties, the practitioner will almost never wish to model a standard fire exposure as it is not realistic. Additionally, the suggested properties should not be used for the cooling phase of a fire (König 2006). Finally, it has also been suggested that thermal conductivity and heat capacity should be corrected for density and wood moisture content (Cachim and Franssen 2009). Evidently, there are various options for modelling heat transfer in wood all of which will result in different effective thermal properties. Recently, Hopkins et al. (2011) presented a suggested formulation for effective 
conductivity in the more realistic Eurocode parametric fire curves, which were implemented in the finite element software DIANA and the results compared to code recommended parametric fire charring rates.

Aside from thermal material properties and changing density, wood's strength and stiffness will also change with heat and changes in moisture content which must be considered in coupled heat transfer and structural analyses. Additional characteristics that have been greatly studied and debated are the ignition and flame spread characteristics of wood. It has been shown that the ignition of wood is very hard to predict, especially because results vary significantly depending on experimental set up. An in-depth review of the available literature was given by Babrauskas (2001), in which several cases of varying heat fluxes and auto or piloted ignition are considered. Furthermore, it is stated that ignition temperature is really a fictitious concept (a simplification of much more complex factors) developed because it is easily applied in a design scenario. Similarly, flame spread has been the subject of study of many research programs. Flame spread is found to vary significantly based on a number of factors such as ventilation or geometry, and is further complicated by the natural variability in wood. A comprehensive theoretical guide to calculating flame spread on thermally thick materials is given by Thomas (1995).

Thus far, advanced calculation methods for structural fire engineering analysis of timber elements are still in development, largely due to this lack of thermal property validation. Several experimental research study programmes have included finite element modelling of the tests, however, as a step towards a future of full wood structural fire design. There are several commercial software packages available for structural fire engineering (SFE). Some of these computer programs were developed especially for SFE applications, such 
as SAFIR or Vulcan, which integrally include thermal and structural analyses. Additionally, there are general purpose programs such as Abaqus which include both heat transfer and mechanical analysis capabilities. A study was done by Werther et al. (2012) comparing the capabilities of the aforementioned software packages for modelling heat transfer in timber, which is summarized in Section 2.2.1.3.

Typical structural fire models can be run as sequential analyses, in which the time-history data from a thermal analysis is input as a field variable for the subsequent mechanical analysis, or fully coupled analyses, in which the temperatures and structural response are calculated simultaneously in each time step. The latter is more computationally demanding but is required if the structural response and deformations affect the heat transfer to the element. Because timber thermal properties are so highly simplified, and structural simulations are typically done for uniform temperature exposure, the structural response would not affect the thermal analysis. However, if more sophisticated models were implemented, timber responses such as cracking and char fall-off could affect the heat transfer to the structure. Yet, the use of finite element modelling of timber in fire is also limited by the lack of expertise in simple ambient structural modelling of timber. This complex material can be modelled quite well with orthotropic engineering constant elastic material properties, and is often modelled in the fully elastic range (Van Beerschoten 2013). In ambient testing, this is generally acceptable as the main failure mode is rupture in tension, in which the constitutive model for timber remains linear-elastic until failure. However, in a fire scenario when strengths and stiffness are reduced, this likely does not hold true and some sort of plasticity or damaged material models may be required. The 
fracture behaviour of timber requires highly detailed models and the nonlinear, anisotropic material data is not readily available.

\subsubsection{Past Numerical Modelling of Timber}

Several attempts have been made in the past to model wood in Abaqus, both in ambient and fire conditions. Studies of past numerical models are reviewed for selection of material models and material property parameters.

\subsubsection{Van Beerschoten (2013)}

Van Beerschoten's thesis was a study on the gravity performance of PT timber beams in which experiments and numerical modelling was involved. Four large-scale PT timber beams were tested with various cross-sections and tendon schemes. Additionally, extensive ambient testing on LVL in order to determine the required material properties for numerical modelling was done. Table 2.2 gives a summary of the material parameters determined for use with the LVL specimens tested. Van Beerschoten discussed in great detail the test methods and results for determining the elastic moduli and constitutive models, Poisson's ratios, shear moduli and strength parameters for the LVL specimens in three material directions, along with the implementation of these parameters into a numerical model in Abaqus. Due to the manufacturing process of LVL, structural elements may be defined by three orthogonal directions: the longitudinal axis is aligned parallel with the wood grain, one transverse axis is tangential to the original tree trunk rings, and the other is normal to the rings (that is, aligned to the radial direction - see Figure 2.1b). Van Beerschoten employed an orthotropic elastic material model for most of his simulations as they largely remained in the elastic region until brittle tensile failure. In one of the four PT timber beams which were tested and modelled there was a longitudinal compressive failure in some of 
the wood fibres, so isotropic hardening plasticity was applied with a developed nonlinear constitutive model for compression. This was justified as the stresses in the other grain directions and in tension did not reach their respective yield stresses, so the plasticity model was only engaged in the applicable stress region.

Table 2.2: Resulting material parameters from Van Beerschoten experimental testing of LVL (Van Beerschoten 2013)

\begin{tabular}{|c|c|c|c|c|c|}
\hline $\begin{array}{c}\mathbf{E}_{\mathbf{L}} \\
(\mathbf{M P a})\end{array}$ & $\begin{array}{c}\mathbf{E}_{\mathbf{T}} \\
(\mathbf{M P a})\end{array}$ & $\begin{array}{c}\mathbf{E}_{\mathbf{R}} \\
(\mathbf{M P a})\end{array}$ & $\begin{array}{c}\mathbf{G}_{\mathrm{LT}} \\
(\mathbf{M P a})\end{array}$ & $\begin{array}{c}\mathbf{G}_{\mathbf{L R}} \\
(\mathbf{M P a})\end{array}$ & $\begin{array}{c}\mathbf{G}_{\mathrm{TR}} \\
(\mathbf{M P a})\end{array}$ \\
\hline 12157 & 426 & 371 & 856 & 901 & 96 \\
\hline $\boldsymbol{v}_{\mathbf{L T}}$ & $\boldsymbol{v}_{\mathrm{TL}}$ & $\boldsymbol{v}_{\mathrm{LR}}$ & $\boldsymbol{v}_{\mathrm{RL}}$ & $\boldsymbol{v}_{\mathrm{TR}}$ & $\boldsymbol{v}_{\mathrm{RT}}$ \\
\hline 0.59 & 0.02 & 0.48 & 0.02 & 0.22 & 0.14 \\
\hline
\end{tabular}

\subsubsection{Menis (2012)}

This study was comprised of experimental testing and numerical modelling (in Abaqus) of LVL tension members and cross-laminated timber (CLT) bending elements. In order to undertake the modelling of wood in Abaqus, Menis first compared the results of an isotropic elastic material model to those of an orthotropic elastic material model. Due to the nature of the experiments, it was determined that the isotropic and orthotropic material models had negligible differences, corroborating the use of the isotropic model. It was desirable to use the isotropic model as it was compatible with a plasticity model which allowed for asymmetric constitutive models. This material is known in Abaqus as the Concrete Damaged Plasticity (CDP) model, which was developed to represent the material behaviour of concrete. Fundamentally, the constitutive behaviour of wood may be represented in a similar manner to concrete, in which the compressive stresses cause 
crushing leading to softening and damage (elasto-plastic), while the tensile stresses result in a linear elastic behaviour until rupture (elasto-brittle). In heat exposure especially, the behaviour of wood in compression or tension are quite different and are affected by temperature in different ways, according to Eurocode 5 Part 1-2 (CEN 2004b). Thus, it was desirable to use the available material models which allowed for the definition of these key differences. The aim of the study was to quantify and model the fire resistance of various structural elements, experimentally and numerically. The findings were agreeable with the results of the experiments in terms of fire resistance times and reasonably comparable deflection behaviour.

\subsubsection{Werther et al. (2012)}

Werther undertook a study of modelling heat transfer in wood using various finite element software packages. The results using different methods of accounting for moisture evaporation were compared, including an effective specific heat method and a latent heat method. Effective specific heat values as a function of temperature are defined in Eurocode 5 Part 1-2 with a jump in the values at $100^{\circ} \mathrm{C}$ to account for the additional energy required to evaporate water at this temperature, as described in Section 2.2. The latent heat method, rather, eliminates this discontinuity in the specific heat, but adds the input of a latent energy of evaporation for the total mass of water in the wood. Additionally, a mesh sensitivity study was completed. The study found that results are more stable using a latent heat method, and all software packages converge to the same results with a small enough mesh size. It was recommended that a $3 \mathrm{~mm}$ mesh is the optimal element size for accurate results and computational efficiency. 


\subsection{Post-Tensioned Timber}

As a result of the advancement of engineered timber products and the continual increase of sustainability mindfulness of society, new and innovative timber structural systems have been proposed in order to maximize the efficiency and potential application of timber in commercial construction. One of such systems is post-tensioned (PT) timber, developed and researched over the past decade by researchers at the University of Canterbury in New Zealand, having been proposed as an enhanced timber construction system for seismic performance (Palermo et al. 2005). The system consists of engineered timber structural elements which may include beams, columns and walls, combined with high-strength steel tendons which place the wood members in a state of pre-stress. The technology has been commonly used in concrete and allows for increased structural efficiency. Beams may have longer spans or smaller cross-sections for the same loading due to increased capacity. Entire building systems become more ductile and self-centering, resulting in highly effective seismic performance. The merits of the structure have already been proven and the system is gaining traction worldwide, with proposed buildings even in North America. It has been implemented in numerous buildings worldwide, including seven buildings in New Zealand. Of these buildings, all but two have automatic sprinkler systems installed and none of them have fire protection on the PT system elements.

As post-tensioned (PT) timber is an innovative building system, limited research has been conducted over the past decade in order to validate its performance in fire. This section provides background on the development and importance of the PT timber system, along with a summary of its implementation in structures internationally. The past work of experimentalists in structural fire engineering on PT timber are detailed to serve as a basis 
for this research. Experiments have been done on the system's fire performance by (Spellman 2012) and (Costello 2013) which are discussed in Section 2.3.2.

\subsubsection{Post-Tensioned Timber Beams}

This research study focuses on the fire performance of PT timber beams specifically. These beams are typically constructed with a box-beam built up of two web and two flange engineered timber members with a central cavity for the post-tensioning steel tendons. A typical cross-section and beam schematic are shown in Figure 2.6. The steel tendons are typically tied at the ends of the beam with a steel anchoring system, at which one end is fixed and one end may be jacked to tighten the strand to a given tensile force.
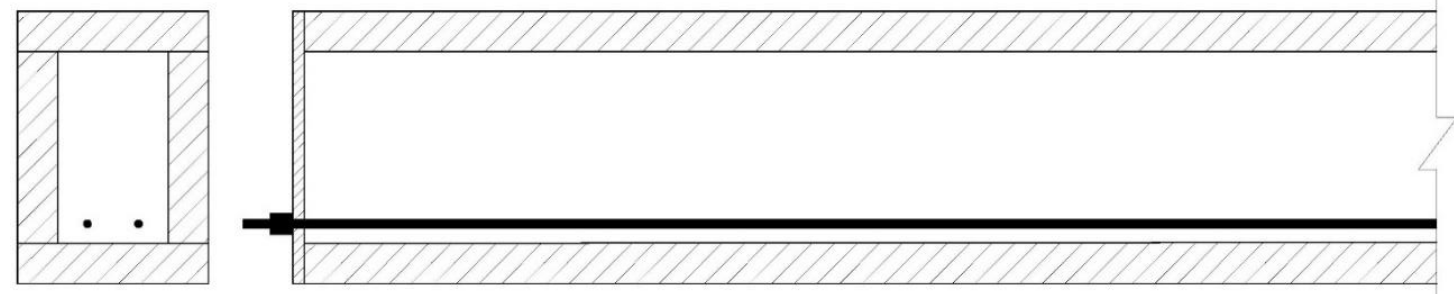

Figure 2.6: Schematic of typical PT timber beam cross-section showing built up wood webs and flanges and high-strength steel tendons, along with section cut through beam length showing tendon anchorage

In fire, the PT steel tendons tend to lose some restraint due to thermal expansion and charring of the wood beam. It has been recommended as a design strategy for PT timber in fire to conservatively neglect the steel tendon contribution to the beam capacity. However, consideration of the tendon may significantly increase design efficiency. A simplified analytical design method was proposed for PT timber beams in fire following the previously mentioned experiments. This method may include consideration of the changing tendon force due to thermal and nonlinear geometric effects and the changing cross-sectional properties due to charring. The simplified design method uses the ambient 
beam design and the required standard fire duration for fire resistance to calculate the remaining beam capacity, or calculates the behaviour at each time step until the total failure load and time is reached if such detail is required.

\subsubsection{Past Experiments on PT Timber in Fire}

Several experiments have been done on PT timber beams in ambient and fire conditions at the University of Canterbury. The current study focusses on the fire performance of the PT timber beams, in order to validate a numerical model of the experiments. Thus, the experiments reviewed herein are the works of Spellman (2012) and Costello (2013). The data comparisons for these experiments was done in collaboration with researchers at the University of Canterbury who were part of the team performing the experiments.

\subsubsection{Spellman (2012)}

In order to investigate the performance and failure mechanisms of post-tensioned timber beams in fire, Spellman performed three large-scale furnace tests, along with several anchorage fire protection tests. The beams were loaded under four-point bending with a clear length of $4 \mathrm{~m}$ inside an ISO 834 standard test furnace, and a center-to-center span of $4.36 \mathrm{~m}$ on $100 \mathrm{~mm}$-wide steel support plates. The two point loads were applied symmetrically with a $1.5 \mathrm{~m}$ spreader beam. The cross-sections of the tested beams are shown in Figure 2.7, and an overview of the test parameters is given in Table 2.3. Spellman's box-beams were built up of laminated veneer lumber (LVL) sections and were loaded at a constant level of $40 \mathrm{kN}$ throughout fire exposure, approximately $49 \%$ of their ambient capacity. The steel tendons used for post-tensioning were 7 -strand wires with a nominal area of $100 \mathrm{~mm}^{2}$ and were loaded within their elastic range. 


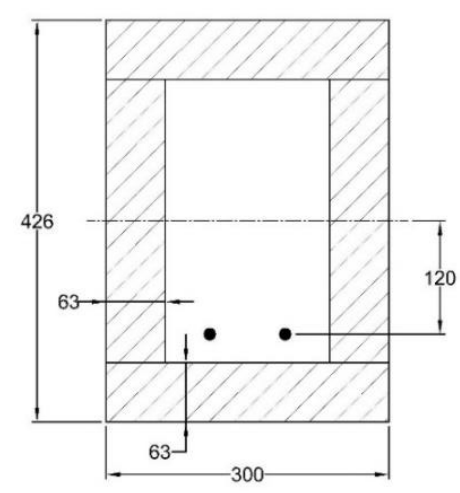

BEAM A

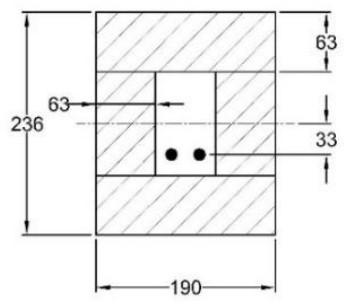

BEAM B

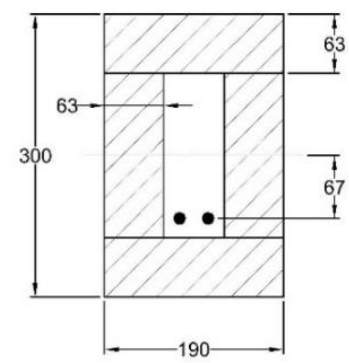

BEAM C

Figure 2.7: Overview of beam cross-sections for Spellman's tests (Spellman 2012) outlined in Table 2.3 below (all dimensions in millimeters)

Table 2.3: Summary of tests completed by Spellman (2012)

\begin{tabular}{|c|c|c|c|c|}
\hline Beam & $\begin{array}{c}\text { Fire } \\
\text { Exposure }\end{array}$ & $\begin{array}{c}\text { Beam Span } \\
(\mathbf{m})\end{array}$ & $\begin{array}{c}\text { Spreader-Beam Span } \\
(\mathbf{m})\end{array}$ & $\begin{array}{c}\text { Tendon Force } \\
(\mathbf{k N})\end{array}$ \\
\hline A & ISO 834 & 4.36 & 1.5 & 213 (total) \\
\hline B & ISO 834 & 4.36 & 1.5 & 213 (total) \\
\hline C & ISO 834 & 4.36 & 1.5 & 232 (total) \\
\hline
\end{tabular}

The beams were each designed for different failure modes, and while Beams A and C performed as expected, Beam B failed prematurely (likely due to pre-existing defects). Beam A was deemed to fail at a time of approximately 64 minutes when runaway deflections occurred, and two longitudinal cracks were seen to have formed into the shear region of the beam web. Beam $\mathrm{C}$ failed at the time of approximately 56 minutes when runaway deflections occurred. The failure mechanism for this beam was the formation of two plastic hinges at the boundaries of the shear region, first under one of the loading plates and second just inside the support. Additionally, a localized crack and buckling occurred in the web within this shear region. 


\subsubsection{Costello (2013)}

Spellman recommended further investigation of shear failure in PT beams after his experiments and as such, Costello's research was commissioned. Costello performed several large-scale tests of timber box-beams, some with post-tensioning steel strands and some without. One of the tests was done in a furnace with a standard fire exposure, and four additional tests were done at ambient temperature with a reduced cross-section to simulate loss of wood to charring. The test specimens were made from LVL and the beam span and section varied in each test. The loading schemes were all four-point bending tests with varying spreader-beam spans. The specified spans were assumed to be the center-tocenter distance of the supports or loading plates, except for the furnace test where the specified beam span was the clear span inside the furnace. In the ambient tests the load was ramped up linearly until beam failure, while in the furnace test the load was held constant over the fire duration until failure. In order to investigate shear failure (as was suggested after Spellman's tests), the beams were each designed to fail in shear. An overview of the tests completed by Costello is shown in Figure 2.8 and Table 2.4. Beams 2 and 4 were milled down to a manually-reduced cross-section size to replicate the expected char depths from Beams 1 and 3, respectively. 


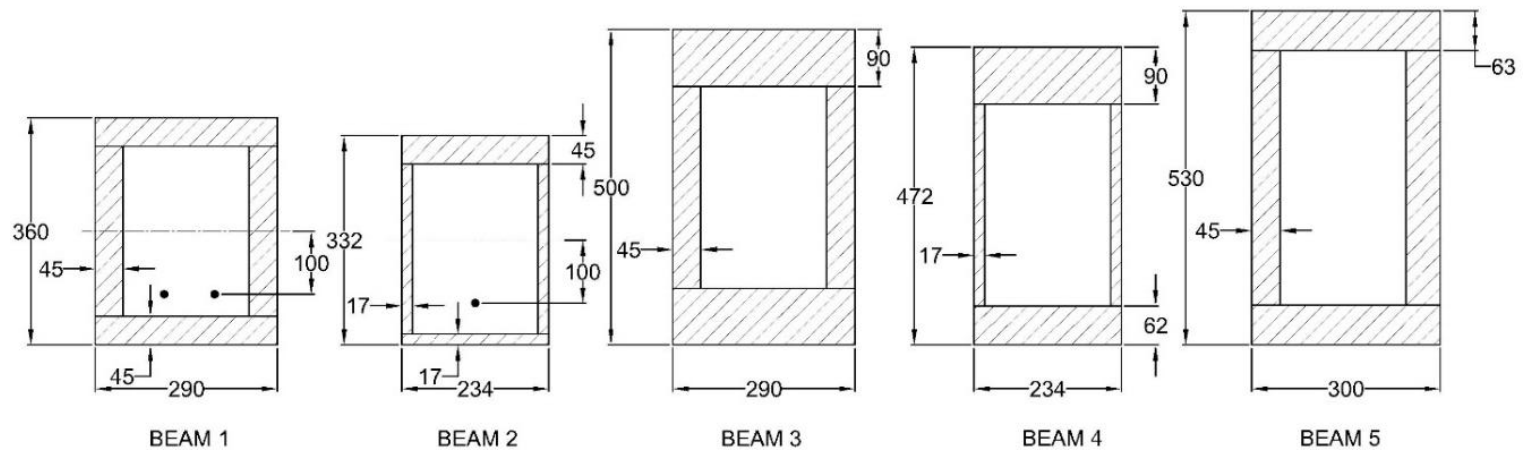

Figure 2.8: Overview of beam cross-sections for Costello's tests (Costello 2013) outlined in Table 2.4 below (all dimensions in millimeters)

Table 2.4: Summary of tests completed by Costello (2013)

\begin{tabular}{|c|c|c|c|c|}
\hline Beam & $\begin{array}{c}\text { Fire } \\
\text { Exposure }\end{array}$ & $\begin{array}{c}\text { Beam Span } \\
(\mathbf{m})\end{array}$ & $\begin{array}{c}\text { Spreader-Beam Span } \\
(\mathbf{m})\end{array}$ & $\begin{array}{c}\text { Tendon Force } \\
(\mathbf{k N})\end{array}$ \\
\hline 1 & ISO 834 & 4.2 & 2.5 & 107 (total) \\
\hline 2 & none & 3.64 & 2.48 & 94 \\
\hline 3 & none & 3.64 & 1.71 & none \\
\hline 4 & none & 3.64 & 1.71 & none \\
\hline 5 & none & 2.82 & 1.25 & none \\
\hline
\end{tabular}

Costello's first beam which was tested in the furnace was loaded to a total of $100 \mathrm{kN}$ before the fire exposure and was held at this constant load throughout the fire until failure, which is roughly $25 \%$ of the beam's ambient shear capacity. The failure mechanism observed was plastic hinges which formed at the boundaries of the shear region (similar to Spellman's Beam $\mathrm{C}$ failure) of one side of the beam. The mid-span deflection measured at failure was $25 \mathrm{~mm}$, which occurred 29 minutes after the beginning of the fire exposure. Additionally, several longitudinal shear cracks propagated throughout the depth of the section below the support where the failure occurred. The average char depth at the end of the fire exposure was approximately $25 \mathrm{~mm}$ in both the webs and bottom flange. The steel tendons had a nominal cross-sectional area of $100 \mathrm{~mm}^{2}$ each and a yield strength of $1520 \mathrm{MPa}$. Thus, the tendon forces result in stress levels within the linear elastic range. 
The failure mechanisms in all of the ambient beams manifested as longitudinal shear cracks, which typically developed from the end of the beam and remained in the shear regions. The failure loads and resulting deflections for each of these tests are summarized in Table 2.5. Similarly to Beam 1, the steel tendon in Beam 2 was loaded within the linear elastic range.

Table 2.5: Summary of failure loads and corresponding mid-span deflections from Costello's ambient tests (Costello 2013)

\begin{tabular}{|c|c|c|}
\hline $\begin{array}{c}\text { Test } \\
\text { Number }\end{array}$ & $\begin{array}{c}\text { Failure Load } \\
(\mathbf{k N})\end{array}$ & $\begin{array}{c}\text { Mid-span Deflection } \\
(\mathbf{m m})\end{array}$ \\
\hline 2 & 138 & 22 \\
\hline 3 & 473 & 24 \\
\hline 4 & 147 & 16 \\
\hline 5 & 515 & 21 \\
\hline
\end{tabular}

\subsubsection{Existing and Proposed PT Timber Structures}

Beginning with New Zealand, the home country of PT timber, many structures have already been approved and built for various building functions. Initially, PT beams were employed in a pool facility following research and testing done at the University of Canterbury (UC). Later, a medium-scale test structure was built at UC which was later converted into a small office space for EXPAN, the patent-holders and leading researchers of PT timber. The structure had withstood several rounds of simulated earthquake testing in the lab before enduring the devastating 2011 Christchurch earthquake after its office conversion on the UC grounds, during which it was deemed "the safest place on campus" (NZ Wood 2013).

The first structure to be approved and built using the Pres-Lam PT timber system outside this research group was the Arts and Media Centre of Nelson-Marlborough Institute of 
Technology (NMIT) in Nelson, New Zealand which was completed in 2011. This structure made use of post-tensioning in LVL shear wall panels. Currently, there are at least eight PT timber buildings in New Zealand, which are summarized in Table 2.6. Many innovative uses of the technology have been incorporated into these buildings. For example, the St. Elmo Courts building uses hybrid concrete-timber moment-resisting frames, where the columns are concrete. The beams are PT timber, where the post-tensioning spans through the concrete columns across multiple bays. The Kaikoura District Council Building is the most recent innovative construction and houses many functionalities such as offices, a library and a museum, and was the first building ever constructed using the PT technology in CLT.

Outside of New Zealand, a handful of PT timber buildings have been proposed and built. The House of Natural Resources (HoNR) research centre at the Swiss Federal Institute of Technology (ETH) in Zurich was completed in 2015 and made use of PT timber technology in a hardwood structural frame (ETH Zürich 2015). Additionally, two PT timber buildings are planned in Oregon, the Framework Project in Portland and the Peavy Building at Oregon State University (Iqbal and Popovski 2017). There is one PT timber building in the planning phase in Ottawa, proposed for the Cathedral Hill 2 project (Iqbal and Popovski 2017). These buildings intend to use PT technology largely in the lateral force-resisting systems. 
Table 2.6: List of PT timber buildings in New Zealand

\begin{tabular}{|c|c|c|c|}
\hline Building Name & Location & Completed & PT Timber Use \\
\hline $\begin{array}{l}\text { Diocesan Aquatic } \\
\text { Centre }\end{array}$ & $\begin{array}{l}\text { Epsom (Near } \\
\text { Auckland) }\end{array}$ & 2009 & $\begin{array}{l}\text { PT LVL roof main beams and } \\
\text { rafters a }\end{array}$ \\
\hline EXPAN Office & $\begin{array}{l}\text { University of } \\
\text { Canterbury, } \\
\text { Christchurch }\end{array}$ & $\begin{array}{l}2011 \\
\text { (Since } \\
\text { dismantled) }\end{array}$ & $\begin{array}{l}\text { Moment-resisting frames (PT } \\
\text { LVL beams) and shear wall } \\
\text { panels b }\end{array}$ \\
\hline $\begin{array}{l}\text { NMIT Arts and } \\
\text { Media Centre }\end{array}$ & Nelson & 2011 & $\begin{array}{l}\text { Coupled LVL shear wall } \\
\text { panels }{ }^{c}\end{array}$ \\
\hline $\begin{array}{l}\text { Carterton Events } \\
\text { Centre }\end{array}$ & $\begin{array}{l}\text { Carterton (North } \\
\text { of Wellington) }\end{array}$ & 2011 & $\begin{array}{l}\text { Coupled LVL shear wall } \\
\text { panels similar to NMIT }\end{array}$ \\
\hline $\begin{array}{l}\text { Nikau office } \\
\text { building at BRANZ }\end{array}$ & Wellington & 2011 & $\begin{array}{l}\text { LVL portal frame with PT } \\
\text { columns for lateral resistance }^{d}\end{array}$ \\
\hline $\begin{array}{l}\text { Massey University } \\
\text { CoCA Building }\end{array}$ & Wellington & 2012 & $\begin{array}{l}\text { Moment-resisting frames (PT } \\
\text { beams and columns with } \\
\text { exposed tendons) in } \\
\text { conjunction with PT concrete } \\
\text { shear walls b }\end{array}$ \\
\hline \begin{tabular}{|l|} 
Young Hunter \\
House (Merritt \\
Building) \\
\end{tabular} & Christchurch & 2013 & $\begin{array}{l}\text { Moment-resisting frames (PT } \\
\text { beams with zero eccentricity } \\
\text { and exposed anchorage) } \mathrm{e}\end{array}$ \\
\hline $\begin{array}{l}\text { Trimble Navigation } \\
\text { Building }\end{array}$ & Christchurch & 2014 & $\begin{array}{l}\text { Moment-resisting frames (PT } \\
\text { beams only on first level) and } \\
\text { shear wall panels }{ }^{b}\end{array}$ \\
\hline $\begin{array}{l}\text { Kaikoura District } \\
\text { Council Building } \\
\end{array}$ & Kaikoura & 2014 & Shear walls (PT CLT panels) ${ }^{\mathrm{f}}$ \\
\hline $\begin{array}{l}\text { St. Elmo Courts } \\
\text { Building }\end{array}$ & Christchurch & 2015 & $\begin{array}{l}\text { Moment-resisting hybrid } \\
\text { frames (multi-bay PT beams } \\
\text { with draped tendons and } \\
\text { concrete columns) }^{\mathrm{b}} \\
\end{array}$ \\
\hline \multicolumn{4}{|c|}{$\begin{array}{l}\text { a (Carter Holt Harvey Group 2010) } \\
\text { b (Costello 2013) } \\
\text { c (Holden et al 2016) } \\
\text { d (McGechie 2011) } \\
\text { e (Kirk Roberts Consulting Engineers 2014) } \\
\text { f (XLam 2017) }\end{array}$} \\
\hline
\end{tabular}

\subsection{Conclusions}

Evidently, there have been decades of research on the various complexities of timber and how it behaves in fire. The background information provided along with the review of 
available literature and past research indicates that there are still many gaps in the knowledge. More details and data are required to confidently understand and design innovative timber structures for fire safety. Numerous structures have been built internationally in a conservative way so that public safety is ensured. However, the ability to design these novel structures with efficiency and proof of adequacy relies on an intimate knowledge of the material which is still uncertain in areas such as timber adhesives. Furthermore, the designs for these systems in Canada may benefit from validated numerical models to defend them. Past efforts in modelling timber have not captured the behaviour necessary to capture the failure of PT timber beams in fire, and thus the development of a new model is required. 


\section{Chapter 3: Finite Element Modelling of Post-Tensioned}

\section{Timber Beams}

\subsection{Introduction}

In recent years the popularity of timber as a commercial building material has greatly increased. Today, the benefits of large-scale massive timber construction include savings in weight, labour, construction time, and carbon footprint compared to other building materials. Accordingly, the use of engineered massive timber products is becoming increasingly promoted in the construction of mid- to high-rise structures. A full summary of tall massive timber buildings in the world as of June 2017 is given by CTBUH (2017), in which almost 50 completed, proposed or under-construction buildings are listed. To achieve these goals of increased massive wood structures, new timber construction techniques are being rapidly developed. Historically, however, timber has been focused on primarily as combustible construction. This necessitates research to develop confidence in the fire safety of these novel technologies.

Fire resistance experiments have been done for decades on all building materials and assemblies. In Canada, building components and assemblies must be tested under standard fire exposure in order to demonstrate equivalency with approved fire code regulations, which was discussed in Section 2.1.2. However, more realistic fire tests are of particular importance to combustible materials as they interact with the fire and often have exposuredependant properties.

Not only are the mechanical properties of wood affected by elevated temperatures, but the material itself changes to char and eventually oxidizes away. Furthermore, the naturally 
occurring material has more variable or unpredictable properties as opposed to a synthetically manufactured material. Due to this complexity and combustibility, timber is typically dealt with in fire in a very conservative way. Current common practice for the fire engineering design of timber structures is either to encapsulate the timber to prevent direct fire exposure or the sacrificial char method. The latter is used for exposed timber in which a "sacrificial" thickness of timber beyond what is required for structural fire loading is added to the timber cross-section which is expected to char away in the event of a fire. The remaining wood beneath the char layer is then assumed to have the full ambient mechanical properties of the original material. Charring rates have been determined and code-accepted for several wood species, densities, or engineered wood products under standard fire exposure.

For increasingly complex timber construction systems, the sacrificial char method may be lacking in detail or accuracy for whole structure response. In the case where such detail is required, it may be beneficial to use the finite element method (FEM) to predict the mechanical behaviour of a structure. FEM uses energy-based equations to represent the physics of structural mechanics, in order to approximate structural performance. These models can be very complex, including multifaceted material properties which may be defined as functions of temperature. Validating numerical models of innovative structural systems is important from an industry perspective as the FEM models may be used as an engineering tool to allow for more confidence in optimized performance-based designs rather than highly conservative prescriptive solutions.

Post-tensioned (PT) timber, or Pres-Lam, is a novel timber construction system which has been developed and tested over the past decade at the University of Canterbury in New 
Zealand. The system utilizes a technology developed originally for concrete to increase span potential and seismic performance. Implementing the PT technology in timber can result in similarly increased performance. Limited development on numerical models has been done on the system for the optimization of design for fire performance. It is desirable to validate a numerical model for the system to increase opportunity to adopt the system in jurisdictions such as Canada. The development of an FEM model for PT timber systems is beneficial to industry as there is currently no analysis technology available to practitioners to predict the system behaviour in ambient or fire conditions, and a validated FEM model is the first step in creating an accessible tool for designers.

The overarching goal of this research is to facilitate the use of PT timber in Canada. This component aims to substantiate this goal by developing a numerical FEM model for thermo-mechanical analysis of PT timber beams using Abaqus, validated by data from past experiments (Spellman 2012, Costello 2013). PT timber has been fabricated primarily from laminated veneer lumber (LVL) in NZ, however Glulam is more readily available in a larger range of sizes in Canada. Herein, the numerical model will be developed around a more general engineered wood product rather than specifically LVL.

\subsection{Research Objectives and Approach}

The main objective of this chapter is to develop an accurate numerical model which is capable of demonstrating the behaviour of PT timber beams in ambient and fire exposure. Thus, the numerical model implemented should have verification and validation with experiments at both ambient and elevated temperature conditions. 
Post-tensioned timber systems may be constructed from various wood products, and are typically built from LVL or Glulam. It was desired to create a model which will be applicable for both products. Often LVL is modelled with three orthotropic directions: longitudinal, tangential and radial, according to the growth rings (as shown in Figure 2.1b). This is due to the manufacturing process where the veneers are cut tangential to the growth rings and thus the thickness is always in the radial direction. Detailed wood properties typically have different values for all three of these dimensions. However, Glulam is manufactured in such a way that the longitudinal direction is always parallel to the wood grain, but the orientation of the laminates in the transverse directions is random. This is due to the way the laminates are cut from log cross-sections (which was shown in Figure 2.1a). Thus, the wood should be modelled herein as transversely isotropic in order to maintain generality for all wood products.

In order to validate the numerical model, a series of past experiments are simulated using Abaqus finite element analysis software (the selection of which is detailed in the following section). The modelling plan consists of purely ambient mechanical analyses, thermal analyses, and fire-exposed mechanical analyses with thermal results input as predefined fields. The modelling plan is outlined below in Table 3.1. More detailed descriptions of the experiments were provided in Section 2.3.2. 
Table 3.1: Outline of tests to be modelled, with more detailed descriptions of the past experiments in Section 2.3.2

\begin{tabular}{|l|l|l|}
\hline Model & Study & Experiment Description \\
\hline C1 & $\begin{array}{l}\text { Costello } \\
(2013)\end{array}$ & $\begin{array}{l}\text { Beam 3 -ambient test of unmodified beam cross- } \\
\text { section without PT tendons }\end{array}$ \\
\hline C2 & $\begin{array}{l}\text { Costello } \\
(2013)\end{array}$ & $\begin{array}{l}\text { Beam 4 - ambient test of milled beam cross- } \\
\text { section without PT tendons }\end{array}$ \\
\hline C3 & $\begin{array}{l}\text { Costello } \\
(2013)\end{array}$ & $\begin{array}{l}\text { Beam 5 - ambient test of unmodified beam cross- } \\
\text { section without PT tendons }\end{array}$ \\
\hline C4 & $\begin{array}{l}\text { Costello } \\
(2013)\end{array}$ & $\begin{array}{l}\text { Beam 2 - ambient test of milled beam cross- } \\
\text { section with PT tendons }\end{array}$ \\
\hline C5 & $\begin{array}{l}\text { Costello } \\
(2013)\end{array}$ & Beam 1 - furnace test with PT tendons \\
\hline S6 & $\begin{array}{l}\text { Spellman } \\
(2012)\end{array}$ & Beam A - furnace test with PT tendons \\
\hline S7 & $\begin{array}{l}\text { Spellman } \\
(2012)\end{array}$ & Beam C - furnace test with PT tendons \\
\hline
\end{tabular}

\subsection{Software Selection}

A detailed selection process of the software used to perform the FEM modelling of the experiments was completed in order to ensure the most efficient attainment of the most accurate results. This selection is especially important and difficult for timber as few FEM software packages have built-in timber material capabilities. Additionally important for the modelling of PT timber beams in particular, is the compatibility of the material model with three-dimensional solid elements in order to capture the full mechanical behaviour, including shear and local deformation. The software packages considered for this project included Abaqus, ANSYS, LS DYNA, DIANA, SAFIR, and Vulcan. Abaqus is described in detail, followed by a brief introduction to the other considered software products.

\subsubsection{Abaqus}

Abaqus is a general purpose finite element suite which has many applications, the most relevant being thermal and mechanical simulations. Furthermore, the two analysis 
procedures may be performed in sequentially-coupled or fully-coupled analyses. The elements available for both processes include wire elements, shell elements and solid continuum elements. For heat transfer procedures involving discontinuities (such as phase changes with latent heat effects), first order diffusive elements should be used to implement the integration techniques required (Dassault Systèmes 2012). These solid elements are named DC3D8, in which DC denotes a heat transfer diffusion continuum element, 3D represents a three-dimensional element, and 8 is the number of nodes (linear elements have one node at each corner). More accurate quadratic elements are offered but should only be used in smooth, continuous simulations (Dassault Systèmes 2012). The diffusion elements allow for conduction properties to be defined as a function of temperature (isotropic, orthotropic or anisotropic), along with heat storage properties such as heat capacity and latent heat, and density. The user may implement an effective specific heat method or a latent heat method to account for the evaporation of moisture within the wood. However, the properties cannot be defined to be only one-way temperature dependent, so modelling in the cooling phase should be done with care.

Surfaces of solid elements are allowed to interact with fluids through means of contact properties, which may include convection and radiation to uniform or non-uniform heat sinks. Abaqus also allows the definition of cavity radiation, in which the view factors between each node in a defined cavity are computed and the internal surfaces are allowed to radiate between themselves. Additionally, a cavity approximation method is available which is less computationally demanding. An average-temperature radiation condition may be applied to the cavity surfaces in which an average surface temperature is computed, and subsequently radiative heat transfer is calculated to or from each node on the surface based 
on that average temperature. This procedure may be used if the cavity surfaces are of constant emissivity and are approximately isothermal, which the internal cavity in a wood box-beam typically is. The heat transfer analysis is done in Abaqus/Standard, an implicit finite element numerical solver.

In a mechanical analysis, an abundance of elements and solution procedures are available. In this case, as aforementioned, three-dimensional brick elements are of interest. Abaqus offers linear or quadratic elements which may employ a "reduced integration" scheme in which there are fewer integration points within the element. The possible elements for use are the C3D8, C3D8R, C3D20 and C3D20R, where C stands for continuum elements, 3D represents a three-dimensional element, 8 or 20 are the number of nodes, and $\mathrm{R}$ denotes reduced integration. For all of these elements, several built-in material models are available to describe the mechanical behaviour of various materials. Although there is no built-in model for timber behaviour, there are many available models which may lend themselves to wood (which is discussed in detail in Section 3.4.2). These include material models allowing for orthotropic property definition, asymmetric constitutive laws (within the concrete plasticity model), and the extended finite element method (XFEM) allowing for the use of linear-elastic fracture mechanics. Additionally, it is possible to use a user-defined material using subroutines written in the software's programming language, FORTRAN. User subroutines are outside the scope of this project.

A stress analysis may be done using Abaqus/Standard using an implicit finite element formulation, or Abaqus/Explicit for a more stable (albeit not necessarily accurate) analysis. Abaqus/Standard is preferable in order to ensure structural stability is guaranteed. The simulation may also include geometrical nonlinearity, and all mechanical properties may 
be temperature dependent. Abaqus/CAE is a graphical user interface which may be used to generate input files, and view or post-process results.

In addition to the available functionalities within Abaqus, software and support availability played a large role in the selection of this product. The University of Canterbury had existing access to the software and a research community with some past knowledge of its use. These vital factors lead to the selection of Abaqus as the FEM simulator for this research project.

\subsubsection{ANSYS}

ANSYS is also a general use FEM software with both thermal and stress analysis capabilities. It similarly allows for either fully-coupled or sequentially-coupled thermomechanical analyses, and allows for temperature dependent thermal properties (ANSYS Inc. 2015a)

The software allows for several material models for defining mechanical behaviour, including anisotropic elasticity and an anisotropic Hill's plasticity model which may be defined as functions of temperature. ANSYS also offers a generalized Hill's plasticity model which is also asymmetric in tension and compression. However, this model, which is perfectly suited for wood at ambient temperatures, is not allowed to be defined with temperature-dependent properties (ANSYS Inc. 2015b).

\subsubsection{LS DYNA}

LS DYNA is another general use FEM software which is primarily geared towards explicit dynamic analyses, but does allow for both linear and quasi-static analyses and thermal analyses, along with coupled multi-physics procedures. The software does not include a 
built-in wood material model, but does allow for user-defined materials. However, LS DYNA was not chosen because it is specialized in impact or highly dynamic procedures. (Livermore Software Technology Corporation, 2011)

\subsubsection{DIANA}

Another general purpose FEM software is DIANA, a Dutch product which began development in the earliest stages of computer programs in the 1970s (DIANA FEA 2017a). Today, DIANA has had an area focused on fire engineering applications and offers a 'staggered' sequentially-coupled thermo-mechanical analysis scheme (DIANA FEA 2017b). DIANA, like the other products, does not offer a material model specific to wood but offers orthotropic elasticity and Hill's orthotropic plasticity, which may be defined as functions of temperature.

\subsubsection{SAFIR}

SAFIR is a special purpose finite element software for structural fire applications developed at the University of Liege in Belgium (Franssen and Gernay 2017). The software may be used to perform sequentially-coupled thermo-mechanical analyses of various structural elements with temperature-dependent material properties. SAFIR automatically implements the latent heat method in order to account for the evaporation of moisture in wood (Franssen, 2005). SAFIR has recently included a wood-specific material model which employs the suggested parameters from Eurocode 5 Part 1-2 Annex B (thus only applicable in a standard fire) (CEN 2004b, Franssen and Gernay 2017). However, this material model is only available in two-dimensional beam elements with uniaxial stress laws, thus eliminating the possibility of orthotropic material modelling. Particularly with timber box-beams for post-tensioning, the anisotropic behaviour is highly important. 


\subsubsection{Vulcan}

Another special purpose structural fire engineering FEM software, developed at the University of Sheffield, is Vulcan. It was developed especially for the global structural fire analysis of composite steel framed building systems (Vulcan Solutions, n.d.). Threedimensional brick elements have recently been developed, however they do not offer a wood-specific material model (Burgess et al. 2016).

\subsection{Ambient Numerical Modelling}

It is ideal for a finite element model to work for both ambient and fire conditions. Many tests have been done at ambient conditions to simulate reduced cross-section behaviour that might result from fire exposure in wood structural elements. The aim of this study was to first create a model which is successful at ambient conditions to ensure wood mechanical behaviour is captured, and then extend that model to a thermally dependent simulation.

\subsubsection{Model Setup}

The beams were modelled with three-dimensional continuum elements (C3D8R), and the entire beams were modelled to capture any asymmetrical effects. The element type selection and mesh sensitivity study are discussed in Section 3.4.3. Steel plates were also modelled where required with solid continuum elements (C3D8R). The boundary condition and loading model choices are detailed in the following sections. Where applicable, the steel PT tendons were modelled with 3D, 2-node wire beam elements (B31). The analysis consisted of two or three steps total (depending on experiment type): an initial step where the constraints and boundary conditions were applied, a second step where the PT force was applied (if required) and a third step where the vertical loading was applied. Sample model assemblies are shown in Figure 3.1. 


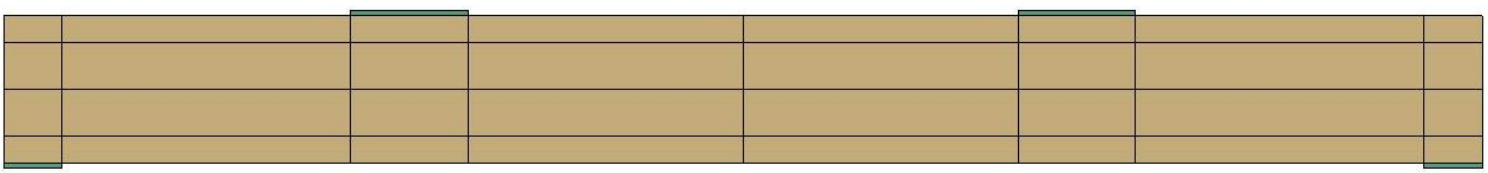

(a)

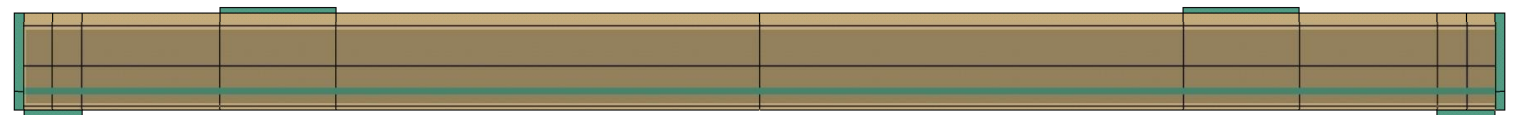

(b)

Figure 3.1: Sample model assembly (a) without PT tendon and (b) with PT tendon and anchorage plates (shown with view cut)

\subsubsection{Boundary Conditions}

The model boundary conditions (BC) may significantly influence the results and require particular attention. Several different iterations of modelling these conditions were done in order to investigate the effect of various scenarios and select the most realistic conditions. The support conditions were assumed to be simply supported, as the steel support plates were pinned to allow rotation in the experiments (Costello 2013, Spellman 2012). The span quoted in the modelled studies is assumed to be the centre-to-centre distance between supports for ambient tests and the clear span within the furnace for fire tests. The boundary conditions were modelled in several attempts. These condition variations are shown in Figure 3.2. Note that in the global coordinate system, the transverse axes are the X-axis (horizontal) and the $\mathrm{Y}$-axis (vertical), and the $\mathrm{Z}$-axis is the longitudinal axis.

Firstly, the boundary conditions were prescribed as line supports across the base of the beam, directly restraining the beam nodes (Figure 3.2a). Here, the vertical and lateral translation ( $\mathrm{Y}$ - and $\mathrm{X}$-axes, respectively), rotation about the vertical axis (Y-axis) and torsion about the longitudinal axis (Z-axis) were restrained at both ends, along with longitudinal translation (Z-axis) at one end of the beam. 

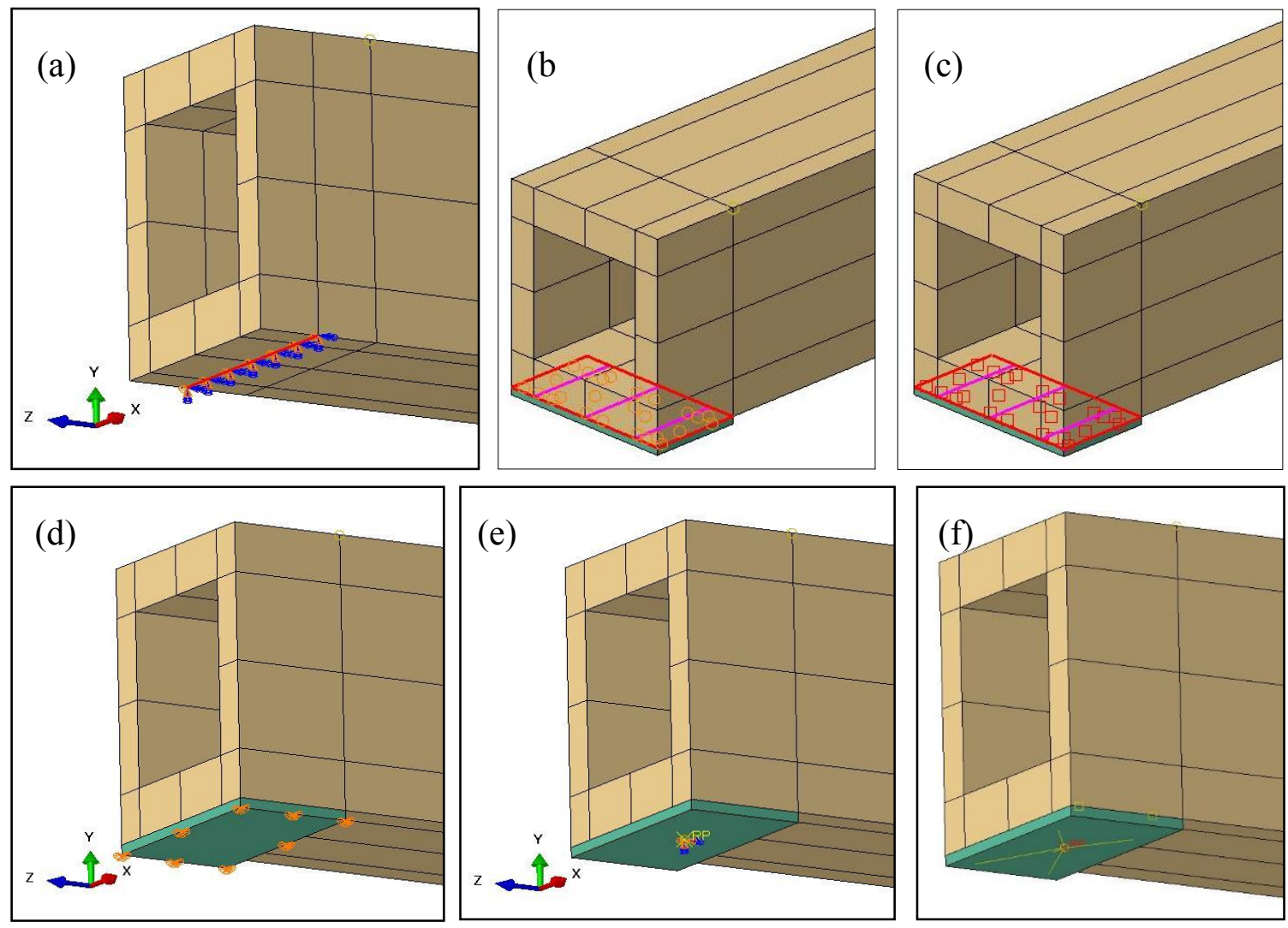

Figure 3.2: Various model boundary conditions: (a) line BC on beam nodes, (b) tied constraint between steel plate (master) and beam (slave), (c) surface-to-surface contact between steel plate (master) and beam (slave), (d) distributed BC on steel plate, (e) concentrated BC on reference point and (f) reference point coupling to steel plate

The support conditions were also modelled using interactions or constraints with $18 \mathrm{~mm}$ thick steel plates. Initially, a tied constraint was modelled between the top of the steel support plates and the bottom surface of the beam (Figure 3.2b). When modelled as a tied constraint to the beam surface, the boundary conditions were also prescribed as simply supported with the longitudinal translation (Z-axis) restrained on the steel plate only at one end.

Furthermore, the plates were modelled to interact with the bottom surface of the beam through surface-to-surface contact (Figure 3.2c). Using contact rather than a tie allows the nodes of the two parts to move independently of one another unless contact between the 
two surfaces is made, at which point a normal force will develop. This was defined as the default "Hard" contact in the normal behaviour contact property. Additionally, tangential behaviour was defined with a friction penalty of 0.4 . When modelled as a surface-tosurface contact, both plates were restrained from longitudinal translation (Z-axis) to ensure problem stability.

The restraint was either modelled as uniformly distributed over the steel support area (Figure 3.2d), or defined on a single reference point whose displacements and rotations was coupled to the rest of the steel plate surface nodes using uniform continuum distributing (Figure 3.2e and f). Over a distributed area, the bottom surface nodes of both plates were restrained in all translational degrees of freedom, thus inherently restraining the rotation of the steel plates. On the reference point, the node was restrained in all translational degrees of freedom and in the rotational degrees of freedom in the $\mathrm{Y}$ and $\mathrm{Z}$ axes. All boundary conditions and interactions were defined in the initial step created by Abaqus. The results for these various boundary conditions were compared to determine the most realistic situation. A comparison of the resulting mid-span deflections for the $\mathrm{BCs}$ is presented in Table 3.2 .

When a line of nodes on the beam are constrained, this results in stress concentrations which can induce small amounts of solution oscillation in the model due to the discontinuity, as can be seen in Figure $3.3 \mathrm{a}$ - note that the deformations have been magnified x5. 
Table 3.2: Comparison of deflection results for various support restraint and load conditions

\begin{tabular}{|c|c|c|}
\hline $\begin{array}{c}\text { Support } \\
\text { Condition } \\
\text { Nature }\end{array}$ & $\begin{array}{c}\text { Support } \\
\text { Connection to } \\
\text { Beam }\end{array}$ & $\begin{array}{c}\text { Mid-span } \\
\text { Deflection } \\
\text { (mm) }\end{array}$ \\
\hline Line & On beam nodes & 27.7 \\
\hline Distributed & Plate, tied & 20.5 \\
\hline Distributed & Plate, contact & 19.9 \\
\hline Reference Point & Plate, tied & 23.4 \\
\hline Reference Point & Plate, contact & 21.7 \\
\hline
\end{tabular}

The conditions with the supports modelled as a concentrated restraint on a reference point tied to the rest of the steel plate nodes had the closest deflection to experimental results. This is because the distributed plate support could not allow rotation as a pin or roller support condition would. The difference in the deformed shapes is shown in Figure 3.3b and c, where it may be observed that the distributed BC plate (b) did not rotate and resulted in more distortion in the elements directly above. Additionally, the plate with a surface-tosurface contact support provided more realistic results than the tied-surface constraint because the tie induced high tensile stresses from restricted uplift at the end face of the beam, shown in Figure 3.3d.

Thus, to represent the simply-supported beam support conditions, the reference point $\mathrm{BC}$ was used in combination with the contact interaction between the steel plate and beam surface. For model C5 (Costello's furnace test), the distributed BC was used on the steel plate rather than the reference point. This provided a better representation of the true experiment behaviour as the beam rested directly on the furnace concrete blocks, restraining the rotation of the support. In order to ensure stability in analyses with posttensioning wires, additional boundary conditions directly on the beam part were required. 
In these scenarios, a line boundary condition was defined on each end of the beam over the center of the supports restraining lateral and vertical movement, and longitudinal movement on one end. This was required because the PT force is applied in the first step before any vertical load is applied. The implication of this is that there is no normal force between the beam and the steel support, indicating that there is also no lateral force of friction restraining the beam. This renders the simulation unstable unless lateral restraints are added to the beam itself. The interaction between the restrained steel plate supports and the beam stabilized the convergence of these $\mathrm{BC}$ 's and no oscillation was seen in the solution.
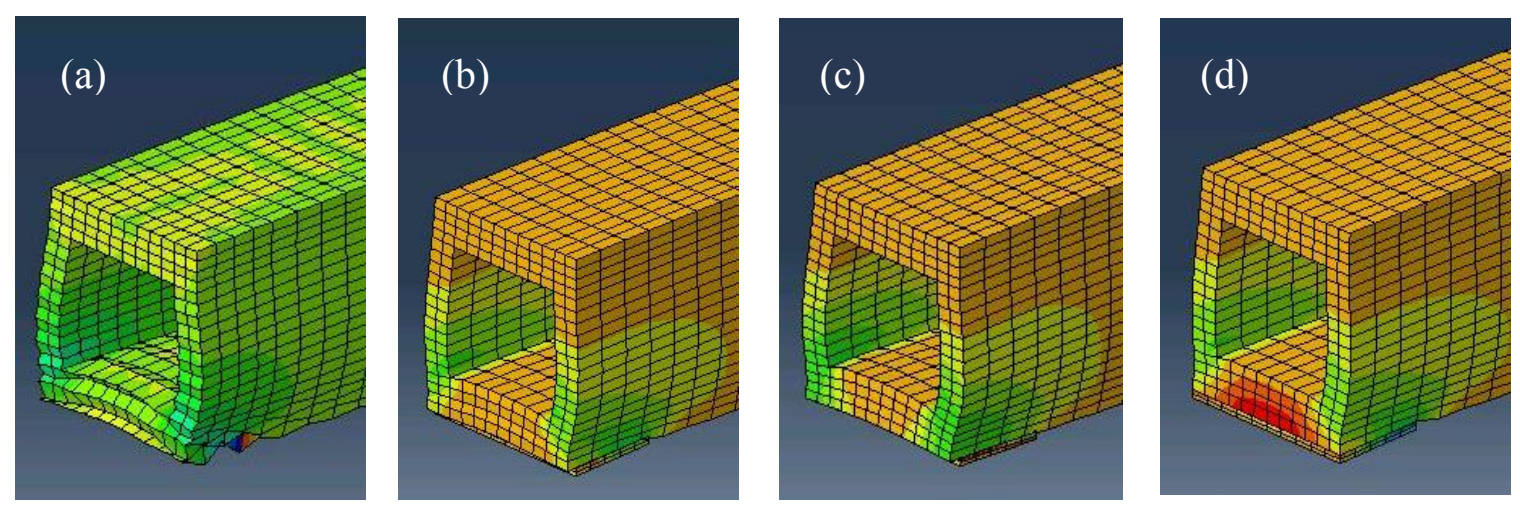

Figure 3.3: Comparison of contour results for stress in the vertical axis (S33) on deformed shapes (magnified x5) for (a) line BC on beam nodes, (b) distributed BC with surface-to-surface contact, (c) reference point $B C$ with surface-to-surface contact and (d) distributed $B C$ with tied constraint

Where post-tensioning was involved, the end nodes of the tendon wire were tied as slave nodes to the surface of steel anchorage plates at each end of the beam using node-to-surface discretization method (Figure 3.4a). The anchorage plates were tied to the end surfaces of the beam (where the anchorage plate was the master surface), using the surface-to-surface discretization method (Figure 3.4b). 

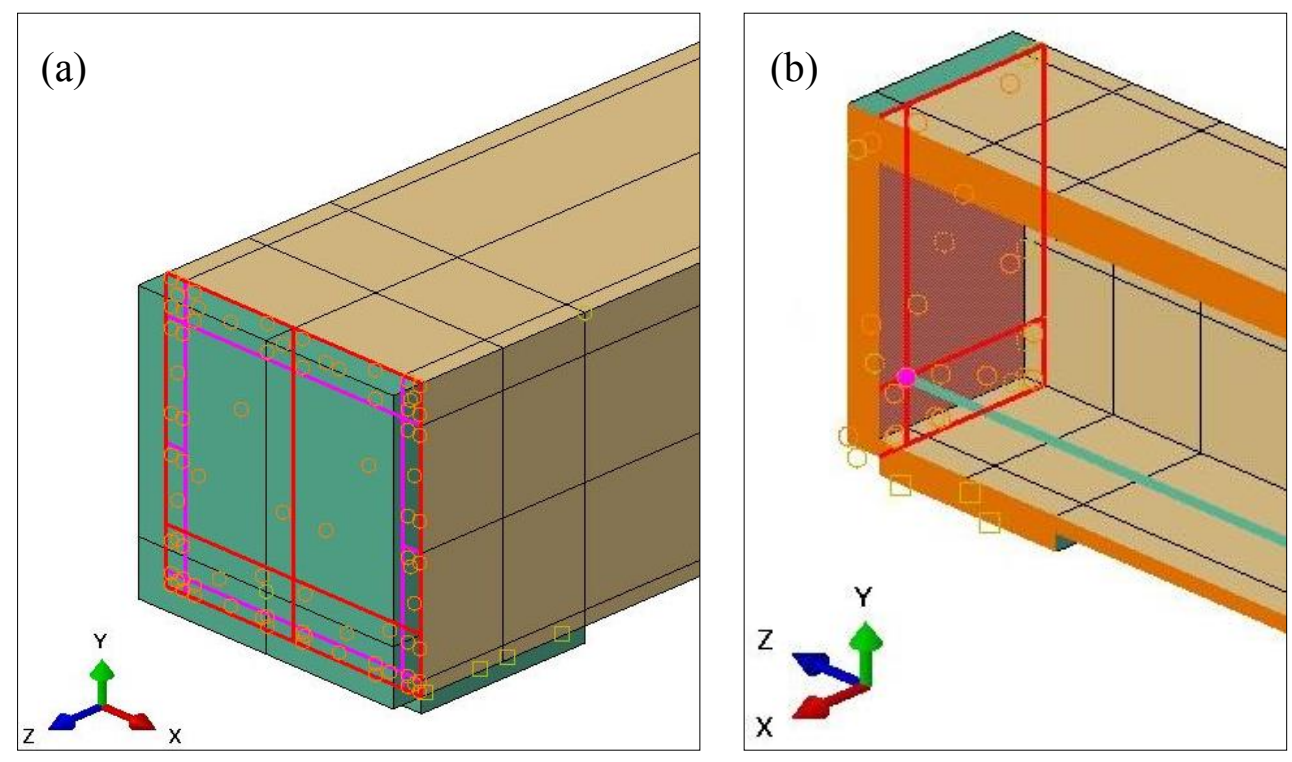

Figure 3.4: PT system model: (a) tied constraint between steel anchorage plate (master) and beam end (slave) and (b) tied constraint between steel anchorage plate (master) and steel tendon end node (slave)

\subsubsection{Loading}

The vertical loading was modelled using steel loading plates which were tied as master surfaces to the top surface of the beam (the slave), using the surface-to-surface discretization method (shown in Figure 3.5a). The load was applied linearly ramped over the step as a pressure over the surface of the two steel plates, defined by the total force at failure in each respective experiment (shown in Figure 3.5b). Additionally, loads beyond the failure load or a displacement controlled loading method were applied at times to investigate the model behaviour. For displacement controlled loading, reference points were defined in the center of the top surfaces of the loading plates which were coupled to the rest of their respective surfaces using continuum distributing on all degrees of freedom (see Figure 3.5c). A displacement boundary condition was then applied to these reference points in the loading step with an imposed vertical translation rather than an applied load (shown in Figure 3.5d). 

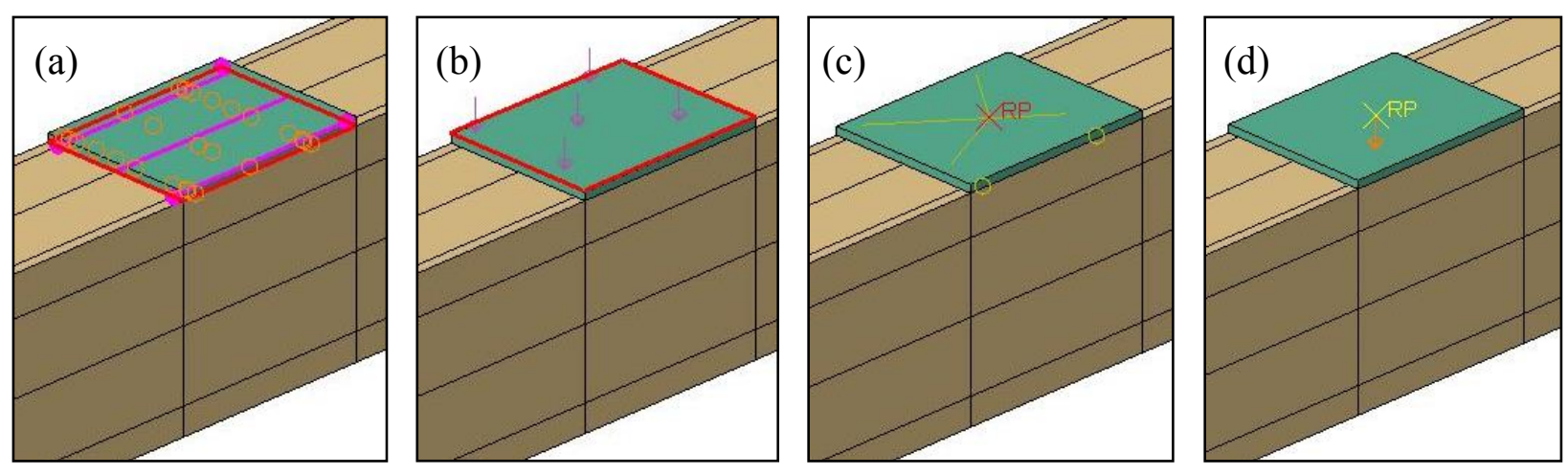

Figure 3.5: Load application in model by steel loading plate showing (a) steel plate (master) tied to beam top surface (slave), (b) pressure load applied to top loading plate surface, (c) reference point coupled to the loading plate surface and (d) displacement imposed on reference point

Where applicable, the PT force was modelled as a bolt load on the tendon. The bolt load is available in Abaqus for pre-tensioning bolts, but may also be extended to PT strands (Van Beerschoten 2013). The beam part was partitioned into three segments, the middle element being $100 \mathrm{~mm}$ long. The bolt load was applied to this middle segment using the "Apply force" method (shown in Figure 3.6). The total PT force was applied to the central segment of the tendon in the first general step, which linearly ramped up the tensile force in the tendon by shortening the segment until the defined PT force was attained. In the subsequent vertical loading step, the bolt load method was modified to "Fix at current length". This allows the initial length change from the post-tensioning to be maintained, while the stress in the tendon may change throughout the step due to tendon elongation. 


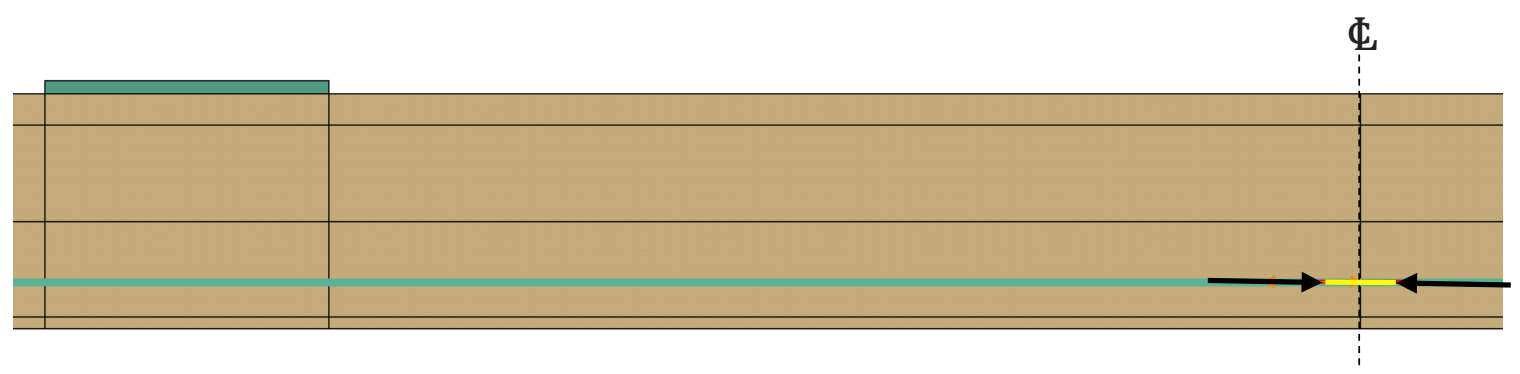

Figure 3.6: Section of beam assembly showing where bolt load is applied to tendon central partition (highlighted segment of wire), shortening the partition until the specified force is achieved while placing the rest of the tendon in tension

\subsubsection{Material Models}

Steel is simple to model in Abaqus as the ideal constitutive model is readily characterized by the pre-defined metal material definitions within the software. Wood is a challenge to model in Abaqus in order to include the anisotropy and natural defects in the material, along with an asymmetric constitutive model in tension and compression. Additionally, wood damage models should realistically include crack initiation. Herein, model C1 is discussed as an example.

\subsubsection{Steel Elements}

For the steel plates and tendons, an elastic isotropic material model was employed with a Young's modulus of $200 \mathrm{GPa}$ and a Poisson's ratio of 0.3 (Van Beerschoten 2013, Costello 2013). In all cases, steel plates and high-strength tendons were loaded within their linear elastic range, so no material model for plasticity was required.

\subsubsection{Wood Elasticity}

As a first step, a model with isotropic elasticity was created in order to observe the effects of ignoring wood's isotropy. The manufacturer specified value for the modulus of elasticity of $13200 \mathrm{MPa}$ was used, with a typical Poisson's ratio of 0.4 . This initial test resulted in 
behaviour which was much too stiff, as may be observed in Figure 3.7, where all simulations were deflection controlled. This confirms that wood behaviour is highly influenced by its reduced stiffness (and strength) perpendicular to the grain.

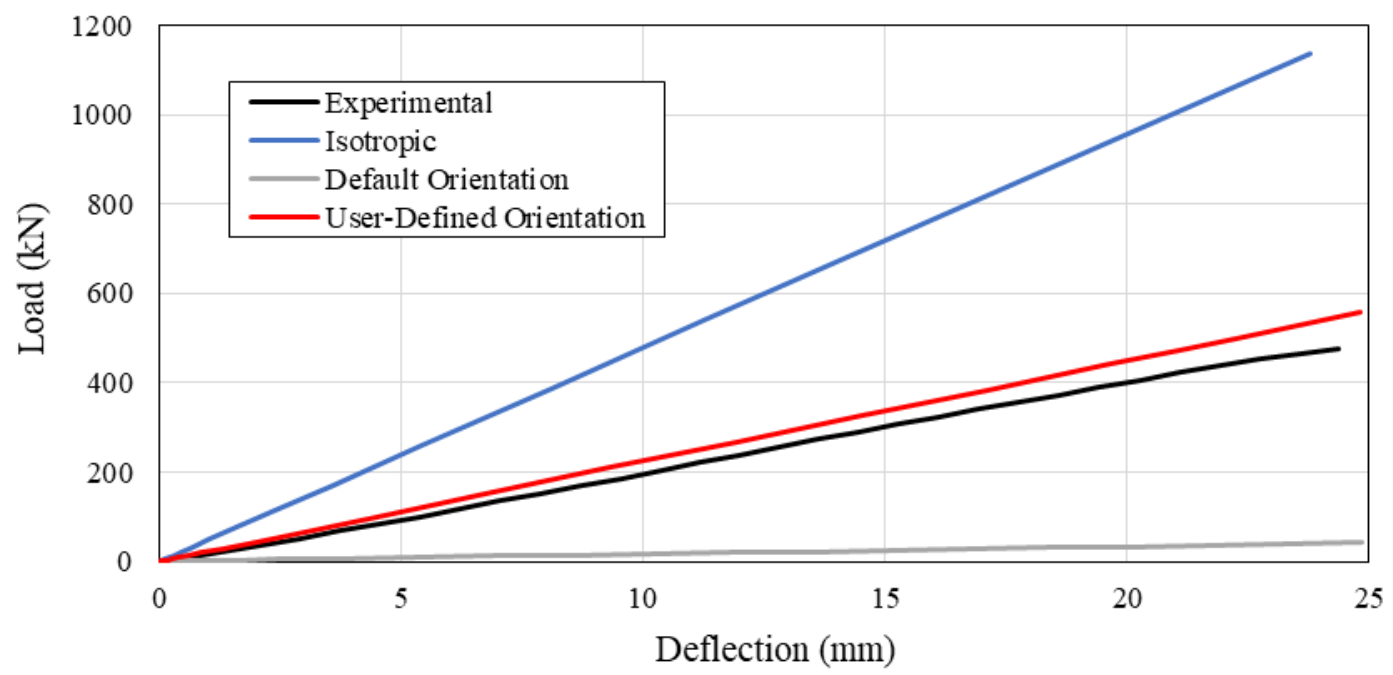

Figure 3.7: Load versus deflection plot for simulations of beams using various linear elastic material models, with displacement controlled loading, compared to experimental results

Thus, an orthotropic linear elastic model using Engineering Constants was employed. This allows the material properties to be fully defined by nine constants including the three orthotropic elastic moduli, three Poisson's ratios and three shear moduli. One limitation of this model is that it cannot distinguish between the stiffness in tension and compression, which will become a problem when combined with a thermal analysis. This issue arises from the definition in Eurocode 5, Part 1-2, Annex B of mechanical properties of wood as a function of temperature, as the stiffness is modified by different factors in tension and compression (CEN 2004b).

Mechanical properties suggested by Van Beerschoten (2013) after experimental testing of radiata pine LVL were adapted with fairly successful results. The material properties 
adapted from Van Beerschoten's experimental values recommended for FEM analysis (rounded for more general results due to differences in wood product used) were given in Table 3.3. In order for the model to be applicable to LVL and Glulam, the transverse directions were assumed to be equal as both may roughly be represented as transversely isotropic (Glulam does not have obvious tangential and radial material directions, as demonstrated in Figure 2.1a).

While using the engineering constants model, the importance of the material orientation is clear. The Abaqus/CAE module was used to define the beam part with 3D brick elements extruded along the beam axis. In the default global coordinate system, the primary X-axis is oriented in the horizontal transverse direction compared to the extrusion. With the engineering constants defined in this default global coordinate system, the resulting behaviour was more flexible than the experimental beam, seen in Figure 3.7. This is likely due to incompatibility with the $3 \mathrm{D}$ element stiffness formulation and the direction of extrusion using the graphical user interface. However, defining a new coordinate system with the primary $\mathrm{X}$-axis as the longitudinal beam direction and defining the constants as such (for the exact same material properties), results in a much more accurate stiffness (as shown in Figure 3.7). The adapted material properties used to demonstrate the effect of material orientation definition on the results are presented in Table 3.3. The same Poisson's ratios were also maintained, using Equation 3.1 to convert between orientation systems.

$$
\frac{v_{i j}}{E_{i}}=\frac{v_{j i}}{E_{j}}
$$

Equation 3.1 
Table 3.3: Comparison between different material orientation definitions for the same mechanical properties

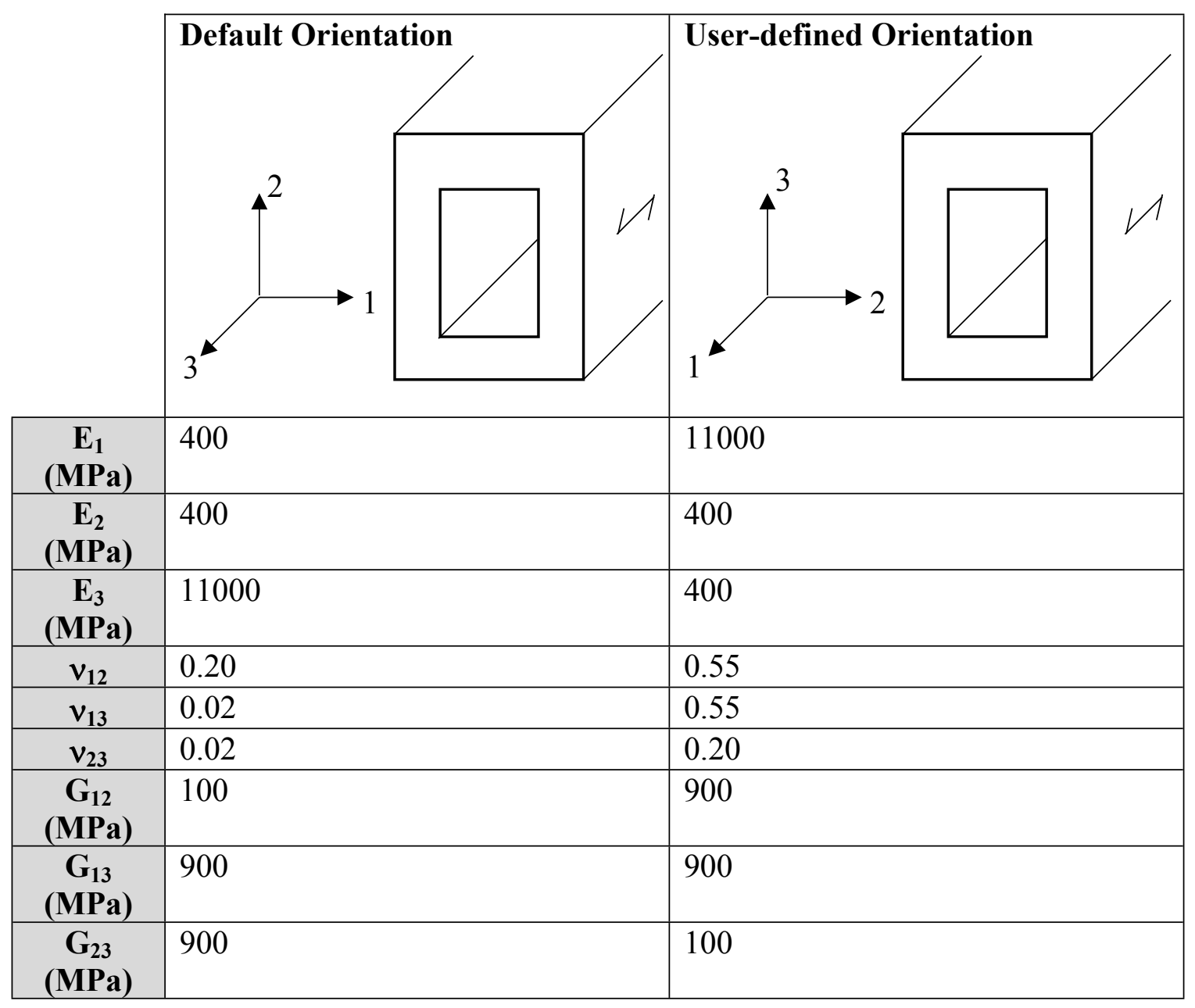

In order to calibrate the material properties to those for Costello's tests, which used a slightly different class of LVL, the manufacturer recommended values that were available were used for the longitudinal modulus of elasticity and shear modulus of $13200 \mathrm{MPa}$ and $660 \mathrm{MPa}$, respectively. Due to a lack of recommended values, the same rounded moduli of $400 \mathrm{MPa}$ (Young's), $100 \mathrm{MPa}$ (shear) were used for the transverse properties of the material. Thus, the final values used for the engineering constants elasticity model are presented in Table 3.4, with a user-defined material orientation with the primary axis along the longitudinal beam direction. 
Table 3.4: Input parameters for engineering constants orthotropic elasticity

\begin{tabular}{|l|l|l|l|l|l|l|l|l|}
\hline $\begin{array}{l}\text { E1 } \\
\text { (MPa) }\end{array}$ & $\begin{array}{l}\text { E2 } \\
\text { (MPa) }\end{array}$ & $\begin{array}{l}\text { E3 } \\
\text { (MPa) }\end{array}$ & $v 12$ & $v 13$ & $v 23$ & $\begin{array}{l}\text { G12 } \\
\text { (MPa) }\end{array}$ & $\begin{array}{l}\text { G13 } \\
\text { (MPa) }\end{array}$ & $\begin{array}{l}\text { G23 } \\
\text { (MPa) }\end{array}$ \\
\hline 13200 & 400 & 400 & 0.55 & 0.55 & 0.2 & 660 & 660 & 100 \\
\hline
\end{tabular}

\subsubsection{Wood Plasticity}

In order to account for additional nonlinear behaviour, a plasticity or damage model should be introduced. A number of available models were tested and compared to determine which could best handle the complexities of wood behaviour. The following paragraph gives a brief introduction to the Abaqus material models that were considered as candidates to represent wood nonlinear behaviour.

The "Concrete Damage Plasticity" (CDP) model has been used in the past to approximate wood behaviour due to the availability of an asymmetric constitutive model. In this respect, wood may perform in a similar way to concrete with elasto-plastic behaviour in compression and elasto-brittle behaviour in tension. However, the model requires the properties to be defined isotropically, including the elastic properties. Another pre-built material model in Abaqus is the Hill's Potential Function for plasticity which allows for the definition of orthotropic yield criteria. This model does not, however, allow for an asymmetric constitutive model.

Another material model that was considered was the "Damage for Fiber-Reinforced Composites", due to the similarities in material structure to wood, which consists of a series of parallel fibers bonded with the naturally occurring adhesive, lignin.

Several methods were also considered to model the cracking behaviour of wood. Cohesive elements may be used to model crack propagation along a pre-defined path, which can be 
useful in modelling adhesive lines and crack formation in wood where cracks primarily open specifically along the wood grain direction. Abaqus also incorporates linear-elastic fracture mechanics into the "Damage for Traction Separation Laws" material models using the extended finite element method (XFEM). Abaqus also offers the option of including a user-defined material using a subroutine, however the intent of this research is to use and evaluate the existing models available. Table 3.5 gives a comparison of all the material models considered for use within Abaqus without necessitating the use of a user-defined material and subroutine.

Of the available models, three were chosen as the most promising to create comparison models. These three were the Hill's Potential Function for orthotropic plasticity, the Concrete Damaged Plasticity model for asymmetric constitutive models, and the XFEM crack propagation model for linear-elastic fracture mechanics. The benefits and drawbacks of each model are discussed in the following sections. 
Table 3.5: Summary of a preliminary comparison between different model options for non-linear material modelling of wood

\begin{tabular}{|c|c|c|c|}
\hline $\begin{array}{l}\text { Plasticity } \\
\text { Model }\end{array}$ & Pros & Cons & Notes \\
\hline \multirow{3}{*}{$\begin{array}{l}\text { Concrete } \\
\text { Damage } \\
\text { Plasticity }\end{array}$} & $\begin{array}{l}\text { Asymmetrical } \\
\text { constitutive model } \\
\text { definition for } \\
\text { different compressive } \\
\text { and tensile behaviour }\end{array}$ & $\begin{array}{l}\text { Requires that the elastic } \\
\text { behaviour of the } \\
\text { material be isotropic } \\
\text { and linear }\end{array}$ & $\begin{array}{l}\text { Field variables may be } \\
\text { defined as functions of } \\
\text { material directions, but the } \\
\text { use of these require a user } \\
\text { subroutine USDFLD }\end{array}$ \\
\hline & & $\begin{array}{l}\text { Plasticity and damage } \\
\text { definitions are isotropic }\end{array}$ & \\
\hline & & $\begin{array}{l}\text { Does not capture shear } \\
\text { yielding }\end{array}$ & \\
\hline \multirow{2}{*}{$\begin{array}{l}\text { Plasticity } \\
\text { with Hill's } \\
\text { Potential } \\
\text { Function }\end{array}$} & $\begin{array}{l}\text { Orthotropic yield } \\
\text { criteria }\end{array}$ & $\begin{array}{l}\text { Assumes the same } \\
\text { behaviour in tension } \\
\text { and compression }\end{array}$ & \\
\hline & & $\begin{array}{l}\text { Shear strength of wood } \\
\text { does not follow the } \\
\text { assumption } \tau=\sigma / \sqrt{3} \text {, } \\
\text { so the formulation } \\
\text { might not be accurate }\end{array}$ & $\begin{array}{l}\text { The actual shear strength in } \\
\text { each material direction may } \\
\text { be defined as a fraction of } \\
\text { this } \tau \text { value }\end{array}$ \\
\hline $\begin{array}{l}\text { Fibre- } \\
\text { Reinforced } \\
\text { Composite } \\
\text { Damage }\end{array}$ & $\begin{array}{l}\text { Allows for crack } \\
\text { formulation parallel } \\
\text { to the grain }\end{array}$ & $\begin{array}{l}\text { May only be used with } \\
\text { shell elements }\end{array}$ & $\begin{array}{l}\text { Shell elements may be } \\
\text { applicable for wood veneers } \\
\text { or plane-type elements, } \\
\text { however they are not ideal } \\
\text { for Glulam or heat transfer } \\
\text { analyses }\end{array}$ \\
\hline $\begin{array}{l}\text { Cohesive } \\
\text { Elements }\end{array}$ & $\begin{array}{l}\text { Allows for crack } \\
\text { propagation along } \\
\text { adhesive lines with } \\
\text { traction versus } \\
\text { separation for tension } \\
\text { and shear }\end{array}$ & $\begin{array}{l}\text { Will only crack along a } \\
\text { user-defined path }\end{array}$ & $\begin{array}{l}\text { Typically in tests cracks } \\
\text { developed through the wood, } \\
\text { not necessarily along } \\
\text { adhesive lines }\end{array}$ \\
\hline \multirow[t]{2}{*}{ XFEM } & $\begin{array}{l}\text { May use traction- } \\
\text { separation law } \\
\text { damage models to } \\
\text { employ linear-elastic } \\
\text { fracture mechanics } \\
\text { (LEFM) }\end{array}$ & $\begin{array}{l}\text { Crack propagation } \\
\text { direction cannot be } \\
\text { defined as along the } \\
\text { wood grain }\end{array}$ & $\begin{array}{l}\text { LEFM is valid only with } \\
\text { small-scale yielding }\end{array}$ \\
\hline & $\begin{array}{l}\text { No initial defect or } \\
\text { defined crack plane is } \\
\text { required, simply a } \\
\text { crack nucleation } \\
\text { criterion }\end{array}$ & & \\
\hline
\end{tabular}




\section{Hill's Potential Function}

The Hill's Potential Function was chosen as a potential candidate as it can represent the variable strengths in each orthotropic direction, similar to the Engineering Constants elastic model. The model can be defined as a sub-option for the "Plastic" material behaviour model called "Potential". The strengths in each direction are defined as a fraction of a reference yield strength and corresponding shear strength. The reference shear strength is assumed by the model to be the reference yield strength divided by the square-root of three, i.e. $\tau_{0}=\sigma_{0} / \sqrt{3}$. Subsequently, the yield strengths in all directions are defined as $R_{i i}=$ $f_{i i} / \sigma_{0}$ and the shear strengths are defined as $R_{i j}=f_{i j} / \tau_{0}$. One negative aspect about this model is that the constitutive model is assumed to be symmetric, and cannot distinguish between strengths in tension and compression. This can be easily dealt with at ambient because the failure of tension and compression often do not occur simultaneously, so the strength value corresponding to the first failure mode may be used for reasonably accurate results. It may, however, become more limiting (in the same way as the Engineering Constants elasticity model) when coupled with a thermal analysis due to the dependency of the thermally modified strength properties on tensile versus compression stresses.

As a comparative example, Model C1 was defined with the Engineering Constants model (with properties as defined in Table 3.4 for elasticity) adding the Hill's Potential Function for plasticity. When analyzed elastically, the maximum tensile stress in the beam was below the manufacturer tensile strength of $33 \mathrm{MPa}$, and the maximum compression stress was below the manufacturer compression strength of $38 \mathrm{MPa}$, but above $33 \mathrm{MPa}$. As a result, the reference stress $\sigma_{0}$ was taken as $38 \mathrm{MPa}$, and the longitudinal strength was taken as $100 \%$ of the reference value. At ambient, it does not have an effect on this test because 
either way, the tensile stresses did not reach the yield point. The strength in the transverse direction was taken as $10 \mathrm{MPa}$, which is the manufacturer strength for compression perpendicular to grain. Additionally, the shear stresses from the elastic analysis were above the manufacturer shear strength of 5.3 MPa. This value was used as the shear strength in all directions. A summary of the strengths and strength ratios used in the model are given in Table 3.6. The orientation used is the user-defined orientation from Table 3.3.

Table 3.6: Strength properties used in Hill's Potential Function plasticity model

\begin{tabular}{|l|l|l|l|l|l|}
\hline $\boldsymbol{\sigma}_{\mathbf{0}}$ & $38 \mathrm{MPa}$ & $\boldsymbol{R}_{\boldsymbol{i i}}$ & $\boldsymbol{\tau}_{\mathbf{0}}$ & $38 / \sqrt{3}=21.9 \mathrm{MPa}$ & $\boldsymbol{R}_{\boldsymbol{i} \boldsymbol{j}}$ \\
\hline $\boldsymbol{f}_{\mathbf{1 1}}$ & $38 \mathrm{MPa}$ & 1.0 & $\boldsymbol{f}_{\mathbf{1 2}}$ & $5.3 \mathrm{MPa}$ & 0.242 \\
\hline $\boldsymbol{f}_{\mathbf{2 2}}$ & $10 \mathrm{MPa}$ & 0.263 & $\boldsymbol{f}_{\mathbf{1 3}}$ & $5.3 \mathrm{MPa}$ & 0.242 \\
\hline $\boldsymbol{f}_{\mathbf{3 3}}$ & $10 \mathrm{MPa}$ & 0.263 & $\boldsymbol{f}_{\mathbf{2 3}}$ & $5.3 \mathrm{MPa}$ & 0.242 \\
\hline
\end{tabular}

A simulation applying this material to model $\mathrm{C} 1$ resulted in failure runaway deflections at the failure load, compared to a maximum deflection of $21.0 \mathrm{~mm}$ for just a purely elastic Engineering Constants model. However, the maximum shear stress in the shear zone between the support and the loading plate is limited to $5.3 \mathrm{MPa}$ as that was the yield strength used in the model, compared to the experimentally measured shear stress of 6.9 MPa. This indicates that the strength of the wood is actually higher than the manufacturer specified strength, which is typically expected. In a design scenario, using the manufacturer strength would be a conservative approach as it can over-predict the beam deflection.

As employed above with one yield strength for each material direction, the constitutive model consists of a linear elastic portion followed by a yield plateau. This is not a realistic constitutive model for timber and could be improved upon, especially with an asymmetric constitutive model allowing for brittle failure in tension. However, with the limitations 
imposed on this material model, varying the constitutive model to more complex relationships had little effect on the resulting beam behaviour, and the elasto-plastic behaviour is an acceptable approximation for compression (Buchanan and Abu 2017). Therefore, the bilinear constitutive model presented in Figure 3.8 was used for all of the Hill's plasticity models.

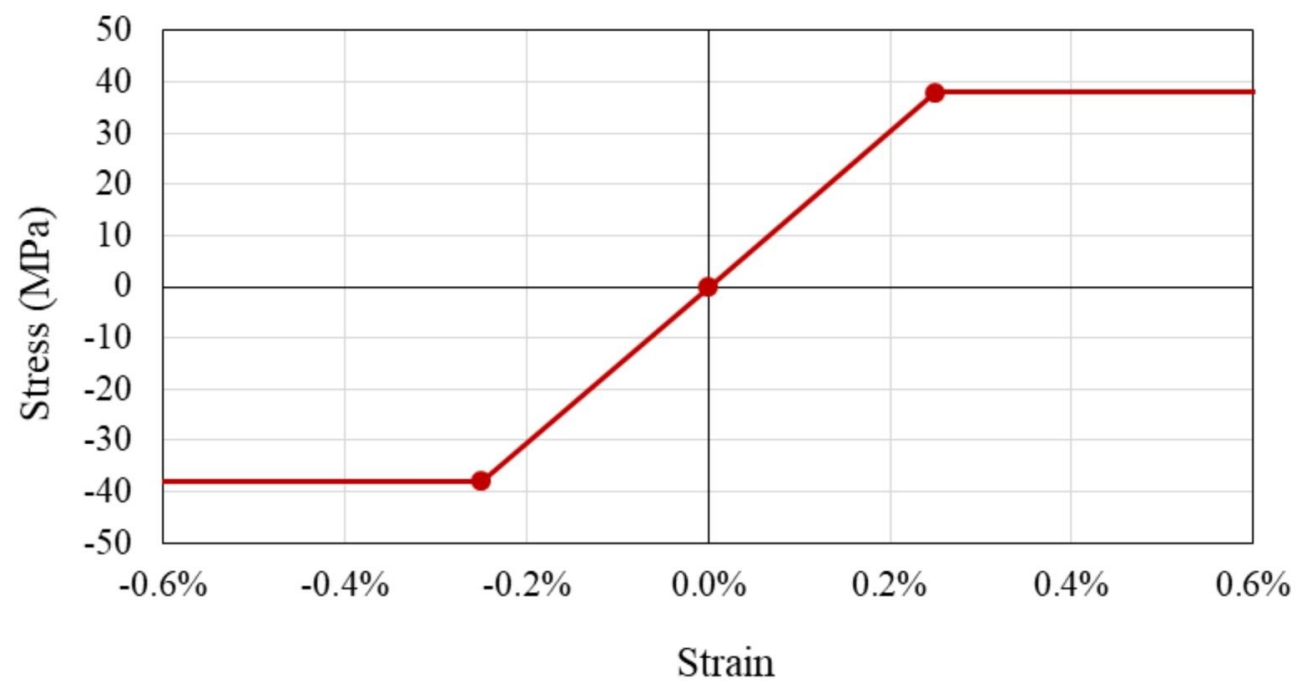

Figure 3.8: Bilinear symmetrical constitutive model used in ambient numerical models due to material definition constraints

\section{Concrete Damaged Plasticity}

Another built-in material behaviour model which could be useful in modelling timber is the Concrete Damaged Plasticity (CDP) model. It has been used several times in conjunction with thermal analyses with fairly successful results (i.e. Menis 2012). The biggest benefit of using the CDP is that it allows the user to define different material behaviour in compression and tension. However, there are several drawbacks to the model, especially when used for solely ambient analysis. Firstly, the CDP does not allow for orthotropic material properties. The plasticity model itself does not allow for anisotropic property definition, and the software does not allow the model to be paired with any 
elasticity model other than isotropic elasticity. As was discussed in Section 3.4.2.2, the isotropic elastic material model is much too rigid and results in highly under-predicted deflections.

The CDP model requires several input parameters that relate specifically to concrete which makes it difficult to define the values to be used for wood. For example, some of the main required parameters include the dilation angle and eccentricity for the Drucker-Prager yield surface (discussed below), and the ratio of the biaxial compressive strength to the uniaxial yield strength. These parameters are typically used to describe theoretical plastic behaviour of concrete, but the values for the parameters themselves are difficult to determine, even for concrete. There are some default values recommended by Abaqus, but they do not necessarily remain true when dealing with wood.

In order to clarify what these parameters represent, Figure 3.9 shows a graphical representation of the dilation angle and eccentricity related to the hyperbolic DruckerPrager yield and plastic flow model. If the Drucker-Prager function was plotted for every axis $q$ in the deviatoric plane - that is, revolved around the $p$ axis, the familiar cone-shaped yield surface would be generated. As can be seen in Figure 3.9, the dilation angle as defined in Abaqus's CDP model is the rate at which the yield surface grows with hydrostatic (confinement) stress $p$ (which may be equal to the friction angle), and the eccentricity relates to the rate at which the hyperbolic function asymptotically approaches the linear function. These properties are also related to another required parameter, ratio of biaxial compressive strength to uniaxial compressive strength, and are related to concrete performance but not wood performance. It is therefore difficult to defend values of these 
parameters used for wood. Values for the parameters used in previous studies which utilized the CDP in Abaqus were not readily available in the published literature.

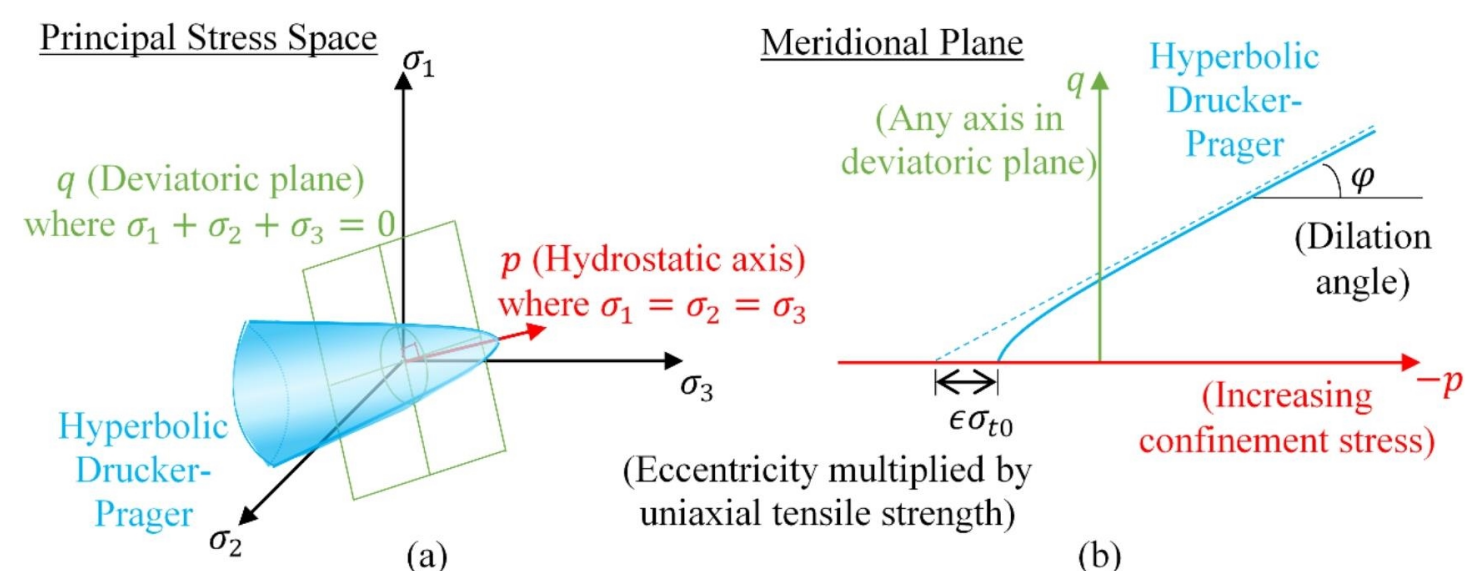

Figure 3.9: Representation of required parameters for CDP showing (a) the hydrostatic axis and deviatoric stress plane in the principal stress space and (b) the Drucker-Prager function in a meridional plane as defined by the dilation angle and eccentricity

The constitutive model and failure stresses can be defined independently and can thus approximate the typical timber constitutive model more accurately. The constitutive model input is shown in Figure 3.10. This relationship was modified from the constitutive model defined by Van Beerschoten (2013) after experiments described in Section 2.2.1.1. 


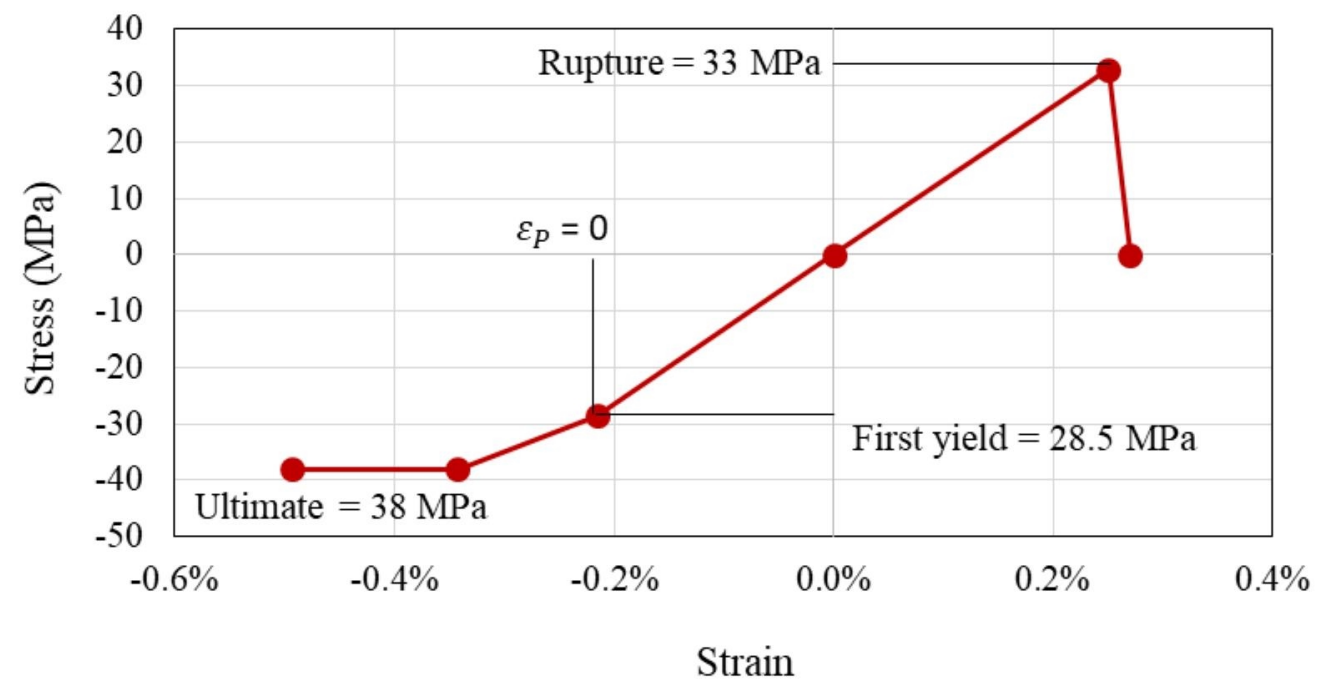

Figure 3.10: Constitutive model implemented for the strength in CDP material definition The model does not allow you to directly specify shear behaviour, which was the primary mode of failure for all of Costello's tests (2012) and is often the case in timber box-beams. For example, in model $\mathrm{C} 1$, the compressive and tensile yield strengths were never reached in the analysis. When run with the CDP, the model reached shear stress values much higher than the shear strength (up to 11.5 MPa). Furthermore, there was no additional mid-span deflection at the failure load when compared to the purely isotropic elastic case, which was $8.55 \mathrm{~mm}$ (compared to $24 \mathrm{~mm}$ experimentally). Due to this fact, it was also not possible to investigate the model sensitivity to the aforementioned input parameters using Costello's ambient beam tests because the introduction of the CDP material model had no influence on the deflection results (discussed in Section 3.4.4.1). For the limited results shown herein, the input parameters outlined in Table 3.7 were used.

Table 3.7: Concrete Damaged Plasticity material model input parameters

\begin{tabular}{|c|c|c|c|c|}
\hline Dilation Angle & Eccentricity & $\boldsymbol{f}_{\boldsymbol{b} \mathbf{0}} \boldsymbol{f}_{\boldsymbol{c} \mathbf{0}}$ & $\mathbf{K}$ & $\begin{array}{c}\text { Viscosity } \\
\text { Parameter }\end{array}$ \\
\hline $30^{\circ}$ & 0.1 & 1 & 0.67 & 0.0001 \\
\hline
\end{tabular}




\section{XFEM}

The extended finite element method (XFEM) utilizes an enriched domain in the numerical formulation in order to allow cracks to propagate through elements without requiring remeshing. In order to implement linear-elastic fracture mechanics (LEFM) into the model, the XFEM is based on the partition of unity method and the virtual crack closure technique (VCCT) (Xiea and Biggers 2006). A damage initiation condition may be defined in the material model called the "fracture criterion", which Abaqus will search for in the enriched domain throughout the simulation. Once this fracture criterion has been met at a given element (within a user-defined tolerance), a crack will nucleate in that element. For this method, the fracture criterion may be based on either a maximum principal stress or strain. Crack evolution is defined in the material model and in a specialized contact interaction property. A crack must propagate through an entire element instantly (i.e. there cannot be a crack tip in the middle of an element), and will propagate at solution-dependent direction and rate. By using this method, it is not necessary to define any initial defect or crack initiation points, or a pre-defined crack path.

After a preliminary trial a crack had nucleated at a reasonable location (see Figure 3.11) when compared to experiment crack development, and the results for a principal stress fracture criterion appeared encouraging. It was expected that upon further exploration, the crack would propagate along the length of the model in the shear region, allowing for increased deflection and limiting the development of shear stresses. 
(a) Scalar Damage Variable

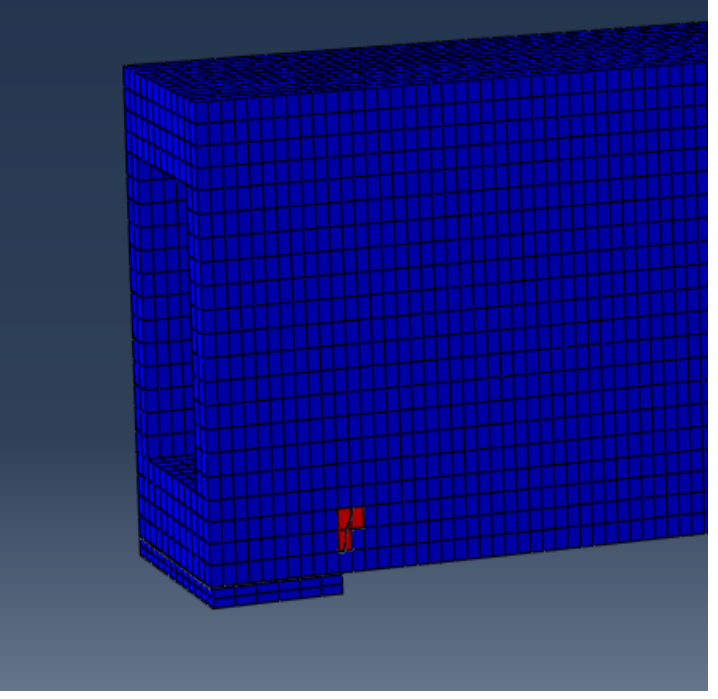

(b) Principal Stress

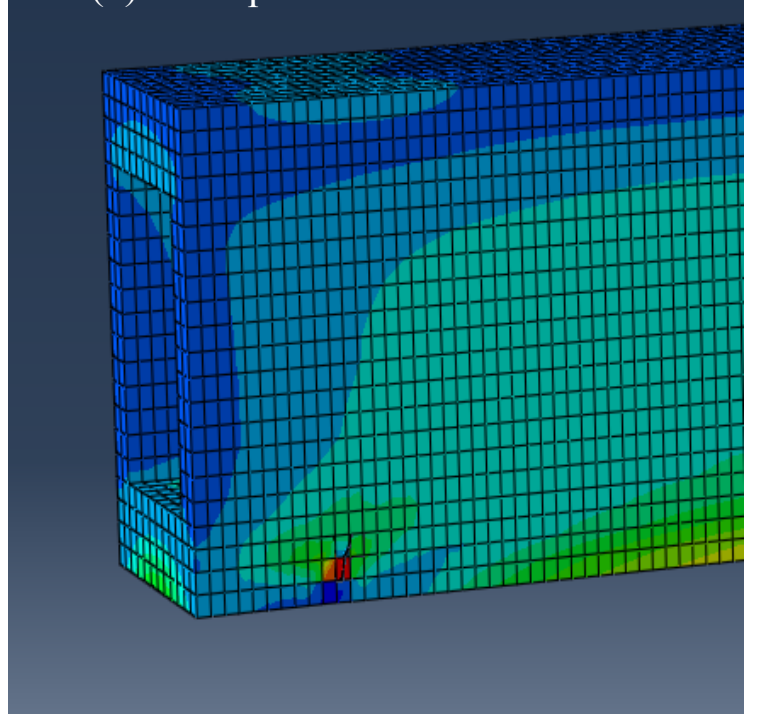

Figure 3.11: Sample crack development in quick XFEM trial (a) from maximum principal stress (b)

However, the results achieved did not exhibit the expected behaviour. An attempt was made to implement the XFEM model in several different ways. The model with the best success was implemented using the "maxps" (maximum principal stress) damage initiation criteria. This was defined as a material behaviour for the wood along with the engineering constants elasticity model. The only input parameter required for the "maxps" model is the maximum allowable principal stress at which crack nucleation occurs. This was difficult to define due to the anisotropy of wood, especially at an unknown angle to grain. However, because it was a four point loading test, and cracks were typically generated in the shear region, it was assumed that the stress state at crack nucleation would be a pure shear stress state. According to plane-stress theory (Equation 3.2 and Equation 3.3), with pure shear stress the principal angle is $45^{\circ}$ (see Figure 3.12). In Equation 3.2 below, $\theta_{p}$ is the principal angle, $\sigma_{x}$ and $\sigma_{y}$ are the element normal stress states, and $\tau_{x y}$ is the element shear stress state. 


$$
\tan 2 \theta_{p}=\frac{2 \tau_{x y}}{\sigma_{x}-\sigma_{y}}
$$

Equation 3.2

For no normal stresses, the fraction denominator will go to zero and the fraction goes to infinity, thus:

$$
\theta_{p}=\frac{1}{2}\left[\lim _{n \rightarrow \infty}\left(\tan ^{-1}(n)\right)\right]=\frac{1}{2}\left(90^{\circ}\right)=45^{\circ}
$$

Equation 3.3

Therefore, the critical maximum principal stress in the web shear regions should be a tensile stress at approximately $45^{\circ}$.
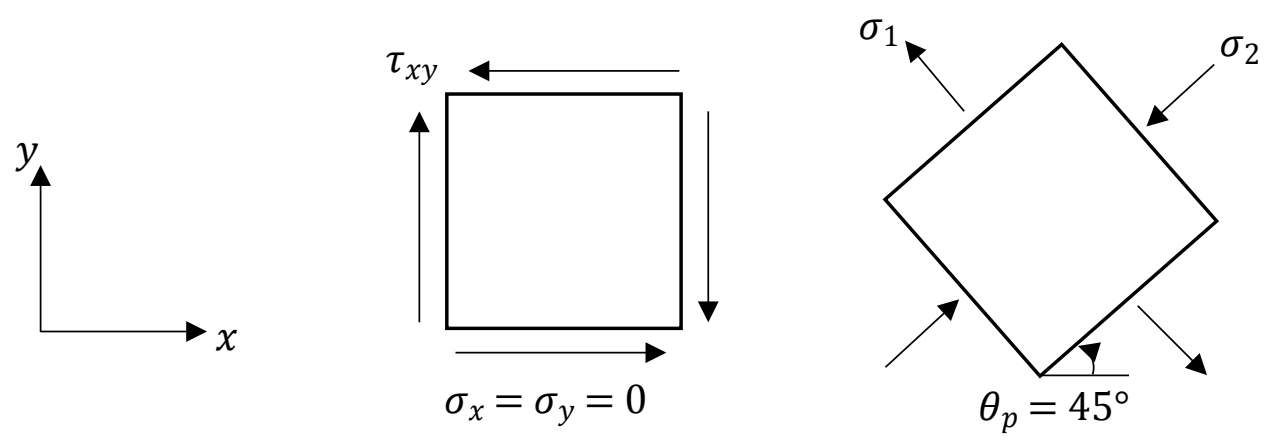

Figure 3.12: Plane stress transformations for pure shear condition

In order to determine the tensile strength before rupture at $45^{\circ}$ to the grain which runs in the longitudinal direction of the beam, Hankinson's generalized formula for tensile strength at an angle to the grain was used, as shown in Equation 3.4 (Hankinson 1921, Kollmann and Côté 1968). 


$$
f_{t, \theta}=\frac{f_{t, \|} f_{t, \perp}}{f_{t, \|} \sin ^{n} \theta+f_{t, \perp} \cos ^{n} \theta}, \quad 1.5 \leq n \leq 2
$$

Equation 3.4

With the manufacturer specified tensile strength parallel to grain $\left(f_{t, \|}\right)$ of $33 \mathrm{MPa}$ and assumed tensile strength perpendicular to grain $\left(f_{\mathrm{t}, \perp}\right)$ of $1.4 \mathrm{MPa}$ (Ardalany et al. 2011), the tensile strength at $45^{\circ}$ to grain could range from $2.26 \mathrm{MPa}$ to $2.69 \mathrm{MPa}$ (for the full range of $n$ ). Thus, the maximum principal stress for crack initiation in the shear region was defined as $2.26 \mathrm{MPa}$, with a tolerance of 0.05 . The tolerance is set so that if the stress in an element increases to over $5 \%$ of the maximum principal stress in one increment, Abaqus will cut back the time step to more accurately capture when the crack will nucleate.

The crack domain was defined as four separate enriched features in the model, as only one crack may initiate in any one enriched feature (unless two elements reach the damage initiation criteria in the same time increment). Multiple crack domain configurations were considered including the entire beam, the shear regions of the beam, and four individual symmetrical crack domains defined in the shear area of the webs at each end of the beam. These areas were defined as a "special" crack interaction using the XFEM option, with a fracture criterion interaction property defined as the contact property. The fracture criterion contact property was defined using the BK law for mixed-mode fracture energies, using parallel to the local $\mathrm{X}$-axis as the direction of crack growth (that is, the crack front is perpendicular to the primary longitudinal axis inducing crack growth along the grain). The default values of tolerance (0.2) and viscous regularization (0.00001) were used. For mode I cracking (opening normal to the crack surface) a critical energy release rate of $300 \mathrm{~N} / \mathrm{m}$ was used, based on an averaged value from experiments done on spruce (most similar 
species) by Reiterer et al. (2002). For mode II cracking (shearing along the longitudinal direction of the crack surface), a fracture energy of $730 \mathrm{~N} / \mathrm{m}$ was used based on (Kováčiková et al. 2016). Mode III cracking (shear tearing in the transverse direction of the crack surface) is rarely seen, thus a value of $10000 \mathrm{~N} / \mathrm{m}$ was used to ensure this fracture mode does not occur (Kováćiková et al. 2016). However, the Abaqus documentation states that problems in the analysis may arrive if there is a large difference between Modes II and III fracture energies so this may have caused issues in the simulation progression. An exponent of 2 was used in accordance with a brittle composite matrix (Benzeggagh and Kenane 1996) as there is little knowledge of this empirical criterion for wood. All fracture energies and the BK exponent should be further inquired in future modelling endeavors. With these parameters, a crack formed in the shear region as is shown in Figure 3.13 (deformations exaggerated by a factor of 5). The resulting mid-span deflection was 21.1 $\mathrm{mm}$, virtually the same as the purely elastic analysis.

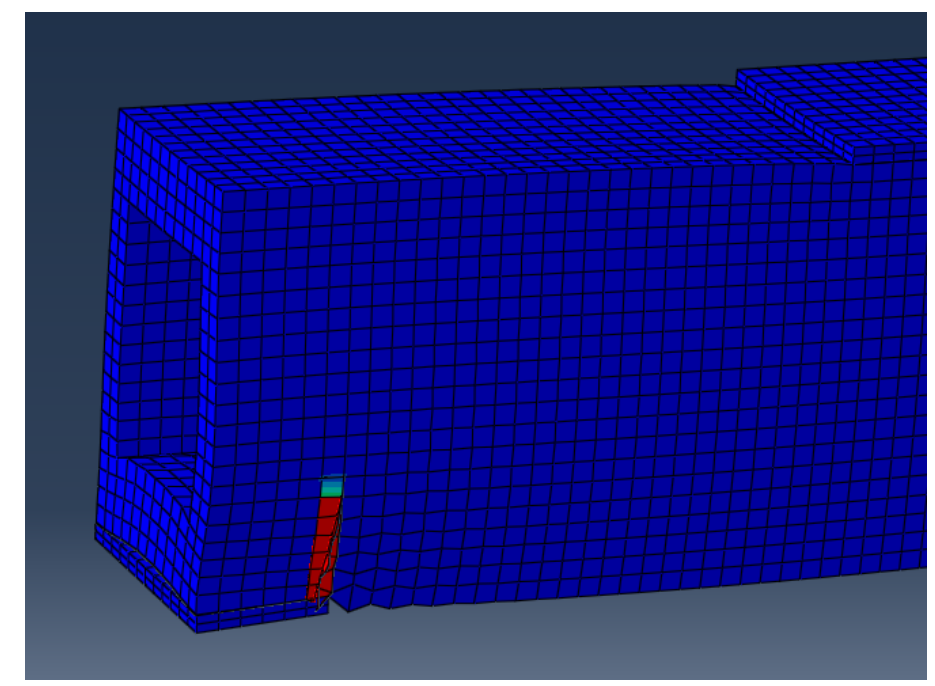

Figure 3.13: Resulting crack from XFEM analysis with deformations magnified $5 x$ Although cracks formed, the resulting behaviour did not improve from the purely linear elastic analyses using engineering constants. Additionally, many assumptions were made 
in the input parameters which would require further experimentation to validate. Therefore, it was decided that the XFEM model would not be considered further in this study.

\subsubsection{Mesh Study}

A study was done on model $\mathrm{C} 1$ with $3 \mathrm{D}$ continuum stress elements, in order to investigate the effects of varying element types and sizes on the model results. Model C1 was first investigated as a purely elastic, geometrically linear analysis with no post-tensioning, as a preliminary step. First, the cross-section mesh size was investigated. The thickness of the webs and flanges of this beam were $45 \mathrm{~mm}$ and $90 \mathrm{~mm}$, respectively, and the beam span was just over $3.5 \mathrm{~m}$ (Costello 2013). Initially, two cross-sectional mesh sizes were compared with a constant longitudinal element size of $100 \mathrm{~mm}$. The cross-section element sizes compared were $25 \mathrm{~mm}$ and $5 \mathrm{~mm}$, and the difference between the results was only $0.1 \%$. In addition to this, the computational time of the larger cross-sectional element size was less than $1 \%$ of the time required for the smaller $5 \mathrm{~mm}$ cross-section elements. Thus, a cross-section mesh size of $25 \mathrm{~mm}$ was adequate and was used for all subsequent ambient models. It was also ensured that there were at least two elements through the thickness of webs and flanges where the elements were thinner than $50 \mathrm{~mm}$.

Furthermore, several different element formulations were considered. Quadratic and linear elements were compared, using both the full and reduced-integration types. A comparison is shown in Figure 3.14. The quadratic elements and the reduced-integration linear elements showed very similar results which were slightly better than the linear elements. The linear reduced-integration elements had the lowest computational time, with results only off by $0.5 \%$ from the quadratic elements. Therefore, linear reduced-integration three- 
dimensional continuum stress elements (C3D8R) were used for all subsequent analyses in this project.

Next, a mesh refinement study of the longitudinal mesh size was completed. A variety of element sizes were compared as are shown in Figure 3.15. The study demonstrated that there was not much difference for larger longitudinal mesh sizes for purely elastic analyses. There was virtually no difference between the results for the $15 \mathrm{~mm}$ and $25 \mathrm{~mm}$ longitudinal element sizes, while the $25 \mathrm{~mm}$ size took only $60 \%$ of the computational time compared to the $15 \mathrm{~mm}$. The larger longitudinal mesh sizes also had very little difference to the $25 \mathrm{~mm}$ size, with the $100 \mathrm{~mm}$ mesh only having a difference of $0.4 \%$ less deflection.

The attention was then turned to a comparison of longitudinal mesh size with the Hill's Potential Function for plasticity added to the material model, as discussed in Section

\subsubsection{3.}

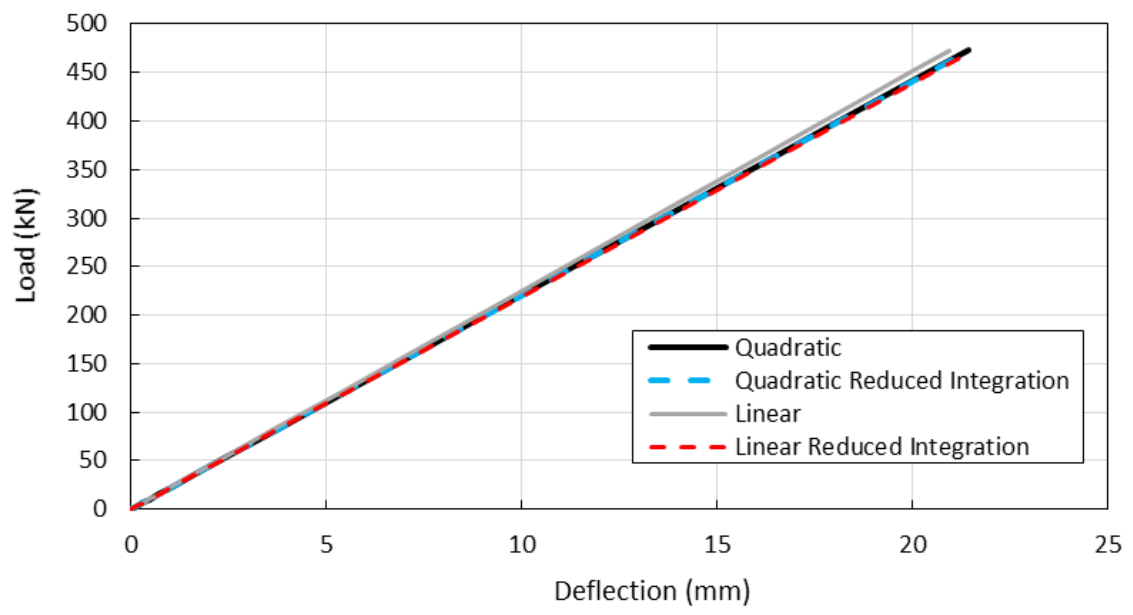

Figure 3.14: Element type comparison for model C1 with a purely elastic analysis 

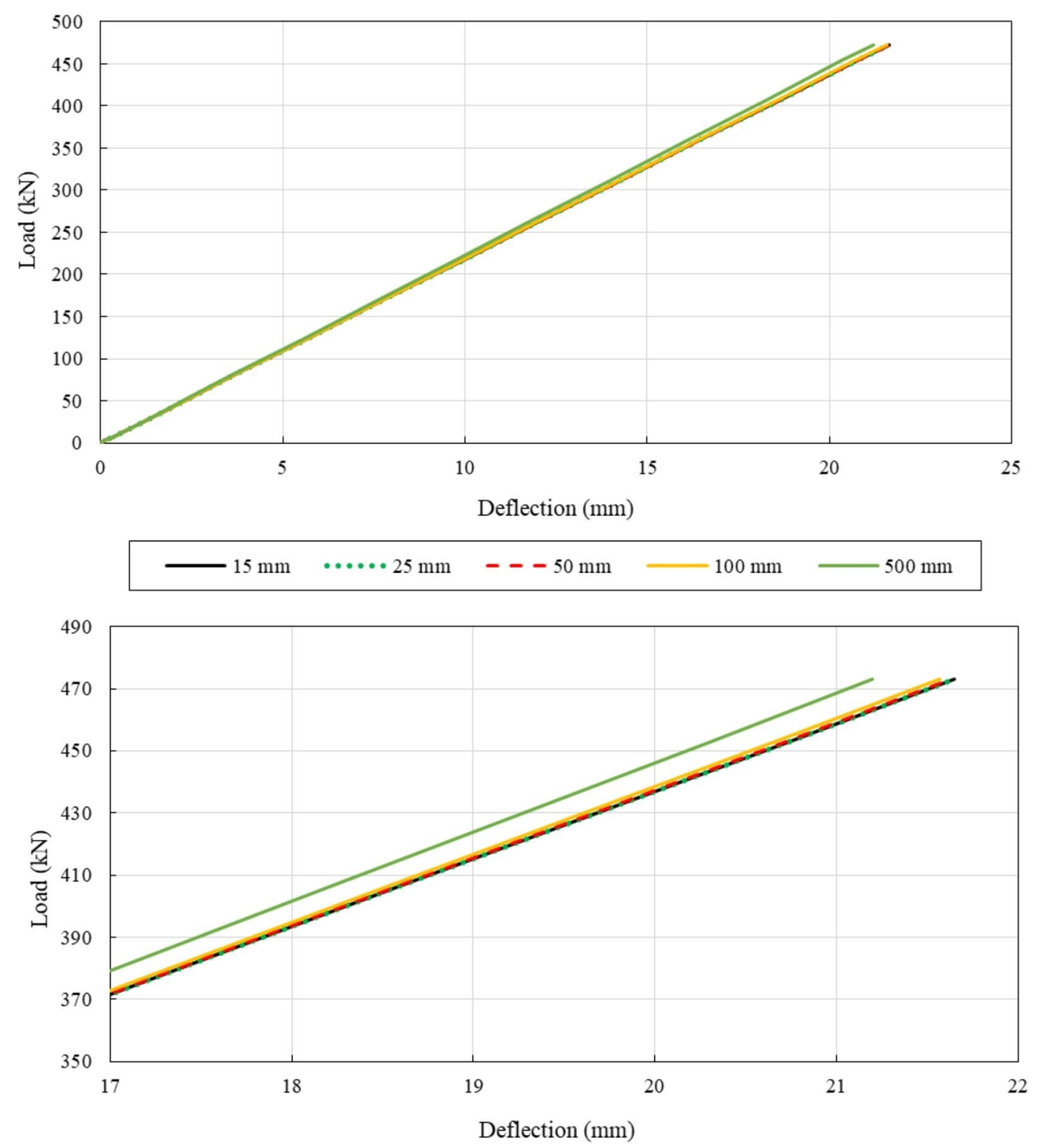

Figure 3.15: Longitudinal mesh refinement study of purely elastic analysis for model C1, with the full analysis (above) and the end of the analysis magnified to show variance (below)

The longitudinal mesh refinement study results with the Hill's Potential Function implemented are shown in Figure 3.16. 


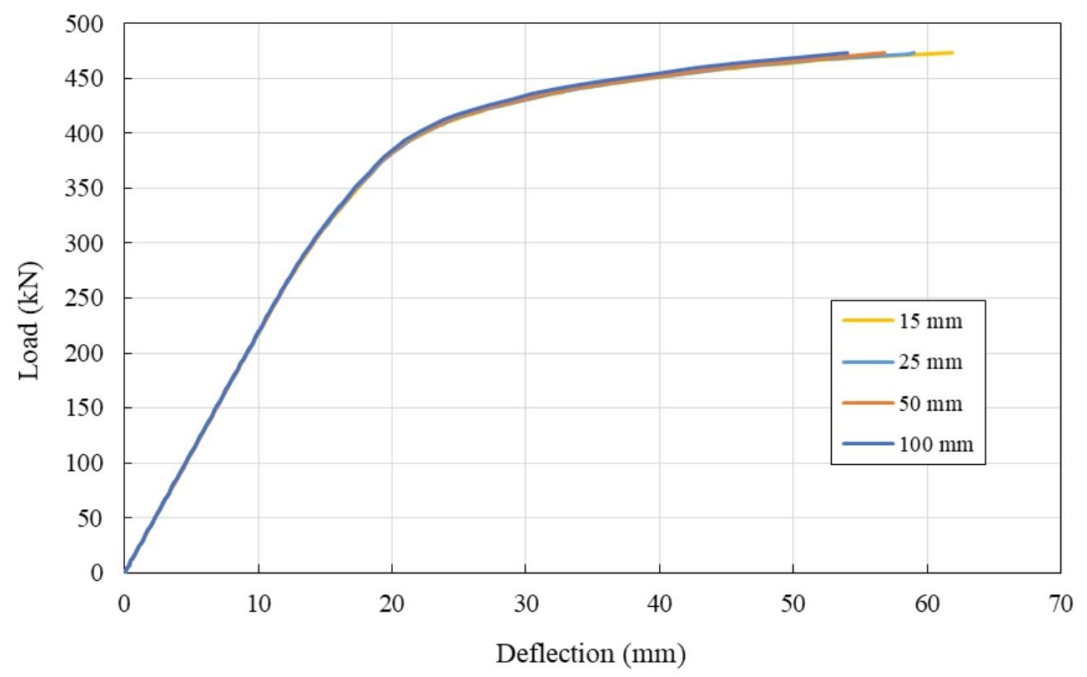

Figure 3.16: Longitudinal mesh refinement study for model C1 with Hill's Potential Function for plasticity implemented

It is shown in Figure 3.16 that the numerical results are still very much identical for all longitudinal mesh sizes considered until the end of the analysis. However, towards the end of the analysis, the smaller the element size used, the more the resulting deflection at the peak load. The load-deflection curves seem to have almost reached a plateau by the peak load, however, and the amount of runaway deflections that occur are of little consequence. The $25 \mathrm{~mm}$ mesh size required less than a quarter of the computation demand of the 15 $\mathrm{mm}$ mesh, and the final deflections were off by less than $3 \mathrm{~mm}$. Additionally, it is important for the longitudinal dimension of brick elements to not be shorter than the cross-sectional dimensions, in order to retain proper shear behavior. Due to these facts, and diminishing returns on the larger mesh sizes, it was determined that a longitudinal element size of 25 mm would give adequate results.

\subsubsection{Comparison of Results to Experiments}

The numerical results for the ambient models are compared herein to the ambient experimental data (models $\mathrm{C} 1$ to $\mathrm{C} 4$ ) in the form of load versus deflection plots. Due to the 
limited success of the CDP and XFEM material models, the results were only compared for the first model (C1).

\subsubsection{Model C1}

Comparisons between the load-deflection behaviour of multiple simulations with varying material models and the experimental results are shown in Figure 3.17. Due to the limitations discussed in Section 3.4.2.3, the results for the CDP and XFEM models were identical to their purely-elastic counterparts. Thus, one trend is shown to represent both.

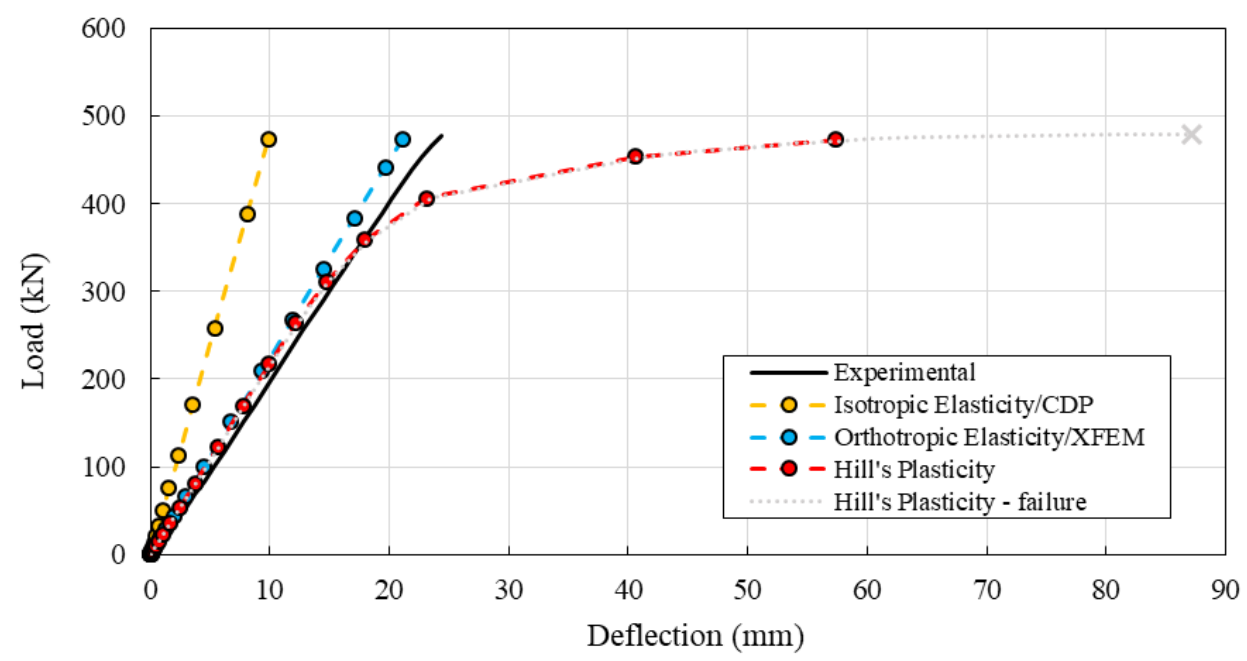

Figure 3.17: Comparison between C1 numerical model results and experimental data comparing multiple material models, where CDP gave identical results to Isotropic Elasticity and XFEM gave identical results to Orthotropic Elasticity (Costello 2013)

As may be observed in Figure 3.17, the analyses which employed the isotropic material model and implemented the CDP model both greatly underestimated the deflection of the beam. As was described in Section 3.4.2.3, the XFEM model results did not change from the purely elastic results (although some small cracks did begin to form). The orthotropic elastic model provided agreeable results with the experimental data, and the Hill's 
plasticity model followed the load-deflection behaviour until the end when the deflections greatly exceeded the experimental values.

The stresses that developed in the simulation with the Hill's Potential Function for Plasticity were inspected to ensure the assumptions made in Section 3.4.2.3 were acceptable. The shear stresses reached their failure strength in the majority of the shear region on the beam, but neither the longitudinal compressive or tensile stresses reached their yield point until the very last step of the simulation. In the prior step, the load was at $453 \mathrm{kN}$ (compared to the peak load of $473 \mathrm{kN}$ ), and the maximum tensile stress was 32 MPa while the maximum compressive stress was $35 \mathrm{MPa}$. Recall that the input maximum strength for the Hill's Potential Function model was $38 \mathrm{MPa}$, the manufacturer specified compressive strength, as all tensile stresses in the purely-elastic analysis were below failure. However, in the final step of the analysis, the tensile stresses increased to $38 \mathrm{MPa}$ due to load redistribution as the compressive and shear zones reached failure. This is an issue with the way the material model must be defined and is a shortcoming of using the Hill's Potential Function for plasticity, where tensile and compressive strengths cannot be differentiated.

The failure mode of the experiment beam was a large crack in the shear region that extended to the center of the beam. As the Hill's plasticity model is not able to predict crack initiation or propagation, this type of failure it not seen in the simulation. The failure began to manifest in the analysis as the majority of the deflections occurred in the shear region and the webs had some slight buckling near the end of the beam. Additionally, there were tensile stresses perpendicular-to-grain in the vertical direction that developed around the interior corners between the top web and flanges which were much higher than wood's 
perpendicular-to-grain tensile strength of 1.4 MPa. These are seen in Figure 3.18 to be up to almost $7 \mathrm{MPa}$ in those concentrated locations. This was contrary to what was observed in the purely elastic analyses, when tensile stresses remained well below the strength limit. The increased stresses are likely due to the deformation and outwards buckling of the webs, due to shear yielding and crushing around the supports. This indicates that if a cracking model was implemented, a crack near the web-top flange interface would likely initiate, similarly to the actual experiment failure mode.

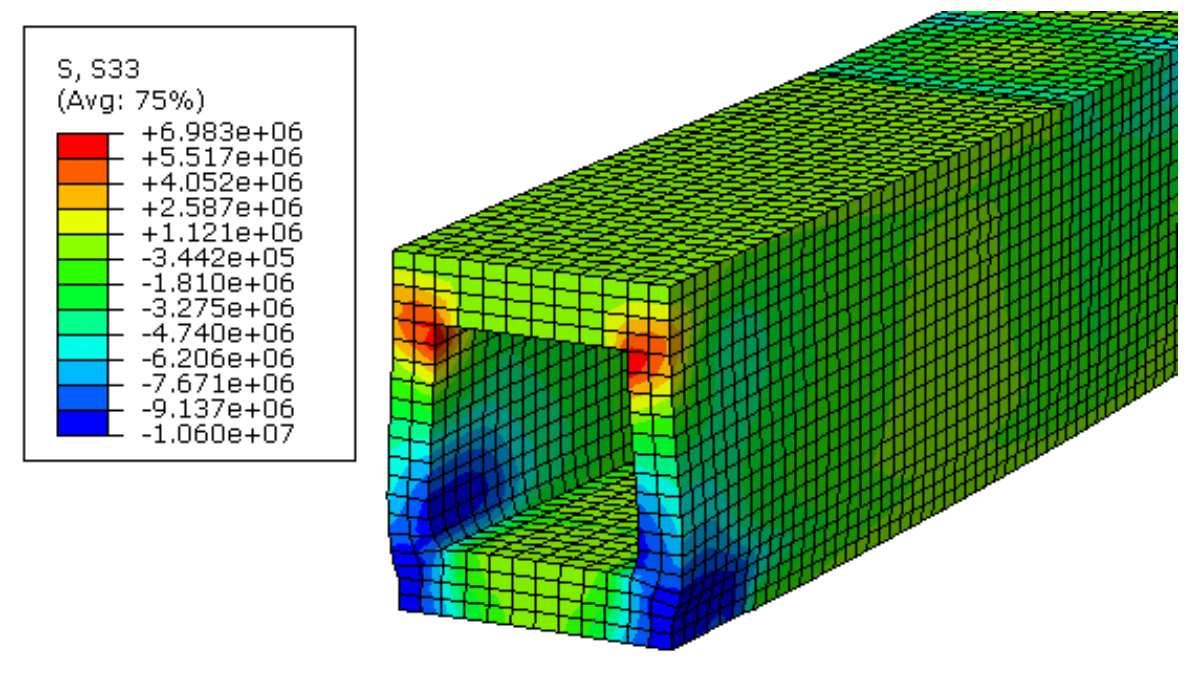

Figure 3.18: Numerical model results for $C 1$ showing stresses perpendicular-to-grain in the vertical direction, with crushing around the support and excessive tensile stresses at the top flange to web connections

Initially, the load defined into the model was input as the experimental peak load. However, if the input load on the model is simply the peak load from the experiments, the model could not be used as a predictive tool. If a larger load is input so as to fail the beam in the Hill's Plasticity model, the results are shown as the grey dashed line in Figure 3.17. The failure load predicted was $480 \mathrm{kN}$, only about $1.4 \%$ larger than the actual experimental peak load of $473 \mathrm{kN}$. The failure seen was runaway deflections which were not observed 
in the experiment as the test was terminated at the first failure point so not to damage the load apparatus and instrumentation.

\subsubsection{Model C2}

The numerical model results alongside the experimental data are presented in Figure 3.19, comparing the purely elastic model and the simulation with Hill's Potential Function for plasticity implemented. It can be seen that in both cases, the numerical model accurately estimated the force-deflection behavior for the majority of the simulation.

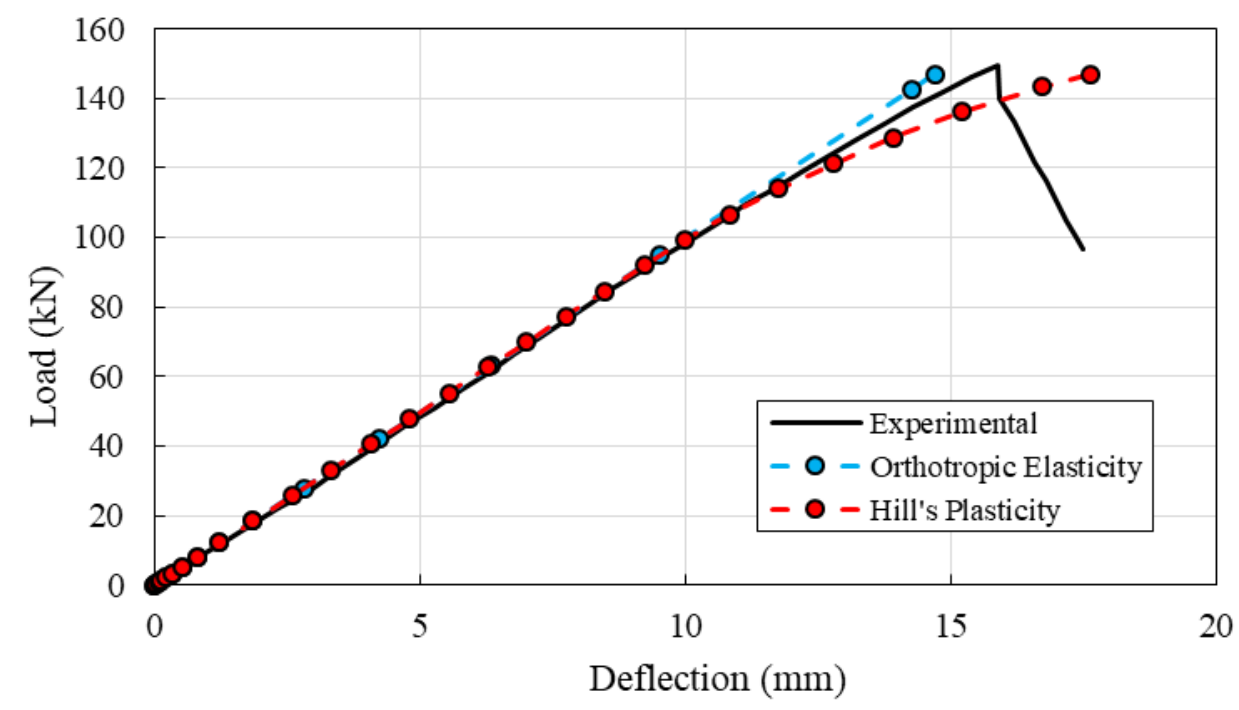

Figure 3.19: Comparison between numerical results and experimental data for C2 for models including only orthotropic elasticity and with Hill's Potential Function plasticity (Costello 2013)

The purely elastic model just falls short of the deflection at failure, while the Hill's plasticity model slightly overestimates the deflection at the peak load. However, the model does not fail at this point, the simulation ends once the input peak load is reached. In this way, the model shows decent agreement with experimental behaviour. As an attempt to observe the model failure, a load of $500 \mathrm{kN}$ was applied to the beam. Figure 3.20 shows the analysis load-deflection results. The peak load at failure in this analysis was $220 \mathrm{kN}$ 
( $50 \%$ higher than the experimental peak load of $147 \mathrm{kN}$ ) and the failure was due to excessive deformation. No decrease in applied load is seen as the simulation is load controlled which resulted in the different failure mode. Thus, the model may be used in such a way as to predict the beam behaviour below failure, but cannot be used for predictive modelling of failure modes when the peak load is unknown.

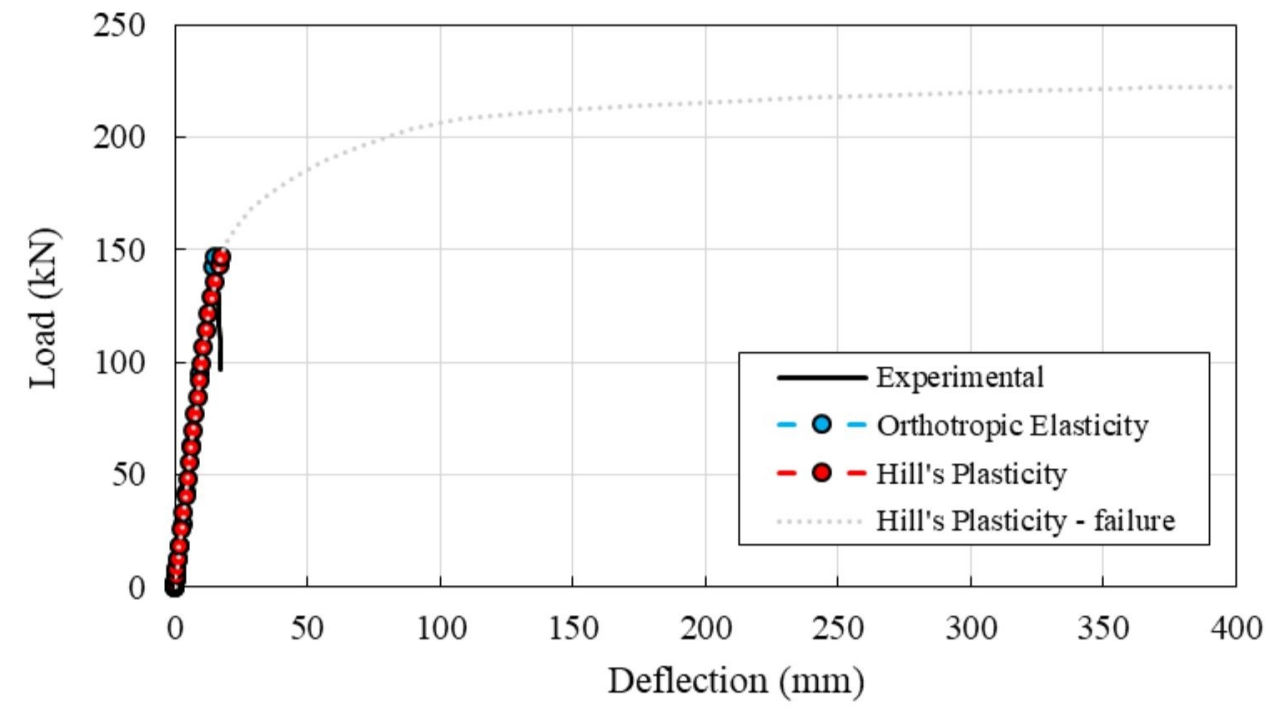

Figure 3.20: Comparison between numerical results and experimental data for $C 2$ including the attempted failure simulation (Costello 2013)

In the experiment, the failure mode was a large crack in one of the shear regions. The results showed that the material remained well within the elastic range in the compression and tension zones, but reached the yield extensively in the shear region. These stresses are shown in Figure 3.21. This failure began to manifest in the simulation by some web buckling in the shear region, and most of the vertical displacement happening in the shear region with almost constant deflection in the central bending region. This is observed in Figure 3.22. 
There was a small amount of crushing perpendicular-to-grain observed around the supports, but all the tensile perpendicular-to-grain stresses in this simulation remained below the strength magnitude.
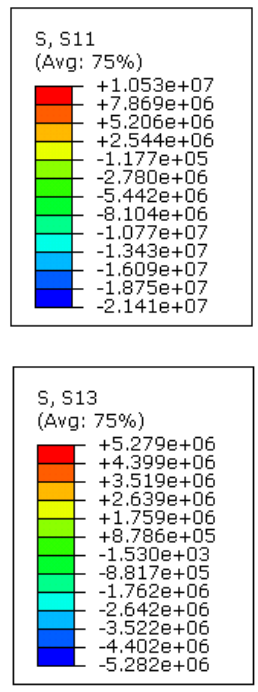

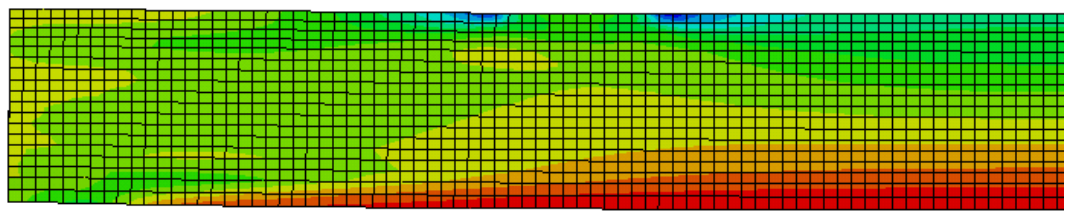

(a)

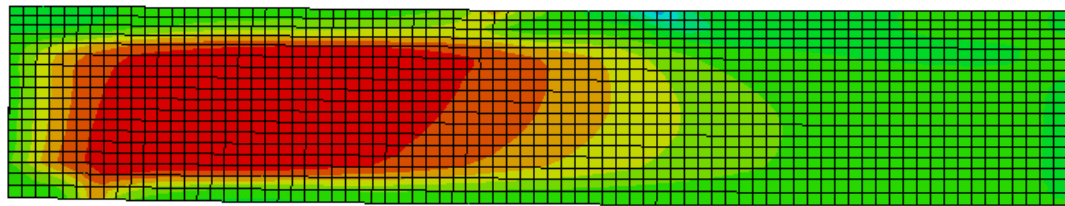

(b)

Figure 3.21: C2 Numerical model results showing half of beam shown - as stresses were symmetrical - with (a) longitudinal stresses (Pa) and (b) shear stresses ( $\mathrm{Pa}$ )
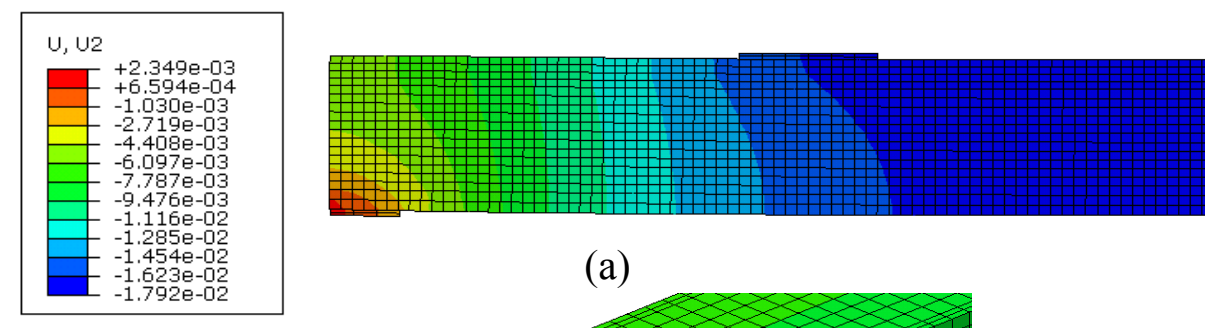

(a)
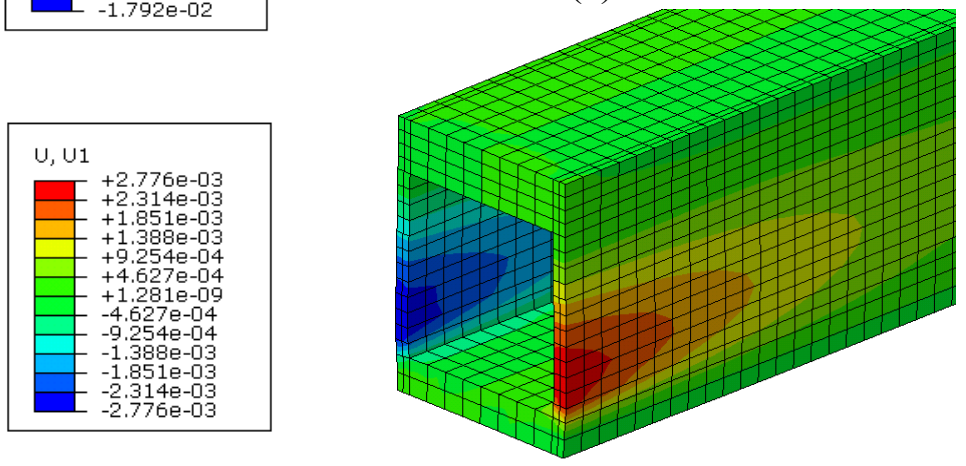

(b)

Figure 3.22: C2 Numerical model results showing (a) vertical deflection (m) - half of beam shown as deflections were majorly symmetrical, and (b) lateral deformation (m) 


\subsubsection{Model C3}

The results from the numerical model for this beam are shown compared to the experimental data in Figure 3.23. There is once again good agreement between the loaddeflection behaviour of the model and experiment in the elastic range. The model began to fail slightly earlier than the experiment, and the load does not decrease as the experiment does because the simulation is load controlled and is only programmed to increase the load until the peak. In a stroke controlled simulation, the same behaviour is observed and the load still does not decrease as observed in the experiment.

This model showed a very high crushing load around the supports due to the high peak load and the smaller span. The plasticity algorithm began to fail at increment 22 where the simulation was $90.4 \%$ complete. After this point, the software was able to converge to the end of the simulation with several iterations. However, the deformations that occurred were highly unrealistic. If the failure point is considered as the first plasticity issue in the analysis, before the runaway deflections occur, the results compared to the experimental data are shown in Figure 3.23. Thus, the model failed below the experimental peak load, at $465 \mathrm{kN}, 10 \%$ lower than $515 \mathrm{kN}$. This is in contrast to the other ambient models which all failed at loads above the experimental peak load. 


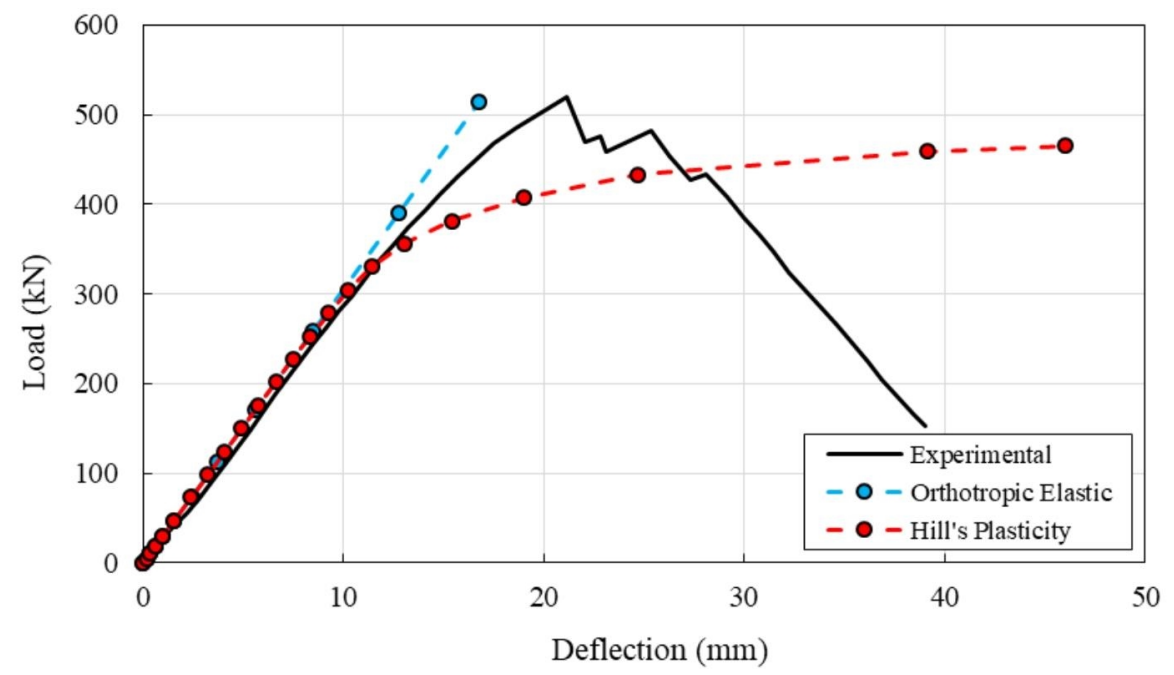

Figure 3.23: Comparison between numerical results and experimental data for $\mathrm{C} 3$ for models including only orthotropic elasticity and with Hill's Potential Function plasticity implemented (Costello 2013)

The longitudinal tensile and compressive stresses in this analysis remained below the failure strength and within the elastic range. There was ample yielding in the shear region, and a large amount of crushing very early on in the simulation around the supports. Past the assumed failure point discussed above, there was unrealistic deformations around the supports due to this condition. At least until the failure point, the tensile perpendicular-tograin stresses all remained below the strength of 1.4 MPa.

\subsubsection{Model C4}

A comparison between the results for load-deflection behaviour to the experimental data is presented in Figure 3.24. This model included the post-tensioning, implemented as described in Section 3.4.1. However, the results are shown with the deflections zeroed after the post-tensioning to match the presentation of the experimental data available. It is observed that here, unlike the other ambient simulations, the flexibility of the beam was 
slightly underestimated. However, the deflection was overestimated at the experiment peak load by almost $6 \mathrm{~mm}$.

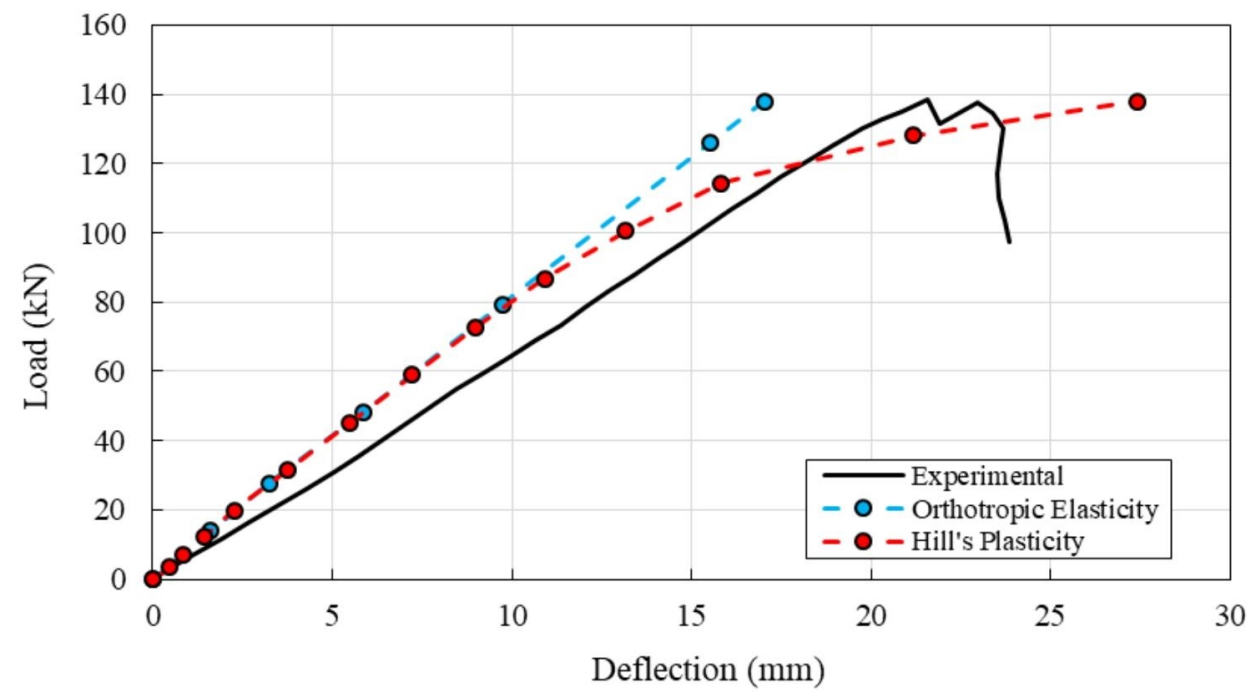

Figure 3.24: Comparison between numerical results and experimental data for C4 for models including only orthotropic elasticity and with Hill's Potential Function plasticity implemented; deflections normalized after post-tensioning (Costello 2013)

In this model, the compressive stresses in both longitudinal and perpendicular-to-grain directions reached their respective yield strengths, showing some crushing around the supports and load plates. Additionally, both the longitudinal and perpendicular-to-grain tensile stresses did not reach their respective strength magnitudes. This shows that for the model with pre-stressing, the Hill's plasticity model input assumptions were acceptable as the tensile stresses remained in the elastic range.

A failure analysis was also done in order to observe the ability of the model to predict failure behaviour of the beam. The plasticity algorithm began to fail at increment 25 where the simulation was $5.1 \%$ above the experimental failure load. The failure mode was a shear failure in both shear regions. After this point, the software was able to converge up to $20 \%$ over the experimental peak load with several iterations, however, the deformations that 
occurred were highly unrealistic, reaching over $2 \mathrm{~m}$ which would not be possible in reality. If the failure point is considered as the first plasticity issue in the analysis, before the runaway deflections occur, the results compared to the experimental data are shown in Figure 3.25. Once again, the deflections were overestimated, however the results show decent agreement with the experimental data, with a failure load of $145 \mathrm{kN}$ compared to the experimental peak load of $138 \mathrm{kN}$.

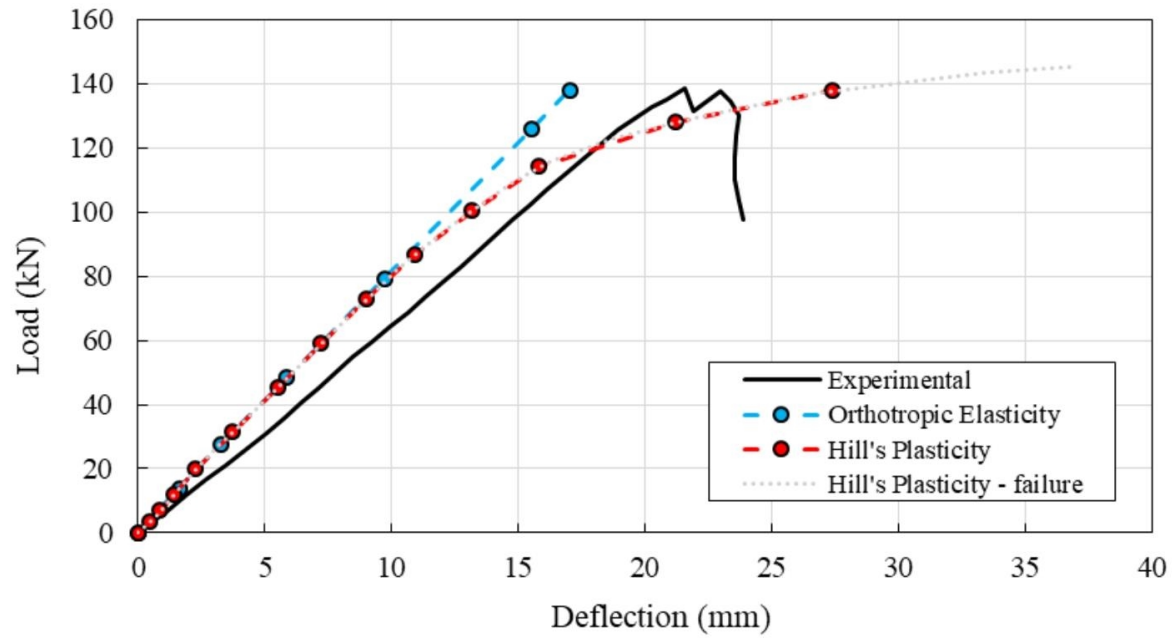

Figure 3.25: Comparison between numerical results and experimental data for C4 including the attempted failure simulation; deflections normalized after post-tensioning (Costello 2013)

\subsection{Heat Transfer Modelling}

The thermal analysis is the first step in a structural fire simulation where the heating regime is applied to the exposed surfaces of the structural element and a transient heat transfer analysis is completed. From here, the time-history results of nodal temperatures may be used as input predefined fields for structural analyses. 


\subsubsection{Model Setup}

Only the timber beam was modelled in the thermal analysis with three-dimensional, linear heat transfer elements DC3D8. Additionally, the cross-section was modelled as twodimensional, linear heat transfer elements DS4 for a separate mesh study as the crosssectional mesh is the most important and a two-dimensional study greatly decreased computation time. The linear elements were used due to the discontinuity in the analysis from the latent heat of evaporation of water. Model attributes were added for absolute zero temperature as $-273.15^{\circ} \mathrm{C}$ to ensure the analysis runs in Celsius, and the Stefan-Boltzmann constant as $5.67 \times 10^{-8} \mathrm{~W} / \mathrm{m}^{2} \mathrm{~K}^{4}$ for radiative heat transfer.

In the initial step, a predefined field was defined for the initial ambient temperature in each analysis. A second heat transfer step was added to apply the fire exposure to the beam. The heat exposure was applied only to the three exposed surfaces of the beam as the top was protected with the furnace roof and loading apparatus. Additionally, the ends of the beams which were over the supports and outside the furnace were left unexposed. The unexposed surfaces were unmodified and thus assumed to be adiabatic. An example of the exterior surface heat exposure on model C5 is shown in Figure 3.26. 

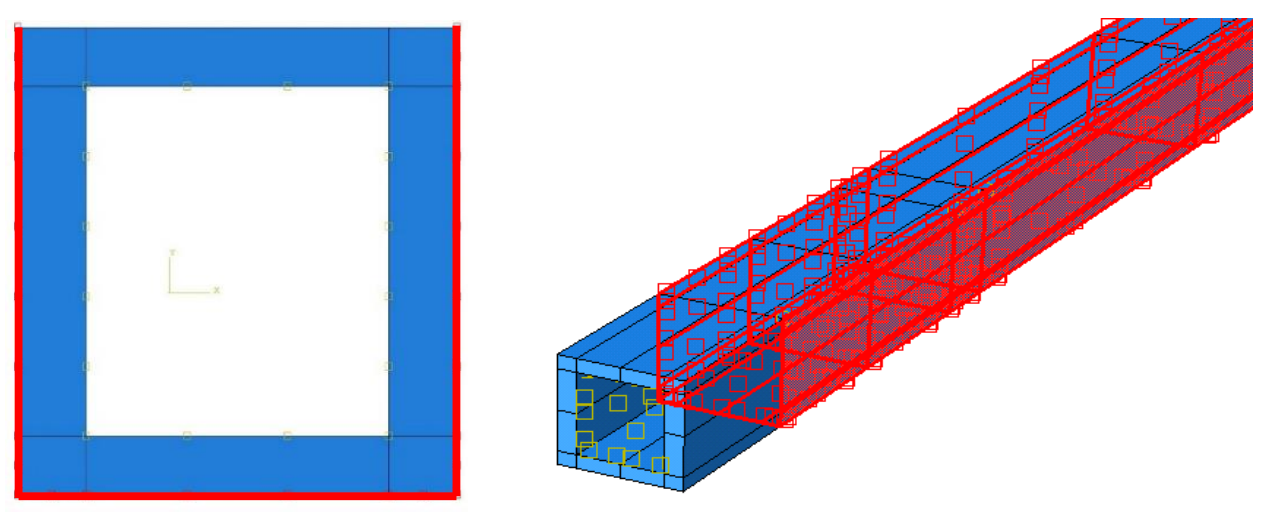

Figure 3.26: Heat exposure applied on surfaces shown in red on $2 D$ and $3 D$ analyses, where the end of the beam outside the furnace and the top surface was left unexposed The fire curve applied was the standard fire curve (ISO 2014) as described by Equation 3.5 , the time duration varying depending on the experiment.

$$
T(t)=T_{a}+345 \log (8 t+1)
$$

Equation 3.5

A tabular set of data for each simulation was set up using Equation 3.5 with time steps of 30 seconds for the entire experiment duration and was defined in Abaqus as an Amplitude. The fire was applied to the surfaces shown in Figure 3.26 by convection and radiation interactions. For the convection interaction, a "Surface film condition" was defined with an Embedded Coefficient of $25 \mathrm{~W} / \mathrm{m}^{2} \mathrm{~K}$ which is common for a standard fire furnace test, with an instantaneous amplitude. The Sink Temperature was defined as uniform with a magnitude of one multiplied by the Amplitude curve previously defined as the standard fire. For the radiation interaction, a "Surface radiation" was defined to ambient with a uniform emissivity of 0.8 for wood. Similarly to the convective interaction, the Ambient Temperature was defined as one multiplied by the standard fire curve Amplitude. 
In addition to the surface convection and radiation to the furnace time-temperature curve, the interior cavity surfaces were defined with radiative properties in order to simulate heat transfer between the surfaces in the cavity. Abaqus offers a cavity radiation interaction in which the cavity surfaces are defined and the view factors between each element on the cavity surface are calculated, thus allowing the radiative heat transfer between each discrete element in the cavity to every other element. This was used in the two dimensional mesh study in order to capture heat transfer between the cavity edge elements. Due to the large size of the three-dimensional beam model, a cavity approximation was used rather than true cavity radiation, due to the significant computational demand of calculating view factors. The cavity approximation is available within the regular "Surface radiation" interaction and is chosen rather than the "To ambient" selection. Rather than calculating view factors, the cavity approximation calculates the average surface temperature within the cavity at each step and uses this to define the "ambient temperature" for the radiative heat transfer. This temperature is then used to determine the heat transfer to or from each element on the cavity surface. The approximation was used because the cavity surfaces are relatively isothermal throughout the simulation and the emissivity of the surface is uniform throughout the cavity. The results were compared to the true cavity radiation results from the $2 \mathrm{D}$ analysis and any differences were negligible. The surfaces to which the cavity radiation was applied are shown in Figure 3.27. 

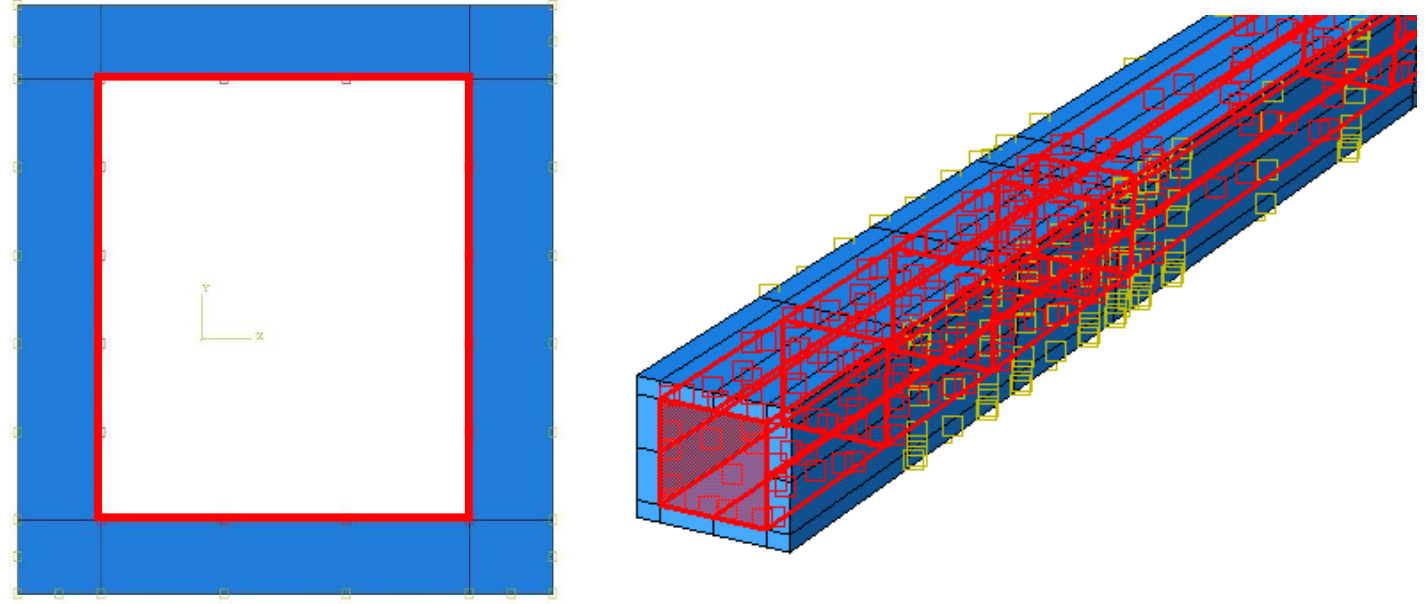

Figure 3.27: Radiation applied on cavity surfaces shown in red on $2 D$ and $3 D$ analyses

\subsubsection{Material Model}

Wood was the only material considered in the heat transfer modelling as no steel elements were exposed to extreme heating. The steel tendons remained well below $200^{\circ} \mathrm{C}$ which is the lowest temperature where their strength would be effected by heat. The highest interior cavity temperatures reached at the end of all furnace tests was around $100^{\circ} \mathrm{C}$, at which the strain from thermal expansion would be less than $0.1 \%$. This stress may be significant in relaxing the tendon force and affect the model results. However, the addition of this would introduce many complexities such as dependant heat sinks and localized heating due to cracks and heat infiltration through the timber which were not considered in this model. Additionally, a simplified calculation method was developed by Costello (2013) in which it was determined that the tendon temperature had negligible effects on the beam performance and failure. Therefore, the thermal expansion of the steel tendons was outside the scope of this model and the effects of this on the tendon force results are discussed in Section 3.6.3. 
The changes in wood material as it is heated to volatile combustion gases and char, and eventually to a complete loss of material around the exposed surfaces, make the material very difficult to model in relatively simple heat transfer analyses. In many cases, effective thermal properties such as those suggested in Eurocode 5, Part 1-2, Annex B, are used in order to capture all those phenomena (CEN 2004b). The temperature dependent properties will change throughout the simulation at any point within the solid to represent the changes in thermal conduction, heat capacity and density due to numerous factors such as mass loss, char development and moisture migration.

In addition to these Eurocode values which use a jump in the effective specific heat curve to represent the evaporation of moisture (CEN 2004b), a latent heat method was also used to investigate differences. The moisture content (MC) of the wood specimens tested by Costello (2013) and Spellman (2012) were not specified, thus a 6\% MC was assumed, based on MC values for past wood experiments. The heat of evaporation of water is 2260 $\mathrm{kJ} / \mathrm{kg}$. Abaqus assumes that the entire sections defined by the material properties go through the phase change and absorb energy, thus it is required to convert this latent heat per mass of water to an amount of energy per mass of wet timber. This was determined through Equation 3.6:

$$
\begin{aligned}
& \Delta H_{\text {vap,wood }}=\Delta H_{\text {vap,water }} \cdot \% M C \\
& \quad=2260 \mathrm{~kJ} / \mathrm{kg}_{\text {water }} \cdot 0.06=135.6 \mathrm{~kJ} / \mathrm{kg}_{\text {wood }}
\end{aligned}
$$

Equation 3.6

A latent heat of $135.6 \mathrm{~kJ} / \mathrm{kg}$ of wood was used to represent the amount of energy required to evaporate the water within the wood at $6 \% \mathrm{MC}$. This model resulted in almost identical 
temperature profiles as the specific heat method, but with a much more stable analysis and less computational time. Therefore, the latent heat method was used for all subsequent analyses.

\subsubsection{Mesh Study}

A mesh sensitivity study was done on a cross-section for model C5 in order to determine the refinement required. Mesh sizes of $9 \mathrm{~mm}, 5 \mathrm{~mm}, 3 \mathrm{~mm}$ and $1 \mathrm{~mm}$ were tested, along with a mesh of combined element sizes shown in Figure 3.28. Figure 3.29 shows the convergence results for the mesh sizes in various locations on the cross-section, including the inside and outside surfaces mid-height of the web and the inside and outside nodes of the bottom corner.

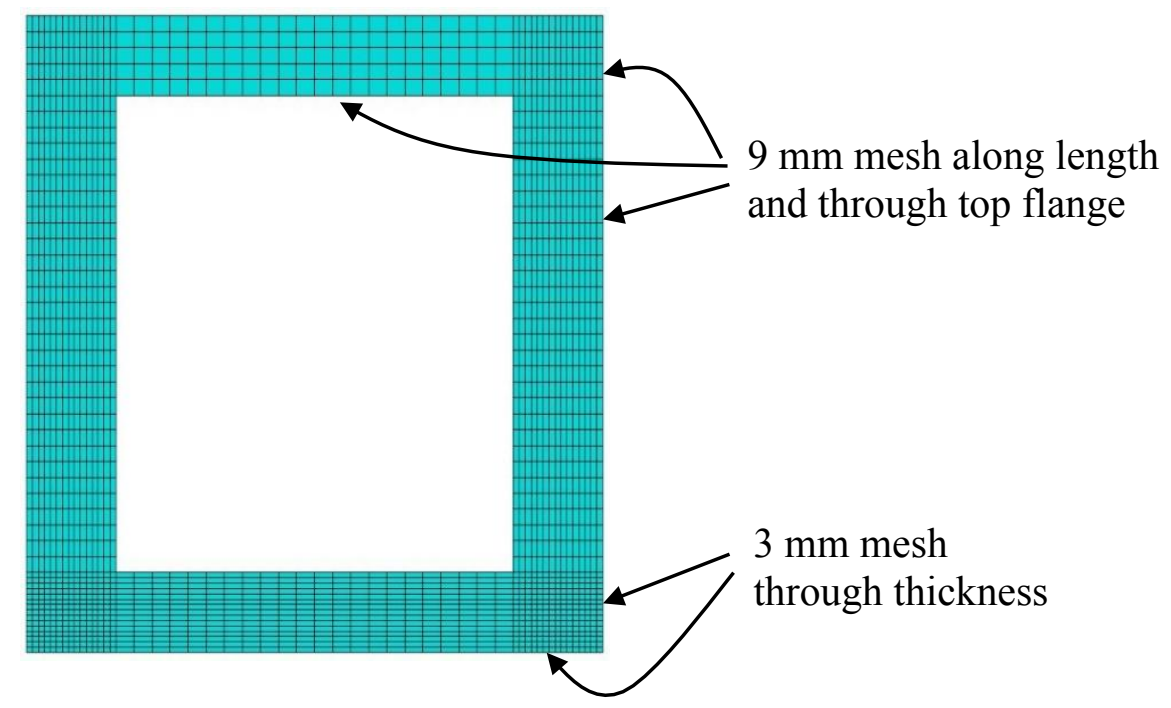

Figure 3.28: Combined element size mesh with smaller element dimensions through the thickness of the exposed flange and webs to capture more detailed heat transfer gradients 

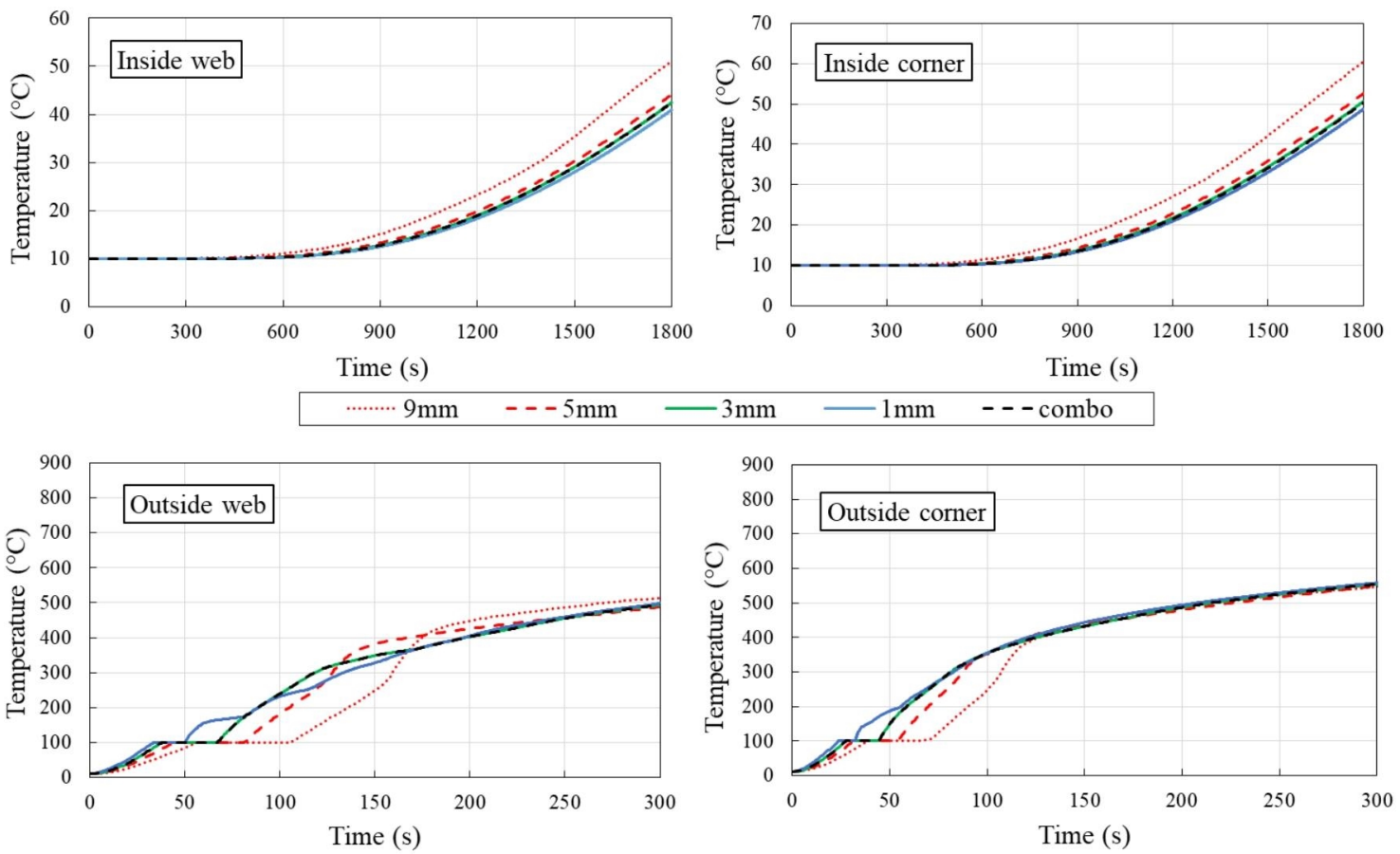

Figure 3.29: Mesh refinement study for beam cross-section thermal analysis (outside node results are shown magnified to the first 300 seconds of the analysis as mesh sizes mostly converged after this time)

As can be seen from Figure 3.29, the combination mesh results are identical to the $3 \mathrm{~mm}$ mesh results. The $1 \mathrm{~mm}$ mesh gave slightly different results for the inside surface temperatures, with a difference of about $1.5^{\circ} \mathrm{C}$ in the final temperature compared to the 3 $\mathrm{mm}$ mesh. There were also some pronounced differences between the temperatures in the outside web and corner nodes within the first 150 seconds of the simulation, and by the 300 second point all mesh sizes had converged to similar results (thus only the first 300 seconds of the simulation are shown in the outside surface plots). Although the $3 \mathrm{~mm}$ mesh had variances in the results from the $1 \mathrm{~mm}$ mesh, the simulation took only $3 \%$ of the time. Thus, the incredibly increased simulation time was not justified with the small result discrepancies. The combination mesh had identical results to the $3 \mathrm{~mm}$ mesh, but used only $30 \%$ of the computation time. Additionally, the three-dimensional heat transfer analyses 
take exponentially more computational time with smaller elements. There is little difference between the $3 \mathrm{~mm}$ and $5 \mathrm{~mm}$ results, with less than $4 \%$ error for the internal surface temperatures, and only small discrepancies of about 10 seconds in the first few minutes of the simulation for the external surface temperatures (as can be seen in Figure 3.29). Thus, the combination mesh with $5 \mathrm{~mm}$ elements through the thickness was used for all subsequent 3D thermal analyses. The longitudinal mesh size was $25 \mathrm{~mm}$ near the ends of the beam where some small amount of longitudinal heat transfer may occur towards the unexposed ends, and through the rest of the beam $100 \mathrm{~mm}$ elements were used as no longitudinal heat transfer is expected.

\subsubsection{Comparison of Results to Experiments}

A heat transfer analysis was done for each of the furnace test experiments modelled, C5, S6 and S7. These results will be compared to the available experimental data herein.

\subsubsection{Model C5}

Costello's furnace test provided limited thermal data to compare to, as the only thermocouples located on the beam were just on the inside surface of the web and on the steel tendons. In the numerical model, it was assumed that the steel tendon would be at the temperature of the inside surface of the beam cavity, which always remained below $50^{\circ} \mathrm{C}$, and thus the steel tendons were assumed to be unaffected, aside from negligible thermal expansion. Figure 3.30 shows the comparison between the available experimental results for the temperature on the inside surface of the web and the numerical model results. 


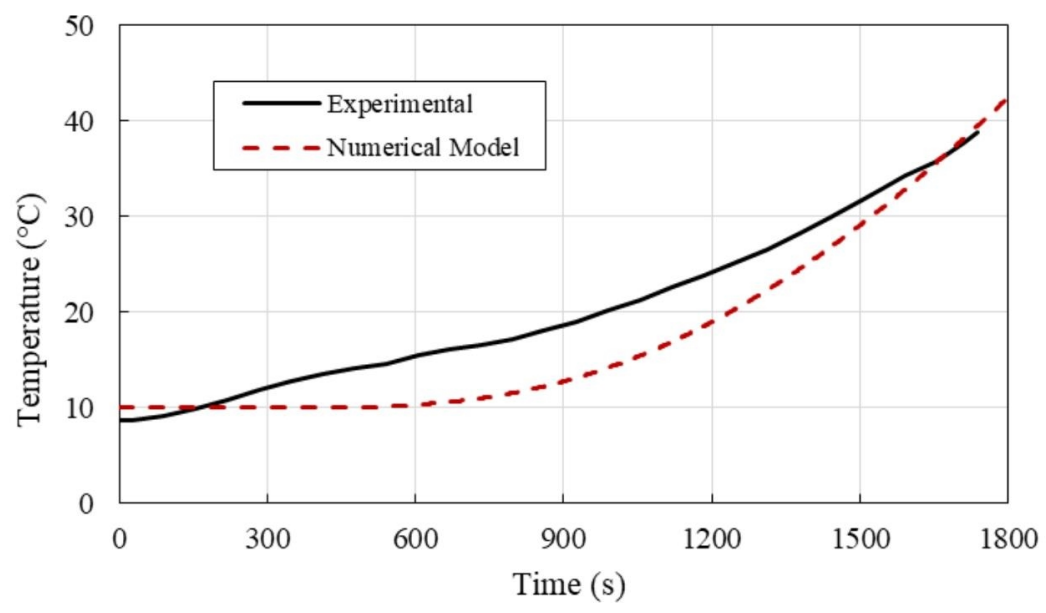

Figure 3.30: Numerical model heat transfer results for interior web surface temperature versus experimental data for C5 (Costello 2013)

The temperatures at the beginning and end of the simulation are quite agreeable with experimental results, however, the model temperatures throughout the analysis are consistently lower than the experiment by about $5^{\circ} \mathrm{C}$. This could be due to the fact that some hot smoke had infiltrated into the cavity in the experiment which would have increased the temperature reading on the thermocouple. However, the discrepancies all lie below $40^{\circ} \mathrm{C}$ and thus there would be little difference in the wood material properties.

Costello also provided an image showing the extent of charring on a cross-section of the beam, and reported $25 \mathrm{~mm}$ of char depth measured. After 30 minutes of heat exposure in the numerical model, $20 \mathrm{~mm}$ of char were measured based on the $300^{\circ} \mathrm{C}$ isotherm (as recommended in Section 3.4.1 in CEN 2004a). This is $5 \mathrm{~mm}$ less char depth than the experimental char measured, which is quite substantial. However, in reality, the beam in the furnace would have continued to char past the recorded failure time of 29 minutes, while the furnace was opened, the beam was removed, extinguished, and took time to cool 
down. This could explain the discrepancy between char depths. A comparison between the char depths at the end of the heat exposure is shown in Figure 3.31.
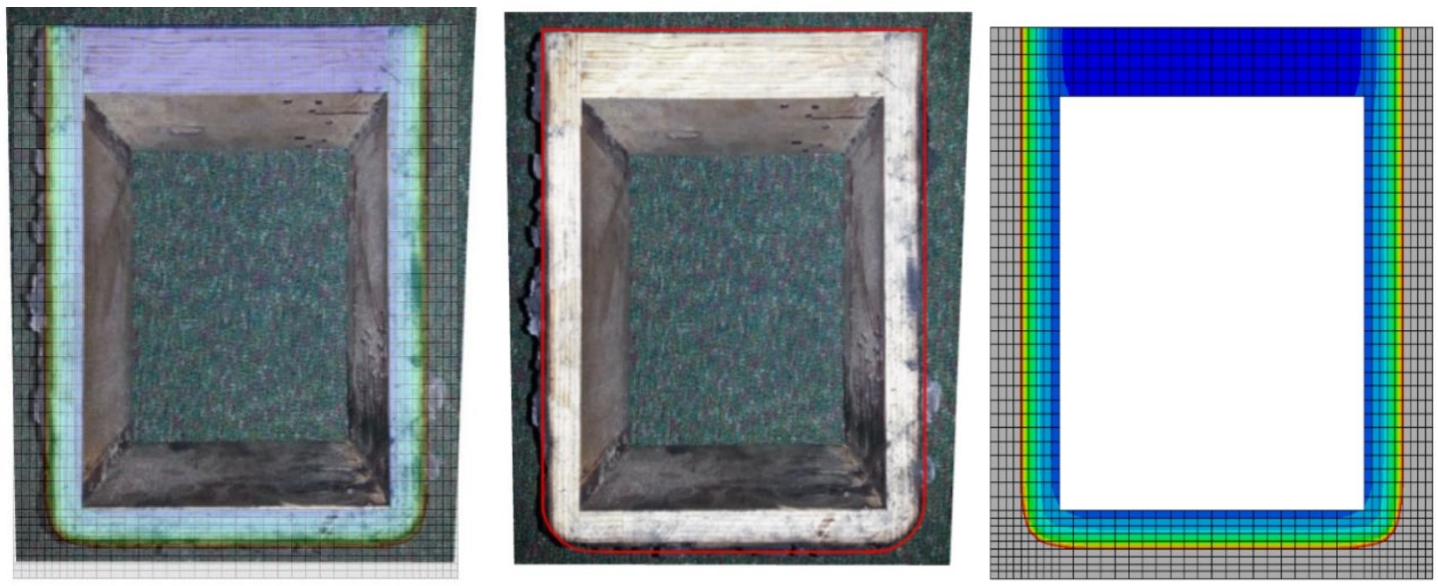

Figure 3.31: Comparison of $C 5$ char depth image - modified to fix camera angle distortion from (Costello 2013) - and numerical model heat transfer at $300^{\circ} \mathrm{C}$ maximum, with an overlay (left) and a trace (middle) of the numerical results (right)

\subsubsection{Model S6}

Spellman's furnace tests provided substantially more recorded thermal data for comparison with the thermal numerical model results. The thermocouple placement through the thickness of the webs and bottom flanges of the furnace tests were the same, with thermocouples at $0 \mathrm{~mm}, 6 \mathrm{~mm}, 17 \mathrm{~mm}, 28 \mathrm{~mm}, 39 \mathrm{~mm}, 50 \mathrm{~mm}$ and $63 \mathrm{~mm}$ (the inside surface of the box element). Due to the slightly irregular placement of the thermocouples, the cross-section of the beam was partitioned in the model to ensure nodes were placed at the proper locations. The partitioning is shown in Figure 3.32. This also resulted in varied mesh sizes through the thickness of those elements, with some elements slightly larger and some slightly smaller than $5 \mathrm{~mm}$. The longitudinal mesh was defined the same way as described above. 


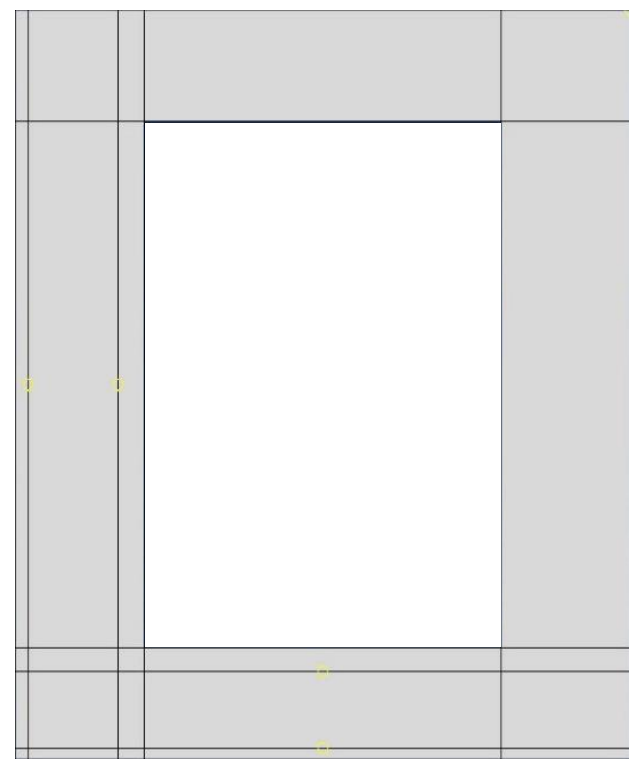

Figure 3.32: Partitions for irregular thermocouple placement where the outermost partition is $6 \mathrm{~mm}$ away from the outer surface (with one $6 \mathrm{~mm}$ element), the innermost partition is $13 \mathrm{~mm}$ in from the interior surface (with three $4.33 \mathrm{~mm}$ elements), and the middle partition having $5.5 \mathrm{~mm}$ elements to ensure a node is located every $11 \mathrm{~mm}$, corresponding to thermocouple placement

The nodal temperatures were then extracted from the model results at the center of one web and the bottom flange, at the beam mid-span, for comparison with the experimental temperatures. A comparison of the web temperatures is shown in Figure 3.33 and the bottom flange temperatures in Figure 3.34. 


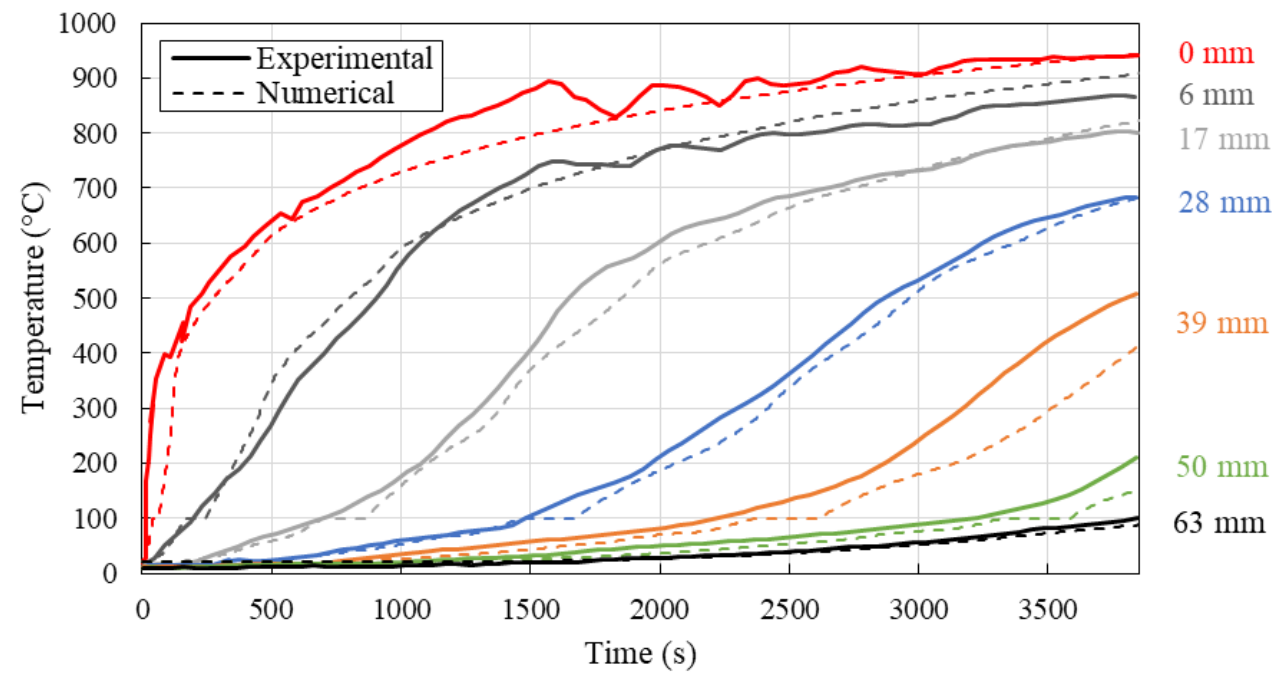

Figure 3.33: Web temperature distribution comparison for S6 experimental results versus numerical model (Spellman 2012)

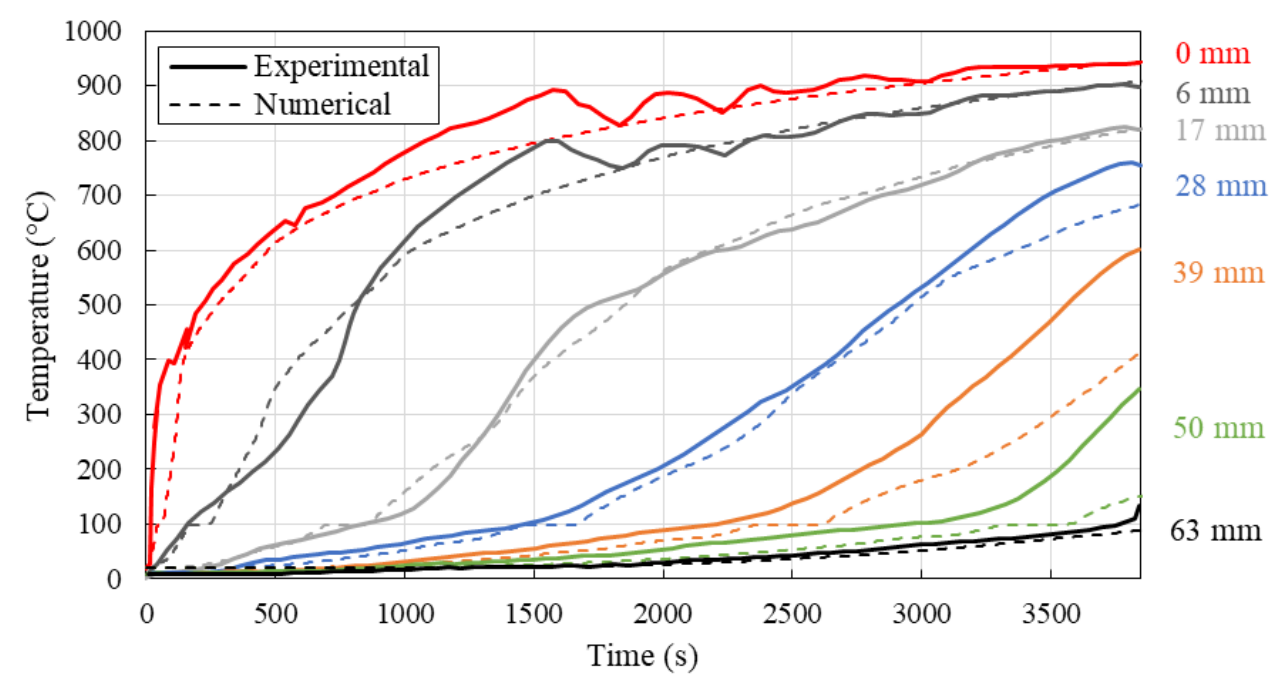

Figure 3.34: Bottom flange temperature distribution comparison for S6 experimental results versus numerical model (Spellman 2012)

As can be seen in Figure 3.33 and Figure 3.34, the numerical results compare quite well with the experiment. There is some discrepancy between the two results around the midpoint of the box elements, at the $39 \mathrm{~mm}$ and $50 \mathrm{~mm}$ thermocouples. The hotter temperatures recorded there in the experiment could be due to increased heat transfer from moisture migration which is not accounted for in the model. 
Spellman did not provide an image showing char depth in his thesis, but the reported char depth after 66 minutes of heating was $47.5 \mathrm{~mm}$. The model predicted a depth of $44 \mathrm{~mm}$ at that time, only $3.5 \mathrm{~mm}$ less than measured. It is once again difficult to ascertain the direct error in these results as the amount of charring that occurred in the experimental beam after the furnace was opened was unknown.

\subsubsection{Model S7}

The temperature recordings for Spellman's third furnace test were done similarly to the previously discussed experiment, and the model partitioning was set up in the same fashion. A comparison of the web temperatures is shown in Figure 3.35 and the bottom flange temperatures in Figure 3.36.

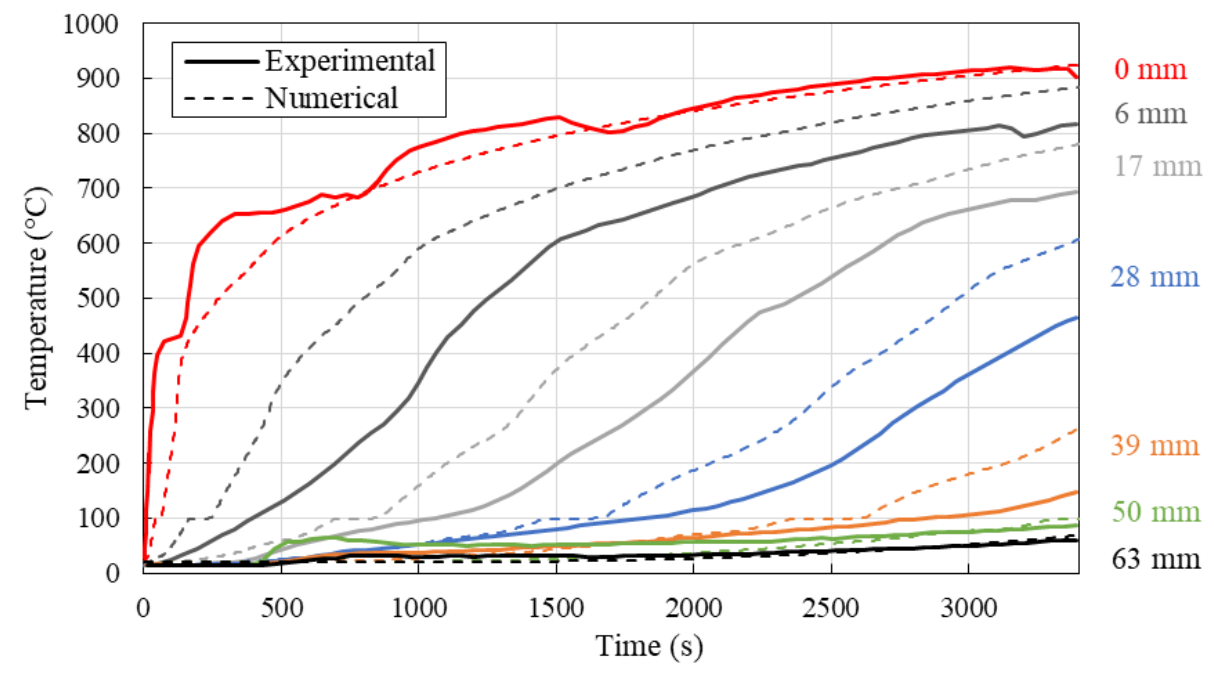

Figure 3.35: Web temperature distribution comparison for $S 7$ experimental results versus numerical model (Spellman 2013) 


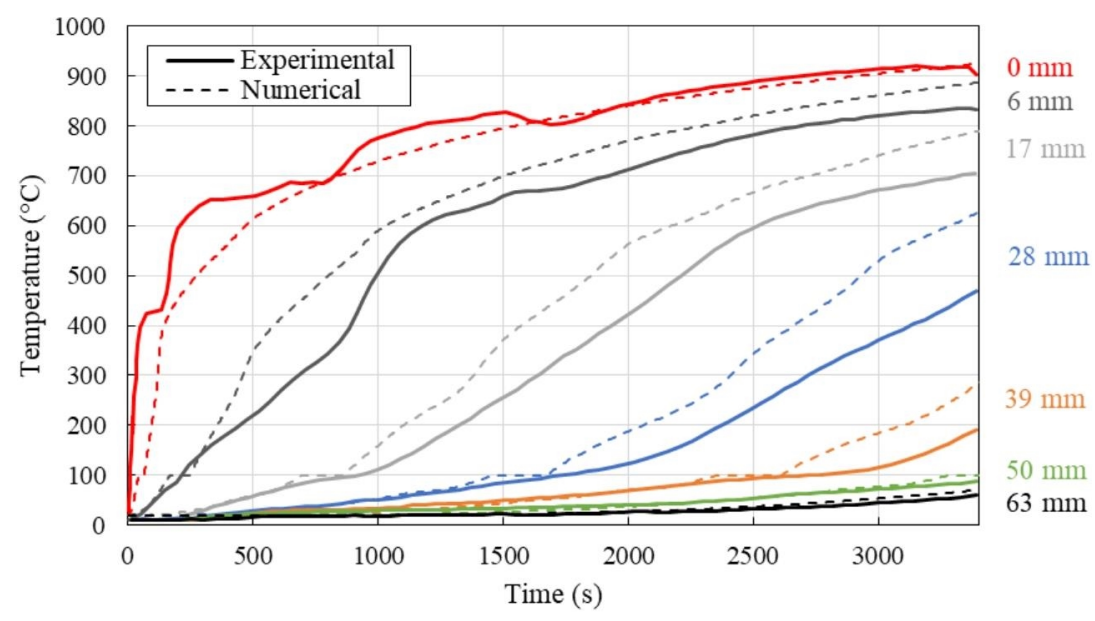

Figure 3.36: Bottom flange temperature distribution comparison for $S 7$ experimental results versus numerical model (Spellman 2013)

As can be seen in Figure 3.35 and Figure 3.36, the temperature results from the model were much higher than recorded in the experiment. However, Spellman stated in his thesis that the temperatures recorded in this experiment were abnormally low. The reported char depth for this experiment after 58 minutes of heating was $40 \mathrm{~mm}$, while the numerical model predicted a char depth of $39 \mathrm{~mm}$ at this time, showing very good agreement. These details indicates that an anomaly with the experimental data may have occurred, resulting in the large discrepancies between model and experiment temperature readings.

\subsection{Sequentially Coupled Thermal-Mechanical Analysis}

After the heat transfer analyses has been completed, the output database was used as a predefined field input for the structural analysis in which material properties are functions of temperature. By using the output database file (rather than a results file), Abaqus allows for dissimilar meshes between the thermal and mechanical analyses. Given that the part names are the same between the two models, Abaqus will use the part geometry to interpolate element temperatures in the mechanical model based on the nodal temperature 
results from the heat transfer model. The model was setup the same as described in Section 3.4.1, and the thermal results were applied to the beam in a fourth step (after the vertical loading had been applied) with all previous loads remaining constant. The temperatures at each node were applied using a Predefined Field "From Results or Output Database File", with incompatible mesh selected.

\subsubsection{Material Models}

The definition of the steel material model is once again straightforward in these coupled thermo-mechanical analyses. The importance of the wood material model is amplified in a coupled thermal-mechanical analysis because the complexity of the material properties increases with the added reduction factors. The reduction factors applied according to the Eurocode vary in magnitude for elastic modulus in tension or compression and strength in tension, compression and shear (in varying material directions) (CEN 2004b).

\subsubsection{Steel Elements}

The steel elements were all assumed to be unaffected by the heating as the loading, support and anchorage plates were all unexposed to the fire and the cavity stayed below $100^{\circ} \mathrm{C}$. the thermal expansion of the tendons was considered negligible, the effect on the results from which is discussed in Section 3.6.3. Thus, the material properties used for all steel elements were the same as those described in Section 3.4.2.1.

\subsubsection{Wood Elasticity}

Similarly to the ambient analyses, the engineering constants model for elasticity was employed in the thermo-mechanical analysis, but with the moduli values decreased as a function of temperature. The stiffness reductions given in the Eurocode 5 Part 1-2 Annex $\mathrm{B}$ are given also as a function of stress state, varying from tension to compression (CEN 
2004b). Additionally, the modification factors are given only for the principal elastic modulus. Thus, it was assumed that the reduction factors could also be used for the other two material directions and all the shear moduli. The reduction factor for tension was used as the compression zone of the beam is largely unaffected by temperature, due to the top flange being insulated from the heat exposure. Table 3.8 gives the elastic engineering constants used as a function of temperature. Abaqus specifies that the minimum parameter reduction allowed is $1 / 100^{\text {th }}$ of the initial magnitude, so this requirement was used instead of the Eurocode specification of zero (CEN 2004b).

Table 3.8: Input parameters for engineering constants orthotropic elasticity as a function of temperature

\begin{tabular}{|l|l|l|l|l|l|l|l|l|l|}
\hline $\begin{array}{c}\mathbf{E}_{\mathbf{1}} \\
(\mathbf{M P a})\end{array}$ & $\begin{array}{c}\mathbf{E}_{\mathbf{2}} \\
(\mathbf{M P a})\end{array}$ & $\begin{array}{c}\mathbf{E}_{\mathbf{3}} \\
(\mathbf{M P a})\end{array}$ & $\mathbf{v}_{12}$ & $\boldsymbol{v}_{13}$ & $\mathbf{v}_{23}$ & $\begin{array}{c}\mathbf{G}_{\mathbf{1 2}} \\
(\mathbf{M P a})\end{array}$ & $\begin{array}{c}\mathbf{G}_{\mathbf{1 3}} \\
(\mathbf{M P a})\end{array}$ & $\begin{array}{c}\mathbf{G}_{\mathbf{2 3}} \\
(\mathbf{M P a})\end{array}$ & $\begin{array}{c}\text { Temperature } \\
\left({ }^{\circ} \mathbf{C}\right)\end{array}$ \\
\hline 13200 & 400 & 400 & 0.55 & 0.55 & 0.2 & 660 & 660 & 100 & 0 \\
\hline 13200 & 400 & 400 & 0.55 & 0.55 & 0.2 & 660 & 660 & 100 & 20 \\
\hline 6600 & 200 & 200 & 0.55 & 0.55 & 0.2 & 330 & 330 & 50 & 100 \\
\hline 132 & 4 & 4 & 0.55 & 0.55 & 0.2 & 6.6 & 6.6 & 1 & 300 \\
\hline
\end{tabular}

A preliminary analysis was run on model $\mathrm{C} 5$ with the purely elastic material model to observe the stresses that developed, in order to compare with the manufacturer material strengths and gauge the level of plasticity involved in the simulation. These results are discussed in the following section in order to select the yield criterion utilized in the Hill's Potential Function for plasticity.

\subsubsection{Wood Plasticity}

As the Hill's Potential Function for plasticity provided the most comparable results to experiments at ambient temperatures, this material model is the main focus of this section. 
Additionally, the Concrete Damaged Plasticity model is considered briefly to demonstrate the inapplicability in this scenario.

\section{Hill's Potential Function}

The Hill's plasticity model was employed first with only stiffness reduction in the orthotropic elasticity model according to Eurocode 5 Part 1-2 Annex B (CEN 2004b). The yield strengths were left as those used in the ambient model, however the tensile strength of $33 \mathrm{MPa}$ was used rather than the compressive strength of $38 \mathrm{MPa}$. This was done as the purely elastic analysis showed that the magnitude of stress within the model always remained below $33 \mathrm{MPa}$, and the region being heated was mainly the tension zone. The compressive strength perpendicular to grain was used rather than the tensile strength perpendicular to grain as the magnitude of tensile stress perpendicular to grain always remained below the strength of 1.4 MPa (Ardalany et al. 2011), however the compressive stress perpendicular to grain was above this value (but below the strength of $10 \mathrm{MPa}$ ). The manufacturer shear strength of 5.3 MPa was still used in all material directions. A summary of these values used without the reduction are shown in the first row of Table 3.9.

The mid-span deflection of model C5 (with no reduction in the Hill's potential yield strengths) compared to the experiment are shown in Figure 3.37. The experimental data for deflection during the fire exposure period begins at zero millimetres, although in reality the beam was post-tensioned and loaded prior to the fire exposure and would have had some non-zero deflection to begin with. If it is assumed that the data presented was zeroed for the beginning of the fire period, then the resulting deflections from the numerical simulation should also be normalized for this zeroed deflection. Performing this 
normalization results in reasonable agreement between the numerical and experimental deflections, as shown in Figure 3.37.

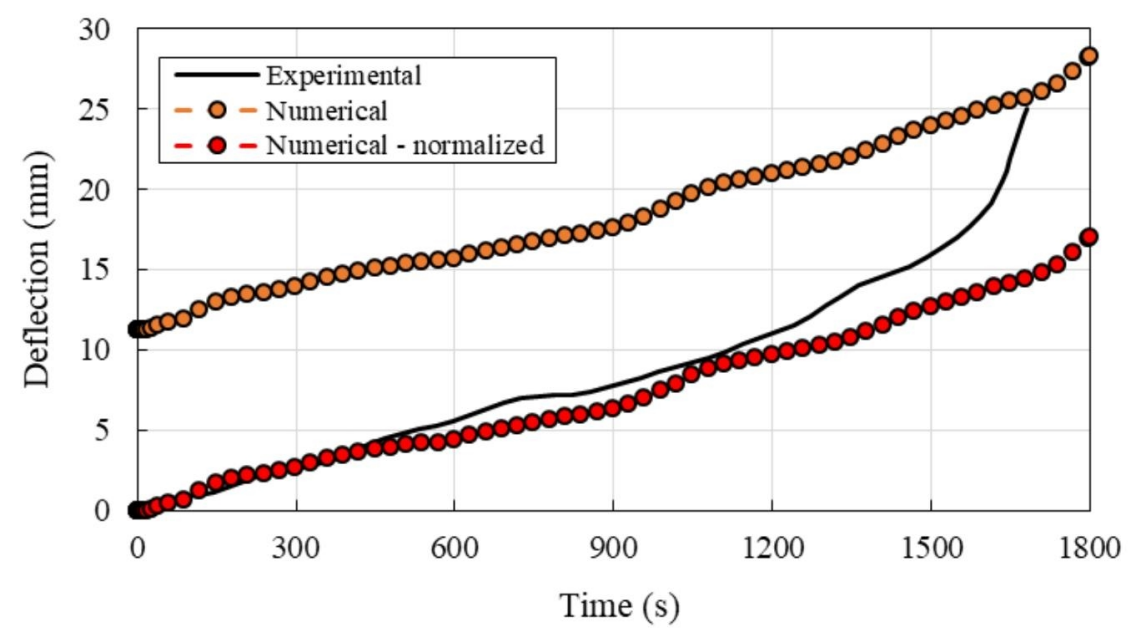

Figure 3.37: Results of Hill's plasticity model with reduced stiffness elastic orthotropic behaviour compared to experimental value for C5 (Costello 2013), with total and normalized deflections for the fire duration under constant loading (i.e. normalized does not include prior deflections from pre-fire loading and post-tensioning)

Although the yield stresses were not reduced in this simulation, the strength was still effectively reduced due to the stiffness reduction, which resulted in very small stresses in the heated timber areas. In order to check whether the stresses surpassed the actual reduced strength values, the stresses developed in this model versus the reduced strength in critical elements were plotted at a cross-section within the shear region. The reduced strengths were calculated from the average element temperatures from the thermal analysis, using the suggested reduction factors from Eurocode 5 Part 1-2 Annex B (CEN 2004b). The shear stress and reduced strength are plotted in Figure 3.38 for the elements described on the diagram. 

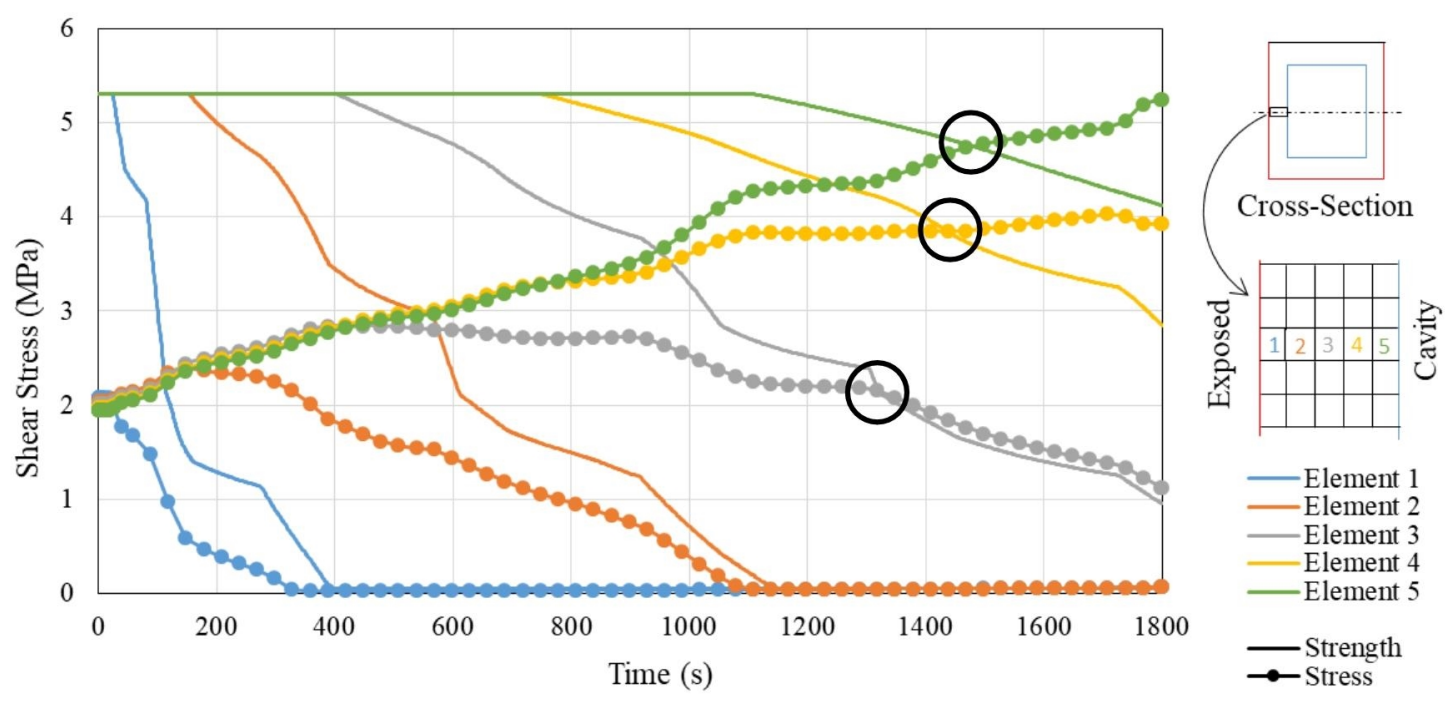

Figure 3.38: Shear stresses versus strength (reduced for average element temperature according to Eurocode 5 Part 1-2 Annex B) in web elements for a critical shear-region cross-section (CEN 2004b)

As can be seen in Figure 3.38, the shear stress in the outer elements reduces to zero by around 400 seconds (in element 1) and around 1100 seconds (in element 2), and the load is transferred to the inner elements, due to the reduction in stiffness of the outer heated elements. When plotted against the actual reduction in strength of the element due to temperatures from the thermal analysis, the stresses in outer elements 1 and 2 always lie within the strength envelope. However, the inner elements which have increased stresses due to load transfer do surpass their respective strengths further into the analysis, beginning at about 1300 seconds. This indicates that the strength should be reduced to get more realistic results, and also aligns with the deflection results at which time the numerical model departs from the experimental time-deflection behaviour seen in Figure 3.37.

A summary of the temperature-dependent reduced strength values and their corresponding ratios for use in the Hill's Potential Function for plasticity are shown in Table 3.9 and Table 3.10. As was previously stated, the tensile strength of $33 \mathrm{MPa}$ was used for the longitudinal 
yield stress, as no stresses in the elastic model surpassed this value. Additionally, the compressive zone remains close to ambient temperature as the top flange is unexposed to the fire, while the tensile zone has the most heat transfer and thus reduced strength. Therefore, it is justified to use the tensile strength and reduction factor of 0.65 from Eurocode 5 Part 1-2 Annex B for the longitudinal strength (CEN 2004b). Furthermore, the compression perpendicular to grain strength was again used as the tensile stresses perpendicular to grain were negligible. The reduction factor of 0.25 for compression perpendicular to grain was used (as is recommended in B3 sentence 2) (CEN 2004b). Thus, although the Hill's Potential Function in Abaqus does not allow for a differentiation between tension and compression, the use of one value over the other may be justified.

The manufacturer shear strength of 5.3 MPa was used in directions 1-2 and 1-3 with the Eurocode shear strength reduction factor of 0.4 , while the same shear strength was used in the rolling shear direction with a reduction factor of 0.25 (as is recommended in B3 sentence 3) (CEN 2004b).

Table 3.9: Strength values used in each of the material directions for calculation of the Hill's Potential Function ratios

\begin{tabular}{||l|l|l|l||l|l|l|l||l||}
\hline $\begin{array}{c}\boldsymbol{\sigma}_{\mathbf{0}} \\
(\mathbf{M P a})\end{array}$ & $\begin{array}{c}\boldsymbol{f}_{\mathbf{1 1}} \\
(\mathbf{M P a})\end{array}$ & $\begin{array}{c}\boldsymbol{f}_{\mathbf{2 2}} \\
(\mathbf{M P a})\end{array}$ & $\begin{array}{c}\boldsymbol{f}_{\mathbf{3 3}} \\
(\mathbf{M P a})\end{array}$ & $\begin{array}{c}\boldsymbol{\tau}_{\mathbf{0}} \\
(\mathbf{M P a})\end{array}$ & $\begin{array}{c}\boldsymbol{f}_{\mathbf{1 2}} \\
(\mathbf{M P a})\end{array}$ & $\begin{array}{c}\boldsymbol{f}_{\mathbf{1 3}} \\
(\mathbf{M P a})\end{array}$ & $\begin{array}{c}\boldsymbol{f}_{\mathbf{2 3}} \\
(\mathbf{M P a})\end{array}$ & $\begin{array}{c}\text { Temperature } \\
\left({ }^{\circ} \mathbf{C}\right)\end{array}$ \\
\hline 33 & 33 & 10 & 10 & 19.1 & 5.3 & 5.3 & 5.3 & 0 \\
\hline 33 & 33 & 10 & 10 & 19.1 & 5.3 & 5.3 & 5.3 & 20 \\
\hline 21.45 & 21.45 & 2.5 & 2.5 & 12.4 & 2.12 & 2.12 & 1.325 & 100 \\
\hline 0.33 & 0.33 & 0.1 & 0.1 & 0.191 & 0.053 & 0.053 & 0.053 & 300 \\
\hline
\end{tabular}

Table 3.10: Strength ratios used in Hill's Potential Function for plasticity

\begin{tabular}{|l|c|c|c|c|c|l|}
\hline $\boldsymbol{R}_{\mathbf{1 1}}$ & $\boldsymbol{R}_{\mathbf{2 2}}$ & $\boldsymbol{R}_{\mathbf{3 3}}$ & $\boldsymbol{R}_{\mathbf{1 2}}$ & $\boldsymbol{R}_{\mathbf{1 3}}$ & $\boldsymbol{R}_{\mathbf{2 3}}$ & $\begin{array}{l}\text { Temperature } \\
\left({ }^{\circ} \mathbf{C}\right)\end{array}$ \\
\hline 1 & 0.303 & 0.303 & 0.278 & 0.278 & 0.278 & 0 \\
\hline 1 & 0.303 & 0.303 & 0.278 & 0.278 & 0.278 & 20 \\
\hline 1 & 0.117 & 0.117 & 0.171 & 0.171 & 0.107 & 100 \\
\hline 1 & 0.303 & 0.303 & 0.278 & 0.278 & 0.278 & 300 \\
\hline
\end{tabular}


Similarly to the ambient material model, various constitutive models were implemented in order to approximate a more realistic relationship. However, due to the vast simplifications made in the material model, the largest being a symmetrical definition, the constitutive relationship did not hold much significance. Additionally, introducing multiple points in the strength versus plastic strain relationship with temperature dependency introduced complexity that Abaqus could not handle and the plasticity algorithm failed to converge.

\section{Concrete Damaged Plasticity}

The CDP material definition was also modelled in order to compare the results with the Hill's plasticity model. As the variation of the input parameters related to the concrete behaviour with temperature cannot be known for wood, they were left as independent of temperature, as were defined in Table 3.7. Once again, variations in these parameters were not found to influence the force-deflection behaviour of the simulations. The yield stresses were defined in tension and compression as functions of temperature. As the CDP model must be used with isotropic elasticity, the manufacturer specified elastic modulus was used with the tensile reduction factor from the Eurocode 5 Part 1-2 Annex B, as most of the compression zone remains near ambient temperature due to the protected top flange (CEN 2004b). The modulus and strength values used are outlined in Table 3.11.

Table 3.11: Elastic modulus and tension and compression strength values as a function of temperature for the CDP model

\begin{tabular}{|l|l|l|l|}
\hline $\begin{array}{c}\mathbf{E} \\
(\mathbf{M P a})\end{array}$ & $\begin{array}{c}\boldsymbol{f}_{\boldsymbol{t}} \\
(\mathbf{M P a})\end{array}$ & $\begin{array}{c}\boldsymbol{f}_{\boldsymbol{c}} \\
(\mathbf{M P a})\end{array}$ & $\begin{array}{c}\text { Temperature } \\
\left({ }^{\circ} \mathbf{C}\right)\end{array}$ \\
\hline 13200 & 33 & 38 & 0 \\
\hline 13200 & 33 & 38 & 20 \\
\hline 660 & 21.45 & 9.5 & 100 \\
\hline 132 & 0.33 & 0.38 & 300 \\
\hline
\end{tabular}




\subsubsection{Mesh Study}

A mesh sensitivity study was done on model C5 in order to determine grid independence. The mesh discussed in this section refers only to the elements used in the mechanical analysis, which differed from those used in the thermal analysis discussed in Section 3.5.3. The study was done with the Engineering Constants for elasticity, as described in Table 3.8, and the Hill's Potential Function for plasticity using yield strength ratios as a function of temperature, as described in Table 3.10. Three sizes of elements were compared. The mesh sizes are shown in Figure 3.39. The big mesh size consisted of $25 \mathrm{~mm}$ elements, but with a smaller $15 \mathrm{~mm}$ dimension through the web and flange thickness. That was done as three elements through the thickness of the webs and flanges was the minimum required for the simulation to successfully run. The small mesh size consisted of five elements through the web and flange thickness, thus making the nominal element dimensions in the cross-section $9 \mathrm{~mm}$. The longitudinal element size in the smaller mesh was $15 \mathrm{~mm}$. Finally, the medium mesh size was a hybrid between the big and small, with five elements through the web and flange thickness (9 mm dimension), but with the longer cross-sectional and longitudinal dimensions remaining at $25 \mathrm{~mm}$. Figure 3.40 shows the results of the mesh study. 


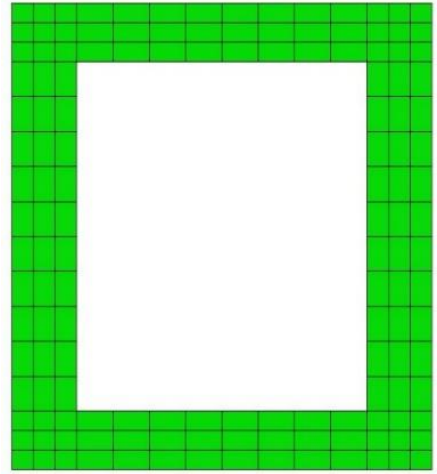

Big Mesh

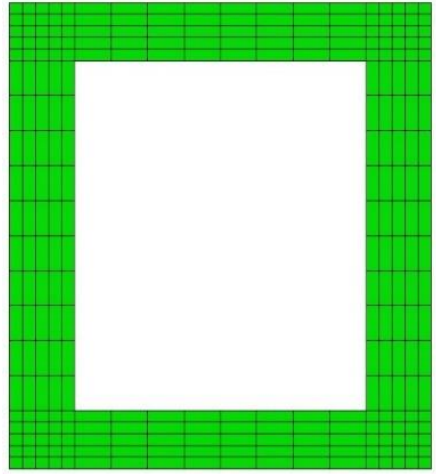

Medium Mesh

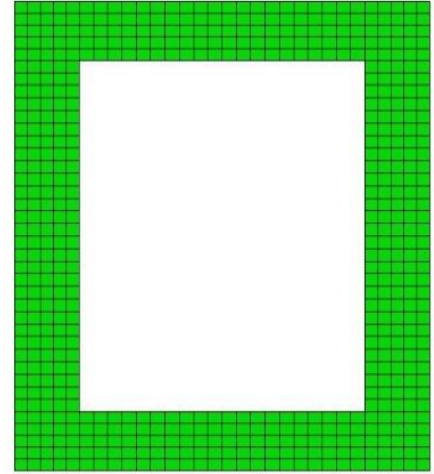

Small Mesh

Figure 3.39: Element sizes for mesh sensitivity study on model C5 where each web and flange element is 45 mm thick; dimensions for Beam 1 given in Figure 2.8

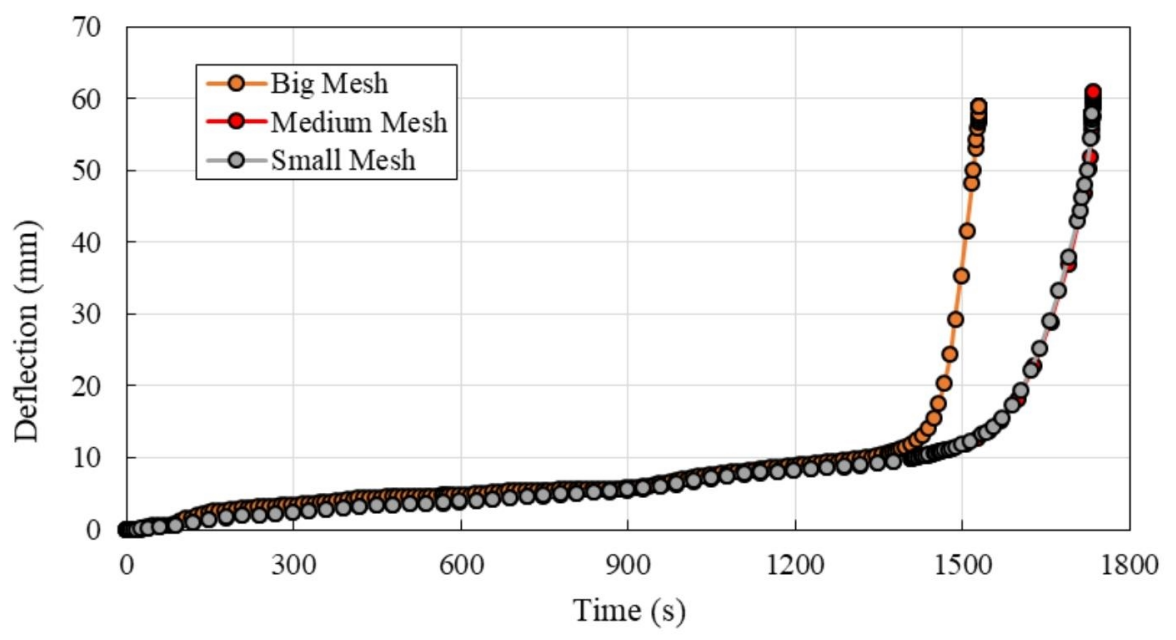

Figure 3.40: Mesh sensitivity study for model C5 with three sizes of mesh elements

As is shown in Figure 3.40, the results with the big mesh were severely different than the medium and small mesh sizes, in which the differences were negligible. The simulation with the bigger mesh exhibited similar behaviour prior to failure, but failed at an earlier time in the fire duration. This is likely due to the lower number of elements through the thickness of the webs and flanges, thus limiting the load transfer between unheated elements. Although the results were comparatively equal for the medium and small mesh 
sizes, the medium mesh size required only $15 \%$ of the computation time compared to the small mesh. Thus, the medium mesh size was used for the remainder of the simulations.

\subsubsection{Comparison of Results to Experiments}

The following results for the sequentially coupled thermo-mechanical analyses compare the time-deflection behaviour of the beams under constant load, with the heat exposure applied from the results of the heat transfer modelling discussed in Section 3.5. The CDP model was only compared for model C5 to demonstrate its inapplicability for predicting the behaviour of PT timber beams. The deflection results are zeroed at the beginning of the fire duration to match the experimental results, so any small deflections due to the pre-fire post-tensioning and loading were normalized.

\subsubsection{Model C5}

The behaviour of the beam in the numerical model for this experiment agreed quite well with the experimental results. A comparison between the mid-span deflections during the fire exposure of the model and the experiment is shown in Figure 3.41. The failure time of the numerical model due to runaway deflections was 29 minutes, identical to the recorded experimental failure time, at which time large deflections and a loss of load occurred in the furnace (Costello 2013). This, along with the comparison between failure modes in Figure 3.42 , indicates that the behaviour and failure mechanism predicted by the model was accurate. It is expected that if the experiment was continued and data was collected past the point where the data ends in Figure 3.41, runaway deflections similar to the Hill's Potential Function numerical model results would be seen. 


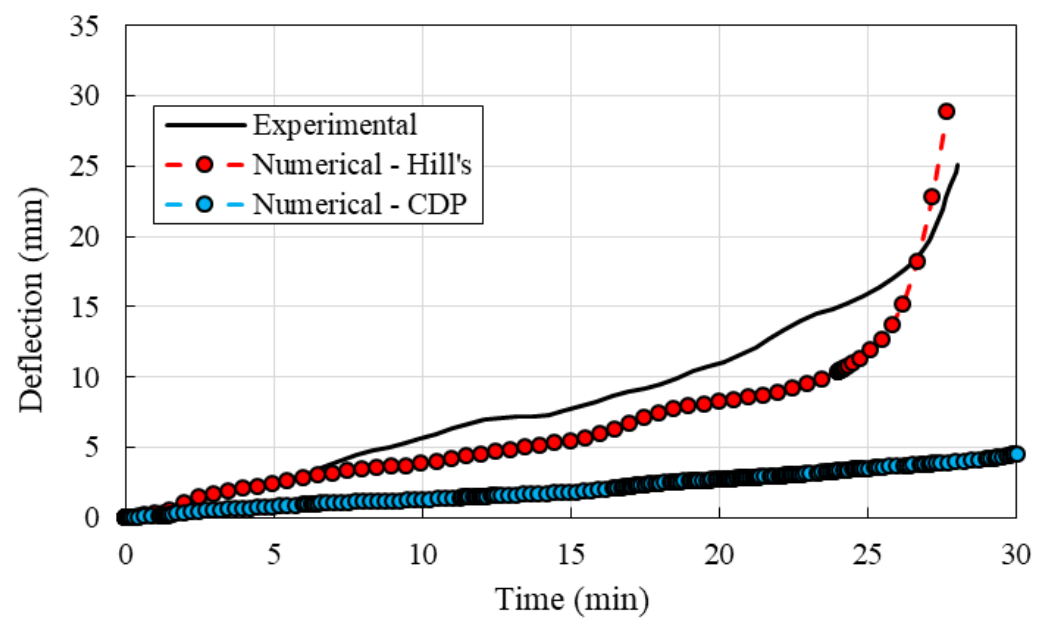

Figure 3.41: Vertical displacement versus time into heat exposure for C5 is shown compared to the numerical model results from the Hill's Potential Function and the CDP models (Costello 2013)

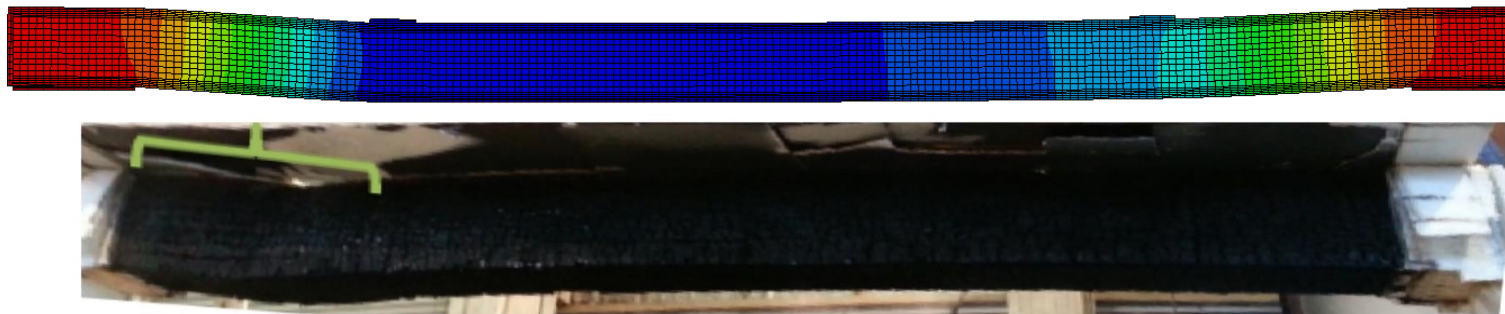

Figure 3.42: Comparison of failure modes in numerical model (above) and experimental beam (below) where a shear region failure between the support outside the furnace and the load application point can be seen (Costello 2013)

The longitudinal compressive stresses increased slightly and reached the tensile strength value used of $33 \mathrm{MPa}$. This indicates that the model may have predicted some plasticity in the compressive regions before the manufacturer yield strength of $38 \mathrm{MPa}$. However, based on the Eurocode 5 Part 1-2 Annex B strength reductions, wood in compression loses its strength with temperature more dramatically than wood in tension, so this reduction in strength may have been okay (CEN 2004b).

Also from Figure 3.41, it can be seen that the CDP model does not predict the experimental behaviour of the beam at all. Although strengths may be input separately for compression 
and tension, the isotropic nature of the material model results in a stiffness that is much too high. The model did not nearly predict the deflections or failure mechanisms seen in the experiment. It was thus concluded that the CDP model is not suitable to represent the behaviour of PT timber beams.

The internal cavity temperature was assumed conservatively to be the uniform temperature of the tendon, and the thermal expansion was calculated to ensure the stresses that arose would be negligible (see Figure 3.43). The thermal expansion strains were calculated using the prestressing steel thermal elongation equation from Eurocode 2, Part 1-2 (CEN, 2004c), shown in Equation 3.7.

$$
\varepsilon_{t}=-2.016 \times 10^{-4}+10^{-5} \mathrm{~T}+0.4 \times 10^{-8} T^{2} \text { for } 20^{\circ} \mathrm{C} \leq T \leq 1200^{\circ} \mathrm{C}
$$

Equation 3.7

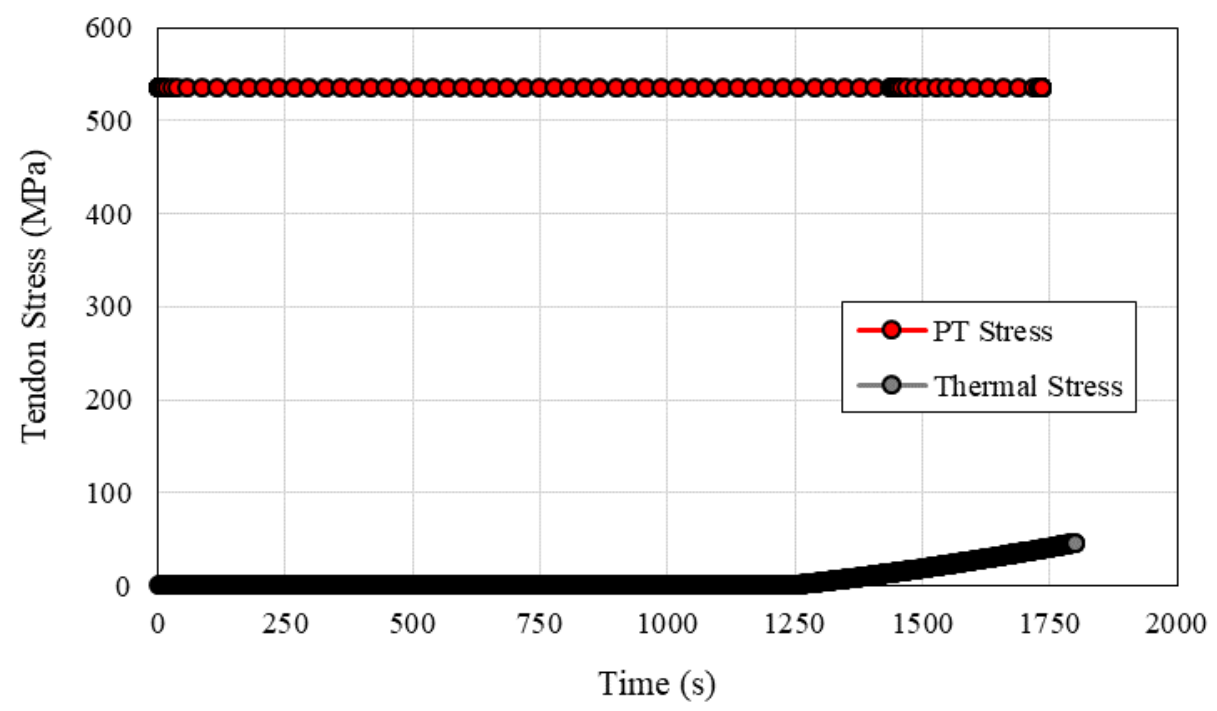

Figure 3.43: Stress due to thermal expansion of the tendons versus the post-tensioning stress in the C5 numerical model

As is shown in Figure 3.43, the stress due to thermal expansion would be negligible compared to the stress from PT. Although no tendon force data was given in Costello's 
furnace test results, the model tendon force remained at $107 \mathrm{kN}$ for the entire simulation corresponding exactly with the PT force introduced in the experiment.

\subsubsection{Model S6}

The thermal-mechanical results for Spellman's tests are once again presented as deflection versus time data. In Spellman's experiments, the deflection was measured at the load actuator, and thus recorded the average displacement of the two loading points rather than the mid-span deflection. Accordingly, the deflection results from the numerical model were reported as the average vertical displacement of the central nodes on each loading plate. A comparison between the numerical and experimental results is shown in Figure 3.44. The failure time in the model due to runaway deflections was 66 minutes, two minutes later than the experimental failure time of 64 minutes from the same mechanism. It can be seen, however, that the failure of the beam in the simulation was much more abrupt than the more gradual failure of the experiment. The simulation deflection remained near zero for the majority of the simulation as the PT tendon force largely counteracted the vertical load.

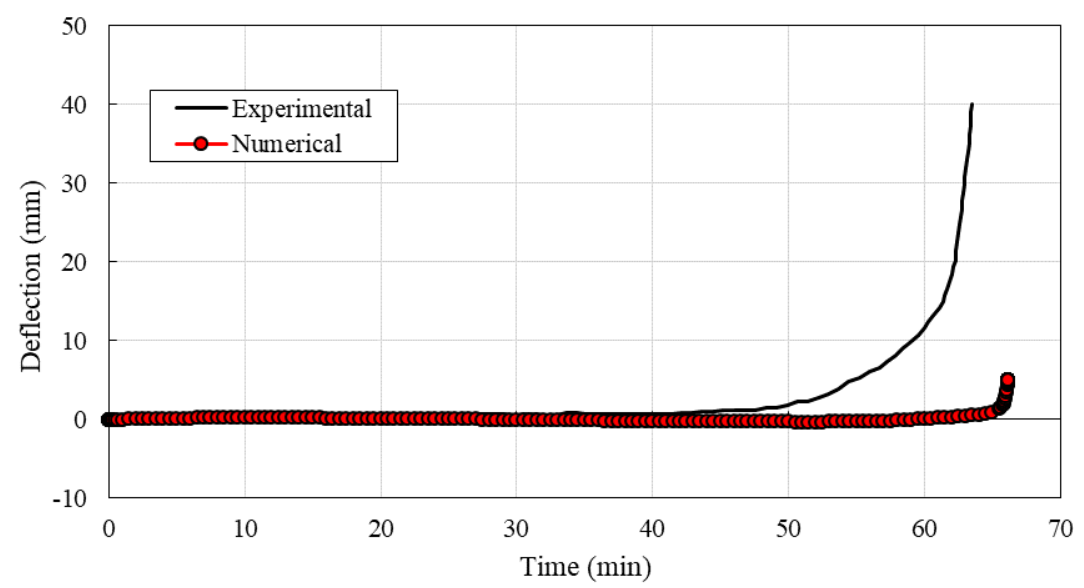

Figure 3.44: Vertical displacement versus time into heat exposure for S6 is shown compared to the numerical model results from the Hill's Potential Function model (Spellman 2012) 
Much of the wood was degraded and missing from the beam once it was able to be taken out of the furnace and cooled, so it was difficult to compare the failure modes of the beams. In the deformed shape of the model beam at the end of the simulation, local buckling can be seen near the support, around where the heat exposure ended, shown in Figure 3.45. Compared to the image of the beam from after the experiment, although not much can be told of the failure, some slight deformation in the same spot near the support, where the beam left the furnace, can be seen. The buckling failure in the model compared to the deflection seen in the experiment photo could be the reason that the simulation failure was much more abrupt than the experiment. Additionally, cracking was not accounted for in the model which would have caused the beam to lose stiffness, indicating that in reality the beam would have seen more deflection than in the model.
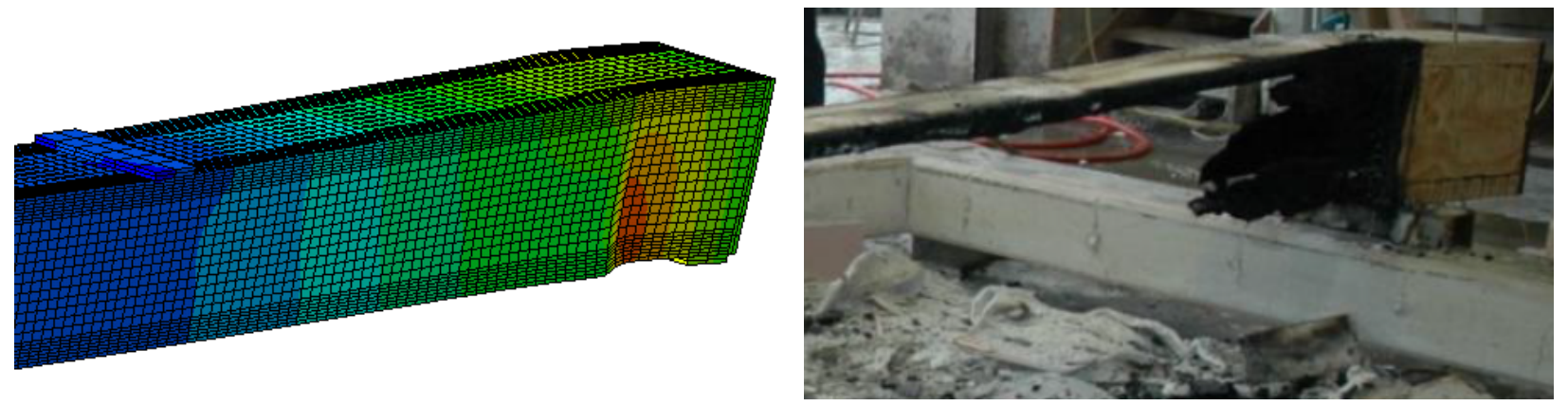

Figure 3.45: Comparison between model failure mode (left) and beam after furnace test (right) where slight buckling at the furnace edge interface can be seen (Spellman 2012)

A look at the average tendon PT force in the numerical model in Figure 3.46 shows that the model behaviour, similarly to the deflection, was maintained more than in the experiment until the end where an abrupt failure occurred. This small discrepancy could be due to tendon relaxation from localized heat penetration or overall thermal expansion. A comparison between the thermal expansion stresses that would have occurred if considered are shown in Figure 3.47. 


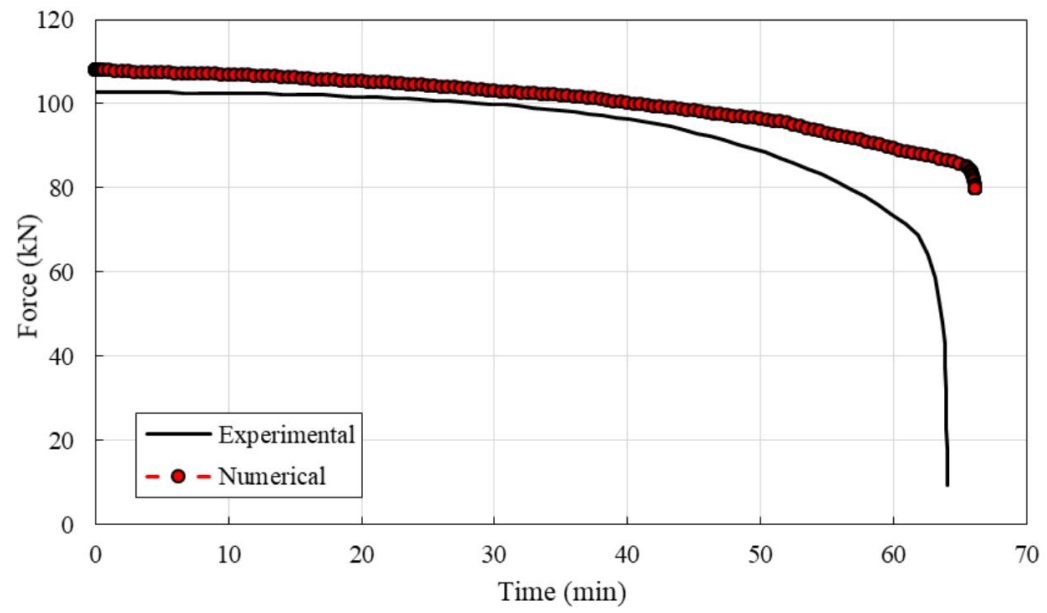

Figure 3.46: Comparison of S6 numerical model results to experimental data for the average tendon force versus time into heat exposure (Spellman 2012)

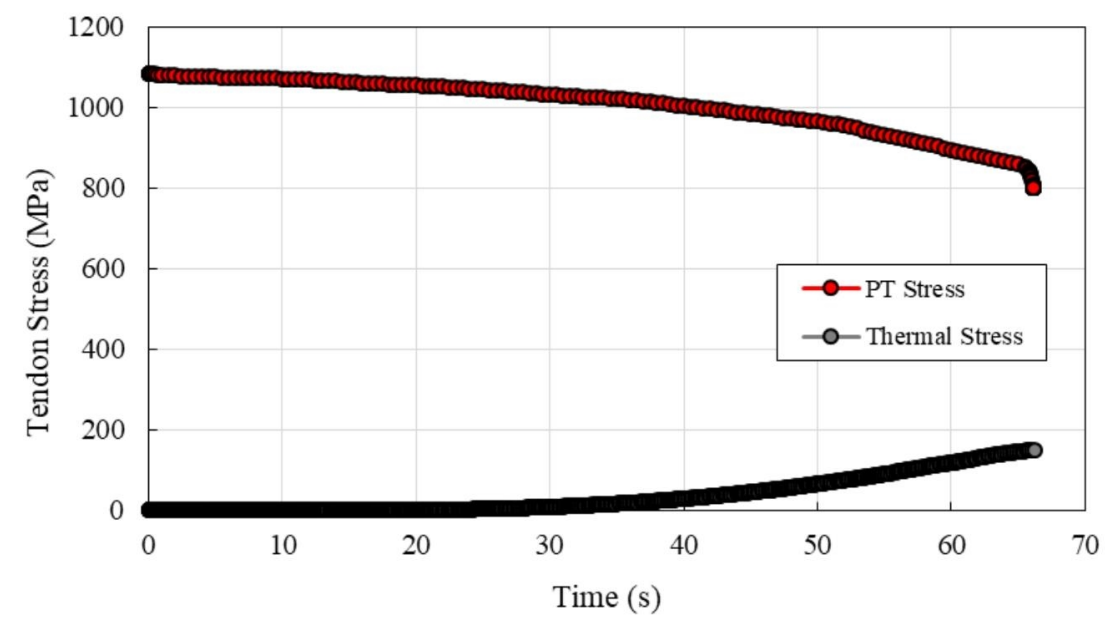

Figure 3.47: Stress due to thermal expansion of the tendons versus the post-tensioning stress in the S6 numerical model

It is seen that if the tendon was considered to be at the temperature of the interior surface of the timber in the model, the thermal expansion stresses (calculated as per Equation 3.7) were higher than that of model $\mathrm{C} 5$. This may have resulted in some minor tendon relaxation which could account for the discrepancies between the numerical and experimental results. 


\subsubsection{Model S7}

A comparison between the numerical model and experimental deflections for Spellman's Beam $\mathrm{C}$ is shown in Figure 3.48 where the displacements are measured in the same way as Model S6. The simulation failed at 53 minutes due to runaway deflections, very similarly to the experimental results but three minutes earlier, as the experiment failed at 56 minutes. The deflections followed very accurately until around 40 minutes at which point, in the same way as Model S6, the experiment began to fail more gradually than the numerical model which had a slightly more abrupt failure. The failure mechanism in the model was due to shear and bending, while the experiment showed a clear failure due to web cracks and buckling in the shear region.

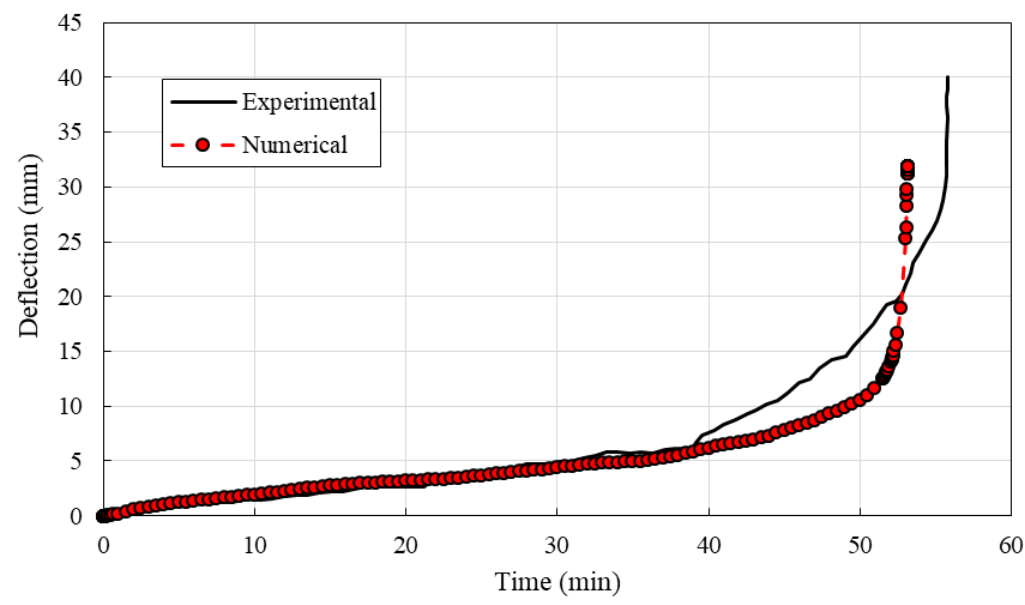

Figure 3.48: Vertical displacement versus time into heat exposure for $\mathrm{S} 7$ is shown compared to the numerical model results from the Hill's Potential Function model (Spellman 2012)

The tendon force was investigated to ensure the model can accurately predict tendon relaxation due to deflections without accounting for heat transfer to the steel tendon. A comparison between the numerical average tendon force and the experimental average tendon force is shown in Figure 3.49. The figure shows that the model results are in very 
good agreement with the averaged measured force from the experimental data, until failure about three minutes prior to the experiment (lining up with the deflection data shown in Figure 3.48). This indicates that the assumption of the steel tendons being unaffected by temperature is acceptable. The estimated tendon thermal expansion stresses (from Equation 3.7) which may have arose are compared to the actual tendon stresses from the model in Figure 3.50 .

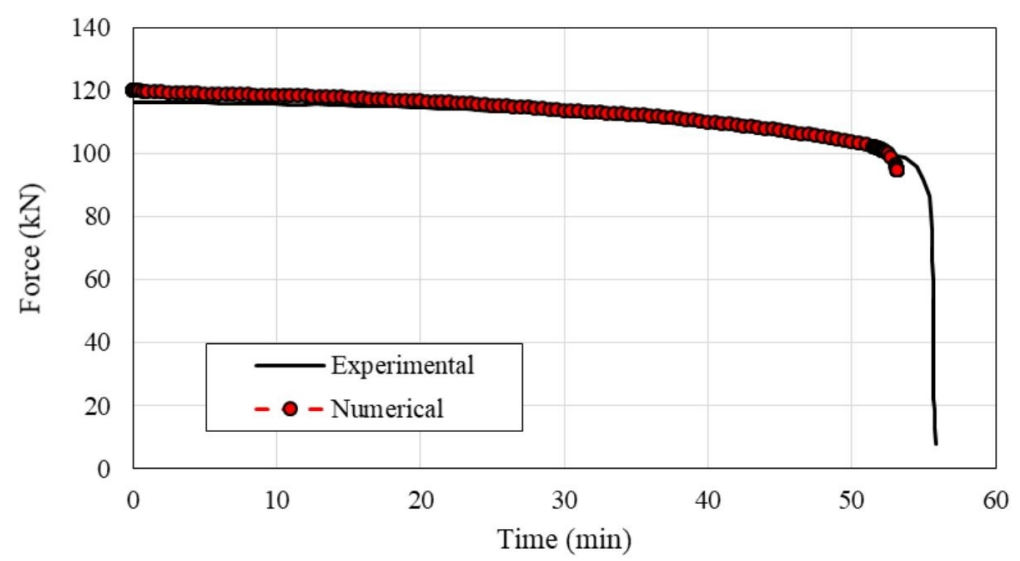

Figure 3.49: Comparison of the $S 7$ numerical model results to experimental data for the average tendon force versus time into heat exposure (Spellman 2012)

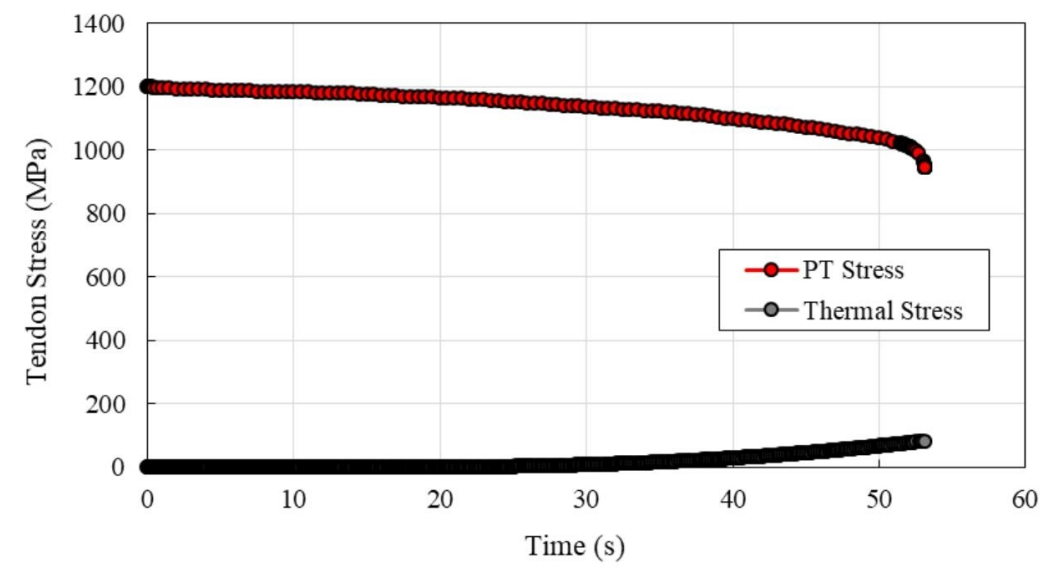

Figure 3.50: Stress due to thermal expansion of the tendons versus the post-tensioning stress in the $S 7$ numerical model 
Figure 3.49 and Figure 3.50 indicate that the relatively small thermal stresses developed in the tendon would be negligible on the performance of the beam.

\subsection{Recommendations and Conclusions}

A numerical model was successfully developed and verified for PT timber beams in both ambient and fire conditions using Abaqus finite element modelling software. The results of this study have shown good approximations of the beam behaviours which have not previously been achieved. Additionally, the model development presented herein offered a detailed review of available material models and a clear path for future timber modelling tool advancements. Abaqus was a good platform for the model development for research purposes, in order to investigate the merits and downfalls of commercially available material model formulations. The development of a model specific to this application in a more accessible format would be desirable so practitioners may have the ability to design and optimize the structure for approval purposes, without the requirement of a costly FEM package. In lieu of this model, a step-by-step procedure for creating an FEM model of the type developed herein would be beneficial for practitioners unexperienced in the software.

In general, the results have indicated that a model which includes the orthotropic nature of wood in the material model is more significant to produce good results than the need to differentiate between tensile and compressive strengths. However, for the most realistic and reliable results, a model where both of these phenomena may be captured simultaneously is desired. More detailed conclusions and recommendations for each model type are given in the following sections. 


\subsubsection{Ambient Model}

The numerical model results for the ambient beam tests showed decent correlation with the experimental data, although the exact behaviour was not predicted particularly well. A summary of the failure loads and deflection behaviour of each test is shown in Table 3.12. From there it can be seen that the failure loads for most of the beams were predicted well (within $10 \%$ of the actual failure load), except for model $\mathrm{C} 2$ for Costello's fourth beam test. Additionally, in most cases, the deflection was highly overestimated compared to the experimental observations. This could be an issue with the model allowing excessive, unrealistic deflections before which the test would be stopped in reality. The simulations are load controlled which means that the same failure behaviour with a decrease of load with increased deflections could not be observed. However, when attempted with strokecontrolled analyses, the same behaviour was seen where the loads did not decrease before the failure of the simulation.

Table 3.12: Comparison between numerical model and experimental failure loads and deflections

\begin{tabular}{|c|c|c|c|c|c|}
\hline Model & $\begin{array}{c}\text { Experiment } \\
\text { Failure } \\
\text { Load (kN) }\end{array}$ & $\begin{array}{c}\text { Model } \\
\text { Failure } \\
\text { Load } \\
\text { (kN) }\end{array}$ & Difference & $\begin{array}{c}\text { Experiment } \\
\text { Failure } \\
\text { Deflection } \\
\text { (mm) }\end{array}$ & $\begin{array}{c}\text { Model Failure } \\
\text { Deflection } \\
\text { (mm) }\end{array}$ \\
\hline C1 & 473 & 480 & $1.5 \%$ & 24 & 87 \\
C2 & 147 & 220 & $50 \%$ & 16 & $400+$ \\
C3 & 515 & 465 & $-9.7 \%$ & 21 & 46 \\
C4 & 138 & 145 & $5.1 \%$ & 22 & 36 \\
\hline
\end{tabular}

In some cases, the tensile stresses in the models surpassed their specified strengths in the plasticity model where they did not in the purely elastic model. This is due to load redistribution after the failure of compression regions which reached the manufacturer's 
compressive yield strength. This is a shortcoming of the Hill's Potential Function model for plasticity in which only one strength for each direction may be defined, independent of tensile or compressive stress states. In the future, for ambient modelling, it is shown that it is important for the model to include both tensile and compressive strength values, or some sort of cracking model in order to deal with the lower tensile strengths and failure modes.

\subsubsection{Heat Transfer Model}

The heat transfer models showed good results when compared with the available experimental data. The importance of having an accurate estimate of material density and moisture content was apparent. Even when the temperature results did not totally match the experimental data for the simulations, the char depths were predicted quite well. A summary of the predicted char depths compared to the experimentally observed char is shown in Table 3.13. The predicted char depths were always smaller than the measured char which could be due to additional charring in the cooling or extinction phases of the furnace tests which are not accounted for in the model. This indicated that the heat transfer model results were accurate and the discrepancies may be due to placement errors between the nodes and the actual thermocouple locations.

Table 3.13: Comparison between numerical model and experimental char depths

\begin{tabular}{|c|c|c|c|}
\hline Model & $\begin{array}{c}\text { Experiment Char Depth } \\
(\mathbf{m m})\end{array}$ & $\begin{array}{c}\text { Model Char Depth } \\
(\mathbf{m m})\end{array}$ & $\begin{array}{c}\text { Difference } \\
(\mathbf{m m})\end{array}$ \\
\hline C5 & 25 & 20 & -5 \\
S6 & 47.5 & 44 & -3.5 \\
S7 & 40 & 39 & -1 \\
\hline
\end{tabular}




\subsubsection{Thermal-Mechanical Model}

The numerical model results for all three scenarios showed impressive accuracy when compared to the experimental data, which surpasses previous attempts at modelling PT timber beams in fire, and wooden beams in general. The Hill's Potential Function for the plasticity model of wood proved to be much more suited to this modelling scenario than others, including the Concrete Damaged Plasticity model. Typically, the numerical model followed the deflection behavior of the experiment quite well for the majority of the simulation. The model failure modes were comparable but were typically more abrupt than the experiment, with the failure times always within a few minutes of the actual times. A summary of the failure time comparisons between the numerical model results and the experiments is shown in Table 3.14 .

Table 3.14: Comparison between numerical model and experimental failure times

\begin{tabular}{|c|c|c|c|}
\hline Model & $\begin{array}{c}\text { Experiment Failure Time } \\
\text { (min) }\end{array}$ & $\begin{array}{c}\text { Model Failure Time } \\
(\mathbf{m i n})\end{array}$ & $\begin{array}{c}\text { Difference } \\
(\mathbf{m i n})\end{array}$ \\
\hline C5 & 29 & 29 & 0 \\
S6 & 64 & 66 & +2 \\
S7 & 56 & 53 & -3 \\
\hline
\end{tabular}

The tendon force comparisons were in good agreement, indicating that the assumption of the tendons being unaffected by the temperatures they were exposed to was acceptable. For the most part, the models were able to reasonably predict the failure times and modes of the PT timber beams exposed to fire conditions. 


\section{Chapter 4: Timber Adhesive Performance after Fire}

\section{Damage}

\subsection{Introduction}

Over recent years, new engineered timber products, construction methods and systems have increasingly spread over North America. With the advancements made in the timber products industry in the last several decades, innovative systems and structures have been rapidly developed and erected. Since engineered timber products were invented, there have been numerous standards developed to ensure their performance is at least equivalent to solid-sawn timber, especially at high temperatures. These standards are reviewed in detail in Section 2.1.4. However, even these stringent standards lack in one area - the materials are not subjected to the practical conditions that they may actually encounter in application, in the event of a critical fire scenario. A deeper understanding of the performance in these critical situations is imperative to safe structural fire engineering analysis and design. This is especially true in such novel and complex systems where engineered timber products may be used in a new way, such as post-tensioned timber. Having knowledge of the material behaviour itself (as opposed to only the system as a whole) is imperative in the efficient and safe design and modelling of new systems. The findings of the authors which has led to this experimentation on engineered timber adhesives is detailed in Section 4.2.

\subsection{Background and Motivation}

In order to implement new, complex timber structures, it is imperative to have an understanding of the material behaviour in a fire and failure mechanisms that may be unique to the novel system. Additionally, resilience of fire-damaged structures post-fire 
events is increasingly becoming of concern. For this reason, an experimental programme was undertaken by the author to research the underlying mechanics of the failure of firedamaged engineered timber, specifically in a built-up box configuration, which was published in (Quiquero et al. 2016) and (Quiquero and Gales 2016)i. The test series included axial compression of glulam coupons and four-point loading of short glulam beam sections - the series is summarized in Table 4.1. The glulam specimens were provided by Nordic Structures (materials discussed further in Section 4.3) and the box-beams were built up in the lab from the provided smaller sections. The box beam sections were tested at two orientations, one with the flanges extending the full width of the box and the other $\left(90^{\circ}\right)$ with the webs extending the full depth of the box. A group of the samples were burned in a furnace in a one hour standard fire at the Carleton/NRC fire testing lab, and allowed to burnout after fuel supply was halted for an additional hour. Following this period any continuing smouldering was extinguished with minimal water drawn from a hand-pump and the samples were allowed to cool naturally. They were then transported to the Carleton structures lab and tested at a later date so the failures could be viewed and captured in detail. The specimens were tested mechanically after cooling rather than during the fire exposure to ensure accurate and detailed documentation of the specimen deformations and behaviour could be recorded. Of the samples that were not burnt, some were left as original control specimens and a portion were manually altered to carve off the corresponding char depth to the burnt specimens.

\footnotetext{
i These conference papers were written prior to the author commencing the MASc degree. The study is summarized herein as motivation for this thesis but is outside the boundary of this thesis to explain in full.
} 
Table 4.1: Summary of Previous Experiments

\begin{tabular}{|c|c|c|}
\hline Specimen & Modifications & Details \\
\hline A1-1 & Burned & \multirow{3}{*}{$181 \mathrm{~mm}$ x $181 \mathrm{~mm}$ Coupon } \\
\hline A1-2 & Manually reduced & \\
\hline A1-3 & Unaltered & \\
\hline A2-1 & Burned & \multirow{3}{*}{$228 \mathrm{~mm}$ x $228 \mathrm{~mm}$ Coupon } \\
\hline A2-2 & Manually reduced & \\
\hline A2-3 & Unaltered & \\
\hline B1-1 & Burned & \multirow{4}{*}{ Solid Beam } \\
\hline B1-2 & Burned & \\
\hline B1-3 & Manually reduced & \\
\hline B1-4 & Unaltered & \\
\hline B2-5 & Burned & \multirow{5}{*}{ Box Beam } \\
\hline B2-6 & Burned & \\
\hline B2-7 & Burned & \\
\hline B2-8 & Unaltered & \\
\hline B2-9 & Unaltered & \\
\hline B2-10 & Burned & \multirow{4}{*}{ Box Beam, $90^{\circ}$} \\
\hline B2-11 & Burned & \\
\hline B2-12 & Manually reduced & \\
\hline B2-13 & Unaltered & \\
\hline
\end{tabular}

Specimens were carved to remove the char depth recorded plus a zero strength layer in order to investigate whether the assumption that the cross-section below the pyrolysis zone has full strength. This procedure was similar to what was done on full-scale box beams by Costello (2013). The code-recommended standard charring rate of $0.7 \mathrm{~mm} / \mathrm{min}$ plus a zerostrength layer of $7 \mathrm{~mm}$ would predict a depth of $49 \mathrm{~mm}$ for a one hour standard fire exposure. The average char depth recorded (plus zero-strength layer) for the exposed beam surfaces was $55 \mathrm{~mm}$. However, the beams were left to cool in the furnace for an additional hour beyond the one hour standard fire, during which smouldering and some flaming was still observed, so the char depth would be expected to be much larger than $49 \mathrm{~mm}$ and thus, these code predictions appear to remain conservative for their intention. 
In all cases in which a corresponding specimen was carved to match a burnt specimen, the manually reduced test always had a significantly higher peak load than the burnt counterpart. For example, a general comparison between several of the beam tests is shown in Figure 4.1, where the percent of peak loads causing failure compared to the ambient, unaltered peak load is shown. The burned specimen failed initially around the same displacement as the others with a large reduction in load, but the load was redistributed and was able to be carried by the webs for an extended period shown by the dashed portion (likely due to the short length of the specimen so that slenderness was not an issue).

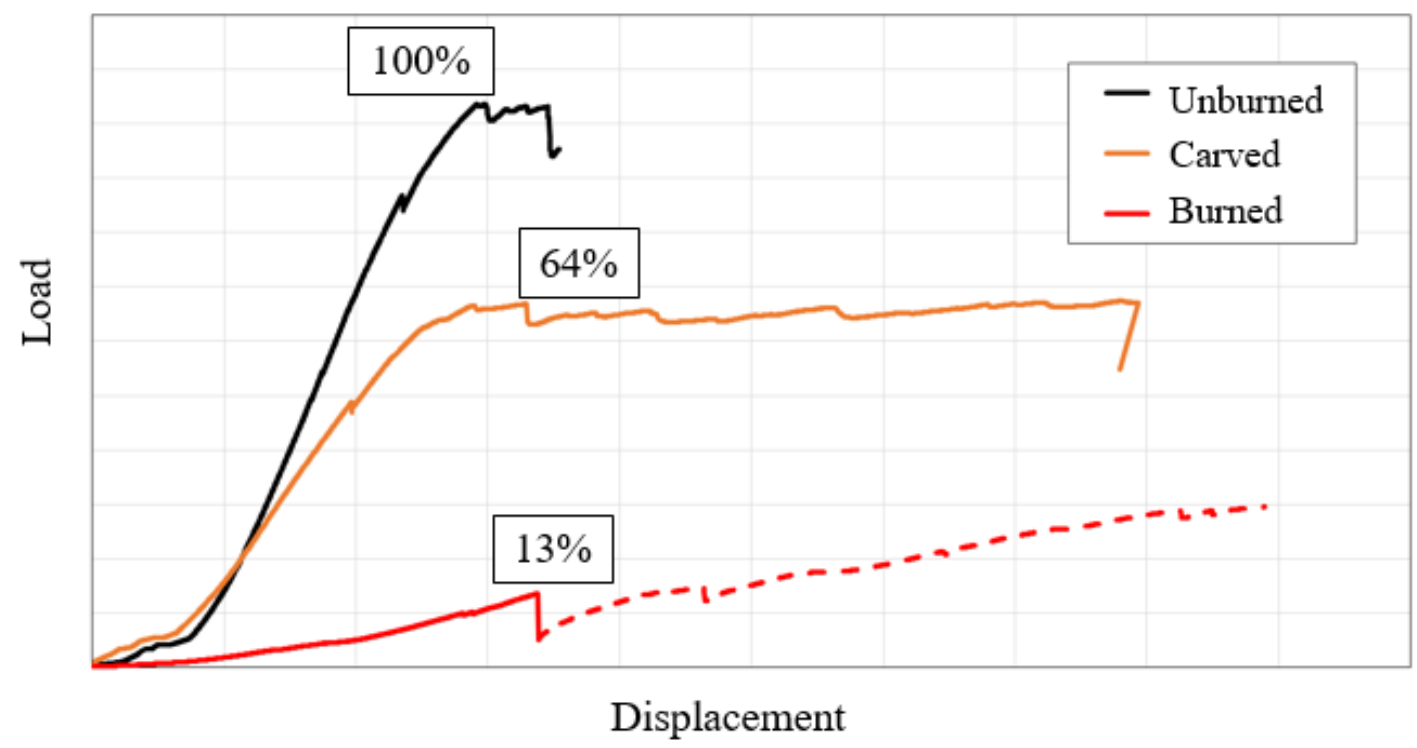

Figure 4.1: Glulam box-beam four-point loading tests comparative load versus displacement data (Quiquero et al. 2016)

This indicated that there was some other factor contributing to the failure of the specimens other than the loss of cross-sectional area. The adhesive in the engineered timber could be affected by the extreme heating and the charring encroaching on glue-lines, causing it to have a reduced strength, which has been identified as a research need in the recently 
released ASCE Manual of Practice (LaMalva et al. 2018) ii. The manufacturer adhesive used in the glulam specimens was a proprietary PUR adhesive, and a PRF adhesive was used in the lab to build up the box-beam sections (more background on these adhesives was given in Section 2.1.4). In order to test this hypothesis, a test programme was planned to specifically test the shear performance of the adhesive bonds which had been encroached on by extreme heating and charring for various heating durations. The shear strength of adhesive lines is often used as a performance criterion as it is a common interfacial stress state in structural members in practice (Pizzo et al. 2003). Additionally, a common failure mechanism seen in PT timber beams in furnace tests was shear failure in the webs around stress concentrations at the flange-web connection areas.

\subsection{Experimental Methodology}

In order to test the hypothesis for the research study, an experimental program was developed in which over 40 samples of Glulam adhesive lines were tested under various conditions. Specimens were prepared in the lab from Glulam products made of sprucepine-fir (S-P-F) species, primarily of Black Spruce, which is the most commonly used species group available in Canada. The timber was provided by Nordic Structures (Montréal, Québec) of stress grade 24F-ES/NPG. The manufacturer specified shear strength was 2.5 MPa and axially-loaded elastic modulus was $13100 \mathrm{MPa}$ (Nordic Wood Structures 2015). Glulam blocks were prepared with a thickness of one laminate (40 mm), and with an area of $100 \mathrm{~mm}$ by $100 \mathrm{~mm}$. The specimens used differed from the standard shear test specimens described in Section 2.1.4 corresponding to the required sample size

ii The previous study described herein was reviewed by an expert panel in 2017 during the development of this Manual of Practice and lead to the identification of this research need. 
for the cone calorimeter apparatus (see below for description). The blocks were cut from the larger Glulam billets, ensuring that an adhesive line was located along the centreline of the sample for shear testing. The samples were cut around manufacturer made glue lines which used a proprietary PUR adhesive, and around lab-prepared PRF adhesive lines. The PRF adhesive was supplied by Hexion in two parts: Cascophen LT-5210J Phenol Formaldehyde Resorcinol resin and Cascoset FM-6210 Paraformaldehyde catalyst.

A list of all the samples tested is shown in Table 4.2. In an initial round of tests, two replicates of each test were done (except for the PRF samples which were lower in availability, and only one of each was initially done at $30 \mathrm{~kW} / \mathrm{m}^{2}$ and at ambient). After this testing, it was clear that more repeated tests should be done to somewhat improve the variability. The most variable tests were identified and additional specimens were tested with those parameters, leading to more replicate samples being done for some parameters than others. Only one sample was tested for the PRF adhesive at ambient due to limited availability, however all the unheated samples showed repeatability as the failure was in the wood rather than the adhesive (which is to be expected due to current adhesive standards). The failure stresses were all similar and were well above the manufacturer specified shear strength of the Glulam of 2.5 MPa. More details on the experimental methodology are given in the following sections. 
Table 4.2: Summary of the number of samples tested for each adhesive type, incident heat flux exposure and heating duration

\begin{tabular}{|c|c|c|c|c|}
\hline \multirow{2}{*}{$\begin{array}{c}\text { Heating } \\
\text { Duration } \\
\text { (min) }\end{array}$} & \multicolumn{2}{|c|}{$\begin{array}{c}\text { Number of PUR samples with } \\
\text { each heat flux exposure }\end{array}$} & \multicolumn{2}{|c|}{$\begin{array}{c}\text { Number of PRF samples with } \\
\text { each heat flux exposure }\end{array}$} \\
\cline { 2 - 5 } & $30 \mathrm{~kW} / \mathrm{m}^{2}$ & $50 \mathrm{~kW} / \mathrm{m}^{2}$ & $30 \mathrm{~kW} / \mathrm{m}^{2}$ & $50 \mathrm{~kW} / \mathrm{m}^{2}$ \\
\hline 0 & \multicolumn{2}{|c|}{2} & \multicolumn{2}{|c|}{1} \\
\hline 3 & 3 & 2 & 3 & 2 \\
\hline 6 & 3 & 2 & 2 & 2 \\
\hline 10 & 4 & 4 & 2 & 3 \\
\hline 15 & 4 & 3 & 2 & 2 \\
\hline
\end{tabular}

\subsubsection{Cone Calorimeter Heating}

A cone calorimeter was used as a heat source with a constant incident heat flux which is depicted in Figure 4.2. The apparatus consists of a conical shaped heating element which is calibrated to ensure an accurate radiant heat flux is applied. The sample is placed at a fixed distance from the heating coil and a shield is placed around the specimen to minimize convective heat transfer. The shield also acts as a barrier for the combustion gases to be funnelled into the oxygen calorimeter which computes the heat release rate of the burning sample.

The plate which the specimen rests on is also a scale and the mass loss rate of the specimen is recorded throughout the burn duration. The ignition point of each sample is recorded on the calorimeter data collection software. Several other indicators are measured by the apparatus and are exported, including smoke generation, carbon monoxide and carbon dioxide yields. 

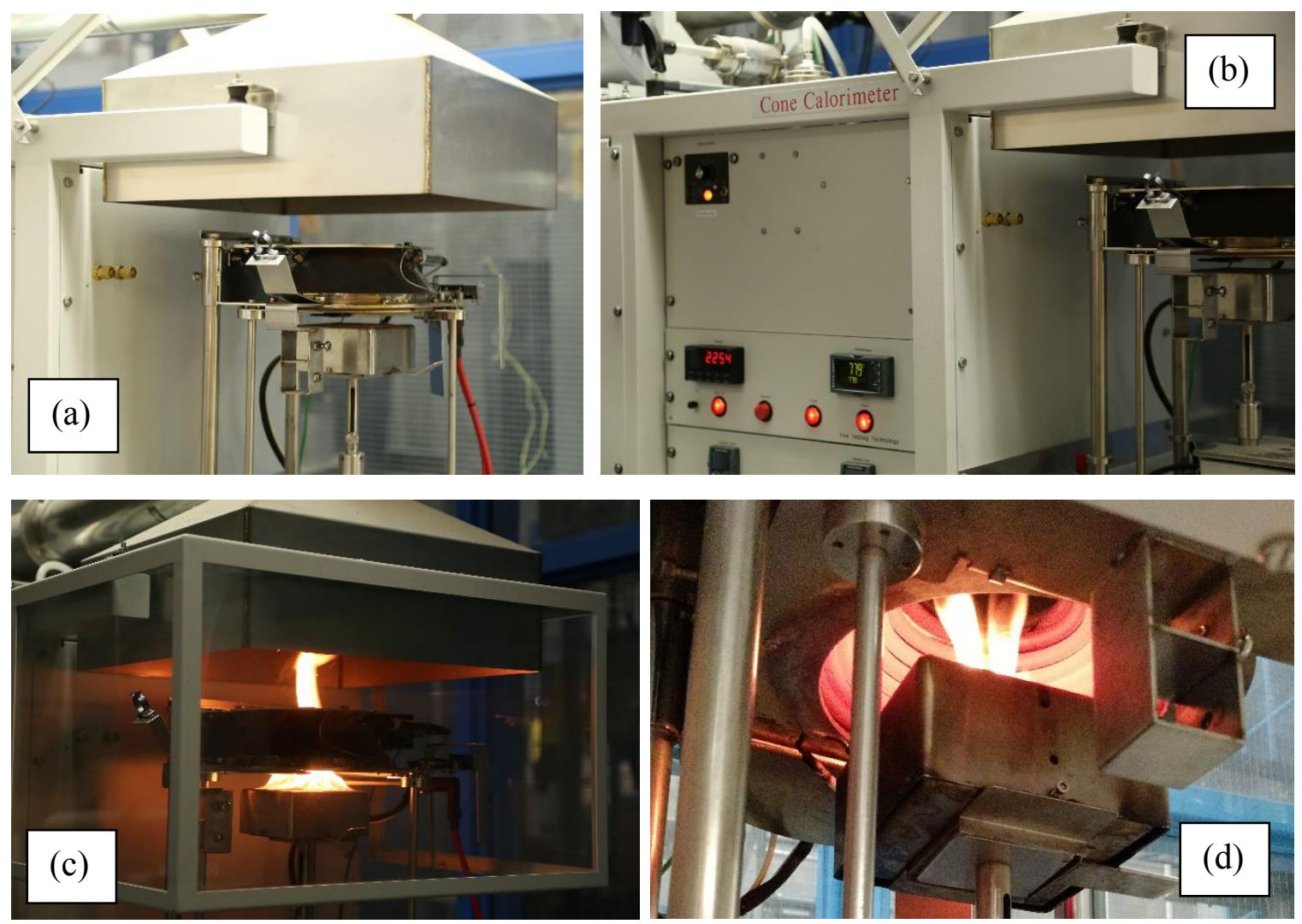

Figure 4.2: Cone Calorimeter apparatus showing (a) the hood used to collect test emissions for calorimetry, (b) the apparatus display, (c) a specimen during heating with the shield deployed and (d) the conical heating element during heating

Several experiments have been done internationally on timber specimens in cone calorimeters. The tests have shown good repeatability, especially at higher heat fluxes (from $25 \mathrm{~kW} / \mathrm{m}^{2}$ to $60 \mathrm{~kW} / \mathrm{m}^{2}$ ) and poorer repeatability at lower heat fluxes such as 10 $\mathrm{kW} / \mathrm{m}^{2}$ (Reszka and Torero 2008). The aforementioned article provides a good description of the heating behaviour of wood exposed to a constant incident radiant heat flux. A few other notable studies which confirm the dependable performance of wood samples in a cone calorimeter include (Spearpoint and Quintiere 2001) and (DiDomizio et al. 2016).

In the current study, samples were wrapped in aluminium foil on all sides except the top surface in order to induce one-dimensional heat flow, and were placed in holders with 
insulation beneath the specimen. The grain orientation of the wood samples was perpendicular to the applied heat flux, and the tests were run with the wood samples in a horizontal configuration. An incident heat flux of $50 \mathrm{~kW} / \mathrm{m}^{2}$ was chosen as a typical exploratory high heat exposure - see commentary in ASTM-E1354 (ASTM 2016b). 30 $\mathrm{kW} / \mathrm{m}^{2}$ was chosen as a comparative lower heat flux exposure, still remaining above the lower heat fluxes reported by Reszka and Torero (2008) to have poor repeatability. All tests were run with unpiloted ignition, as the intent was not to ignite the samples but allow them to sufficiently char with heat exposure. Three, six, ten and fifteen minute exposure durations were considered in order to observe the performance of the remaining adhesive under varying quantifiable char depths. Two samples from the PUR set and one sample from the PRF set were left unheated as control specimens.

This testing procedure also differs from some of the standard tests with regards to the heating, as many standards for evaluating adhesives at elevated temperatures specify a target bondline temperature which must be sustained throughout heating. They are typically done in an oven, where the entire specimen sees uniform heating, and they are tested while still at the elevated temperature. For the purposes of the study, one-sided extreme exposure was used to actually char the wood and leave an unheated wood and adhesive region below the pyrolysis zone. Additionally, tests were done once the specimens had been cooled to allow for detailed observation of the mechanical behaviour and to mimic the previous tests discussed in Section 4.2, which were also done after cooling. This procedure thus offers insights not only into the state of degradation of the adhesive but also into resilience effects. The constant heat flux regime, however, differs from actual fire exposure and the association between incident heat flux, standardized fires, 
and real fires is currently under debate as per the European Cooperation in Science and Technology (COST) Action FP 1404 exploratory initiatives (COST 2014).

\subsubsection{Shear Testing}

In order to create a shear test setup compatible with the sample dimension requirements for the cone calorimeter, a novel apparatus was built, modified from a study on the shear testing of concrete (Smith et al. 2011). This non-standard test method was effective because the testing objective was to perform an exact comparative basis of the remaining shear capacity of several different samples tested under the same setup.

The test apparatus is composed of two identical (inverted) sections which were constructed of steel sections welded together, and adjustable with four pairs of connected bolts on each side, as seen in Figure 4.3 and Figure 4.4. The apparatus was placed between two loading plates on an INSTRON 5582 load actuator through which the wood blocks were placed under a shear force through compression loading. The ASTM D905 standard for timber adhesive shear testing recommends a loading rate of $5 \mathrm{~mm} / \mathrm{min}$ (ASTM 2008). However, due to the smaller area of the shear plane of the samples and the requirement of a longer test duration in order to use the DIC data recording method, the loading rate was set to a slower rate of $1 \mathrm{~mm} / \mathrm{min}$. The shear plane was along the centreline of the wood specimen

that corresponded to the adhesive bond to be tested, shown in Figure 4.4. The load and stroke data was recorded using INSTRON Bluehill software. Slip of the adhesive layer was measured using Digital Image Correlation (described below in Section 4.3.3). 


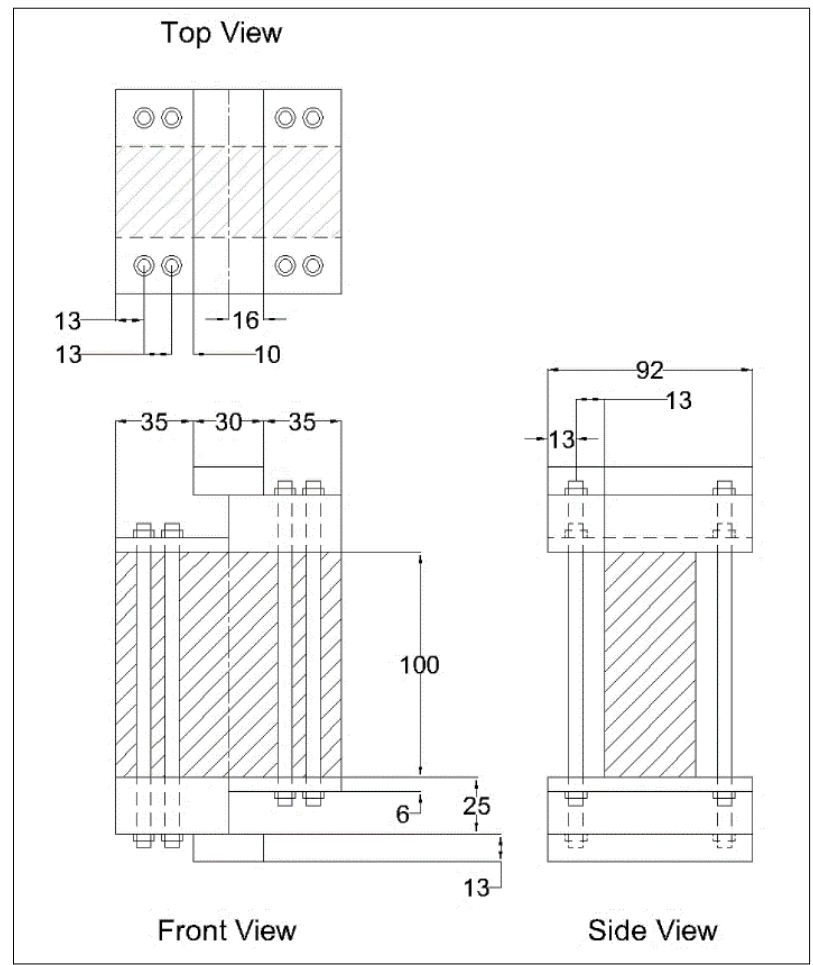

Figure 4.3: Schematic for construction of steel shear test apparatus showing hatched wooden block within apparatus (all dimensions in millimeters)
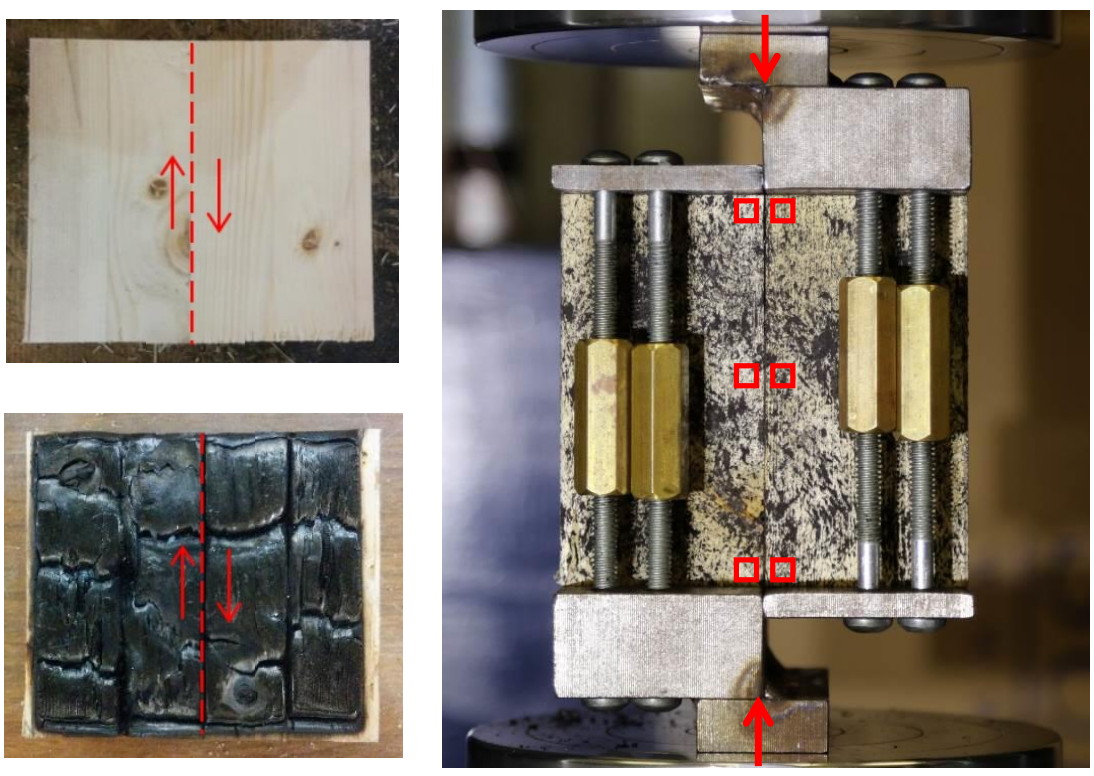

Figure 4.4: Shear plane shown on unheated and heated samples (left) and shear apparatus test setup (right) 
The failure mode here is expected to be due to primarily mode II shearing stresses. Some additional mode I normal stresses on the joint may also arise due to a non-uniform stress profile through the width of the timber on either side of the adhesive centreline. These stress states are expected on Glulam elements in typical service conditions (Pizzo et al. 2003).

\subsubsection{Digital Image Correlation}

Digital Image Correlation (DIC) was used to monitor the deformations of the specimens as they were tested in shear. This is a relatively nascent technique, which was previously shown to be accurate for measuring displacements and strain in polymer and composite specimens and at high temperatures (Gales and Green 2015, Hajiloo et al. 2018). The displacement measurement technique uses a pixel-tracking software, GeoPIV RG, to locate user-specified locations on a series of high-resolution photographs (Stainer 2015). A speckled pattern is painted on the surface to be tracked in order to ensure there is a very unique patch of pixels for the software to track from image to image. The software then records the location of the pixel patch in each photograph captured throughout the experiment, which may be used to compute the displacement and relative deformations of the sample. The accuracy of the program has been quoted as 0.1 pixels (Gales et al. 2012).

In these tests, the slip was measured at 3 points along the shear line: at the top, middle and bottom of the specimens. A sample of the pixel patches used to track the slip are shown in the right-hand image in Figure 4.4. A Canon EOS 5D Mark III DSLR camera was used in conjunction with an automated timer controller at 3 second intervals (the fastest rate at which the camera can record photographs), taking photos of $5760 \times 3840$ resolution. The camera was placed on a professional grade tripod and levelled with both a manual and 
digital level in order to ensure the photos were not distorted in any way and the photo frame remained constant in every photo throughout each test. Gales and Green (2015) gave a complete description of plausible errors in measurement, which included: out of plane movement, lens distortion, levelling and stability (which were dealt with through the rigid tripod and multiple levels); focus of image, resolution and camera vibration from shutter speed (which were dealt with through the camera selection with appropriate photograph intervals and manual focussing); lighting variations (which were dealt with through adequate, constant lighting on the specimens); and self-heating of camera (which was not an issue as mechanical tests were performed after the heating and cooling of the specimens).

\subsection{Experimental Results}

The results of the test series are presented in two sections. First, observations from the heating phase of the experiments are presented along with data on the charring behaviour and depths recorded. Secondly, the mechanical performance of the adhesives is reviewed in terms of strength capacity and load versus slip behaviour. Although the heating phase and resulting char showed highly predictable results, the mechanical results had high variance between the repeated samples. This is expected due to the natural imperfections and non-homogeny of wood and the small size of the samples tested. Nevertheless, emerging trends are seen and discussed from the data analysis presented.

Before testing, the dimensions of each specimen were measured and the mass was recorded using a digital scale. The average density of the specimens was $560 \mathrm{~kg} / \mathrm{m}^{3}$ with the lowest recorded being $517 \mathrm{~kg} / \mathrm{m}^{3}$ and the highest $600 \mathrm{~kg} / \mathrm{m}^{3}$. This average density corresponds exactly with the manufacturer specified density (Nordic Wood Products 2015). The steady 
state moisture content was read on the lab-acclimatized specimens using a two-pronged moisture meter. The average moisture contents recorded for the samples was around $6-7 \%$.

\subsubsection{Heating Observations and Char Results}

The heating behaviour of the samples were very predictable. For the $30 \mathrm{~kW} / \mathrm{m}^{2}$ incident heat flux exposure, flaming ignition was not seen in the majority of the samples. Rather, smouldering was observed without flaming with the exception of two PUR samples heated for a 15 minute duration which began flaming after around 7-8 minutes of exposure. For the $50 \mathrm{~kW} / \mathrm{m}^{2}$ incident heat flux exposure, flaming ignition in all samples was seen after around 30-40 seconds of exposure. There was little difference between the heat release rates (HRR) of the samples with different adhesives, as is seen in the sample HRR curves shown in Figure 4.5.

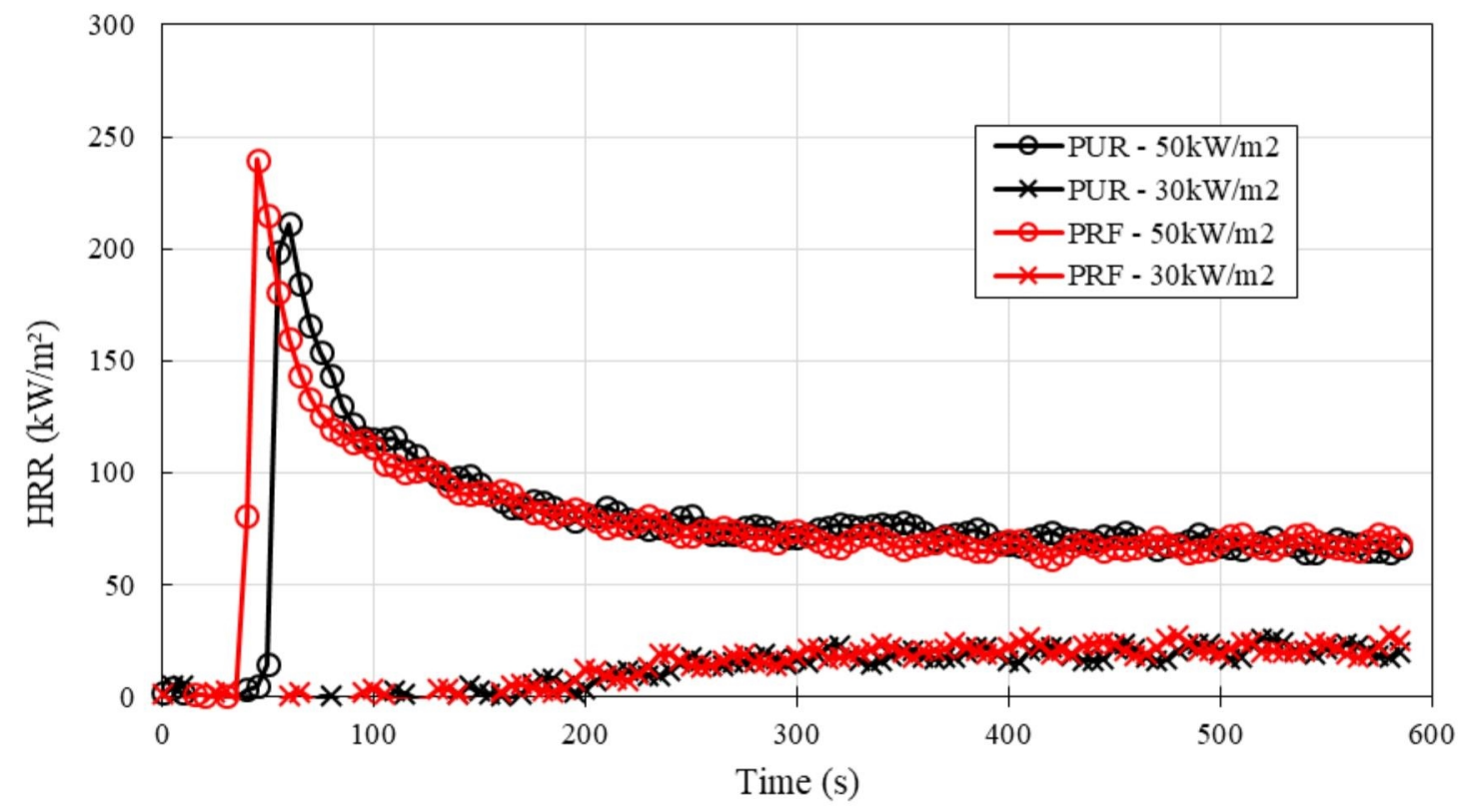

Figure 4.5: Sample heat release rate curves of wood samples measured by cone calorimeter; one sample of each adhesive type for both $30 \mathrm{~kW} / \mathrm{m}^{2}$ and $50 \mathrm{~kW} / \mathrm{m}^{2}$ incident heat flux exposures for 10 minute duration tests are shown 
From Figure 4.5 it is observed that the peak heat release was reached in the $50 \mathrm{~kW} / \mathrm{m}^{2}$ samples just after flaming ignition between $200-250 \mathrm{~kW} / \mathrm{m}^{2}$, and then decreased as char built up and the burning of the fresh wood slowed to a plateau around $70 \mathrm{~kW} / \mathrm{m}^{2}$. In the samples exposed to $30 \mathrm{~kW} / \mathrm{m}^{2}$ the HRR slowly increased to a plateau and maximum rate of about $20-25 \mathrm{~kW} / \mathrm{m}^{2}$.

After the samples were broken in half by shear testing, the average char depth at the centreline was measured. The char depth was taken as the average of three points along the centreline (which were typically within $1 \mathrm{~mm}$ of each other). The char depths of each sample versus their heating durations is plotted in Figure 4.6, where it is observed that the char depths for the higher heat exposure were typically higher than those of the lower heat exposure. A clear correlation between heating duration and char depth can be seen.

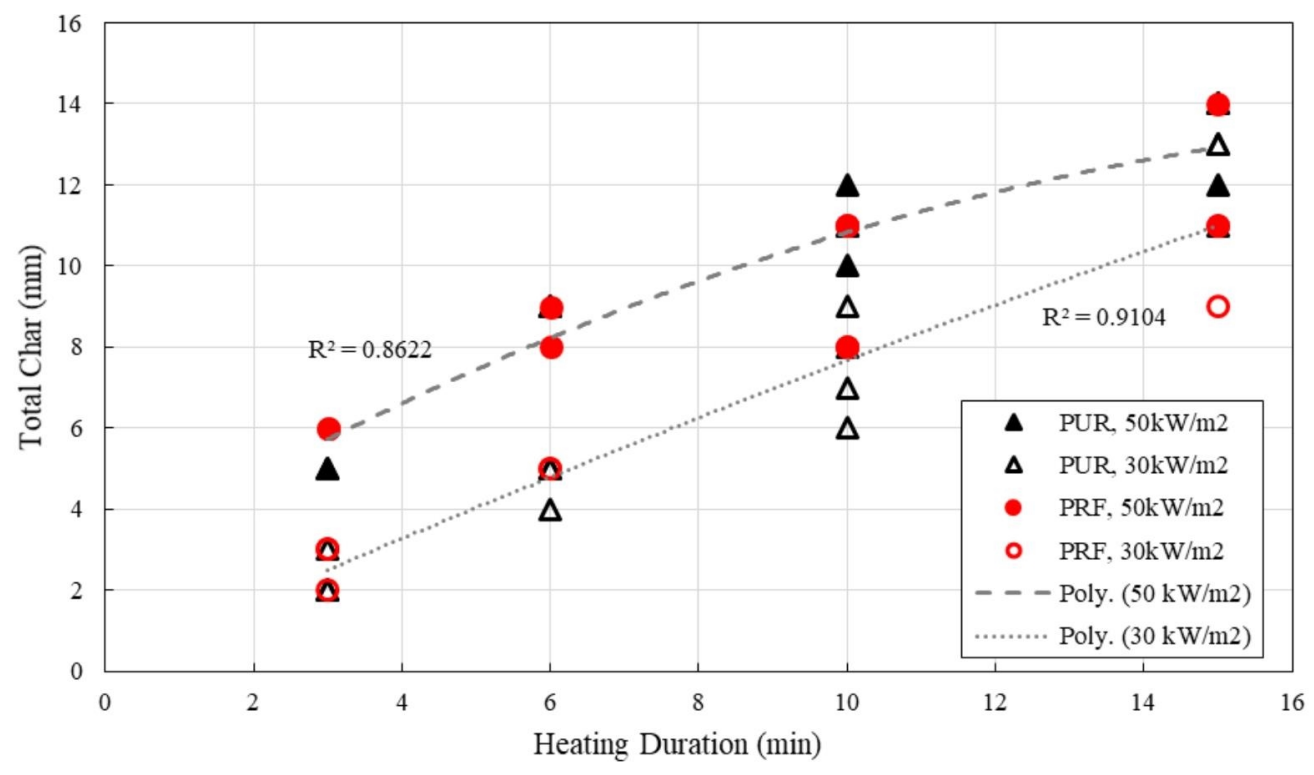

Figure 4.6: Total char depths measured on samples versus the heating duration The data sets were best represented by second-order polynomial functions which are depicted on Figure 4.6 with moderate correlations (an R-squared value of around 0.9). The 
trend for both the $50 \mathrm{~kW} / \mathrm{m}^{2}$ and $30 \mathrm{~kW} / \mathrm{m}^{2}$ exposures decreased in slope with longer heating durations, while this is more pronounced in the higher heat exposure. This indicates that the charring rates slow over time which is a well-known phenomenon, as the char layer builds up and inhibits heat transfer. This is observed even more clearly in Figure 4.7 where the charring rates (averaged over the entire heating duration) are plotted against the corresponding heating duration.

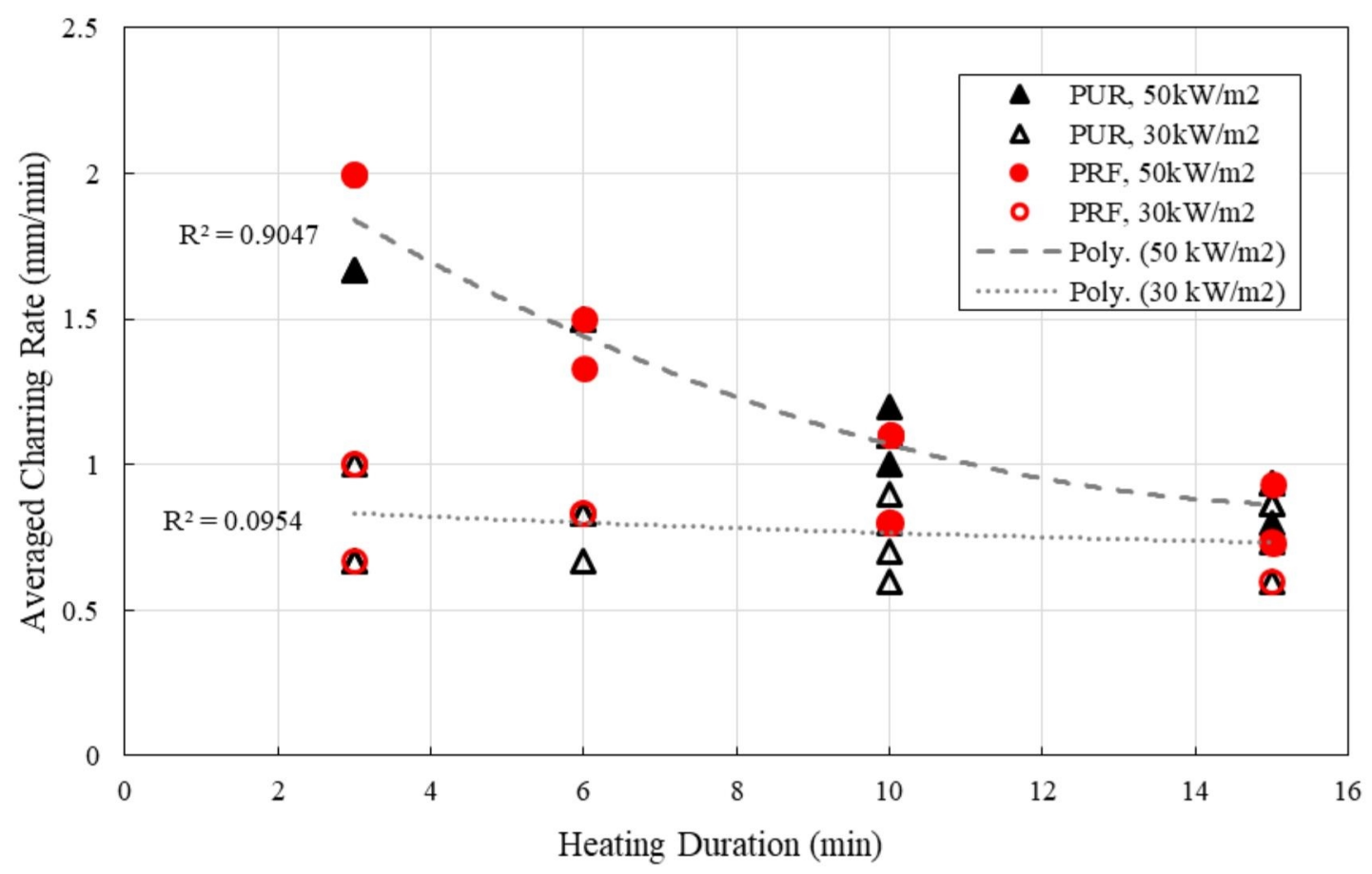

Figure 4.7: Charring rate averaged over the entire heating duration for each sample versus its heating duration

A moderate correlation is seen with the higher heat exposure samples with a second order polynomial function with an R-squared of 0.9 . The trend clearly shows a decreasing rate of charring with heating duration which approaches a minimum near to that of the lower heat flux samples. The lower heat exposure samples had much more variance which could be due to the fact that some samples flamed and some only smouldered. A fairly constant 
charring rate is seen there which indicates that not enough of a char layer built up to slow the rate of charring. The higher heat flux charring rate trend tends to approach the lower heat flux around a minimum value of $0.75 \mathrm{~mm} / \mathrm{min}$ which indicates that this might be a minimum rate of charring that may be seen in timber above the pyrolysis temperature. This corresponds well with the code recommended charring value of $0.7 \mathrm{~mm} / \mathrm{min}$ for standard fire exposures. Overall, the charring behaviour of the samples was very uniform and repeatable. Two sample images of charred specimens are shown in Figure 4.8.
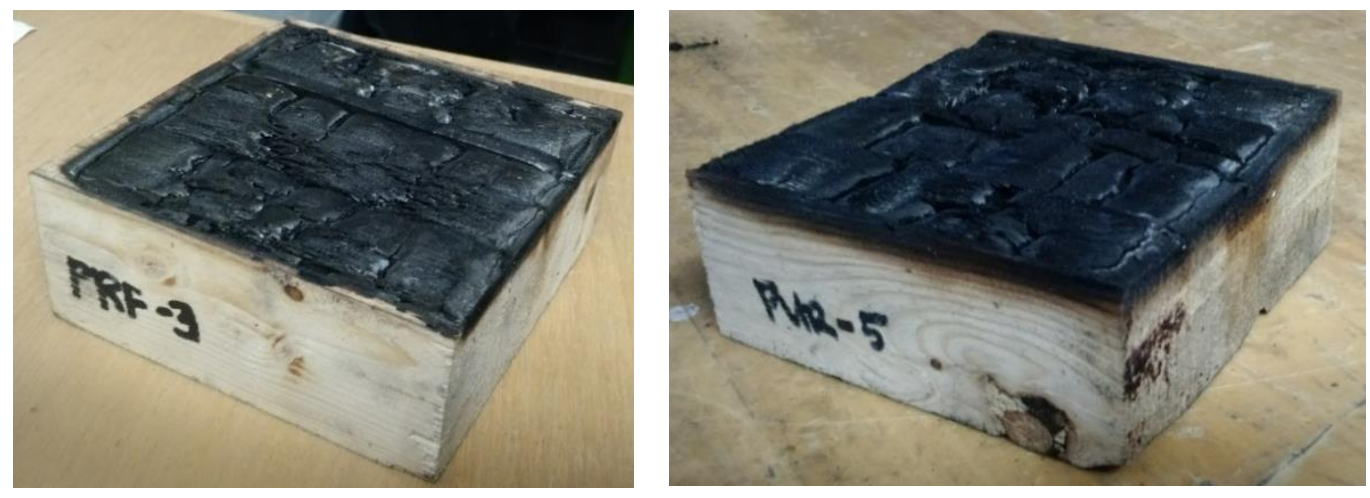

Figure 4.8: Samples after burning exposed at $50 \mathrm{~kW} / \mathrm{m}^{2}$; on the left a PRF sample exposed for 3 minutes and on the right a PUR sample exposed for 6 minutes

\subsubsection{Adhesive Strength Capacity}

As was demonstrated in Section 4.4.1, the charring of the samples in the cone calorimeter was very repeatable. Additionally, the char depths seen were close to uniform along the surface of the specimens. The area beneath the char layer was used to calculate the failure stress of each sample at the peak load reached in the test before failure. If the adhesive within the wood region below the char front of the samples was also unharmed, the failure stress of each sample should be equal (although the peak load would be lower due to loss of cross-sectional area). Figure 4.9 and Figure 4.10 show the trends in the failure stresses of all samples for the PUR and PRF adhesives, respectively. 


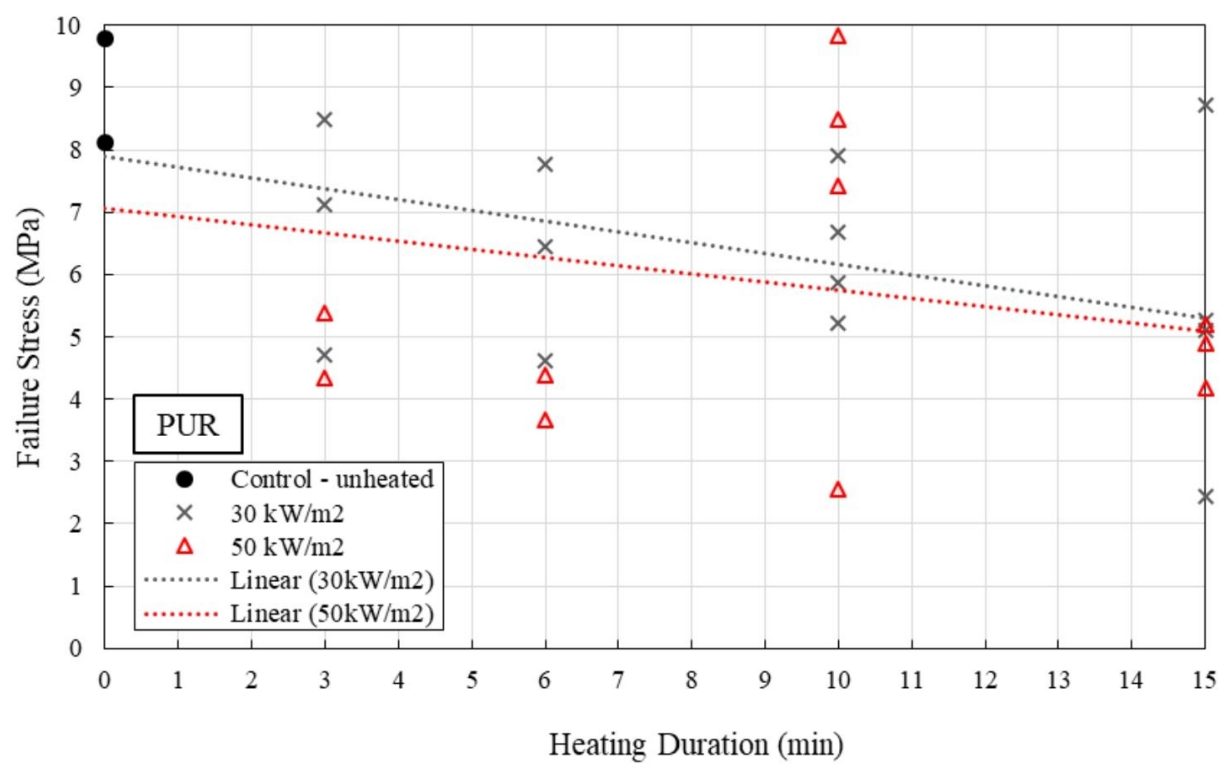

Figure 4.9: Failure stress of each PUR sample tested compared to heating duration of that sample with linear trend lines depicted for each heating exposure

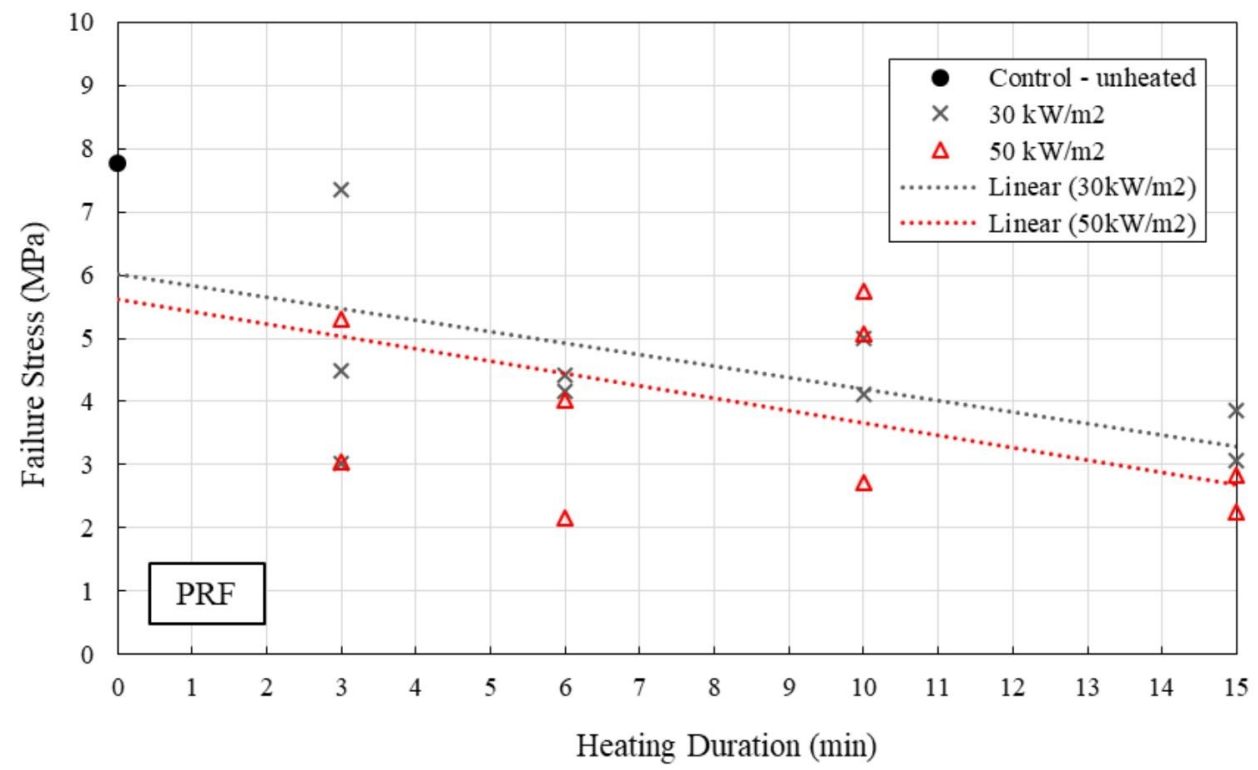

Figure 4.10: Failure stress of each PRF sample tested compared to heating duration of that sample with linear trend lines depicted for each heating exposure

As can be seen in Figure 4.9 and Figure 4.10, there was a large amount of variance in the failure stresses of the samples. However, the linear trend lines depict a downwards trend 
of the stresses, decreasing in strength with heating duration, although the $\mathrm{R}^{2}$ values of these trends are very low (less than 0.4 in all cases). This indicates that the adhesive beyond the char layer is affected by the heat and has lost some additional strength unaccounted for in the sacrificial char method, which is further investigated below. Nevertheless, the excessive variance in the sample performance represented in the low correlations of the trends warrants further testing of even more samples.

A correlation is made between the remaining shear capacity of the samples and their respective remaining shear area. In theory, if the adhesive held $100 \%$ of its strength after all heat exposures, these two values would correlate exactly. That is, if a sample had $80 \%$ of its shear area left after heating, the remaining shear capacity should be $80 \%$. This relationship is shown as the red dashed line in Figure 4.11. Any sample falling below this line represents a sample that did not retain its full adhesive strength - i.e. the loss of shear capacity was greater than the loss of area.

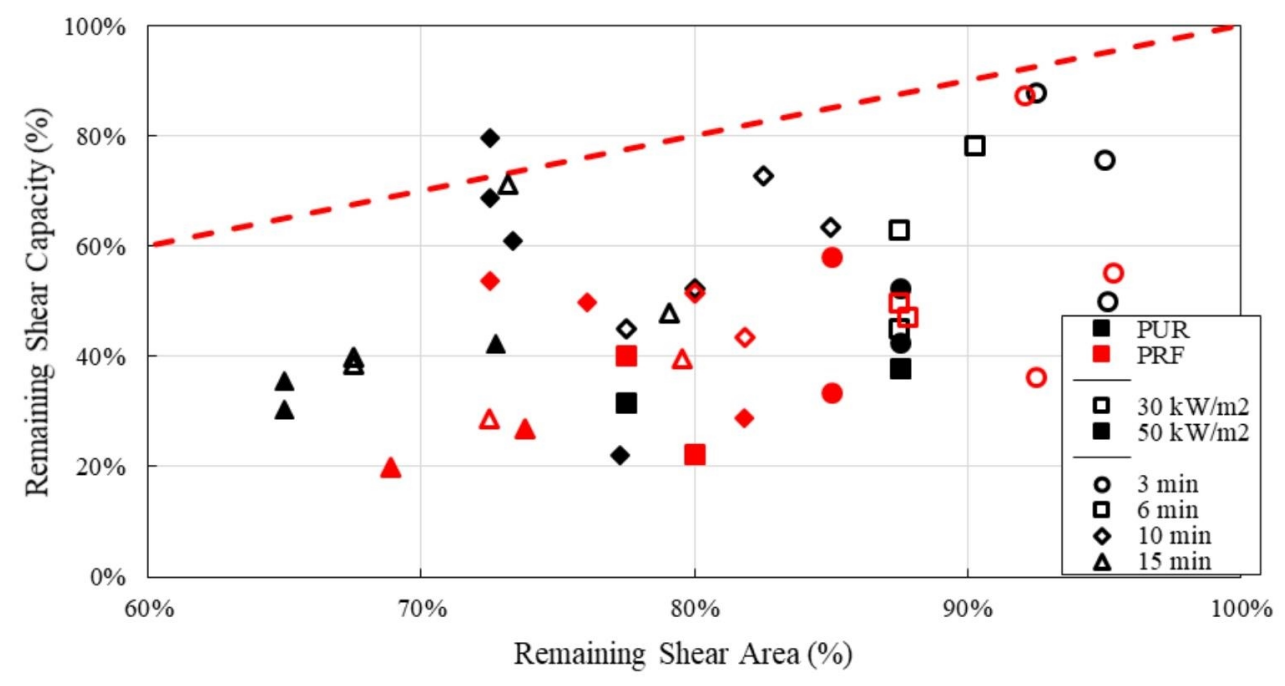

Figure 4.11: Comparison of remaining shear capacity versus area for all samples 
From Figure 4.11 it can be seen that the majority of the samples fell well under the perfect correlation line, with most samples having a remaining area beneath the char of above $60 \%$, but many samples falling to as low as $30 \%$ remaining capacity. On average, the samples had a remaining capacity $32 \%$ below their remaining shear area. Further, the capacity of the PRF samples fell an average of $40 \%$ below their remaining area while the PUR samples were $26 \%$ below. This could indicate a difference in the adhesives or in the way they were prepared, with the PUR bonds being produced by the manufacturer while the PRF bonds were produced in the lab. Additionally, from Figure 4.11 there is no clear trend or distinction developed within any of the heating durations or exposures.

Thus, although there is ample scatter within the data seen in Figure 4.9 and Figure 4.10, it is clear from Figure 4.11 that the adhesive performance falls below that of which it is expected.

A comparison was made with the remaining capacity that the code recommended sacrificial charring method would have predicted. A nominal charring rate of $0.7 \mathrm{~mm} / \mathrm{min}$ was used plus a zero-strength layer of $7 \mathrm{~mm}$. It should be noted, however, that these recommended charring rates are not validated for exposures other than a standard-fire, and are thus not totally applicable to a constant heat flux radiant exposure, but are included here for comparison. As can be seen in Figure 4.12, when compared with the actual remaining shear capacity, the code predictions still fall significantly below the perfect correlation line. This new data set is plotted in grey in Figure 4.12 compared to the original data set in black.

There may be two possible causes for the reduced strength of the samples. The first is that, due to the small thickness of the samples, all of the adhesive beyond the char layer may be reduced in strength by a certain percentage corresponding to the lower capacities 
mentioned above. However, since the adhesives must have been tested to the standards discussed in Section 2.1.4, the adhesive should retain full strength for temperatures below the charring temperature of wood. Secondly, the adhesive may be full strength at a certain depth beyond the char layer, but an additional layer of adhesive beyond the encroached char front may have been degraded. In other words, a larger zero-strength layer may be present than is recommended in codes. This zero-strength layer has also been challenged before, for example as discussed in Section 2.1.3 from Lange et al. (2015). It is likely that the adhesive and therefore the combined strength of the engineered wood product may have degraded to a certain depth beyond the char front, after which the remaining adhesive there may be assumed to have full strength. This effect is investigated further below. Another factor that may contribute is the loss of strength of the wood above $100^{\circ} \mathrm{C}$, as many of the failures were a combination of wood and adhesive failure, so the total reduction in strength cannot be attributed solely to the adhesive degradation. The suggestions herein are thus highly conservative. 


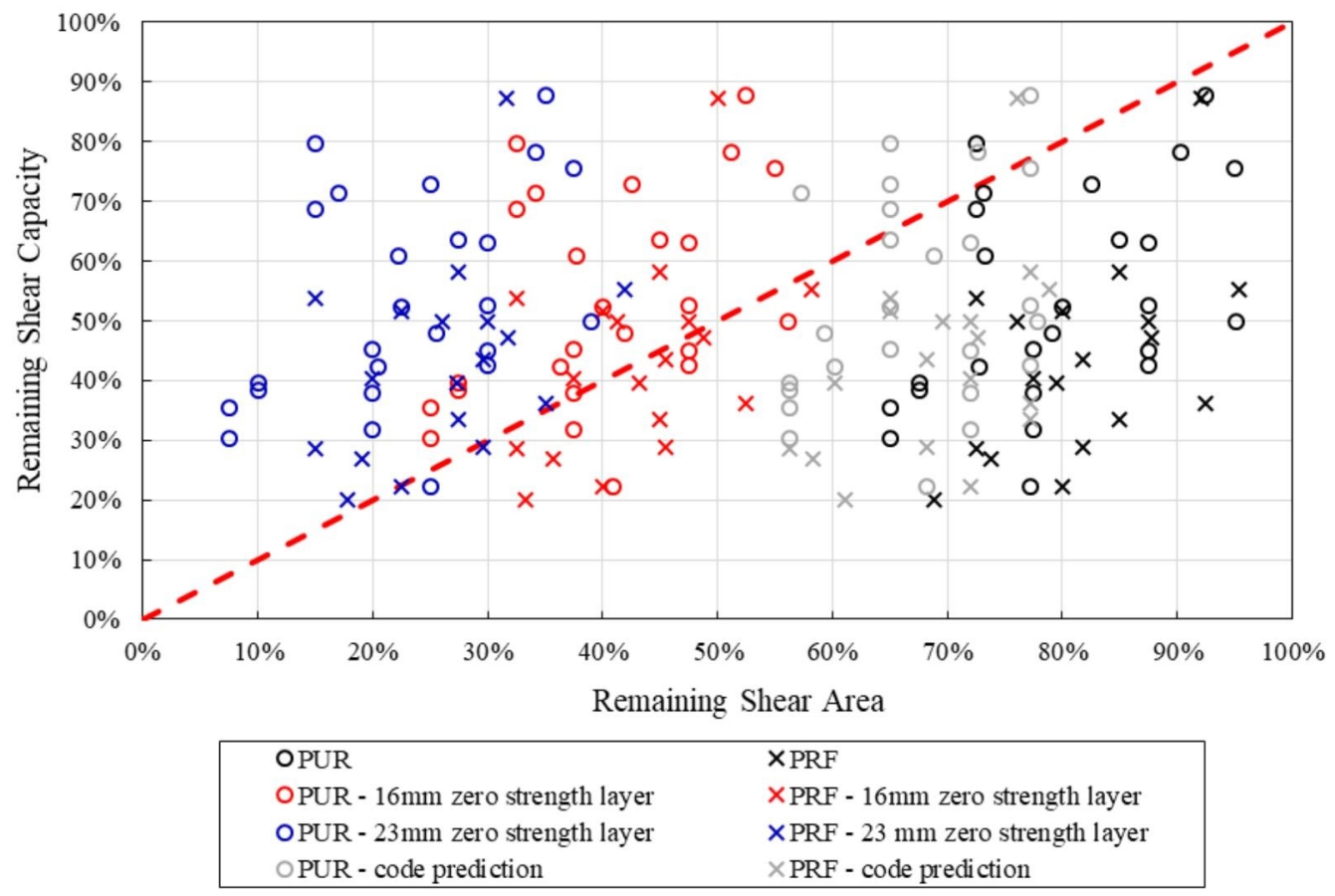

Figure 4.12: Remaining shear capacity of samples versus different predictions of actual remaining shear area at full adhesive strength, compared with the measured char depths and the code predicted sacrificial char method

If the adhesive is assumed to have full strength at a certain depth beneath the char layer, than the area of that full-strength zone may be computed using the peak shear capacity of each sample. The full strength was taken as the average failure stress of the control specimen for each adhesive, calculated by dividing the failure load by the corresponding shear area. It was assumed that this full-strength zone spans the full height of the samples, and that the depth is measured through the thickness of the sample from the unheated face, a schematic of which is shown in Figure 4.13. In this way, the penetration of the zerostrength zone may be calculated using the recorded failure load of each sample and the measured char depth by Equation 4.1 where $d_{\text {zero }}$ is the calculated zero strength layer depth, $d_{c h a r}$ is the measured char layer depth of that specimen, $t_{0}$ and $h_{0}$ are the original 
thickness and height (respectively) of that specimen, $\sigma_{\text {full }}$ is the calculated full strength of the adhesive and $P_{\text {failure }}$ is the failure load of that specimen:

$$
d_{\text {zero }}=t_{0}-d_{\text {char }}-\left(\frac{P_{\text {failure }}}{\sigma_{\text {full }} \cdot h_{0}}\right)
$$

Equation 4.1

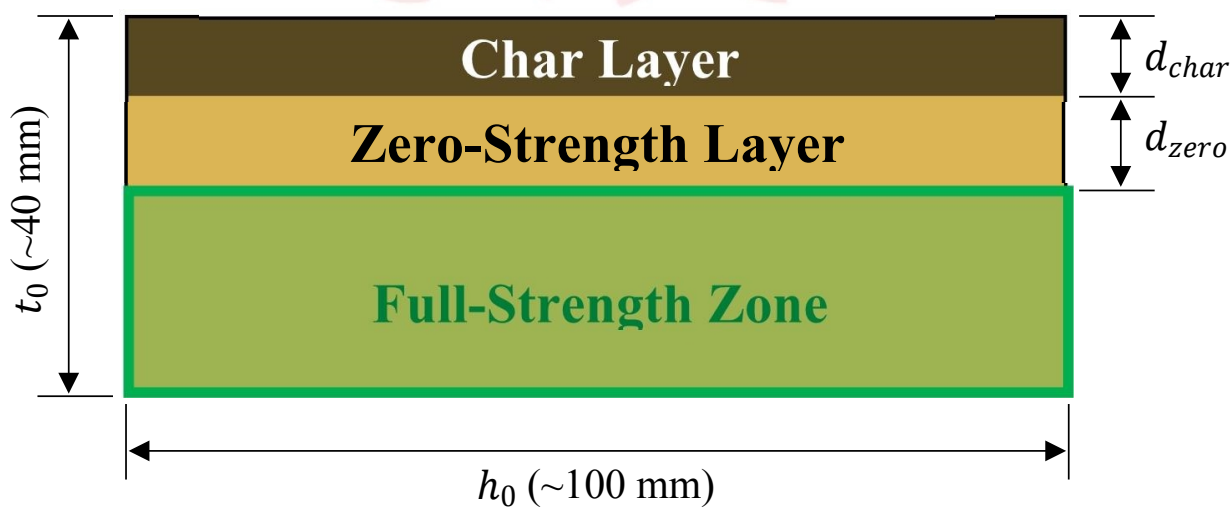

Figure 4.13: Schematic of cross-section through shear plane in specimen showing the area used to calculate the full-strength zone and thus the additional zero-strength layer The results from all calculations on the sample data may be found in Appendix D. On average, the zero-strength layer penetrated a depth of $13.3 \mathrm{~mm}$ beyond the base of the char zone. Separated for each adhesive, this value was an average of $16.2 \mathrm{~mm}$ for PRF samples and $10.7 \mathrm{~mm}$ for PUR samples. For comparison with the original shear ratio predictions, $16 \mathrm{~mm}$ was added to the measured char depth of each sample and a new remaining shear area was calculated. This new data set may also be compared to the original and codepredicted data sets in red in Figure 4.12. It can be seen that increasing the zero-strength zone to $16 \mathrm{~mm}$ below the measured char depth of the samples results in a much better 
prediction of the remaining strength, with the majority of the samples falling over the line. A more conservative value of $23 \mathrm{~mm}$ below the char depth could be used to give a $95^{\text {th }}$ percentile of samples falling above the line, which is shown as the blue series in Figure 4.12.

\subsubsection{Adhesive Slip Behaviour}

In addition to the strength performance of the specimens, digital image correlation was used to record the displacements during loading and the slip behaviour of the adhesive lines was investigated. However, DIC was not able to be completed for all specimens as several of the test image sets came out with a large fraction of corrupted photos after the upload to the computer from the camera. Of the samples with complete sets of images, some interesting observations may be made. The slip was calculated using the procedures outlined in Section 4.3.3 and was averaged over the three measurement locations to represent the average slip of the adhesive line. A comparison is made between the loadslip behaviour of several representative samples under $50 \mathrm{~kW} / \mathrm{m}^{2}$ incident heat exposure with PUR and PRF adhesives in Figure 4.14 and Figure 4.15, respectively. It is clearly observed in both figures that as the heating duration increased, lower loads were reached for the same level of slip. This indicates that the adhesive stiffness or rigidity has degraded

over increased periods of heating. The PRF samples also seemed to experience less slip than the PUR samples. 


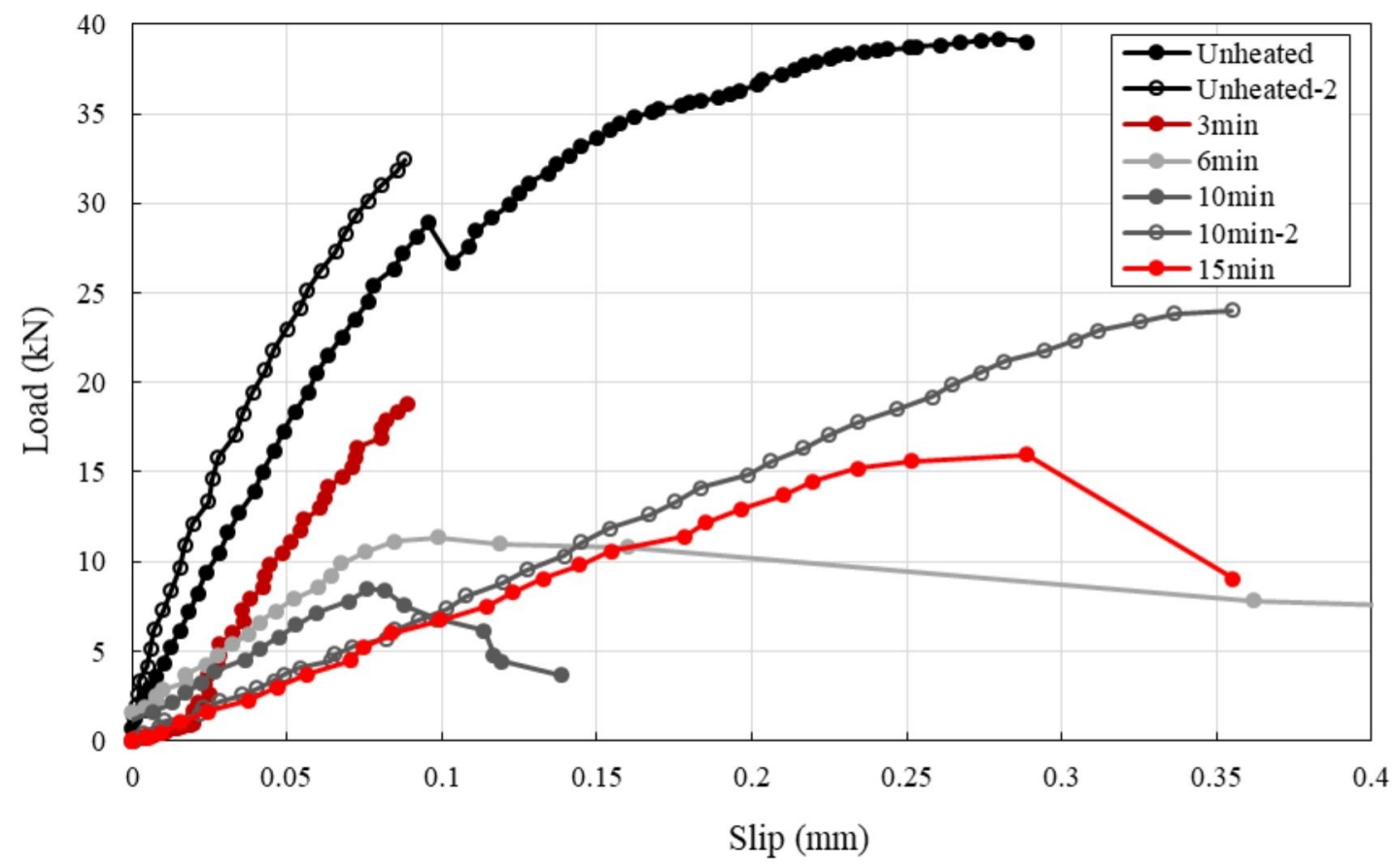

Figure 4.14: Load versus slip behaviour for several samples heated for different durations under a $50 \mathrm{~kW} / \mathrm{m}^{2}$ incident heat flux, with PUR adhesive

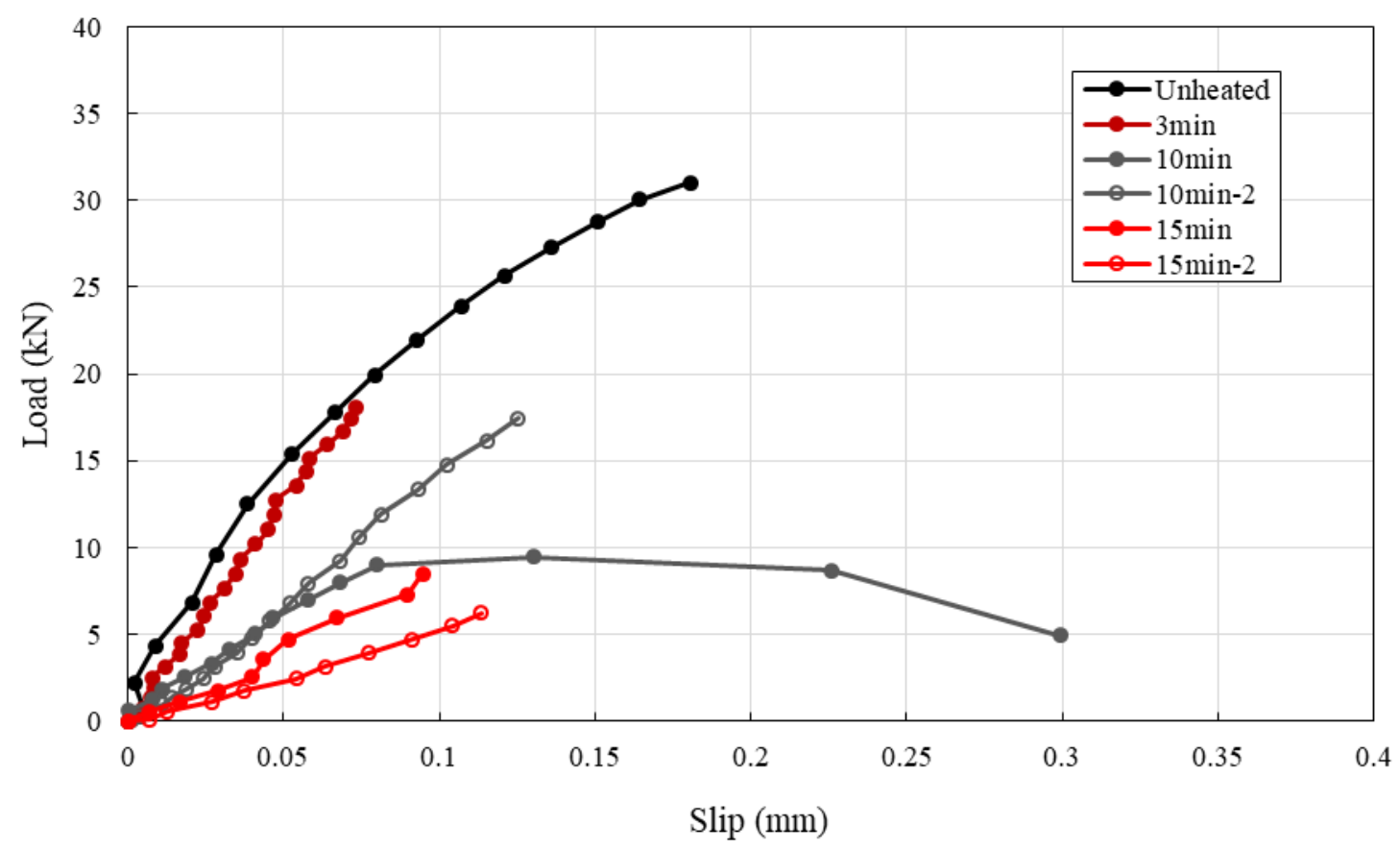

Figure 4.15: Load versus slip behaviour for several samples heated for different durations under a $50 \mathrm{~kW} / \mathrm{m}^{2}$ incident heat flux, with PRF adhesive 
Finally, Figure 4.16 shows the load versus slip at failure for every specimen in which DIC could be completed. Linear trend lines were plotted in order to gain insight on any emerging trends, although the data still presented ample scatter and the correlations were not strong. However, clear trends can be seen that have similar slopes of increasing slip with load, but the longer the heating duration, the lower the loads corresponding to the same slip. This also corroborates the trends seen in Figure 4.14 and Figure 4.15.

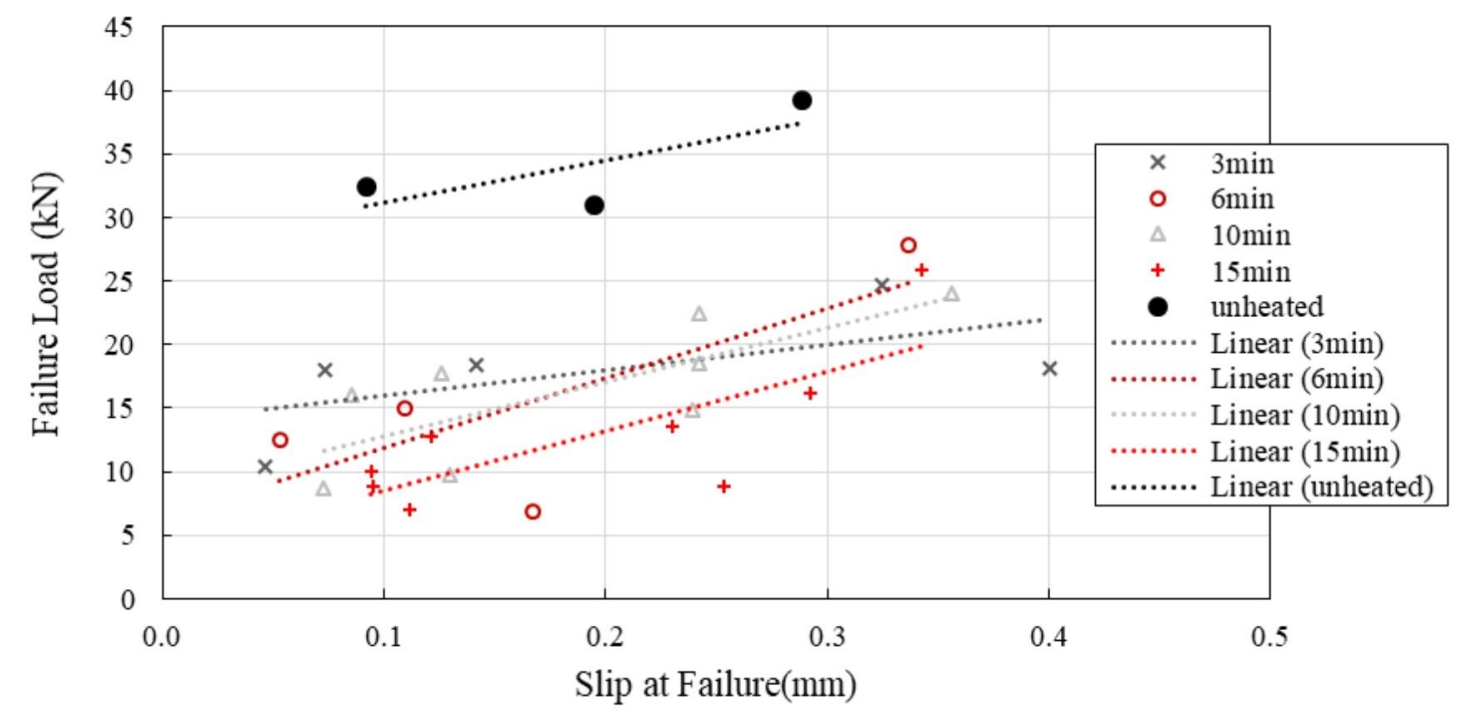

Figure 4.16: Slip at failure versus failure load for each specimen for which DIC was able to be completed, with linear trend lines included for each heating duration

\subsection{Conclusions and Recommendations}

The heat release rates of the timber were as expected and the charring behaviour was very predictable and uniform. The charring rates showed moderately strong trends decreasing with heating time for the $50 \mathrm{~kW} / \mathrm{m}^{2}$ incident heat flux tests due to char build up. These rates approached the constant charring rates of the $30 \mathrm{~kW} / \mathrm{m}^{2}$ incident heat flux tests which 
indicates that this may be a minimum charring rate at which the softwood Glulam samples would char once a char front has developed, around $0.75 \mathrm{~mm} / \mathrm{min}$.

Although the high variance in the results typical of wood was seen, some clear trends emerged in the strength and slip properties of the adhesive with varying heat exposures. A clear downwards trend was seen in the strength of all samples when plotted against heating duration, both for the $50 \mathrm{~kW} / \mathrm{m}^{2}$ and the $30 \mathrm{~kW} / \mathrm{m}^{2}$ heating regimes. By only considering the charred layer or the sacrificial char layer from building code recommendations, the remaining full-strength zone and thus the predicted remaining capacity of all specimens was greatly over-estimated. An average of $10.7 \mathrm{~mm}$ for PUR and $16.2 \mathrm{~mm}$ for PRF should be added to the zero-strength zone below the visible char layer in order to accurately predict the remaining capacity of the samples. For a conservative $95^{\text {th }}$ percentile value for all samples tested, the zero strength layer could be increased to $23 \mathrm{~mm}$. The samples tested were very small scale, so larger scale members should be tested. A greater area of unheated wood behind the char layer would likely increase the remaining capacity of the wood so such a drastic addition to the zero-strength layer may not be required. Additionally, this value may not be applicable to different fire exposures, including the standard fire, or for other adhesive types or products of the same type from different manufacturers. Furthermore, the issue of highly conservative measures such as those presented here stems from the underlying problem of not truly understanding the material behaviour. Simplified numbers are desired for use in codes and guidelines, but such oversimplifications (as opposed to having a detailed knowledge of the material) result in exceedingly conservative quantifiers. Additionally, effects introduced by cracks propagating into the adhesive layer (formed from wood defects, finger joints or timber failure, etc.) introduce stress 
concentrations and have been found in the past to be a large influence on adhesive failure, so this factor would also affect results in larger specimens. Perhaps a more feasible solution is to recommend that a new standardized test be developed for timber adhesives which quantifies the performance beyond the char layer in burnt engineered timber so that individual adhesives may be evaluated.

The slip behaviour also exhibited trends of increased slip with load on the samples for which DIC was completed. This indicates that the heated adhesive loses some stiffness compared to the unheated samples. It is important to note that the samples were tested after having been cooled to correspond with the previous testing discussed in Section 4.2, and thus may be applicable for the post-fire investigation of fire-damaged Glulam elements, but may not necessarily be applicable for in-fire conditions.

New adhesives which do not degrade as much in fire would be desirable. One such adhesive has been recently under testing by NIST which is a silicone-based adhesive, showing promising properties for fire resistance. Various other adhesives have been receiving attention and improvement for fire resistance including Melamine-Formaldehyde (MF) and Melamine-Urea-Formaldehyde (MUF) adhesives. Adhesive degradation and delamination may pose a challenge in structural capacity and fire load contributions in buildings with exposed timber, so more tests as indicated above should be done in order to quantify the effects in full-scale structures. 


\section{Chapter 5: Conclusions and Recommendations}

\subsection{Summary of Findings}

There has been ample research and interest in novel timber products and structural schemes over the past decades. An increased environmental conscientiousness in society and the abundance of wood in Canada has inevitably lead to the desire for more timber construction, and the proposed NBCC 2020 amendments to allow for up to 12-storey timber structures. In order to increase the opportunity for timber products to be used in large-scale commercial construction, novel building systems such as PT timber are required. Such complex building systems likely require detailed design solutions for approval. In order to propose design tools which benefit practitioners for such novel systems and materials, it is of the utmost importance to have a deep understanding of the material's performance.

The ultimate goal of this research study was to facilitate guidance for the use of PT timber as a structural system in Canada. With this background and the experimental program for this research, along with the numerical model development, the results will offer insight into the detailed behaviour and fundamental properties of innovative timber materials and systems. A model was developed in Abaqus in order to investigate the feasibility of available FEM software packages to model PT timber in fire conditions, and to eventually inform a tool for practitioners to design and implement the structural system. Additionally, the performance of timber adhesives after fire damage was examined after previous research suggested further investigation was required. The following sections detail the findings of each of these two research components. 


\subsubsection{Finite Element Modelling of Post-Tensioned Timber Beams}

A numerical model scheme was developed in Abaqus using finite element method (FEM) modelling techniques for post-tensioned (PT) timber beams under both ambient and fire conditions, with detailed attention to material model comparison and selection. The following conclusions were drawn:

- The Engineering Constants elasticity definition combined with the Hill's Potential Function for Plasticity are currently the best available material models within Abaqus (and in all the reviewed available FEM software packages) to demonstrate the behaviour of post-tensioned timber in ambient and fire conditions.

- The model developed may be used with manufacturer specified material parameters along with Eurocode recommended thermal properties and reduction factors when used in conjunction with standard fire testing. Thus, the material parameters were not calibrated to achieve the specific results of the past experiments.

- The model was validated against four ambient and three furnace beam tests which were simply supported in a four-point loading scheme, with reasonable results for ambient tests and very accurate results for fire exposed tests.

- The model failure loads for three of the four ambient beams correlated quite well to the experimental results, within $10 \%$, two of which were over-predicted and one of which was under-predicted. In the fourth beam, the model severely overpredicted the failure load of the beam by about $50 \%$. Additionally, the failure mechanisms and exact load-deflection behaviour were not observed to correlate with the experimental data, likely due to the lack of asymmetrical constitutive properties and crack initiation within the model. 
- The heat transfer models were able to predict the thermal gradients which were available for comparison in the beam furnace tests quite well using the Eurocode parameters for thermal properties and the standard fire exposure through radiation and convection. The cavity approximation provided improved results for thermal gradients when compared to adiabatic cavity surfaces. The assumption that the tendon heat transfer and thermal expansion was negligible was okay in this study.

- The char depths were able to be predicted within $5 \mathrm{~mm}$, and were under-predicted as the model did not account for any additional charring in the cooling/ extinction phases. This difference is large compared to the experimental data for the shorter duration test (25\%) as the char depth is small and typically faster in the beginning of the fire exposure, which resulted in this gross under-prediction. However, in the longer fire durations, the char depth was predicted to as low as $2.5 \%$ error.

- The load-deflection behaviour for the thermo-mechanical simulations was predicted quite well, with the model beam behaviour being slightly less flexible towards the end of the analyses. This was likely due to the lack of crack development within the model, which would have caused the experimental beams to lose stiffness and fail more gradually. However, the model was able to quite accurately demonstrate the failure times for the beams, to within two minutes of the experimental failure times. Additionally, the failure mechanisms observed in the numerical models correlated quite well with the observable failures in the furnace tests.

In general, the developed numerical model showed highly promising results for demonstrating the loading and failure behaviour of PT timber beams. Abaqus is a great 
research tool to establish the capabilities and shortcomings of current FEM technology, which may then be very useful in establishing a practitioner-accessible design tool.

\subsubsection{Timber Adhesive Performance after Fire Damage}

The performance of structural adhesives in timber has been established as a major research need for the fire safety of timber structures. The experimental results found herein may aid in the establishment of more standard testing or a change in the design methods for engineered timber products. The following conclusions were drawn:

- The S-P-F Glulam specimens showed very constant charring behaviour under the two radiant heat exposures in the cone calorimeter apparatus. The high heat exposure $\left(50 \mathrm{~kW} / \mathrm{m}^{2}\right)$ samples saw an initially high charring rate which decreased to a plateau around the same constant charring rate which was seen in the low heat exposure $\left(30 \mathrm{~kW} / \mathrm{m}^{2}\right)$ samples, around $0.75 \mathrm{~mm} / \mathrm{min}$. The exposure durations were short, up to only 15 minutes, and the samples were small.

- The mechanical results were highly variable due to the natural variance and defects in wood products, amplified at such small sample sizes. However, clear downward trends in the strength of the remaining uncharred timber-adhesive bonds were observed.

- Based on the experimental results, additional zero-strength layer thicknesses were estimated beyond the char front to account for the loss of strength. The average additional layer in PRF samples was $16.2 \mathrm{~mm}$ and in PUR samples was $10.7 \mathrm{~mm}$ (compared to the code suggestion of $7 \mathrm{~mm}$ ). A $95^{\text {th }}$ percentile value for samples having conservatively predicted remaining strengths, an additional zero-strength layer of $23 \mathrm{~mm}$ was calculated. 
Overall, the results quite significantly suggested that the adhesive may degrade beyond the char layer. These suggested zero-strength layer values are highly conservative because of the very small dimensions of the sample. Additionally, some of the samples failures were partially in the wood, so some of the reduction in strength is not only from adhesive degradation but also due to any heating of the timber beyond the char front. Furthermore, this layer may not be applicable to different fire exposures, including the standard fire, or for other adhesive types or products of the same type from different manufacturers.

\subsection{Recommendations and Future Research}

Broadly, there is ample research to be done on novel timber products and their response to fire. As was stated in Section 1.3, many topics were outside the scope of this research including ignition, flame spread, smouldering, charring models, non-standard fire exposures, delamination, cooling phases, and self-extinguishment. Specific to this study, some recommendations are made for the continued improvement of knowledge of engineered and PT timber for both researchers and practitioners.

\subsubsection{Academic Recommendations}

In terms of the numerical model, the next step to improving the results (especially for the ambient models which had less accurate comparisons) would be to create a material model which can capture the current shortcomings, including the definition of an asymmetrical constitutive model in tension and compression, and the initiation and development of cracking with propagation along the grain of the wood beams. These would allow for more accurate simulations of stresses arising within the beams and loss of stiffness due to cracking. 
The model should also be tested in an a priori format with large-scale four-point loading PT timber beam tests in order to truly evaluate the ability of the model to predict the thermal and structural behaviour. As mentioned in the previous section, it will also be beneficial to develop a design tool accessible to practitioners for the implementation of safe and efficient PT timber structures, whether it be a step-by-step procedure to create a model of the type developed herein or a special purpose software for these types of analyses.

As far as the adhesive testing goes, it will be important to test larger specimens in order to get more accurate results. This may improve the prediction of the zero-strength layer with increased load sharing and redistribution capacities. It is also important to investigate the effects of stress concentrations in the adhesive layers due to timber cracks propagating into the adhesive layer and local defect effects. As always with a variable material such as timber, additional testing is always recommended for repeatability and to decrease uncertainties in results.

\subsubsection{Practitioner Recommendations}

Practitioners are encouraged to continue to learn about the state-of-the-art in timber research, and to utilize the model development described herein to evaluate the applicability of structural models used for timber systems. It is clear that these models still require further development, and should be used with caution and an understanding of the limitations.

Additionally, practitioners should proceed with caution in using the standard recommended char rates, zero-strength layer and sacrificial char methodology. Although the results here are quite conservative in recommendations for an increased zero-strength layer, these results should be regarded as cautionary to designers for critical exposed structural 
elements using the tested types of adhesive. Furthermore, newer adhesives should be explored such as the new silicone-based adhesives under testing at NIST.

It is recommended that a new standardized test be developed for timber adhesives which quantifies the performance beyond the char layer in burnt engineered timber so that individual adhesives may be evaluated.

\subsection{Closing Remarks}

In order to implement innovative structures like PT timber, it is important to have a thorough understanding of the performance of the structure as a whole and the material at a more detailed level. The work performed in this thesis was a step towards rounding out the available knowledge on both of these levels. PT timber offers incredible benefits to the Canadian building industry including structural efficiency and sustainability, and it is hoped that the results achieved herein may be used by likeminded researchers and practitioners to safely and knowledgably implement PT timber structures in Canada. 


\section{References}

ANSI (American National Standards Institute) and APA (the Engineered Wood Association). (2008). ANSI 405: Standard for Adhesives for Use in Structural Glued Laminated Timber. Washington: ANSI/APA.

ANSYS Inc. (2016a). ANSYS Mechanical APDL Coupled-Field Analysis Guide. Help System, ANSYS, Inc., Canonsburg, PA USA.

ANSYS Inc. (2016b). Section 3.4.4 - Generalized Hill. ANSYS Mechanical APDL Materials Reference. Help System, ANSYS, Inc., Canonsburg, PA USA.

Ardalany, M., Deam, B., Fragiacomo, M., \& Crews, K. I. (2011). Tension Perpendicular to Grain Strength of Wood, Laminated Veneer Lumber (LVL), and Cross-Banded LVL (LVL-C). Proceedings of the 21st Australasian Conference on the Mechanics of Structures and Materials, pp. 891-896.

ASTM (American Society for Testing and Materials). (2008). ASTM-D905: Standard Test Method for Strength Properties of Adhesive Bonds in Shear by Compression Loading. Pennsylvania: ASTM International.

ASTM (American Society for Testing and Materials). (2016a). ASTM-D7247: Standard Test Method for Evaluating the Shear Strength of Adhesive Bonds in Laminated Wood Products at Elevated Temperatures. Pennsylvania: ASTM International.

ASTM (American Society for Testing and Materials). (2016b). ASTM-E1354: Standard Test Method for Heat and Visible Smoke Release Rates for Materials and Products Using an Oxygen Consumption Calorimeter. Pennsylvania: ASTM International. 
Babrauskas, V. (2001). Ignition of Wood: A Review of the State of the Art. Interflam, Interscience Communications Ltd., London, pp. 71-88.

Benzeggagh, M. L. and Kenane, M. (1996). Measurement of Mixed-Mode Delamination Fracture Toughness of Unidirectional Glass/Epoxy Composites with Mixed-Mode Bending Apparatus. Composites Science and Technology 56, pp. 439-449.

Bisby, L., Gales, J. and Maluk, C. (2013). A contemporary review of large-scale nonstandard structural fire testing. Fire Science Reviews, 2(1), pp.1-27.

Buchanan, A. H., Abu, A. (2017). Timber Structures, Structural Design for Fire Safety, 2nd ed., pp. 257-297.

Burgess, I., Davison, B., Huang, S. S., Plank, R. (2016). Structural Fire Engineering Research at the University Of Sheffield - Vulcan. The University of Sheffield, Sheffield, England.

Cachim, P. B., and Franssen, J. M. (2009). Comparison between the charring rate model and the conductive model of Eurocode 5. Fire and Materials, vol. 33, pp. 129-43. doi:10.1002/fam.985

Carter Holt Harvey Group. (2010). Designing in the Deep End: Diocesan Aquatic Centre. Carter Holt Harvey Group Ltd. FutureBuild LVL hySPAN: Case Studies, Available: https://www.chhwoodproducts.co.nz/hyspan/

CEN (European Committee for Standardization). (2004a). Part 1-2: General - Structural Fire Design, Eurocode 5: Design of Timber Structures. British Standards Institute, London, England, pp. $20-28$. 
CEN (European Committee for Standardization). (2004b). Annex B: (informative) Advanced calculation methods, Part 1-2: General - Structural Fire Design, Eurocode 5: Design of Timber Structures. British Standards Institute, London, England, pp. $48-51$.

CEN (European Committee for Standardization). (2004c). Section 3.4: Thermal elongation of reinforcing and prestressing steel, Part 1-2: General rules - Structural Fire Design, Eurocode 2: Design of Concrete Structures. British Standards Institute, London, England, pp. $28-29$.

Clauß, S., Joscak, M., Niemz, P. (2011). Thermal stability of glued wood joints measured by shear tests. European Journal of Wood and Wood Products 69, Springer-Verlag, pp. 101 - 111. doi: 10.1007/s00107-010-0411-4

COST (European Cooperation in Science and Technology). (2014). Fire safe use of biobased building products, FPS COST Action FP1404. COST Association, Brussels, Belgium. Available: http://www.cost.eu/COST_Actions/fps/FP1404

Costello, R. S. (2013). The Fire Performance of Post-Tensioned Timber Buildings. Christchurch, NZ: University of Canterbury.

CSA (Canadian Standards Association). (2006). CSA-O177: Qualification Code for Manufacturers of Structural Glued-Laminated Timber. Toronto: CSA Group.

CTBUH (Council on Tall Buildings and Urban Habitat). (2017). Tall Timber: A Global Audit, in Tall Buildings in Numbers. CTBUH Journal, 2017 Issue II, pp. 47 - 49. Available: http://global.ctbuh.org/resources/papers/3350-TBIN.pdf 
Dassault Systèmes. (2012). Section 6.5.2 - Uncoupled Heat Transfer Analysis: Elements. Abaqus User's Manual Volume II: Analysis. Dassault Systèmes Simulia Corp., Providence, RI, USA.

DIANA FEA. (2017a). History of DIANA FEA BV. Retrieved August 4, 2017, from https://dianafea.com/History

DIANA FEA. (2017b). 25.2 - Staggered Solution with Temperature. DIANA-10.2 User's Manual - Analysis Procedures, 1st ed. DIANA FEA B.V., Delft, Netherlands.

DiDomizio, M. J., Mulherin, P., and Weckman, E. J. (2016). Ignition of wood under timevarying radiant exposures. Fire Safety Journal 82, pp. $131-144$. doi:10.1016/j.firesaf.2016.02.002

Emberley, R., Nicolaidis, A., Fernando, D., Torero, J. L. (2016). Changing Failure Modes of Cross-Laminated Timber. Structures in Fire, Proceedings of the 9th international conference, New Jersey, USA, pp. $643-649$.

ETH Zürich. (2015). Laboratory for sustainable construction. ETH Zürich Press Release. Available: https://www.ethz.ch/en/news-and-events/eth-news/news/2015/06/mmhouse-of-natural-resources.html

Frangi, A., Fontana, M. and Mischler, A. (2004). Shear behaviour of bond lines in glued laminated timber beams at high temperatures. Wood Science and Technology 38, pp. 119 - 126. doi:10.1007/s00226-004-0223-y

Franssen, J. M. (2005). SAFIR: A Thermal/Structural Program for Modeling Structures Under Fire. Engineering Journal, pp. 143-158. 
Franssen, J. M. and Gernay, T. (2017). Modeling structures in fire with SAFIR®: theoretical background and capabilities. Journal of Structural Fire Engineering Vol. 8 No. 3, pp. 300-323. doi: 10.1108/JSFE-07-2016-0010

Fredlund, B. (1993). Modelling of Heat and Mass Transfer in Wood Structures during Fire, Fire Safety Journal, vol. 20, pp. 39-69.

Gales, J. and Green, M. (2015) Optical Characterization of High Temperature Deformation in Novel Structural Materials. Proceedings of the 14th International Conference on Fire and Materials, San Francisco, USA, pp. $626-640$.

Gales, J., Bisby, L. and Stratford, T. (2012). New Parameters to Describe HighTemperature Deformation of Prestressing Steel Determined Using Digital Image Correlation. Structural Engineering International, 4, pp. $476-486$. doi:10.2749/101686612X13363929517730

Hadden, R. M., Bartlett, A. I., Hidalgo, J. P., Santamaria, S., Wiesner, F., Bisby, L. A., Deeny, S., Lane, B. (2017). Effects of exposed cross laminated timber on compartment fire dynamics. Fire Safety Journal 91, pp. $480-489$.

Hajiloo, H., Green, M. F., Gales, J. (2018). Mechanical properties of GFRP reinforcing bars at high temperatures. Construction and Building Materials 162, pp. $142-154$. doi: 10.1016/j.conbuildmat.2017.12.025

Hankinson, R. L. (1921). “Investigation of Crushing Strength of Spruce at Varying Angles to Grain.” Air Service Information Circular No. 259, U.S. Air Service. 
Holden, T., Devereux, C., Haydon, S., Buchan, A., and Pampanin, S. (2016). NMIT Arts \& Media Building-Innovative structural design of a three storey post-tensioned timber building. Case Studies in Structural Engineering, Volume 6, December 2016, pp. 76 - 83. doi: https://doi.org/10.1016/j.csse.2016.06.003

Hopkin, D. J., El-Rimawi, J., Silberschmidt, V., and Lennon, T. (2011). An effective thermal property framework for softwood in parametric design fires: Comparison of the Eurocode 5 parametric charring approach and advanced calculation models, Construction and Building Materials, vol. 25, pp. 2584 - 2595. doi:10.1016/j.conbuildmat.2010.12.002

ICOMOS. (1993). World Heritage List: Horyu-ji, Advisory Body Evaluation No. 660. Available: https://whc.unesco.org/en/list/660/documents/

Ingberg, S. (1919). Fire Tests of Building Columns. Associated Factory Mutual Insurance Companies, The National Board of Fire Underwriters and The Bureau of Standards Department of Commerce, Chicago, Illinois, USA.

Iqbal, A. and Popovski, M. (2017). Post-tensioned Timber Structure: A Novel Solution for Multi-storied Buildings. Canadian Civil Engineer Fall 2017. Canadian Society for Civil Engineering, pp. $17-20$.

ISO (International Organization for Standardization). (2014). ISO 834-10. Fire Resistance Tests: Elements of Building Construction, International Organization for Standardization, Geneva, Switzerland.

Jeanneret, C., Smith, M., and Gales, J. 2017. Fire safety towards enabling timber structures in Canada. Applications of Structural Fire Engineering. 
Kirk Roberts Consulting Engineers. (2014). Case Study: Young Hunter House. Available: https://www.kirkroberts.co.nz/images/projects/young-hunterhouse/young_hunter_house_brochure.pdf

Kollmann, F. F. P. and Côté, W. A. (1968). Principles of Wood Science and Technology 1: Solid Wood. New York, Springer-Verlag Inc., p. 326.

König, J. (2005). Structural fire design according to Eurocode 5 - design rules and their background. Fire and Materials, vol. 29, pp. 147-163. doi:10.1002/fam.873

König, J. (2006). Effective thermal actions and thermal properties of timber members in natural fires. Fire and Materials, vol. 30, pp. 51-63. doi:10.1002/fam.898

Kováčiková, J., Ekevad, M., Ivánková, O. and Berg, S. (2016). Finite Element Analysis of Timber Beams With Flaws. ECCOMAS Congress 2016, Crete Island, Greece.

LaMalva, K. J. (ed). (2018). Chapter 11: Structural Acceptance Criteria - 11.5: Timber, in Structural Fire Engineering: Manual of Practice. ASCE/SEI Fire Protection Committee, Structural Engineering Institute, American Society of Civil Engineers.

Lange, D. Boström, L., Schmid, J., and Albrektsson, J. (2015). The Reduced Cross Section Method Applied to Glulam Timber Exposed to Non-standard Fire Curves. Fire Technology 51, Springer Science, New York, USA, pp. 1311 - 1340. doi:10.1007/s10694-015-0485-y

Lewis, T. H. (1865). Fire-Proof Materials and Construction. Read at the Ordinary General Meeting of the Royal Institute of British Architects, April 3, 1865. Sessional Papers of the Royal Institute of British Architects, London, England. 
Livermore Software Technology Corporation. (2011). LS_Dyna. Retrieved August 4, 2017, from http://www.lstc.com/products/ls-dyna

McGechie, M. (2011). Innovative post-tensioned Expan timber building. Build 123 April/May 2011. Available: https://www.buildmagazine.org.nz/assets/PDF/Build123-76-BRANZ-refurbishment-Innovative-Post-tensioned-Expan-TimberBuilding.pdf

Menis, A. (2012). Fire resistance of Laminated Veneer Lumber (LVL) and CrossLaminated Timber (XLAM) elements. Cagliari, Italy: Università Degli Studi di Cagliari.

Nicolaidis, A., Emberley, R., Fernando, D., Torero, J. L. (2016). Thermally Driven Failure Mode Changes in Bonded Timber Joints. Proceedings of World Conference on Timber Engineering, WCTE 2016, Vienna, Austria.

NIST (National Institute of Standards and Technology). (2007). Voluntary Product Standard PS1: Structural Plywood. Maryland: NIST.

Nordic Wood Structures. (2015). Technical Note S101: Design Properties, Nordic Lam. Nordic Structures, $\quad$ Québec, 1 pp. Available: https://www.nordic.ca/data/files/datasheet/file/T-S01_ePropertiesNordicLam.pdf

NRC (National Research Council Canada). (2015). National Building Code of Canada. Canadian Commission on Building and Fire Codes.

NRC (National Research Council Canada). (2018). Encapsulated Mass Timber Construction, Public review on proposed changes to Codes Canada publications - 
Fall 2017, Codes Canada, National Research Council of Canada (https://www.nrccnrc.gc.ca/eng/solutions/advisory/codes_centre/public_review/2017.html).

NZ Wood. (2013). Case studies: EXPAN Building 'The safest place on Campus'. NZ Wood, Wellington, NZ. Accessible: http://www.nzwood.co.nz/case-studies/expanbuilding-the-safest-place-on-campus/

Ontario Ministry of Municipal Affairs and Housing (MMAH). (2012). Ontario Building Code, O. Reg. 332/12, Queen's Printer for Ontario.

Palermo, A., Pampanin, S., Buchanan, A., \& Newcombe, M. (2005). Seismic design of multi-storey buildings using Laminated Veneer Lumber (LVL). Proceedings of the New Zealand Society for Earthquake Engineering Conference, Paper 14.

Pizzo, B., Lavisci, P., Misani, C., Triboulot, P., Macchioni, N. (2003). Measuring the shear strength ratio of glued joints within the same specimen. European Journal of Wood and Wood Products (Holz als Roh- und Werkstoff) 61, pp. 273 - 280. doi: $10.1007 / \mathrm{s} 00107-003-0386-5$

Quiquero, H., and Gales, J. (2016). Behaviour of Fire-Damaged Engineered Timber Beams. Canadian Society for Civil Engineering, 5th International Structural Specialty Conference, London, Canada, STR-138.

Quiquero, H. and Gales, J. (2017). Comparing Timber Adhesive Shear Strength Properties after Fire Damage. Proceedings of the $15^{\text {th }}$ International Fire and Materials Conference 2017, San Francisco, USA, pp. 556 - 566. 
Quiquero, H., Gales, J., and Hadjisophocleous, G., et al. (2016). Behaviour of Char Layer in Fire-Damaged Box Section Timber Beams. Interflam 2016, England, pp. 1063 1074.

Reiterer, A., Sinn, G., and Stanzl-Tschegg, S. E. (2002). Fracture characteristics of different wood species under mode I loading perpendicular to the grain. Materials Science and Engineering A332, pp. 29-36.

Reszka, P. and Torero, J. L. (2008). In-depth temperature measurements in wood exposed to intense radiant energy. Experimental Thermal and Fluid Science 32, pp. 1405 1411. doi:10.1016/j.expthermflusci.2007.11.014

Richter, F. and Rein, G. (2018). The Role of Chemistry in Predicting the Charring Rates under Realistic Fire Conditions. 12 ${ }^{\text {th }}$ International Performance-Based Codes and Fire Safety Design Methods, Honolulu, USA.

Roberts, A. F. (1971). The heat of reaction during the pyrolysis of wood. Combustion and Flame, vol. 17, pp. 79-86.

Schaffer, E. L. (1967). Charring Rate of Selected Woods - Transverse to Grain. Research Paper FPL 69, U.S. Department of Agriculture, Forest Service, Forest Products Laboratory, Madison, Wisconsin, USA. 22pp.

Schaffer, E. L., Marx, C. M., Bender, D. A., and Woeste, F. E. (1986). Strength validation and fire endurance of glued-laminated timber beams. Research Paper FPL 467, U.S. Department of Agriculture, Forest Service, Forest Products Laboratory, Madison, Wisconsin, USA.16pp. 
Smith, H. K. M., Reid, E. R. E., Beatty, A. A., Stratford, T. \& Bisby, L. A. (2011). Shear strength of concrete at elevated temperature in Applications of Structural Fire Engineering: Focusing on utilisation of software tools, Prague, pp. $133-138$.

Spearpoint, M. J. and Quintiere, J. G. (2001). Predicting the piloted ignition of wood in the cone calorimeter using an integral model - effect of species, grain orientation and heat flux. Fire Safety Journal 36, pp. 391 - 415. doi:10.1016/S0379$7112(00) 00055-2$

Spellman, P. M. (2012). The Fire Performance of Post-Tensioned Timber Beams. Christchurch, NZ: University of Canterbury.

Stanier, S.A., Blaber, J., Take, W.A. and White, D.J. (2015). Improved image-based deformation measurement for geotechnical applications. Canadian Geotechnical Journal, 53(5), pp. 727 - 739. doi:10.1139/cgj-2015-0253

Su J., Lafrance P.S., Hoehler M., and Bundy M. (2018). Fire Safety Challenges of Tall Wood Buildings- Phase 2: Task $2 \& 3$ - Cross Laminated Timber Compartment Fire Tests. FPRF, $397 \mathrm{pp}$.

Thomas, P. H. (1995). The growth of fire - from ignition to full involvement, in. Combustion Fundamentals of Fire, Cox (ed). Academic Press, England, pp. 273328.

Van Beerschoten, W. A. (2013). Structural Performance of Post-tensioned Timber Frames under Gravity Loading. Christchurch, NZ: University of Canterbury. 
Vulcan Solutions. (n.d.). Software. Retrieved August 4, 2017, from http://www.vulcansolutions.com/?page_id=201

Werther, N., O'Neill, J. W., Spellman, P. M., Abu, A. K., Moss, P. J., Buchanan, A. H., \& Winter, S. (2012). Parametric Study of Modelling Structural Timber in Fire with Different Software Packages. Proceedings of the 7th International Conference on Structures in Fire, pp. 427-436.

White, R. H. (2000). Charring of Composite Timber Products. Proceedings of Wood and Fire Safety 2000 Part 1, $4^{\text {th }}$ International Scientific Conference, Slovakia, pp. 353363.

Xiea, D., \& Biggers, S. B. (2006). Progressive crack growth analysis using interface element based on the virtual crack closure technique. Finite Elements in Analysis and Design 42, pp. 977-984.

XLam Cross Laminated Timber Panels. (2017). XLam Rocking Shear Walls Withstand Kaikoura Earthquake. EBOSSNOW Case Studies. Available: https://www.eboss.co.nz/ebossnow/xlam-cross-laminated-timber-panels-rockingshear-walls-seismic-performance

Yeh, B., Herzog, B. and Williamson, T. G. (2005). Adhesive Performance at Elevated Temperatures for Engineered Wood Products. International Council for Research and Innovation in Building and Construction. Available: https://www.apawood.org/Data/Sites/1/documents/technicalresearch/paper-2005cib-w18---adhesive-heat-durability.pdf 


\section{Appendix A - Developing Fire Safety Engineering as a}

\section{Practice in Canada}

\section{A.1 Introduction}

The goal of fire safety engineering (FSE) has always been to design an optimized strategy which provides the necessary human safety and property protection. In Canada, our engineering community does not need to look very far to see the challenges our society is currently facing with respect to fire safety related to our infrastructure. In the last five years there have been multiple severe fires, including the Fort McMurray wild fire, the L'Isle Verte care home, and Kingston's 2013 city conflagration. In particular, a surge of complex structures has become the norm as our society is rapidly undergoing a significant shift to urban populace, relying on novel building technologies and material optimization strategies which are pushing the boundaries of our existing building codes (see Figure A.1). These issues have necessitated a more holistic view of fire safety which warrants a discussion of our treatment of FSE in Canada.

The purpose of this state-of-the-art study is to create awareness of novel FSE concepts used internationally and discuss potential growth for the Canadian design practice. The authors intend this review to provide a well-rounded background on FSE for the Canadian practitioner, in order to facilitate a more commonplace holistic understanding of the importance and potential of fire safety engineering. This manuscript serves to begin that discussion.

Herein, an introduction to performance-based fire design (PBFD) is provided. A review of the fundamental and supporting FSE concepts, strategies and benefits as seen historically 
and internationally is provided. The study aims to provide a review of exemplar FSE projects developed at leading international engineering firms. A summary of some bestsuited project types is provided. Finally, an insight into the future of FSE sheds light on the forthcoming opportunities for Canadian practitioners in this field.

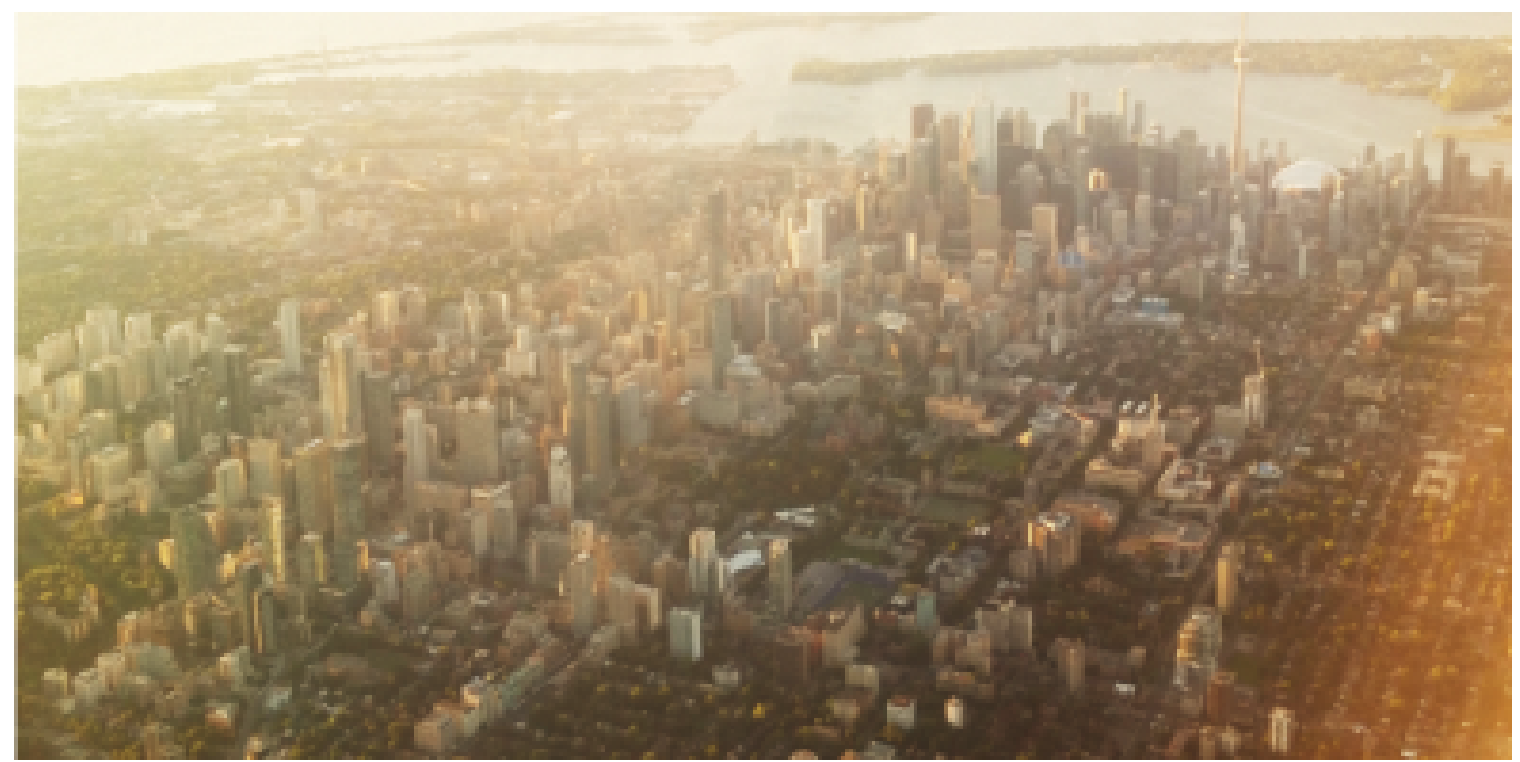

Figure A.1: In Toronto alone, there are now over 25 complex and highly optimized buildings that are 50 stories or higher. Nearly 15 are under construction and nearly 30 are approved and/or proposed. Additionally, to accommodate the urbanization, significant and complex infrastructure is proposed. Each engineering project may have the potential for application of advanced FSE to facilitate safe best practice benefits seen globally. (Author's photo).

\section{A.2 Background}

Current practice related to fire safety strategies relies primarily on architects or code consultants who either apply prescriptive code clauses or attempt to demonstrate equivalency to the prescriptive solutions. Mechanical and electrical engineers may design HVAC or sprinkler equipment in line with the requirements of the code while structural engineers demonstrate their design has the required fire resistance ratings, but the architect is still primarily responsible for overseeing implementation of these elements in the overall 
building with code consultants on the project team being responsible for interpretation of code clause intents. It can be seen internationally that there is a trend towards using more engineering based approaches to demonstrate safety as opposed to simply complying with prescriptive code provisions. As FSE is not often seen in practice in Canada, this section aims to provide the reader with sufficient background on its evolution. A description of PBFD within the Canadian context is given as it is the basis of Fire Safety Engineering. Additionally, information on the condition of FSE education and an introduction to FSE practice around the globe are provided.

\section{A.2.1 Performance-Based Fire Design}

With prescriptive-based fire design, infrastructure is generally understood to be safe. However the degree of safety provided may not be rationally understood in all cases, and may vary from application to application. PBFD is an alternative method of designing to meet the objectives of the building code, without expressly using the prescriptive clauses and limiting requirements. Speaking specifically to fire, the code clauses developed are allencompassing, applicable to a wide range of structure sizes and functionalities, which could be inhibiting in a unique design scenario. In many cases, the fire safety strategies outlined in the code could be overly conservative and may result in highly overdesigned fire safety strategies, causing unnecessary costs to the client and users. These costs may come in the form of excessive fire protection, loss of profitable floor area, or sacrifice of original architectural vision. In other cases, with detailed analysis, the prescriptive code provisions may prove to be insufficient for increasingly complex and unique buildings. However, currently a high level of fire analysis and modelling to support an alternative design is required for regulatory approval. As best said in 1995 by pioneering PBFD 
international experts Margaret Law and Paula Beever: "The desire of regulators to have simple rules and tests for administrative convenience contrasts with the need of designers to have maximum flexibility in order to arrive at optimum solutions. The magic numbers embodied in regulations are accepted without question, while any engineering solution is subject to a disproportionately high standard of proof" (Law and Beever 1994).

The use of PBFD solutions requires a deep understanding of FSE. Careful attention to the fire protection effectiveness during the design, construction, and management phases is critical. Any mistake or omission could result in the failure of the whole fire protection strategy. The British Standards Institution has developed BS 9999, a code for PBFD (BSI 2008), in which it is stated: "It has been found in practice that designs can frequently be compromised due to incorrect or poor installation, substituted materials or products, missing materials or products, lack of integration of active systems, inadequate inspection, lack of full commissioning, abuse during normal use of the building, inadequate maintenance and/or testing, and problems resulting from inadequate management documentation and training" (BSI 2008). Due to this fact, it is crucial to develop a comprehensive design that is integrated flawlessly within the building systems. There are limited technical resources available for PBFD (even internationally), and a large amount of learning from past case studies must be done - particularly for new practitioners eager to pursue this area - as has been compiled in this manuscript. Of the existing literature on PBFD, readers are encouraged to consult 'Performance-Based Fire Engineering of Structures' by Wang et al. (2013) and 'Structural Design for Fire Safety, Second Edition' by Buchanan and Abu (2017) for education on performing more advanced design and material specific techniques, which are beyond the scope of this report to detail in text. 
Currently in Canada, with the introduction of the objective-based code in 2005 that contained the Alternative Solutions clause, designers had more freedom to deviate from the code prescribed solutions. Part 1 in Division A in the National Building Code of Canada (NRC 2015) outlines the use of Alternative Solutions for code compliance, and Part 2 in Division $\mathrm{C}$ gives the administrative framework for creating an Alternative Solution proposal. Clause 1.2.1.1, sentence 1(b) states that "Compliance with Division B shall be achieved by using Alternative Solutions that will achieve the level of performance required by the applicable acceptable solutions..." (NRC 2015). Each code clause that may be adopted to an equivalent solution is linked to Functional Statements and Objectives through the MMAH Supplementary Standard SA-1, that explain the underlying requirement of a code provision for which an alternate solution may be developed.

The first design examples in Canada which utilised this procedure have referenced a PBFD guideline published by the Society of Fire Protection Engineers (SFPE 2000), such as the Nova Scotia Community College (Bartlett 2005) and the Citadel High School (Ponto 2006). The general approach for these case studies was to use Fire Dynamics Simulator (FDS) to calculate expected temperatures in the exposed structural steel based on a realistic fire scenario and show they were below the limiting temperature prescribed by the standard fire test, CAN/ULC-S101 (ULC 2014). That same trend has continued in subsequent Canadian case studies, which appear to be heavily reliant on FDS for temperature calculations but do not consider, or at least discuss in the literature, whole building structural response to fire. Such consideration of the structural response of the entire building is best practice and is critical to complete a holistic engineering solution, and is typically done on accepted FSE projects internationally. Compared to international 
applications of structural fire engineering discussed later, the available Canadian case studies are quite limited. If methods other than demonstrating a limiting temperature in structural elements are being implemented in practice, they need to be shared in technical publications to invite critique, share knowledge, and demonstrate the benefits of using FSE in practice.

Other applications of the Alternative Solutions clause in Canada are related to human egress during fire events (RJ Bartlett Engineering Ltd. 2014), as well as in certain highrise applications to demonstrate overall equivalency to a high-rise comprised of acceptable solutions (Harmsworth and Dagenais 2014). When Canada transitioned to the objectivebased code in 2005, it was said that "there is a general trend towards performance-based codes and objective-based codes can help guide the way along that path" (Bergeron et al. 2004). Alternative solutions being implemented in Canada are trending towards PBFD with the specific Authority Having Jurisdiction (AHJ) often determining the extent to which engineering based approaches are allowed. This demonstrates the importance of educating authorities in delivering FSE in Canada.

\section{A.2.2 Education}

In Europe, which is arguably a leader within FSE, there exists a colloquium of universities that provide engineers the necessary background to practice FSE within the context of their countries building codes. For example, the joint International Master of Science in Fire Safety Engineering (IMFSE 2016) involves a co-operation of universities from Belgium, Sweden, and the United Kingdom. The programme extends to offering trainees experiences in Switzerland, Australia, and the United States. The curriculum encompasses fundamentals in fire dynamics, risk analysis, human behaviour, structural design and a 
short research thesis (in that order to minimize course redundancy). The program, however, is rather inaccessible to Canadians as it would demand they train abroad which is not conventionally feasible to all interested practitioners (especially those currently practicing).

To train fire safety engineers in Canada, there currently are three institutions offering undergraduate and graduate courses in fire safety engineering: Carleton University, the University of Waterloo and York University. These courses primarily include fire dynamics, and structural behaviour (fire resistance) in fire. Human behaviour in fire was recently introduced to the curriculum in 2015 at Carleton University and in 2018 at the University of Waterloo and York University; prior to this no formal education existed in Canada on this topic since 2008. Each university gives the option to the practitioner to undertake a research based thesis. These educational facilities offer practitioners the option to study via online lectures which is highly adaptable to the desires of practitioners whom may already be working. Structural behaviour is taught at both institutions yearly using the first edition text Structural Design for Fire Safety by Buchanan (2002). This text is beginning to age relative to international practice, research, and computational abilities. Notably, the document is written before the events of September $11^{\text {th }}$ in 2001 which renewed North American research and education in fire safety. A second edition of the text by Buchanan and Abu (2017) introduces new material in light of research done over the past fifteen years including best-practice guidance for designers and advanced calculation methods. It is anticipated that the structural fire courses will require a curriculum update to match the scope and content of this updated text should they specify the second edition in their curriculum. As well, the structural fire course, and the fire safety engineering courses 
in general, have a lack of design problems that are provided in international educational experiences. Such problems would train students to apply the knowledge fundamentals to solve real-world problems, which is crucial in professional academic programs, to train future engineers to provide PBFD (Woodrow et al. 2013).

While the Canadian curriculum certainly has strengths, this critical review suggests there is room for improvement when comparing to our international peers. Such improvements may include removing redundancy from the courses offered, focusing more on firstprinciples of fire safety, introducing more design-type problems, and increasing the collaboration between industry and academia. These characteristics of a successful fire safety engineering program have been demonstrated in-part internationally (Richardson 2003) and have been shown to lead to a more developed fire safety profession with many consultancies actively participating and a plethora of innovative case studies to learn from, as will be discussed below. A key challenge that still exists is engaging AHJ in FSE education such that proposed designs can be competently reviewed, critiqued, and approved.

\section{A.2.3 Practice}

In order to implement FSE services at a consultancy office, it is important to learn from the successes and failures of previously established companies. Major firms that have consistently published works on their projects (in the form of journal articles, magazine articles and conference proceedings) allow the FSE profession to be developed and validated in the public realm. It is the opinion of the authors that in order for FSE professionals to be recognized as competent engineers, it is essential that design solutions be published for the community to learn, and critique. Professionals must practice within 
the public view, under regulating body and peer review, if the field is to advance and provide opportunity for the safe adaptation of past FSE designs into new projects (Torero 2012). Engineering consultants such as Arup, BuroHappold, and Trenton Fire (etc.) have provided fire engineering services on hundreds of international projects and this has allowed them to be part of the design team for some of the most innovative and iconic buildings in the world. These companies have widely published their FSE strategies on various projects and can be viewed as exemplars for Canadian companies to model if not emulate.

Arup progressed the fire engineering service in the mid-1970s when Margaret Law was hired. At this time, prescriptive code provisions for fire-related requirements were also being developed, in a similar manner to current Canadian building codes. The new FSE department struggled with arbitrarily increasing fire protection requirements and size limitations from the government, which were seen as largely political but unsupported by research (Law 1986). Law proposed the need for research to prove that for more complex and innovative buildings, a unique fire engineering design can be developed for each case which will result in a more economical solution, directly proportionate to the people and property at risk (Law 1986). The period between when Arup established their FSE group to the time that they were able to have their first PBFD project approved was over 20 years long, when the London City Hall building was approved. For this project the structural fire protection was reduced using a time-equivalency method, and smoke management and evacuation procedures were developed for the mixed occupancy of general public and office staff (O’Meagher and Ferguson 2003). This first approach, the time-equivalency method, was innovative at the time of the project but has since become antiquated and is 
no longer recommended as best practice, whereas smoke management and egress modelling have become large parts of modern FSE. Later, in 2004, the fire group had developed and applied what is believed to be the first ever thermo-mechanical analysis and design solution in London. On the Plantation Place South development (Goymour 2005), they were able to eliminate structural fire protection on several steel beams, relying on tensile membrane action as validated by the (then) recent Cardington fire tests (Usami et al. 2000).

After the founding of this FSE group in the UK and several decades of work to establish the value of the service, other firms began offering fire engineering services including BuroHappold and Trenton Fire (as a division of Butler \& Young) in the mid-1990s (Green and Joinson 2010, Trenton Fire 2016). Subsequently, FSE has become widely accepted in several countries in Europe, Asia and the Middle East. For Canada, it may take effort to establish inaugural acceptances or a standard of practice, but should not require the same amount of time and strife as was demanded in the 1970 s - if these companies are used as exemplar case studies. Already, branches of large firms which offer FSE internationally have begun to introduce FSE concepts in Canada. However, as aforementioned, these firms must emulate these UK-based companies and publish technical reports in the public realm if they are completing complex FSE projects, in order for the Canadian industry to expedite the AHJ acceptance process.

\section{A.3 Design}

This section aims to provide the reader with a solid foundation of FSE concepts through outlining the fundamental theories and common effective strategies which have been developed and applied by leading international universities and firms. Furthermore, a list 
of best-suited projects and case studies are given to demonstrate where FSE may be employed in Canada.

\section{A.3.1 Fundamentals}

In order to use FSE to produce PBFD solutions, there are several fundamental theories which are applied to each unique project. An understanding of these FSE concepts is the baseline requirement for the ability to provide fire engineering services to clients.

\section{A.3.1.1 Design Fires}

Under the current code provisions, all construction materials and components are tested with a Standard Fire Test (ISO 2014). This testing is typically done in a furnace with a standard time-temperature curve which was developed early in the 20 th century by the ASTM, in which the temperature rises to almost $700^{\circ} \mathrm{C}$ in the first 10 minutes. By the 60 minute mark, the temperature in the fire compartment is over $925^{\circ} \mathrm{C}$, and continues to rise almost linearly but indefinitely. The time until which a component fails in this test is its "Fire-Resistance Rating," which is qualified as a loss of structural integrity, or too much heat or smoke penetrating the component. The test was first used in June 1917 as part of a comparative index of ratings for concrete, steel and timber columns which were placed under a service load and standard heating. In the test the isolated materials were then ranked by time of failure. The appropriateness of these tests was further limited by the difficulties

of manual temperature control, especially with timber specimens which may give off their own heat (Ingberg 1919). It is additionally important to note that components tested with this methodology are then incorporated into building systems in which they interact with other components. These interactions can then lead to unique failure mechanisms which can only be observed if an analysis of the entire structure, or a part of a structure with 
appropriate boundary conditions, is performed. The test would later evolve to include the concepts of restraint to represent these boundary conditions, but still cannot accurately resemble true structural supports which are highly variable.

Additionally, this Standard Fire as defined by temperature alone is not representative of a real fire and has no physical meaning in reality, other than providing a standardized temperature exposure (Bisby et al. 2013). Generally, building fires can reach much higher temperatures in varying amounts of time and will enter into a decay phase after either the available fuel or oxygen is consumed. In the standard test the fire is uniform along the specimen, whereas in reality the fire may not be. Recent testing in the UK has indicated that for some structures, non-uniform (or localized) fire exposure may be more critical and a better representation of real fire dynamics (Gales et al. 2016). Several highly-variable factors contribute to the way a fire will burn in a structure. The major conditions that affect fire burning times, temperatures, and behavior include the type and amount of fuel present, building geometry and compartment thermal properties, and the ventilation rate based on openings. In the majority of cases, a highly ventilated fire is desired because hot, toxic smoke is removed and the temperature of structural elements lags behind leading to a shorter duration of heating in the members.

In order to appreciate the significance of design fire definition, it is important to understand a brief history of how fire behaviour knowledge has developed. Due to the limited understanding of fire dynamics and structural mechanics at elevated temperatures in the 1920 s, FSE at that time relied on equivalency based rules (now known to be inadequate to represent reality) to the Standard Fire. The methodology was developed by Simon Ingberg, and was meant to be representative of a fire from start to burn-out (Ingberg 1928). In 1967, 
the Fire Research Station in Boreham, UK held an important fire symposium. Here, it became evident that these prescriptive rules make it difficult to quantify just how much safety or little safety can be provided for real fires and complex assemblies. Prominent attendees including Margaret Law (then of the Fire Research Station) and Tibor Harmathy of the National Research Council Canada (NRCC) remarked on the lack of accurate tools and experiments available to quantify this concept (HMSO 1968). Over the following decades, Harmathy would continue efforts towards understanding fire dynamics and developing tools for fire modelling. He performed experiments in which he studied underand over-ventilated fires at the NRCC and created numerous empirical approximations (Harmathy 1972). Others would continue to study these aspects internationally, and the reader is encouraged to consult the work of Torero et al. (2014) for a more contemporary review of the subject.

By the early 1990s, research facilities and consultancy companies began to study structural fires. This was mobilized by the Broadgate Fire, a composite steel-frame structure which caught fire during construction, prior to the application of fire protection (SCI 1991). A series of large-scale, real fire experiments were conducted on three structures - a composite steel frame, a concrete frame and a timber frame structure. The tests provided abundant insight into real structural behaviour, but yielded limited data for real fire modelling and verification. A subsequent set of similar tests were then performed at Cardington which focused on fire dynamics in the late 1990s.

Following these preliminary experiments, several researchers began to study realistic fire behaviour. Tests by Kirby began to illustrate unusual fire behavior in exceedingly large compartments (Kirby et al. 1999), and tests by Lennon in the early 2000s illustrated 
interesting dynamics associated with compartment boundaries and ventilation (Lennon 2003). The Dalmarnock Tests, a series of compartmentalized fires in a reinforced concrete tower complex, would follow in the mid-2000s led by the University of Edinburgh which provided additional scrutiny for our modern fire modelling tools (Stern-Gottfried et al. 2010), and today a series of large scale compartment tests are being performed by an international consortium funded through the EPSRC. The National Institute of Standards and Technology (NIST) has a fire testing facility in Maryland, USA in which numerous experiments have been carried out since 1975. The National Fire Research Laboratory (NFRL) expansion was completed in 2015 and the facility now has the capacity to perform experiments on full-scale structures in realistic fire conditions (Bundy et al. 2016).

Today, there are three accepted categories of realistic fires that may occur inside a building. The first is in a compartment such as enclosed offices or retail spaces, which burn very hot and fast in a high-ventilation situation and burn slow and long in a low-ventilation situation (Wang et al. 2013). The second possible fire scenario is in a compartment in which the fire has not reached flashover, and is considered as a localized fire plume. Finally, in a large open space such as an atrium or concourse, a contemporary proposed scenario is known as a Travelling Fire (which will be further discussed in proceeding sections). Evidently, there are a large number of different fire scenarios that may occur within a building. A deterministic approach to fire safety design considers several key "Design Fires" which are determined to be critical cases, and are then used to develop an appropriate fire strategy and protection scheme. 


\section{A.3.1.2 Hand Calculations \& Modelling}

After the Design Fires have been determined, hand calculations and modelling follow. Calculations are either done through spreadsheet and/ or validated computer software. The two major underlying numerical methodologies to be understood are finite element analysis (FEA) and computational fluid dynamics (CFD).

FEA is used in a variety of ways in a fire engineering analysis. Using software such as Vulcan (Vulcan Solutions 2016) or SAFIR (University of Liège 2016), the design fire parameters are input and the temperature histories of structural members in those fires may be determined. This is typically done by applying environmental time-temperature data as boundary conditions on an element cross-section. The temperature differential that transfers through the cross-section of each element is then calculated at each time step. FEA is also used subsequently to analyse the structural performance of the elements at these elevated temperatures with applied loading. In updated software such as an LSDYNA interface (Oasys Software 2016b), the thermal and subsequent structural analyses can be coupled into a single simulation encompassing all of the fire effects. Models created for these simulations should reflect non-linear and local plastic deformations and failures, and consider changing material properties at elevated temperatures.

CFD is typically used to model smoke generation and migration through a space or building, and can be used to design and justify smoke control system requirements or lack thereof. Based on the fire, materials and building geometry, the CFD analysis predicts how the hot and toxic smoke layer will develop which is a limiting factor in occupant egress safety. It can also determine the effects of smoke buoyancy and exhaust - critical parameters in the design of atria, stairwells and interconnected spaces. 
Another modelling tool which is currently under rapid research and development is software which models human behaviour and movement within a building. The analysis uses a combination of pedestrian flow information and contributions from randomized personalities in each individual "agent" (occupant in the simulation). The tools are being developed (such as MassMotion (Oasys Software 2016a and Bailey et al. 2008), Pathfinder, etc.) using extensive data observed from fire drills and from normal pedestrian movement, and each agent has their own agenda or priorities affecting their behaviour. This type of simulation can be used to determine problems in evacuation routes or justify code deviations in travel distances or exit widths. This has yet to be applied to FSE in Canada on a published and peer reviewed fire design, though its principles and benefits have been realised in other civil engineering fields such as transportation infrastructure (King et al. 2014).

\section{A.3.1.3 Design Strategy Implementation}

A vital component to a successful PBFD is working with key stakeholders and governing bodies from the very beginning. At the preliminary design phase of the project, fire engineering strategies should be planned and discussed with the client and their insurance provider to determine their business or property priorities, and with the authority having jurisdiction to ensure a smooth and timely approval process.

\section{A.3.2 Design Strategies}

There are three types of PBFD design measures which can be employed in a combination of strategies. These categories include Occupant Egress, Smoke Control and Flame Spread Containment, and Structural Fire Protection. Various strategies have been assembled after the review of numerous case studies from successful fire engineering projects. 


\section{A.3.2.1 Occupant Egress}

1. A direct way to demonstrate the safety of an occupant egress strategy is to compare the Required Safe Evacuation Time (RSET) for the occupants to exit to a safe area, versus the Available Safe Evacuation Time (ASET) before fire conditions are no longer tenable for human safety (Carfrae et al. 2011). This is typically done through occupant movement simulations or calculations, and smoke and heat development analyses. This can be used to justify longer travel distances or reduced exit widths for egress routes. Note that it has been observed that the width of the exit (and therefore the queueing time at the exits) usually governs the RSET rather than increased travel distances, where spaces are open and clear (Law 1986 and Carfrae et al. 2011).

2. There are several different evacuation strategies that can be developed for the building management. Phased evacuation is when only the zone in the building with immediate hazard is evacuated first, allowing for reduced demand on the egress routes and increased business continuity in the event of a false alarm. Horizontal evacuation can be used with fire separated zones, where the egress strategy is to move occupants into another safe compartment within the building. This reduces total evacuation times and again allows for business continuity. Egress elevators are another emerging strategy in which working elevators can be considered means of evacuation, especially for accessibility or tall building applications, but are still under much scrutiny in practical situations as generations of individuals have been trained not to utilize elevators in an emergency.

3. It is often justifiable and beneficial to design normal circulation routes as primary means of egress as it has been proven that occupants typically exit the building the way 
they entered in emergency scenarios, and alternate egress routes or stairwells are often overlooked (Dixon et al. 2010).

\section{A.3.2.2 Smoke Control \& Flame Spread Containment}

1. Smoke control is an effective way of increasing ASET through exhaust or pressurization systems. Exhaust systems can be implemented through natural or power ventilation, and may be validated using CFD analysis in complex cases. If designed properly, pressurization systems utilize high pressure zones within egress routes or firefighting shafts to induce air flow out of the zones and therefore inhibit infiltration of smoke into said zones. However, there is concern that the systems do not always perform as intended during design (Lay 2014) which demonstrates the importance of a holistic approach to FSE, and for designers to understand the limitations of the systems they implement.

2. A "cabin" methodology can be used to contain flame spread and smoke within one area, through the use of suppression systems and fire barriers. These areas are typically high-risk fire zones with large fuel loads, and can be compartmentalized through permanent walls or fire shutters, or open concept. In any situation, the cabin should be designed so that smoke and flames can be contained within that zone with a combination of automatic sprinklers, strategically placed barriers, and smoke exhaust vents or reservoirs (Carfrae et al. 2011).

3. The "island" methodology is another concept developed in which fire spread is contained simply through distance between combustible materials. This can be employed in large, open-concept areas such as concourses or waiting areas in transportation buildings, where there are very small amounts of combustibles. The heat 
release from a fire in one island of combustibles, or fuel, is determined considering the type of materials and ventilation. Next, an analysis is done to ensure the heat release is not sufficient to ignite another adjacent combustible island, therefore preventing the spread of the fire from its origin (Carfrae et al. 2011).

4. Various detection systems can be designed or specified to ensure a fire engineering strategy is well executed, and to aid in reducing RSET and increasing the amount of the ASET that occupants are able to use effectively (i.e. movement time may begin earlier). The choice of detection system is highly important, and the appropriate type of system varies in every building application. The varieties include smoke detectors, heat detectors (either sensing a rate of temperature rise or a given temperature threshold), and infrared flame detectors. "Double interlock" detection systems can also be employed which only activate minor alarms (rather than throughout the entire building) until a second detector or manual pull is triggered, reducing the likelihood of a false alarm. Avoiding false alarms is not only important for business continuity, but also to reduce the desensitization of building occupants to emergency alarms and evacuations.

5. Automatic sprinkler systems can often aid in the containment of fire within high fuel load compartments, but can be ineffective in multi-story atria. Simulations should be run to determine whether or not air and smoke reaching the sprinklers will be hot enough to activate the system. Typical ceiling sprinklers can be used, along with other options including long-distance throw or side wall sprinklers if required. 


\section{A.3.2.3 Structural Fire Protection}

1. A structural failure in a fire can be judged by three criteria: loss of load bearing capacity, infiltration of smoke through cracks or openings, and transfer of sufficient heat to ignite materials on the opposite side of the structural element. The latter two criteria are applicable for load-bearing fire separation wall and floor assemblies, or nonload-bearing fire separation partitions. Non-load bearing fire separation partitions and other mechanical integrity elements are treated differently than structural members and their performance must be determined through testing. These elements may impact structural performance by altering the size of the compartment if they fail and thus changing the characteristics of the fire.

2. A strategy that has been used to optimize the amount of fire protection is partial fire protection (for example, on steel structural elements), which should be validated through FEA of critical members in several design fires (Lamont et al. 2006). When proposing to leave an element unprotected, a full structural analysis must demonstrate that stability is maintained throughout the fire duration. If the analysis demonstrates a collapse (failure) mechanism, redundancy is critical. At least two separate analyses could be done to prove that (a) the element can be removed from the structure without inducing a progressive collapse of the building, or (b) the element can be allowed to thermally expand and the induced loading on the surrounding structure will not cause a progressive collapse of the building. The extent of local failure allowed is an important performance objective to be developed which may have different implications in each unique building system. Typically, elements that can be proven to remain unprotected are secondary beams and structural elements over several metres 
high from the floor. Generally, structures only supporting a roof assembly (that is not a publicly accessible roof) may not require protection, but in complex cases a design fire analysis should be done to determine whether or not fire protection for the roof structure is critical to the whole building fire safety. Additionally, frame connections are critical elements in the stability of a building and should be given specific attention in the thermal and structural analyses. Other common construction materials have seen progress internationally, such as timber with sacrificial charring layers, and concrete with optimizing rebar cover - however performance-based solutions have not yet been fully developed for these materials. The reader is encouraged to consult recent texts by Wang et al. (2013) for further advancements with materials beyond steel.

3. Another common strategy of reducing fire protection requirements is on exterior structural members. According to the building code, members at least one metre away from the façade in certain building types do not require protection (clause 3.2.2.3, NRC 2015). However, it has been justified to leave unprotected elements that are closer, through the two analysis types discussed previously.

\section{A.3.3 Examples}

There are several types of buildings that lend themselves to PBFD strategies. Large and complex buildings often have spaces that require extra care in their design to ensure occupant safety, or have been envisioned with architectural or engineering feats which would not be possible with a prescriptive code-compliant solution. There are also many buildings in which the code prescribed fire protection measures and evacuation limitations may prove overly conservative, and there are opportunities to employ fire engineering expertise to develop a more economically efficient solution for the client. Another area in 
which FSE should be implemented is in buildings with a high consequence of failure, such as hospitals, high rises, or post-emergency occupancy buildings. The following are examples of the types of projects which are considered best suited for PBFD in the opinion of the authors, as precedent projects can be used as reference. Each building type is presented along with related case studies which exemplify the methods used.

\section{A.3.3.1 Transit Stations}

Often in transit stations with terminals, concourses and waiting areas, it is desirable to have large, expansive open spaces. This desire is typically constrained by required occupant egress travel distances, so a fire engineering design and supporting analysis justifying increased distances can be beneficial. Additionally, smoke control strategies and CFD analyses can become very important and valuable in these types of spaces. Transit stations can often be divided into low-risk fire areas including the aforementioned open spaces, and high-risk fire areas including retail shops, restaurants, luggage holding and ticketing areas. The low-risk open areas often have high ceilings and may be a good opportunity to reduce the structural fire protection required. The high-risk areas can be designed for using the "cabin" or "island" strategies. For exemplary projects which have made use of these strategies, the reader is encouraged to consult case studies on the Beijing South Railway Station (Carfrae et al. 2011) and the Transbay Transit Center in San Francisco (Rini et al. 2011).

In the Beijing South Railway Station, FSE was first used to intelligently layout the facility. Areas with high fuel load such as waiting rooms were designated as fire cabins, making use of fire detection, sprinklers, and mechanical smoke exhausting, while other components of the facility were designated as islands with a low probability of fire spread 
(Carfrae et al. 2011). A CFD model of the station was developed with a range of design fires in different locations to demonstrate tenability for at least the RSET. Lastly, temperatures in the structure were calculated with the CFD model to determine fire protection requirements. Structural elements above large spaces such as the departure hall were found to have relatively low temperatures and thus require no protection, while other elements such as the columns adjacent to train platforms were given a two hour fireresistance rating.

\section{A.3.3.2 Atria}

Atria present unique fire engineering challenges within buildings due to the openness and connection of multiple stories. Smoke control can either benefit or suffer in atria depending on the geometry and height, so in each unique case comprehensive CFD should be done. Many atria are designed for their natural ventilation advantages, which can often be utilized for passive smoke exhaust systems. CFD can support the elimination of mechanical smoke exhaust systems based on larger air speeds and the size of openings at the base and top of the atrium, utilizing the natural buoyancy of hot smoke. However, if the height of an atrium becomes too high, smoke can be cooled and lose its buoyancy before it is exhausted out of the top vents, which could be detrimental to the smoke control plan. To keep the openness of atria but further control smoke flow, horizontal or vertical fire shutters may be employed to separate floors or adjacent areas in the event of a fire. Additionally, the large size of atriums usually lends itself to a partial structural fire protection strategy which could save time and money. Projects which utilized such strategies in their approval are discussed in the case studies of The Greater London Authority (GLA) Building (shown in Figure A.2) 
(O’Meagher and Ferguson 2003) and the Y2E2 Stanford University Building (Graffy et al. 2008).

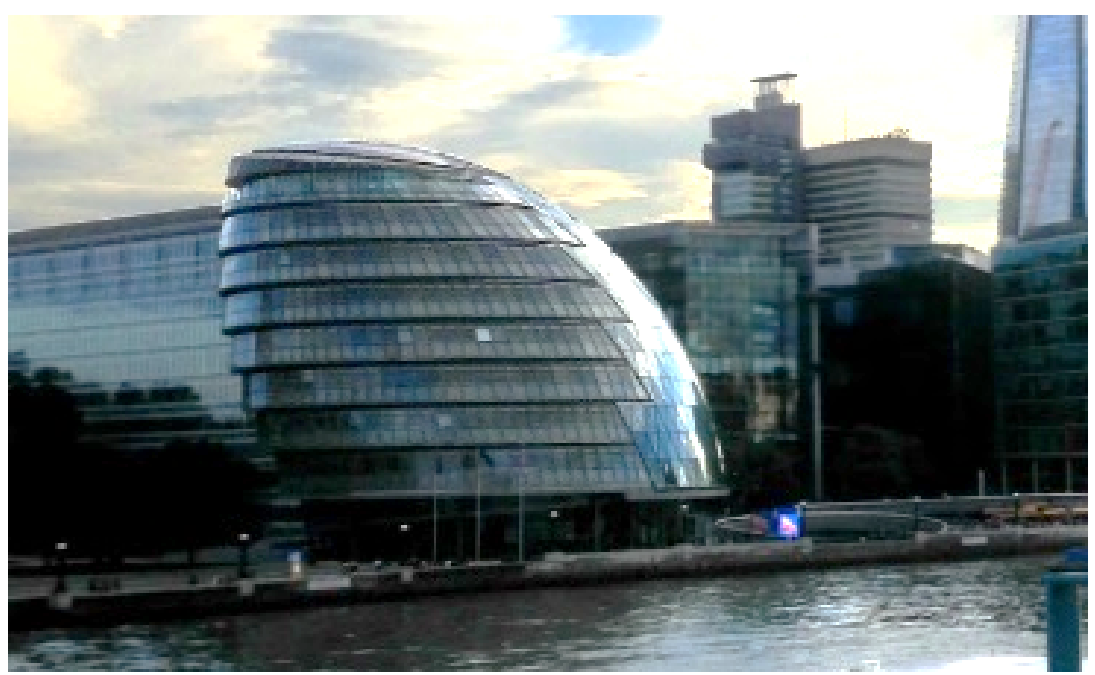

Figure A.2: The Greater London Authority Building was one of the first projects approved in London using modern FSE concepts and strategies which allowed the unique building to be executed (O'Meagher and Ferguson 2003). (Author's photo).

The GLA Building made use of automatic vents in the top of the main atrium to control smoke with automatic openings in the lower atrium either acting as an inlet for cold air or acting as an outlet for smoke in the case of a fire in the lower atrium itself ( $\mathrm{O}^{\prime}$ Meagher and Ferguson 2003). A similar approach was used for the Y2E2 building, although a key consideration here was the sheer size of the atrium. As previously mentioned, natural ventilation does not work if the atrium is too large such that smoke cools before exiting. CFD modelling was used on Y2E2 to demonstrate the smoke was sufficiently buoyant for the natural ventilation system to work (Graffy et al. 2008). Cool make-up air is provided to the atrium in the event of a fire through architectural louvres as well as automatically opening exterior doors. In addition to the CFD modelling, local authorities also required the Y2E2 building to undergo hot smoke testing during commissioning to confirm the natural ventilation system worked as intended (Graffy et al. 2008). 


\section{A.3.3.3 Stadiums and Arenas}

Sports venues are yet another type of building where extremely large open spaces are the norm, with the added challenge of thousands of spectators compressed into tightly spaced, elevated, fixed seats. Occupant egress modelling can prove very important in the design of these buildings. An analysis of pedestrian flow can be used to optimize the building layout and surrounding landscape so that movement is easy and queues are avoided. These buildings are good candidates to utilize the normal circulation areas as egress routes, as mentioned above as the third strategy within the Occupant Egress category, or horizontal evacuation plans. High-risk fire zones such as restaurants and retail shops can be treated as "cabin" zones to mitigate fire spread, and adequate smoke ventilation should be provided in case of a fire on the lower service levels. Additionally, the large open spaces and high roof create the opportunity to eliminate most of the structural fire protection required, if justified through design fire simulation and whole-building structural evaluation. The reader is directed to case studies on the First Direct Arena (Bell et al. 2014), Wembley Stadium (Dowling et al. 2015), and the Emirates Stadium (Dowling et al. 2015) in the UK for example applications of these strategies.

In the First Direct Arena, PBFD was utilized to optimize the fire protection of the roof structure, which was required to have fire protection since it provided bracing to the gravity elements of the structure (Bell et al. 2014). In the case of Wembley Stadium, CFD modelling was used to calculate temperatures in the steel roof structure and justify reduced or eliminated structural fire protection (Dowling et al. 2015). A similar approach was used for Emirates Stadium where calculated structural temperatures were compared to limiting temperatures and used to optimize the structural fire protection (Dowling et al. 2015). The 
case studies do not provide much detail in the design fires considered, details of the CFD models developed, or the extent to which structural fire engineering was used beyond simple limiting temperatures.

\section{A.3.3.4 Densely Furnished or Occupied Open Spaces}

In projects where expansive, highly-utilized spaces are desired, PBFD strategies may be required to justify less compartmentalization and longer travel distances. These spaces could include open-plan offices, conference centres, casinos and department stores, for example. In a building of these types, constructed of regular grid steel-composite structures, often the fire protection on secondary beams can be reduced or eliminated via the methodology discussed in the third case study below.

Unique occupant egress strategies (such as horizontal or even upwards evacuation - see the first two case studies below) can be developed in these situations along with a detailed design of signage and management plans, and of building geometry to promote clear, logical evacuation paths. The design of the building geometry can also be used to strategically control smoke migration throughout the space, using complex bent or curved spaces as an advantage. Often CFD and occupant modelling will be required to demonstrate that the predicted smoke behaviour and occupant RSET are justified. Additionally, in these large, open, highly-utilized spaces (unlike transit concourses with little combustible materials), large fire shutters or doors can be employed which close in the event of a fire. These systems again require a detailed evacuation strategy and method of communication to occupants as to their required egress destination. Several case studies demonstrate the use of some of the listed strategies, including the Marina Bay Sands in Singapore (Lovatt 
and Wong 2012), the Shenzhen Stock Exchange in China (Chen et al. 2014) and a UK Retail and Leisure Complex (Wang et al. 2013).

From a structural fire perspective, the UK Retail and Leisure Complex represented an opportunity to optimize the passive fire protection because of the repetition in the composite floor system. First, a series of design fires were developed using the Eurocode Parametric fire and the various ventilation configurations. A finite element model of the composite floor was developed in Vulcan (Vulcan Solutions 2016). A structural analysis of the floor at elevated temperature was performed for each design fire to determine maximum slab deflections. The stability of the floor relied on tensile membrane action, and the acceptance criteria was a deflection limit of L/20 (Wang et al. 2013). Several options for connection design were discussed including designing for the actual forces from the analysis, providing sufficient ductility, and ensuring secondary beams are supported after failure of the connections (Wang et al. 2013).

\section{A.3.3.5 Mixed-Use High Rise}

Code limitations are often very inhibiting in very tall buildings, especially when multiple functionalities are located throughout the height of the high rise. Typically the code mandates a certain number of exit stairs depending on the characteristics of the building, which could consume a large amount of profitable area in residential or commercial high rise buildings. Occupant modelling, CFD to determine ASET, and phased evacuation strategies can be used to justify a significant reduction in the required stairwells and therefore increase in lettable floor area.

On the other end of the spectrum, at times code provisions can prove insufficient for complex high-rise projects. Design fire modelling should be done to develop with 
confidence a fire protection plan which covers critical structural elements to ensure the RSET is met. Another emerging trend in tall buildings is placing the building superstructure outside of the building façade. This is an excellent opportunity to do a design fire analysis and justify the elimination of fire protection on exterior structural steel members. Examples of projects which utilized FSE strategies for the realization of iconic high-rises are found in The Shard in the UK (seen in Figure A.3) (Kitching 2012) and in the EW11 tower in the United Arab Emirates (Block et al. 2015).

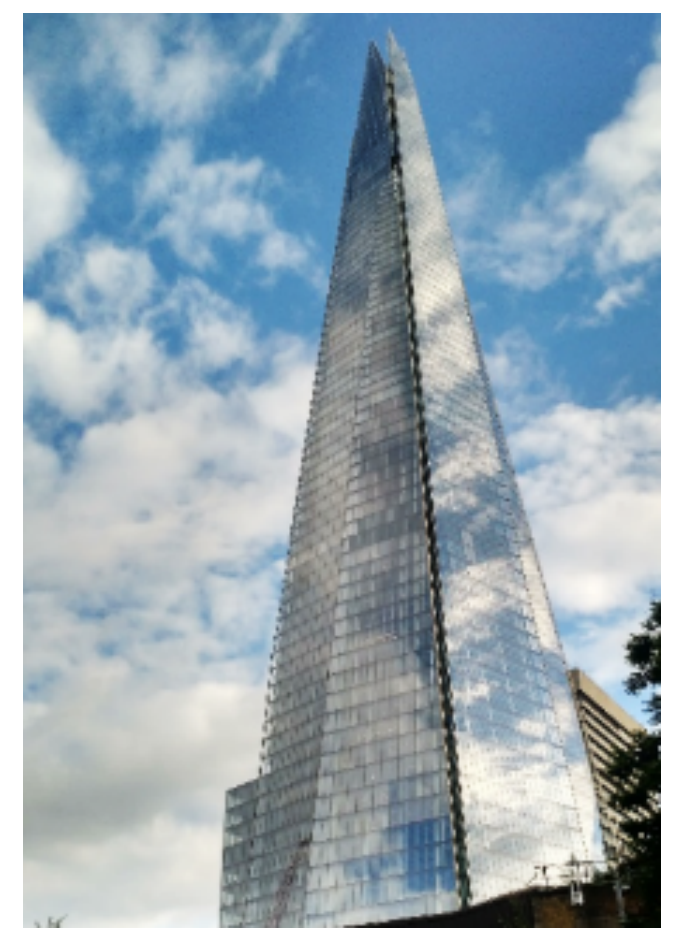

Figure A.3: The Shard high-rise building in London, England used innovative fire strategies to incorporate the functionality and spatial needs of the building (Kitching 2012). (Author's photo).

EW11 Tower utilized a unique approach where exterior structure (“diagonals") was assessed for the impact of fire exposure from interior fires. This was deemed necessary by the engineers of record given the high consequence of failure of these critical exterior elements and the unknown applicability of standard fire testing for these elements (Block 
et al. 2015). After calculating structural temperatures, the diagonals were either a) checked for their ability to support the required load at elevated temperature or b) removed from the structure and an alternate load path demonstrated.

\section{A.3.3.6 Specialized or Highly-Constrained Projects}

Highly specialized buildings or constrained projects such as historic restorations provide an opportunity for unique fire engineering strategies to be developed. In these restricted projects, often floor space is a valuable commodity due to space limitations. On a larger scale, specialized projects can involve several buildings on a site for which a total fire safety design must be completed. In this case, it is beneficial to the project to provide a fire engineering master plan to set standards for the entire campus, and to work with local authorities and fire brigades from conception to ensure a safe and approvable design. This was the case in the London Olympic Park, where evacuation modelling was used to assist with overall site evacuation as well as the bridges linking various buildings. The overall park received a fire safety master plan to ensure the various buildings and strategies were all coordinated (Taylor 2013).

Additionally in sensitive historic projects, new fire safety or protection measures must not be invasive, and must be respectful of the heritage values within the building. Often modelling can be done to either demonstrate a reduction in the required egress width (resulting in more lettable floor space) or that the inherent fire protection in the building is sufficient for the required structural integrity or ASET. In the Singapore Flyer Terminal, for example, evacuation modelling was used to demonstrate that a narrower egress width was still safe for the building resulting in an additional $400 \mathrm{~m}^{2}$ of developable floor area 
(Allsop et al. 2008) Integrating added egress routes into new additions in heritage buildings can also minimize the intervention required in historic staircases or corridors.

\section{A.4 The Future of Fire Safety Engineering in Canada}

There are potential benefits of using performance-based design in FSE in Canada which could be realized. In complex buildings with uncommon uses, layouts or geometries, FSE can improve upon a prescriptive solution by either increasing the fire protection measures to ensure that an acceptable level of safety for people and property is provided, or optimize the fire protection measures to create a more economical and efficient design. Furthermore, many new iconic building designs would be simply impossible without the use of FSE. Once these advantages of PBFD become clear to practitioners in Canada, the demand for fire safety engineers will increase and the subject being viewed as a profession will gain traction within the building engineering community. The following sections give a brief glimpse into some future and potential strategies for PBFD for use in Canada.

\section{A.4.1 Travelling Fires}

As aforementioned, the Travelling Fire methodology is a concept being developed which does not use the typical assumption that a building fire provides a uniform temperature over the whole compartment it occupies. Rather, the fire migrates throughout the space where fuel is available (see Stern-Gottfried and Rein 2012). It is still in the early stages of being accepted globally, but several of the firms mentioned in this document have utilized the Travelling Fire as a design basis for PBFD efforts (Rackauskaite et al. 2016). This type of fire simulation is thought to be exceedingly more realistic for large open spaces such as atria, transit station concourses and stadia and is currently being studied in large compartment tests by various researchers to improve user friendliness and accuracy. It 
currently considers uncertainties that exist in a real fire such as non-uniform heating of the structure, and could reduce the fire load on the structure since any single element is not typically exposed to the extreme heat for the entire fire duration. Conversely, a localized heating effect could prove to be more severe for some types of building configurations.

\section{A.4.2 Probabilistic versus Deterministic}

Once the use of design fires in Canada is well established, fire engineering could progress to a probabilistic rather than deterministic approach. Probabilistic analyses use a risk-based approach, rather than a worst-case scenario design. This analysis is based on risk factors affecting the probable frequency of a fire in a certain time period, the probability of failure due to that fire, and the consequences of that fire. The assessment is done over a large range of virtually all possible fires in all possible compartments, and a solution is reached once a predetermined degree of risk is achieved. A probabilistic approach is desirable as it considers uncertainties that invariably exist in the real world. Additionally, it is a more systematic approach and aligns with the NBCC "acceptable level of risk" methodology, which will be more comprehensible to authorities having jurisdiction and easily calibrated or compared to local building codes.

\section{A.4.3 Risk and Resilience}

Increasingly, asset owners are beginning to look at the resilience of their properties. When extreme events occur, either malicious events or natural events brought on by extreme weather and climate change, buildings must be able to sustain the events to a level that is acceptable by the users. The buildings must also be able to adapt to these events and quickly recover so that the businesses within them may operate at a necessary level. A brief consideration of the aforementioned Canadian case studies in the introduction are evident 
of these issues. A building that can remain operational during an event or shortly thereafter will enable the business within it to be more resilient. The business continuity plans of these businesses may rely on the performance of the building to be effective. PBFD offers an opportunity to quantify the risk exposure from a range of fire events and determine the expected damage levels ahead of time so that business continuity plans and repair strategies can be developed accordingly. This is a fundamental shift from the current prescriptive approach, where risk due to fire is not explicitly calculated except for extrapolating historical losses from similar building types. Further research is required internationally to better define the damage levels and required repair for various design fires, but PBFD can lead to more resilient and robust structures.

\section{A.5 Conclusions}

Once practitioners in Canada are made aware of the flexibility that PBFD can provide in innovation as seen abroad, it could be difficult to imagine a future built environment without FSE. Following the international example, FSE in Canada could become an obvious component of the integrated design process from the outset in the future. This can potentially lead to truly optimized buildings and fire protection strategies.

Should we proceed in this route, the demand for competent fire safety engineers could grow, thus increasing the number and quality of FSE education programs in Canada. This, in turn, will facilitate the advancement of design firms providing PBFD services and of authorities having jurisdiction approving these projects. This cycle, along with the active publication of FSE projects and solutions, will serve to stabilize FSE as a profession on its own like civil or mechanical engineering - creating a solid foundation upon which it will stand unique. This will lead to a future built environment in Canada where fire safety is 
demonstrated, rather than just assumed, and the public will benefit from better performing buildings that enable resilient operations.

\section{A.6 Acknowledgements}

The authors would like to acknowledge NSERC's Discovery and USRA grant programs and Entuitive.

\section{A.7 References}

Allsop, A. et al. 2008. Fire Engineering in The Singapore Flyer, The Arup Journal 2/2008, London, England, p.12.

Bailey, P. et al. 2008. Populating Virtual Buildings in The Virtual Building, The Arup Journal 2/2008, London, England, p.24.

Bartlett, R. 2005. Structural Fire Protection Determined Through Fire Protection Engineering Applications At Nova Scotia Community College, Advantage Steel, 23.

Bell, J. et al. 2014. Fire Strategy in First Direct Arena, The Arup Journal 1/2014, London, England, p.48.

Bergeron, D., Desseud, R.J., Haysom, J.C 2004. The Original and development of Canada's objective-based code concept: Proceedings of the CIB 2004 World Building Congress, Toronto, pp.1-10.

Bisby, L., Gales, J. and Maluk, C. 2013. A contemporary review of large-scale nonstandard structural fire testing. Fire Science Reviews, 2(1), pp.1-27. 
Block, F., Summers, F., Black, D. and Kho, T. S. 2015. Structural Fire Design and Approval of a 156m Tall High-End Residential Building in Abu Dhabi, CTBUH Research Paper, New York Conference, pp.678-684.

British Standards Institution. 2008. BS 9999. Code of Practice for Fire Safety in the Design, Construction and Use of Buildings, British Standards Institution, London, England, p.30.

Buchanan, A. 2002. Structural Design for Fire Safety. John Wiley \& Sons Ltd, West Sussex, England.

Buchanan, A. and Abu, A. 2017. Structural Design for Fire Safety, Second Edition, Wiley, Christchurch, NZ.

Bundy, M. et al. 2016. Structural Fire Experimental Capabilities at the NIST National Fire Research Laboratory, Fire Technology, 52, Springer, New York, USA, pp.959-966. doi:10.1007/s10694-015-0544-4

Carfrae, T. et al. 2011. Fire Engineering in Beijing South Railway Station, The Arup Journal 1/2011, London, England, pp.21-24.

Chen, J. et al. 2014. Fire Safety in Shenzhen Stock Exchange, The Arup Journal 2/2014, London, England, p.77.

Dixon, A., Ferguson, A., Lane, B. and Wardak, R. 2010. Case Studies: Beijing Olympics Water Cube in Human movement and safety: New approaches to facilities design, The Arup Journal 1/2010, London, England, p.34. 
Dowling, J., Garman, J., Pritchard, J. and Block, F. 2015. Chapter 8: Fire Engineering in Sports Stadiums in Stadium and Arena Design (2nd Edition), ICE Publishing, London, England, pp.90-93.

Gales, J., Hartin, K. and Bisby, L. 2016. Structural Fire Performance of Contemporary Post-tensioned Concrete Construction. Springer Briefs in Fire. 91pp.

Goymour, G. 2005. Passive fire protection in Plantation Place South, The Arup Journal 2/2005, London, England, p.43.

Graffy, K. et al. 2008. Fire and Smoke in Y2E2: The Jerry Yang and Akiko Yamazaki Environment and Energy Building, Stanford University, California, The Arup Journal 3/2008, London, England, p.46.

Green, M. and Joinson, J. 2010. About the Authors in The BS 9999 Handbook, British Standards Institution, London, England, p.v.

Harmathy, T. Z. 1972. A new look at compartment fires part 1. Fire Technol 8(3), pp.196217.

Harmsworth, A. and Dagenais, C. 2014. Chapter 5: Fire Safety and Protection, in Technical Guide for the Design and Construction of Tall Wood Buildings in Canada.

Her Majesty’s Stationery Office (HMSO). 1968. Behavior of structural steel in fire, proceedings of the symposium held at the fire research station, Boreham Wood, Herts, January 24th, 1967. 135pp. 
Ingberg, S. 1919. Fire Tests of Building Columns. Associated Factory Mutual Insurance Companies, The National Board of Fire Underwriters and The Bureau of Standards Department of Commerce, Chicago, Illinois, USA.

Ingberg, S. 1928. Tests of the Severity of Building Fires, NFPA Quarterly, 22, pp.43-61.

International Master of Science in Fire Safety Engineering (IMFSE). 2016. Available from: http://www.imfse.ugent.be/ [accessed 17 November 2016].

International Organization for Standardization (ISO). 2014. ISO 834-10. Fire Resistance Tests: Elements of Building Construction, International Organization for Standardization, Geneva, Switzerland.

King, D., Srikukenthiran, S. and Shalaby, A. 2014. Using Simulation to Analyze Crowd Congestion and Mitigation Measures at Canadian Subway Interchanges: Case of Bloor-Yonge Station, Toronto, Ontario Transportation Research Record Journal of the Transportation Research Board. 20pp.

Kirby, B.R., Wainman, D.E., Tomlinson, L.N., Kay, T.R. and Peacock, B.N. 1999. Natural fires in large compartments. International Journal on Engineering PerformanceBased Fire Codes 1(2), pp.43-58

Kitching, R. 2012. Steel Strategies in Case of Fire in Steel Spotlight 6, Construction News, London, England, pp.28-29.

Lamont, S., Lane, B., Flint, G. and Usmani, A. 2006. Behavior of structures in fire and real design - a case study. Journal of Fire Protection Engineering, 16(1), 5-35. 
Law, M. 1986. Translation of Research into Practice: Building Design in Fire Safety Science: Proceedings of the First International Symposium, Berkely, CA, USA, pp.603-609.

Law, M. and Beever, P. 1994. Magic Numbers and Golden Rules in Fire Safety Science: Proceedings of the Fourth International Symposium, Ottawa, ON, pp.79-84.

Lay, S. 2014. Pressurization systems do not work \& present a risk to life safety. Case Studies in Fire Safety 1, pp. 13-17.

Lennon, T. 2003. The natural fire safety concept—full scale tests at Cardington. Fire Safety Journal 38, pp.623-643. doi:10.1016/S0379-7112(03)00028-6.

Lovatt, A. and Wong, R. 2012. Fire Engineering in The Marina Bay Sands Special Issue, The Arup Journal 1/2012, London, England, pp.68-71.

National Research Council of Canada, Canadian Commission on Building and Fire Codes. 2015. National Building Code of Canada, National Research Council of Canada, Ottawa, ON.

Oasys Software. 2016a. MassMotion. Available from: http://www.oasyssoftware.com/products/engineering/massmotion.html [accessed 17 November 2016].

Oasys Software. 2016b. LS-DYNA Environment. Available from: http://www.oasyssoftware.com/dyna/en/ [accessed 17 November 2016].

O'Meagher, T. and Ferguson, A. 2003. Fire Engineering at the GLA Building, One Stop Shop in Structural Fire Engineering, University of Manchester, England. Available 
from:

http://www.mace.manchester.ac.uk/project/research/structures/strucfire/CaseStud y/steelComposite/default.htm [accessed 17 November 2016].

Ponto, M. 2006. Citadel High School: A Performance-Based Solution For Unprotected Structural Steel, Advantage Steel, 27.

Rackauskaite, E., Kotsovinos, P., Jeffers, A., and Rein., G. 2016. Structural Response of a Generic Steel Frame Exposed to Travelling Fires, 9th Structures in Fire Conference Proceedings, pp.975-982

Richardson, K. 2003. History of Fire Protection Engineering. Quincy: National Fire Protection Association.

Rini, D., Gerard, R., Almufti, I. and Nielson, G. 2011. Structural Fire Engineering for Modern Building Design - Case Study in AEI 2011: Building Integration Solutions, ASCE Proceedings of the 2011 Architectural Engineering Conference, Oakland, California, pp.351-360.

RJ Bartlett Engineering Ltd. 2014. Fire Safety Alternatives for Upper Storeys, Downtown $\begin{array}{llll}\text { St. Johns, } & \text { NL. }\end{array}$ http://www.stjohns.ca/sites/default/files/files/publication/FIRE\%20SAFETY\%20 ALTERNATIVES\%20REPORT.pdf [accessed 17 November 2016].

Society of Fire Protection Engineers (SFPE). 2000. SFPE Engineering Guide to Performance-Based Fire Protection Analysis and Design of Buildings, Society of Fire Protection Engineers. 
Stern-Gottfried, J. and Rein, G. 2012. Travelling Fires for Structural Design Part I: Literature Review in Fire Safety Journal 54, pp.74-85. doi:10.1016/j.firesaf.2012.06.003

Stern-Gottfried, J., Rein, G., Bisby, L. and Torero, J. L. 2010. Experimental review of the homogeneous temperature assumption in post-flashover compartment fires. Fire Safety Journal 45, pp.249-261. doi:10.1016/j.firesaf.2010.03.007.

Taylor, A. 2013. Site-Wide Perspective in Fire Risk Management Journal July/August 2013, Fire Protection Association, Gloucestershire, England, pp. 38-41.

The Steel Construction Institute (SCI). 1991. Structural Fire Engineering: Investigation of Broadgate Phase 8 Fire. The Steel Construction Institute Publication 113, Berkshire, England, 88pp.

Torero, J. L. 2012. Fire Safety Engineering: Profession, Occupation or Trade? in International Fire Professional 1(1), Institution of Fire Engineers, England, pp.1822.

Torero, J. L., Majdalani, A. H., Abecassis-Empis, C. and Cowlard, A. 2014. Revisiting the Compartment Fire. Fire Safety Science 11, pp.28-45. 10.3801/IAFSS.FSS.11-28.

Trenton Fire. 2016. Company Profile and Capability Statement, Trenton Fire, London, England. Available from: https://www.trentonfire.co.uk/pdf/Trenton-Fire-Profileand-Capability-Statement.pdf [accessed 17 November 2016].

ULC. 2014. CAN/ULC-S101-14 Standard Methods of Fire Endurance Tests of Building Construction and Materials, Ottawa. 
University of Liège. 2016. SAFIR, School of Engineering and Computer Science. Available from: http://www.facsa.ulg.ac.be/cms/c_1584029/en/safir [accessed 17 November 2016].

Usami, A. et al. 2000. Behaviour of steel framed structures under fire conditions: Main Report, PIT Project, University of Edinburgh, 76pp.

Vulcan Solutions. 2016. Vulcan. Available from: http://www.vulcan-solutions.com/ [accessed 17 November 2016].

Wang, Y., Burgess, I., Wald, F. and Gillie, M. 2013. Performance-Based Fire Engineering of Structures, CRC Press, Boca Raton, FL, USA.

Woodrow, M., Bisby, L. and Torero, J. 2013. A nascent educational framework for fire safety engineering. Fire Safety Journal, 58, pp.180-194. 


\section{Appendix B - Finite Element Modelling of Post-}

\section{Tensioned Timber Beams under Fire Conditions}

\section{B.1 Abstract}

In order to implement innovative timber structures globally, performance-based design using numerical models are often required. A model was developed to simulate posttensioned (PT) timber beams in FEM software Abaqus and compared to three furnace beam experiments. A sequentially coupled heat transfer - mechanical analysis was developed and validated. The timber was treated as orthotropic both in elasticity and plasticity, and was modelled with temperature-dependent properties. The simulation results showed good agreement with both the thermal results and the mechanical analysis. The thermal results matched the thermocouple readings well and the char depths were predicted to within 4 $\mathrm{mm}$. The failure modes and times were accurately predicted (within 5\% accuracy), and the deflection behaviour was reasonably predicted compared to the experimental data.

\section{B.2 Introduction and Background}

Along with the innovation of new building systems, the ways in which we design them must also advance in order for the systems to be implemented by practitioners. This is especially true for the fire engineering design of novel construction methods as their performance under fire is investigated. In order to complete a performance-based design of such systems, a computer model is often required to demonstrate system behaviour. Predictive models are often valuable in the design and optimization of such complex structures providing they can be validated against meaningful data. 
Post-tensioned (PT) timber is a relatively nascent building system in which the structure is composed of engineered timber elements which are pre-stressed with high-strength steel tendons. This study has focused on PT timber beam elements. The engineered timber may take the form of Laminated Veneer Lumber (LVL) or Glulam, for example, as a structural element which has a central cavity running along its length. The steel tendons are typically placed eccentrically below the neutral axis of the beams, in order to induce a negative moment and overall compression in the beam as it is pre-stressed. Analogous to the widelyused pre-stressed concrete system, such an approach allows for longer span beams using smaller cross-sections. A typical PT timber beam schematic is shown in Figure B.1.

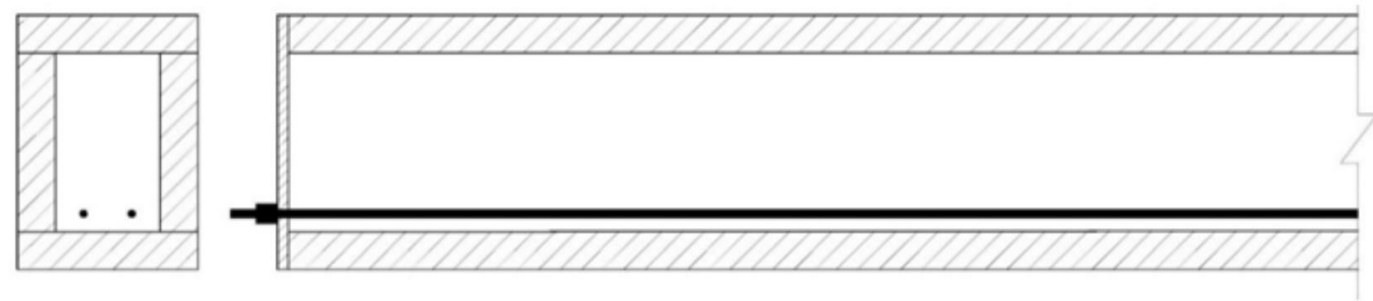

Figure B.1: Schematic of typical PT timber beam cross-section showing built up wood webs and flanges and high-strength steel tendons, along with section cut through beam length showing tendon anchorage with a steel plate at the end

The PT timber system was developed as part of a research study at the University of Canterbury, and was originally studied for its enhanced seismic performance ${ }^{1}$. The system has been used in buildings internationally and has been proposed for several planned buildings in North America. There have been a number of large-scale experiments done on the system at ambient temperature, and only two experimental studies done in fire conditions. These studies were done by Spellman in $2012^{2}$ and Costello in $2013^{3}$. Spellman completed three beam furnace tests, of which one was reasoned to be an anomaly because of premature failure due to possible manufacturing imperfections. Later, Costello 
completed a single furnace test along with several ambient beam tests in order to further investigate the failure mechanisms observed. The two experiments from Spellman's research that were not abnormal (Beams A and C) and Costello's furnace test were all modelled and are discussed herein.

In order to capture the complex behaviour of a PT timber beam, a three-dimensional Finite Element Model (FEM) is required. The commercially available general purpose FEM software package, Abaqus was chosen to build the model as it has a wide variety of material models available, and has the ability to simulate both heat transfer and mechanical analyses. Abaqus is a valuable research tool which may be used to demonstrate the ability to model structural systems, after which a refined, simpler and less costly model may be developed for use by practitioners. The following section will detail the creation of the model, while the results section discusses the outcomes from each simulation compared to their respective experimental data.

\section{B.3 Model Objectives and Setup}

The primary objective of the model is to provide a setup within a widely-used FEM software that may be used to predict the behaviour of a PT timber beam in fire conditions reasonably accurately. As many experiments were also done under ambient conditions, and beams will often have to be designed for both ambient and fire conditions, it was desirable for the model to provide accurate results in both types of analyses. Additionally, it was desired that the model should be applicable for structures using both LVL and Glulam timber, as the preference for the type of engineered timber varies internationally and both products have been proposed for use with the system. Thus, the material model was simplified in such a way as to ensure this suitability. 


\section{B.3.1 Material Models}

The timber material's mechanical properties were treated as orthotropic with transverse isotropy in order to be suitable for modelling LVL or Glulam timber. The stiffness reductions suggested in Eurocode 5 Part 1-2 Annex B ${ }^{4}$ were used. They are given as functions of temperature and of stress state, varying from tension to compression, as shown in
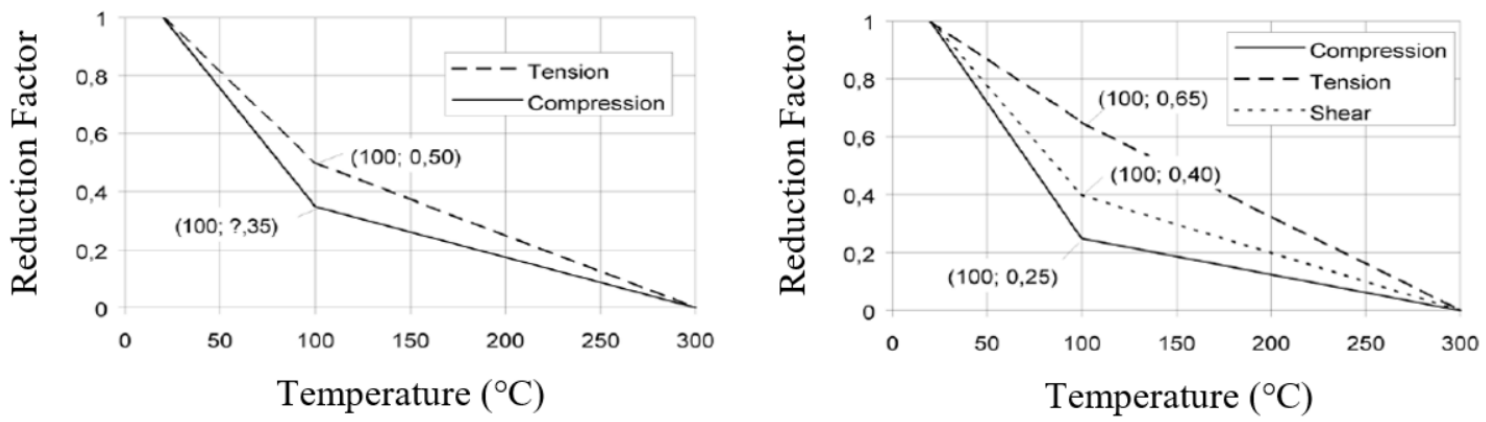

Figure B.2.
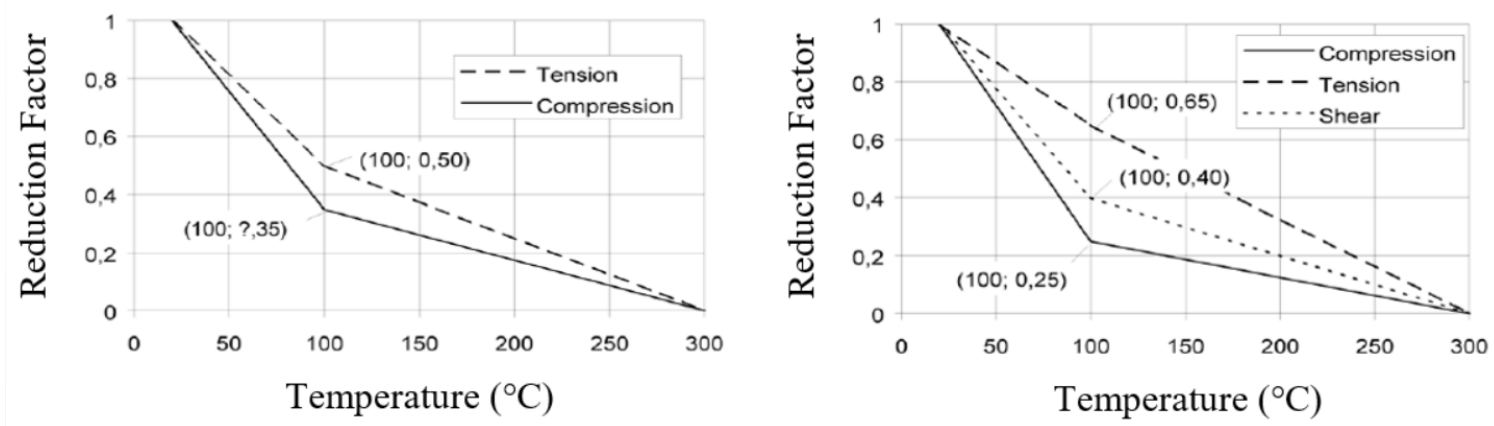

Figure B.2: Eurocode 5 Part 1-2 Annex B suggested reduction factors for elastic modulus (left) and strengths (right) ${ }^{4}$

For the Elastic material properties, an Engineering Constants model was used. This allows the definition of elastic moduli in each orthogonal direction as a function of temperature, a summary of which is given in Table B.1. The manufacturer specified an elastic modulus of $13200 \mathrm{MPa}$ and a shear modulus of $660 \mathrm{MPa}$ for the specimens of both Spellman ${ }^{2}$ and 
Costello ${ }^{3}$. The values used for the elastic moduli in the transverse directions, Poisson's ratios, and the rolling shear modulus were determined based on extensive material testing done by Van Beerschoten ${ }^{5}$.

The Engineering Constants material elasticity definition does not distinguish between moduli for tension and compression, so only one value could be used to represent both. This only affected the definition of the moduli at $100^{\circ} \mathrm{C}$, where the reduction factor differs for tension and compression. The reduction factor of 0.5 (for tension) ${ }^{4}$ was used as the compression zone of the beam is largely unaffected by temperature, due to the top flange being insulated from the heat exposure. Additionally, the modification factors are given only for the principal elastic modulus. Thus, it was assumed that the reduction factors could also be used for the other two material directions and all the shear moduli. Abaqus specifies that the minimum parameter reduction allowed is $1 / 100$ th of the initial magnitude, so this requirement was used instead of the Eurocode specification of zero ${ }^{4}$.

Table B.1: Input parameters for engineering constants orthotropic elasticity as a function of temperature

\begin{tabular}{|l|l|l|l|l|l|l|l|l|l|}
\hline $\begin{array}{c}\mathbf{E}_{\mathbf{1}} \\
(\mathbf{M P a})\end{array}$ & $\begin{array}{c}\mathbf{E}_{\mathbf{2}} \\
(\mathbf{M P a})\end{array}$ & $\begin{array}{c}\mathbf{E}_{\mathbf{3}} \\
\mathbf{( M P a )}\end{array}$ & $\mathbf{v}_{12}$ & $\boldsymbol{v}_{13}$ & $\mathbf{v}_{\mathbf{2 3}}$ & $\begin{array}{c}\mathbf{G}_{\mathbf{1 2}} \\
(\mathbf{M P a})\end{array}$ & $\begin{array}{c}\mathbf{G}_{13} \\
(\mathbf{M P a})\end{array}$ & $\begin{array}{c}\mathbf{G}_{\mathbf{2 3}} \\
(\mathbf{M P a})\end{array}$ & $\begin{array}{c}\text { Temperature } \\
\left({ }^{\circ} \mathbf{C}\right)\end{array}$ \\
\hline 13200 & 400 & 400 & 0.55 & 0.55 & 0.2 & 660 & 660 & 100 & 0 \\
13200 & 400 & 400 & 0.55 & 0.55 & 0.2 & 660 & 660 & 100 & 20 \\
6600 & 200 & 200 & 0.55 & 0.55 & 0.2 & 330 & 330 & 50 & 100 \\
132 & 4 & 4 & 0.55 & 0.55 & 0.2 & 6.6 & 6.6 & 1 & 300 \\
\hline
\end{tabular}

In order to model the complex plasticity and failure behaviour of wood, several material models were considered within Abaqus. Ideally, the material model should define failure strengths in each material direction which may be defined as functions of both temperature and stress state. As no such material model exists within Abaqus, and the desire was to not use a user subroutine to maintain convenience for practitioners, it was determined that the 
most suitable model available was the Hill's Potential Function within the Plastic material definition. Similarly to the Engineering Constants model, this definition does not distinguish between tensile and compressive strengths.

The manufacturer of the LVL for Spellman's tests gave a compressive strength parallel to grain of $45 \mathrm{MPa}$ and for Costello's test of $38 \mathrm{MPa}$, while both manufacturers specify a tensile strength parallel to grain of $33 \mathrm{MPa}{ }^{2,3}$. A purely elastic analysis showed that the material stresses parallel to grain always remained below $33 \mathrm{MPa}$, therefore $33 \mathrm{MPa}$ was used as the yield strength. This was also used in conjunction with the tensile strength reduction factor of $0.65^{4}$, as the compressive zone remained largely at ambient temperature (due to three-sided exposure). The tensile stresses perpendicular to grain in the analyses were negligible, therefore the strength for compression perpendicular to grain was used in the transverse directions, along with the corresponding reduction factor of $0.25^{4}$. The manufacturer shear strength of 5.3 $\mathrm{MPa}$ was used in all shear directions with a reduction factor of 0.4 for longitudinal shear and 0.25 for rolling shear ${ }^{2,3,4}$.

The Plastic material properties in Abaqus was defined simply as a baseline yield stress, $\sigma_{0}$, as a function of temperature, as is shown in Table B.2. The Hill's Potential Function then calculates a corresponding baseline shear strength, $\tau_{0}=\sigma_{0} / \sqrt{3}$. The material strengths in each direction are then defined as discussed above (summarized in Table B.2), using a ratio between that stress and the baseline yield or shear strength, respectively. For example, the ratio of the longitudinal strength, $R_{11}=f_{11} / \sigma_{0}$ and the ratio of the first longitudinal shear strength, $R_{12}=f_{12} / \tau_{0}$. The ratios are summarized in Table B.3 as they are input into Abaqus. 
Table B.2: Strength values used for calculation of the Hill's Potential Function ratios

\begin{tabular}{|l|l|l|l|l|l|l|l|l|}
\hline $\begin{array}{c}\boldsymbol{\sigma}_{\mathbf{0}} \\
(\mathbf{M P a})\end{array}$ & $\begin{array}{c}\boldsymbol{f}_{\mathbf{1 1}} \\
(\mathbf{M P a})\end{array}$ & $\begin{array}{c}\boldsymbol{f}_{\mathbf{2 2}} \\
(\mathbf{M P a})\end{array}$ & $\begin{array}{c}\boldsymbol{f}_{\mathbf{3 3}} \\
(\mathbf{M P a})\end{array}$ & $\begin{array}{c}\boldsymbol{\tau}_{\mathbf{0}} \\
(\mathbf{M P a})\end{array}$ & $\begin{array}{c}\boldsymbol{f}_{\mathbf{1 2}} \\
(\mathbf{M P a})\end{array}$ & $\begin{array}{c}\boldsymbol{f}_{\mathbf{1 3}} \\
(\mathbf{M P a})\end{array}$ & $\begin{array}{c}\boldsymbol{f}_{\mathbf{2 3}} \\
(\mathbf{M P a})\end{array}$ & $\begin{array}{c}\text { Temperature } \\
\left({ }^{\circ} \mathbf{C}\right)\end{array}$ \\
\hline 33 & 33 & 10 & 10 & 19.1 & 5.3 & 5.3 & 5.3 & 0 \\
33 & 33 & 10 & 10 & 19.1 & 5.3 & 5.3 & 5.3 & 20 \\
21.45 & 21.45 & 2.5 & 2.5 & 12.4 & 2.12 & 2.12 & 1.325 & 100 \\
0.33 & 0.33 & 0.1 & 0.1 & 0.191 & 0.053 & 0.053 & 0.053 & 300 \\
\hline
\end{tabular}

Table B.3: Input parameters for strength ratios used in Hill's Potential Function for plasticity

\begin{tabular}{|l|c|c|c|c|c|l|}
\hline $\boldsymbol{R}_{\mathbf{1 1}}$ & $\boldsymbol{R}_{\mathbf{2 2}}$ & $\boldsymbol{R}_{\mathbf{3 3}}$ & $\boldsymbol{R}_{\mathbf{1 2}}$ & $\boldsymbol{R}_{\mathbf{1 3}}$ & $\boldsymbol{R}_{\mathbf{2 3}}$ & $\begin{array}{l}\text { Temperature } \\
\left({ }^{\circ} \mathbf{C}\right)\end{array}$ \\
\hline 1 & 0.303 & 0.303 & 0.278 & 0.278 & 0.278 & 0 \\
1 & 0.303 & 0.303 & 0.278 & 0.278 & 0.278 & 20 \\
1 & 0.117 & 0.117 & 0.171 & 0.171 & 0.107 & 100 \\
1 & 0.303 & 0.303 & 0.278 & 0.278 & 0.278 & 300 \\
\hline
\end{tabular}

The thermal properties were treated as isotropic corresponding to heat transfer perpendicular to grain, as very little heat transfer along the grain of the wood was expected due to uniform heating. A wet density of $530 \mathrm{~kg} / \mathrm{m}^{3}$ was assumed for the Radiata pine used in the experiments, and the moisture content (MC) was assumed to vary from $6 \%$ to $12 \%$, the former having been measured in wood acclimatized to a lab setting and the latter being a typical value for wood structures. This variance was included as the experimentalists did not report the moisture content of the wood in their theses, and the former matched the thermal results of Spellman well while the latter provided more accurate results for Costello's tests. The latent heat of evaporation of water was included in the thermal model as $271.2 \mathrm{~kJ} / \mathrm{kg}_{\text {wood }}$ for $12 \% \mathrm{MC}$ and $135.6 \mathrm{~kJ} / \mathrm{kg}_{\text {wood }}$ for $6 \% \mathrm{MC}$, based on a heat of evaporation for water of $2260 \mathrm{~kJ} / \mathrm{kg}_{\text {water }}$. The density, conductivity, and specific heat of wood were all defined following the recommendations from Eurocode 5 Part 1-2 Annex B 4. The jump in the effective specific heat definition was excluded as the evaporation of water was accounted for using the latent heat method, as this provided more stable results. 
There were two types of steel used in the experiments. The first was regular strength steel for the loading, support and anchorage plates, and the second was high-strength steel used for the PT tendons. In the experiments and in the simulations, all steel parts remained well within their elastic range and well below $100^{\circ} \mathrm{C}$, while degradation in steel tendons is not expected below $200^{\circ} \mathrm{C}$. Therefore, a simple isotropic elasticity model was defined with an elastic modulus of $200 \mathrm{GPa}$ and a Poisson's ratio of 0.3 .

\section{B.3.2 Analysis Setup}

The full simulation was done as a sequentially coupled heat transfer - mechanical analysis. First, the heat transfer analysis was done on just the three-dimensional beam part with heat transfer brick elements. The beam was exposed to the fire on three sides with a convection interaction (using a film coefficient of $25 \mathrm{~W} / \mathrm{m}^{2} \mathrm{~K}$ ) and with a radiation interaction (using an emissivity of 0.8 ). The furnace temperature was defined as an amplitude in Abaqus and used as the sink/ambient temperature for both interactions. The internal cavity surfaces were defined with a cavity radiation approximation where the average surface temperature at each step was used as the ambient temperature for heat transfer. The thermal results were then compared with the available experimental data and used subsequently in the mechanical analysis.

In the succeeding mechanical analysis, the timber beams were modelled in Abaqus using three-dimensional linear, reduced-integration stress brick elements. The beams in Spellman's two tests were simply supported as was indicated in his thesis (Costello 2013). In Costello's furnace test, the beam rested on the concrete frame, creating a more fixed support as it did not allow for much rotation ${ }^{3}$. The boundary conditions were applied 
through a hard contact interaction between the beam and steel support plates, which had the corresponding degrees of freedom restrained at a coupled reference point.

The PT tendons were modelled as wire elements tied to anchorage plates at each end of the beams, which were then tied to the end faces of the beams. In the first step (after initial), the PT load was applied as a bolt load on a central partition of the tendon which uses a change in length to apply the prescribed load. The change in length was then fixed for all subsequent steps. The vertical load plates were tied to the top of the beams in a four-point loading scheme with the total load applied as a pressure over the plate top surfaces in the second step, and was held constant over the subsequent step. Following the PT and vertical loading steps, the fire exposure step was created. This was input as a predefined field using the output database file created from the heat transfer analysis using incompatible meshes. This allowed the beam mesh to be optimized for each type of analysis. The numerical results were compared to the available time-deflection data from the experimental results.

\section{B.4 Results}

There are two sets of results to be discussed. The first are the thermal results compared to the experimental thermocouple readings, and the second are the mechanical results compared to the experimental recorded deflections. Select results are shown herein to demonstrate the model outcomes.

\section{B.4.1 Heat Transfer Analysis Results}

The thermal results from the numerical model of Spellman's tests were measured at the same locations as in the experiments, as shown in Figure B.3, with $0 \mathrm{~mm}$ representing the exposed surface. 


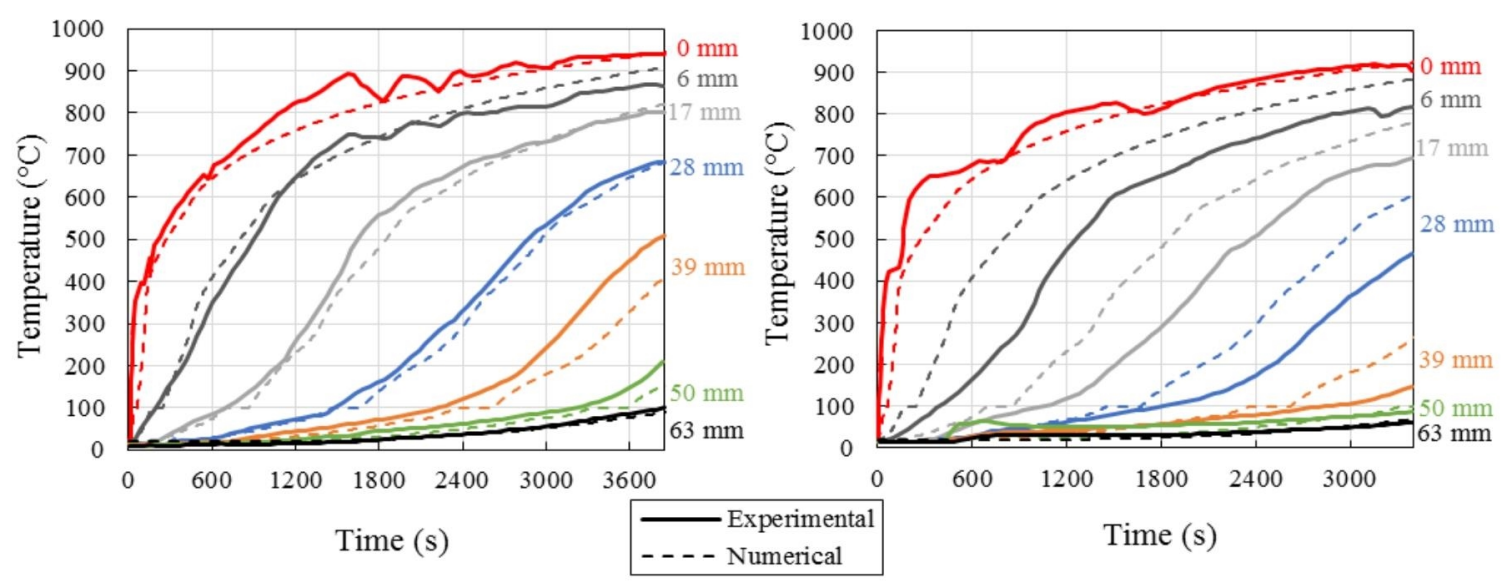

Figure B.3: Comparison between experimental ${ }^{2}$ and numerical thermal results through the web thickness for Spellman's Beam A (left) and Beam C (right)

The thermal results from the simulation of Spellman's Beam A matched very well with the experimental data, as can be seen in the left plot in Figure B.3. The char depth predicted was $44 \mathrm{~mm}$ compared to the experiment reported char depth of $47.5 \mathrm{~mm}$. The temperature results for the numerical model of Beam $\mathrm{C}$ were notably higher than the thermocouple readings, but did seem to follow a similar trend. Spellman noted in his thesis that the thermocouple readings for Beam $\mathrm{C}$ were substantially lower than those measured in the other furnace tests. Upon inspection of the final char depth in the model for Beam C (taken as the $300^{\circ} \mathrm{C}$ isotherm), the model predicts a depth of $39 \mathrm{~mm}$, while the char depth reported was $40 \mathrm{~mm}^{2}$, showing good agreement with the thermal results in this respect.

\section{B.4.2 Mechanical Analysis Results}

The numerical deflections were extracted at the mid-span for Costello's test and were the average of the load plate deflections in Spellman's tests to match the data acquisition method of each ${ }^{2,3}$. A summary of the failure times is given in Table B.4, and a comparison between the numerical and experimental results for all three tests discussed is shown in Figure B.4. 
Table B.4: Comparison of beam experiment ${ }^{2,3}$ and model failure times

\begin{tabular}{|l|l|l|l|}
\hline Beam Test & $\begin{array}{c}\text { Experiment Failure Time } \\
\text { (min) }\end{array}$ & $\begin{array}{c}\text { Model Failure Time } \\
\text { (min) }\end{array}$ & \multicolumn{1}{|c|}{$\begin{array}{c}\text { Difference } \\
\text { (min) }\end{array}$} \\
\hline Spellman A & 64 & 66 & +2 \\
Spellman C & 56 & 53 & -3 \\
Costello & 29 & 29 & 0 \\
\hline
\end{tabular}

In general, the simulation results showed good agreement with both the failure time and modes predicted, and reasonably predicted the deflection behaviour. All simulations stopped due to excessive deformations at the failure point, where runaway deflections are seen in the results. Costello's beam test was the most accurately predicted including the failure mode, images of which are shown in Figure B.5. In Spellman's tests, the predicted behaviours slightly differed from the experiments, particularly the failure in Beam A which was much more abrupt than the experiment. The differences could be due to the lack of cracking in the numerical model. In the experiment, large cracks propagated through the beam which would have resulted in a more gradual failure, while the model beam remained intact for the entire analysis causing it to have a higher stiffness than observed in the real deflection behaviour.

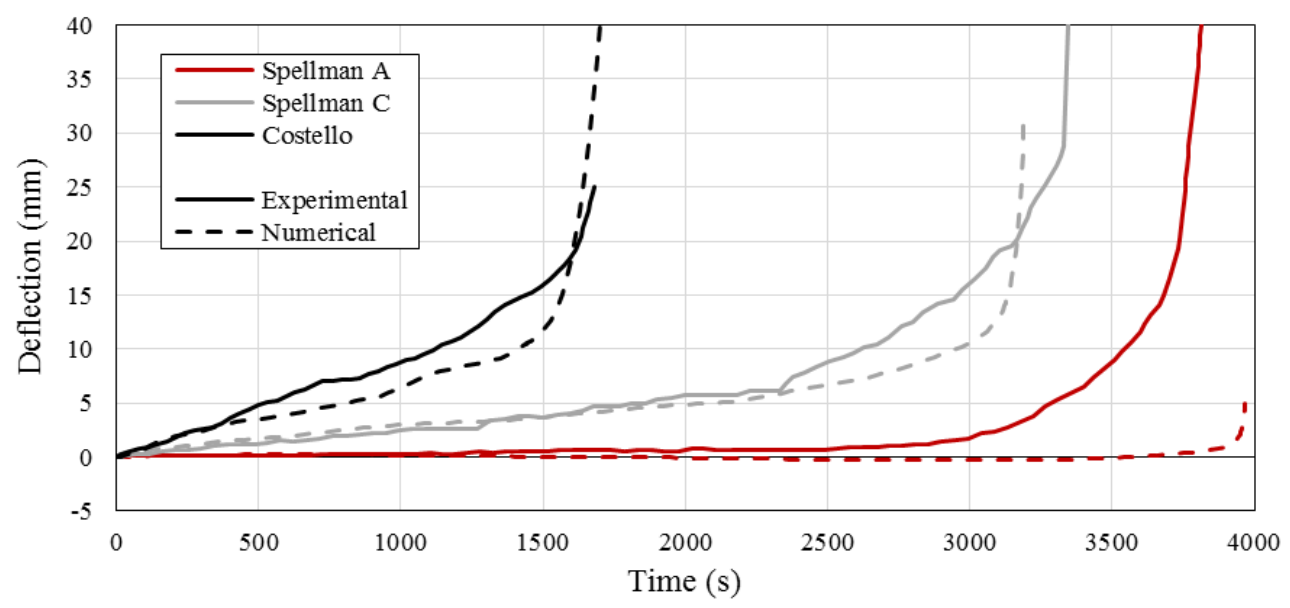

Figure B.4: Comparison between experimental ${ }^{2,3}$ and numerical deflection results 

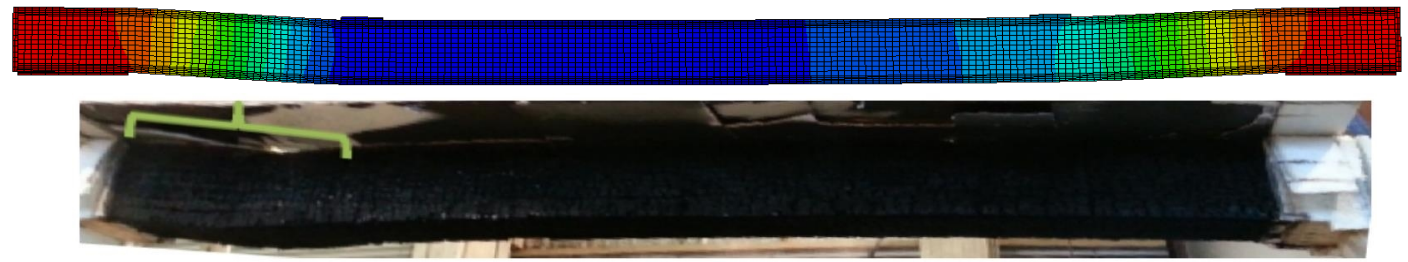

Figure B.5: Failure region in left side shear zone in simulation results and experiment (modified from ${ }^{3}$ )

\section{B.5 Recommendations and Conclusions}

Overall, the approximation of the PT timber beam behaviour in fire conditions using the Hill's Potential Function for plasticity in Abaqus was reasonably accurate, predicting failure times within 5\% accuracy. The model could be improved with a material model which allows for both material directionality and stress state dependence. Additionally, with the implementation of a crack propagation model, the exact failure mechanisms observed in the experiments could be captured. In conclusion, the model presented promising results and could be refined in a designated model to be made available for use by practitioners for performance-based design.

\section{B.6 References}

1. Palermo, Alessandro, et al., "Seismic design of multi-storey buildings using Laminated Veneer Lumber (LVL)", Proceedings of the New Zealand Society for Earthquake Engineering Conference, Paper 14, 11-13 March, 2005.

2. Spellman, Phillip, "The Fire Performance of Post-Tensioned Timber Beams”, Master's Thesis, University of Canterbury, 2012.

3. Costello, Reuben, "The Fire Performance of Post-Tensioned Timber Buildings", Master's Thesis, University of Canterbury, 2013.

4. "Part 1-2: General - Structural Fire Design", Eurocode 5: Design of Timber Structures, European Committee for Standardization, 2004, pp.48-51.

5. Van Beerschoten, Wouter, "Structural Performance of Post-tensioned Timber Frames under Gravity Loading”, PhD Thesis, University of Canterbury, 2013. 


\section{Appendix C - Performance of Adhesives in Glulam after}

\section{Short Term Fire Exposure}

\section{C.1 Abstract}

As engineered timber, such as Glulam, is seeing increasing use in the creation of tall timber buildings, building codes are adapting to allow for this. In order for this material to be used confidently and safely in one of these applications, there is a need to understand the effects that fire can have on an engineered timber structural member. The post-fire resilience aspect of glulam is studied herein. Two sets of experiments are performed to consider the validity of zero strength guidance with respect to short duration fire exposure on thin glulam members. Small scale samples were heated in a cone calorimeter to different fire severities. These samples illustrated significant strength loss but high variability despite controlled quantification of char layers. Large scale samples were heated locally using a controlled fuel fire in shear and moment locations along the length of the beam respectively. Additionally, reduced cross section samples were created by mechanically carving a way an area of cross section equal to the area lost to char on the heated beams. All of the samples were then loaded to failure in four-point (laterally restrained) bending tests. The beams that have been burnt in the shear region were observed as having a reduction in strength of up to $34.5 \%$ from the control beams. These test samples displayed relatively little variability, apart from beams that displayed material defects. The suite of testing indicated that zero strength guidance may be under conservative and may require increasing from $7 \mathrm{~mm}$ up to as much as $23 \mathrm{~mm}$. 


\section{C.2 Introduction}

Engineered timber products are structural materials characterized by the union of wood laminae with polymer-based adhesives. Common uses of engineered timber consistently employed in structural design are built-up sections which provide stability, as well as insulation and integrity. Common examples include: Glulam columns and beams and Cross Laminated Timber (CLT) walls and floors. Canadian practitioner interest in engineered timber has been encouraged by a recent efforts to advocate 12 story tall timber construction the National Building Code of Canada (NBCC) for 2020. The code also uses language describing the use of heavy timber construction which is congruent with engineered timber products. The expansion of CSA O86 timber guidance for engineered timber construction has followed. As demonstration projects, some practitioners have begun to build these high-rise timber structures in Canada. However, with the pending code changes, there remains peaked interest from various Canadian practitioners in methods and sufficient background knowledge to design these buildings with confidence. One of the most notable demonstration projects in Vancouver, Canada is as Brock Commons. It is an exemplar engineered timber structure as it exceeds height limitations using a regional building code. In this building nearly all Glulam and CLT elements were encapsulated with multiple layers of fire rated gypsum board to simplify the fire design (Jeanneret et al., 2017). This procedure allowed the building to meet required fire resistance rating using the additive rules. To the knowledge of the authors, there was no attempt to quantify the degradation of the timber itself as contributing to part of the fire resistance calculation beneath the gypsum board. The fire rating of Brock Commons may be thought as significantly higher once the resistance of the timber itself is considered. The validity of this assessment is multi-faceted 
and indeed in need of additional research. Following Brock Commons, an exhaustive study of engineered timber compartments using encapsulated CLT has been made publically available by researchers at NIST and NRC (Su et al., 2018). In that study it was identified that a complicated breakdown of adhesives in engineered timber were occurring at high temperatures leading to delamination as well as unique and complicated compartment fire dynamics for exposed timber. Those researchers have advocated that engineered timber adhesives receive more research attention.

When exposed to a fire, timber begins to enter pyrolysis and forms a char layer on the exposed surfaces. In the case of engineered timber, other effects such as adhesive degradation also begin to occur and introduce more complex failure mechanisms (see Quiquero et al., 2016). Current guidance allows for a remaining cross-section of the fireexposed member to be considered "undamaged" below the char and pyrolysis layers. This is typically done by assuming a zero-strength layer beyond the char front, which is meant to implicitly account for degradation effects that include the break-down of adhesive, loss of moisture and pyrolysis of wood. Therefore, guidance does exist to permit unencapsulated timber from a stability perspective. However, this guidance is in need of review as the current quantification of the zero-strength layer has been debated in recent literature, ranging from 7 to $16 \mathrm{~mm}$ depending on the type of fire and the severity (Lange et al., 2015; and Gales et al., 2018). The ability to expose a building's engineered timber is architecturally desirable and therefore studying adhesive breakdown, given documented uncertainty, is of use and indeed a novel contribution. In order for engineered timber to be defensibly left exposed, a greater understanding of the underlying thermal-mechanical properties is required. The study herein addresses this need. To focus the study, the authors 
predominately considered the after-fire performance of Glulam adhesive as the subject of investigation. The role of Glulam's as the primary stability mechanism of most tall timber structures renders intimate knowledge of its behaviour highly important. CLT and other engineered timber products rely on similar adhesives, so some conclusions herein may extend to such other products. The post-fire performance gives indication to the degree of reparability after a fire, which helps provide confidence to authorities having jurisdiction in approving this construction type. Future work is to follow that will specifically consider the in-fire performance. While this study explicitly considers Canadian design context, as the materials tested are provided by local manufacturers, its results are generally applicable to other jurisdictions that are also developing tall timber guidance. The research herein should not be construed as an attempt to establish fire resistance metrics or comparators to ISO / ASTM standard fire exposures. The study herein is attempting to consider the fire resilience of glulam by studying the underlying degradation breakdown of complicated engineered adhesive polymers in two equally important test phases. Phase 1 and 2 deal with explicit and conservative approximation of the zero strength layer at two different scales. Additionally, the small scale experiments focus directly on the specific adhesive performance while the larger scale tests introduce effects such as combined loading and material defects which may influence the failure of the specimens. Next steps that are required for renewed guidance for tall timber structures is provided.

\section{C.3 Background on Adhesive Degradation}

The behaviour of wood in fire is well known and highly predictable based on decades of experiments on the subject. However, the introduction of adhesive introduces highly complex mechanisms that are still in need of study. In particular, the way the adhesive 
interacts with the char layer and the adhesive degradation itself has scarcely been investigated in realistic building fire configurations.

\section{C.3.1 Charring Behaviour of Engineered Timber}

Engineered timber products are largely believed to char in much the same way that solid timber would. Under extreme heating, wood begins to undergo a process known as pyrolysis around $100^{\circ} \mathrm{C}$ as its chemical properties begin to change. The wood strength begins to degrade until $300^{\circ} \mathrm{C}$ which is recognised as the charring temperature of wood, at which point the pyrolysis process has completed and the wood is completely converted to char. After this point, char may continue to develop as the wood behind the char layer is heated, and the exposed char continues to oxidize until it begins to crack and flake off, or is fully consumed. Although the charring rate is realistically a transient property depending on the degree of char that has already formed and the amount of heat exposure, standardized constant charring rates have been developed. For example, a commonly quoted value for Spruce-Pine-Fir (SPF) Glulam is $0.7 \mathrm{~mm} / \mathrm{min}$. This rate is meant to encompass the initial rapid char phase while fresh wood builds up an insulating layer of char which then slows the charring rate to a much lower value. However, it has also been derived from standard fire exposure so questions of relevance have been raised. Modified charring rates for Eurocode parametric fires have been proposed (Hopkin, 2016). The codes typically prescribe an additional zero-strength layer of $7 \mathrm{~mm}$ beyond the char depth that accounts for the loss of strength in the pyrolysis zone of the heated timber. An in-depth study was done by Lange et al. (2015) in which numerous engineered timber beams were loaded in furnace tests exposed to standard and parametric fires. That research suggested that this layer may range from $8 \mathrm{~mm}$ for a short, intense fire, up to $16 \mathrm{~mm}$ for a longer fire or 
standard fire exposure. Another phenomenon which has been observed in engineered timber is delamination, which is the separating of a wood layer from an adjacent one due to the failure of the adhesive. Delamination occurs when thermal penetration into the timber material interacts with an adhesive bondline. The occurrence has been observed

most specifically with CLT, due to the comparatively large weight of the lamellae and surface of exposed timber (see Su et al., 2018). There have been numerous studies on delamination in compartment fires but the occurrence of the phenomenon is variable. One study was able to show that in some cases, the delamination timing lined up with the char front reaching the first adhesive bondline (Hadden et al 2017). That study also suggested that delamination occurred when the char front had not yet reached the bondline, and the adhesive would have been at a much lower temperature than $300^{\circ} \mathrm{C}$ when it lost enough strength for the lamella to delaminate. Another study by NIST/NRC showed that CLT walls and ceilings began to delaminate variably before and after the char layer had reached the adhesive layers (Su et al., 2018).

\section{C.3.2 Timber Adhesive Standards}

A wide range of standards are currently required for high-temperature performance of adhesive in engineered timber. Figure C.1 shows a summary of timber adhesive standards in Canada from Quiquero and Gales (2017). 


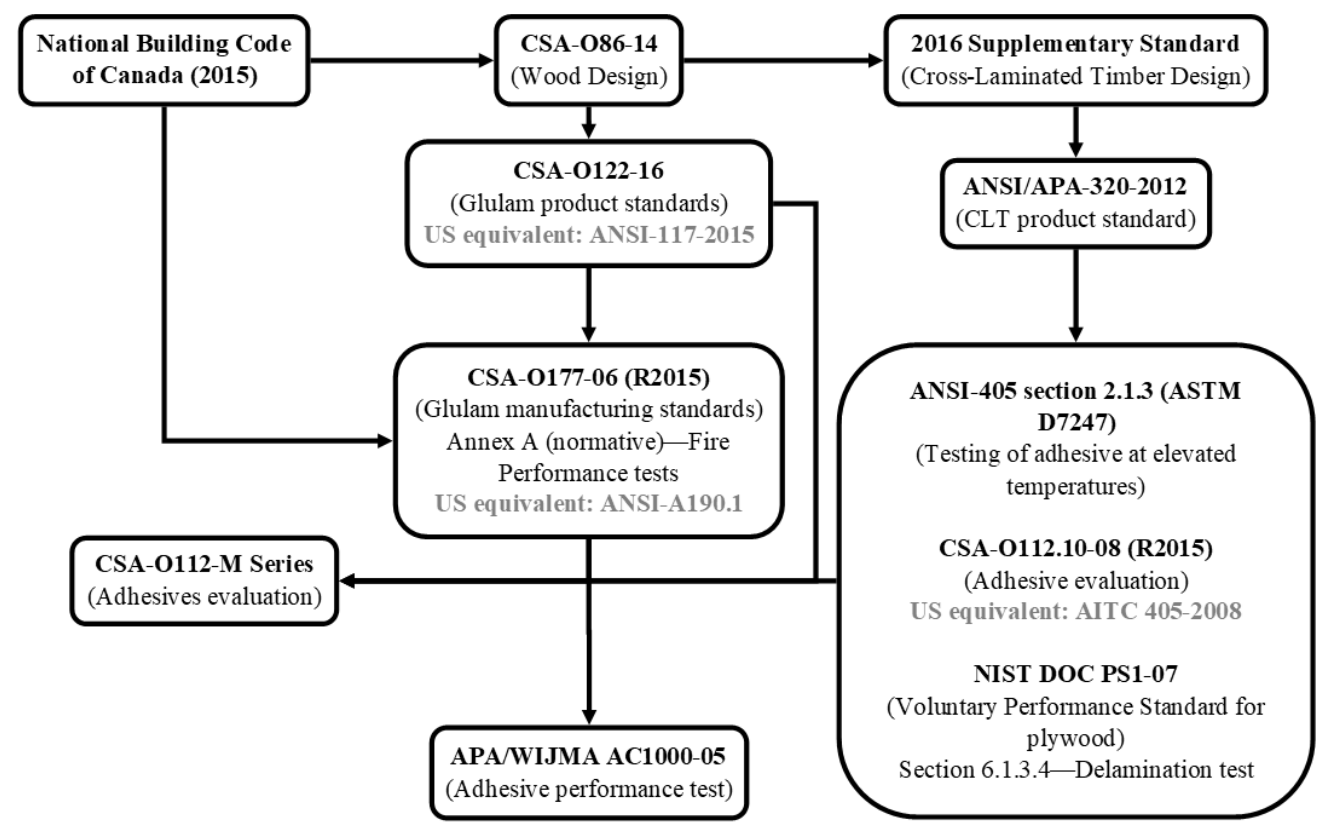

Figure C.1: Canadian timber adhesives standards summary (see Quiquero and Gales, 2017)

These standards (summarized in Figure C.1) are highly fragmented and do not necessarily test conditions that adhesive bonds in timber would be exposed to in a real fire. For example, no standard exists that evaluates the performance of adhesive bondlines beneath the char layer in burnt engineered timber samples, but rather test the strength of the adhesive under uniform heating less than the charring temperature of wood. The main test method for evaluating the strength of adhesives in these standards is a shear test by compression loading on specimens that are $50 \mathrm{~mm}$ squared by $40 \mathrm{~mm}$ thick, with a slight offset on either side of the shear plane.

\section{C.3.3 Research on Timber Adhesive Fire Performance}

No previous tests have been done, to the knowledge of the author, where timber adhesive samples being tested have been exposed to extreme heat and the char front has impinged on the glue line under examination. Adhesives in timber are typically tested at these temperatures below $300^{\circ} \mathrm{C}$ as the strength of the material is assumed to be completely lost 
at this point as the wood chars. However, this means that the performance of the adhesive just beyond a char front has not been studied. Notable studies include a study by Frangi et al. (2004) and Clauß et al. (2011). Frangi et al. (2004) tested small $40 \mathrm{~mm}$ bond lines on double lap specimens through compression loading for several different adhesives including one phenol-resorcinol-formaldehyde resin (PRF) and five different polyurethane adhesives (PUR). Hundreds of samples were heated in an oven to various temperatures ranging from ambient to $170^{\circ} \mathrm{C}$. It was found that the behaviour of the PUR varied greatly between manufacturer and thus chemical composition. Three PUR and the PRF adhesive performed similarly to that of the wood itself, while two of the PUR performed very poorly and began to lose strength from $50-70^{\circ} \mathrm{C}$. Clauß et al. (2011) similarly performed shear tests on several different adhesives but using single lap samples with bond lengths of only $10 \mathrm{~mm}$ through tensile loading. The specimens were uniformly heated to various temperatures up to $220^{\circ} \mathrm{C}$. The results in this test series were highly variable, but similarly found that the performance of various PUR adhesives were diverse with some losing thermal stability around $70^{\circ} \mathrm{C}$ while others remained stable until $150-200^{\circ} \mathrm{C}$. The most recent testing that specifically referenced to adhesive performance was a two-part test series by Nicolaidis et al. (2016) and Emberley et al. (2016) on glued single lap samples exposed to environmental chamber heating and CLT beams exposed to radiant heating, respectively. Pine and spruce wood were used, respectively, with a one-component PUR. The single lap samples had $600 \mathrm{~mm}$ bond lengths and were heated uniformly immediately prior to testing. The exposure on the CLT beams was akin to a realistic fire condition but at a very low heat flux of $6 \mathrm{~kW} / \mathrm{m} 2$, thus inducing a gradient of in-depth temperature below the pyrolysis temperature of wood. The bond lines were mostly uniformly heated 
perpendicular to the bond on the tension side of the CLT beam, increasing the temperature of the bond lines to just $60-85^{\circ} \mathrm{C}$. Heating perpendicular to the bond line is a common scenario on CLT in realistic compartments, however Glulam members will often experience heating parallel to the exposed adhesive lines. In both parts of that experimental series, changes in the failure mode from primarily timber failures to primarily adhesive failures were seen in the higher temperature range tests. Of particular note, the failure modes in the adhesive were often made more severe by the discontinuity and stress concentrations caused by timber failure propagating into an adhesive joint. This stresses the importance of the size effect in experiments and failure modes changing based on the length of bond line tested. An experimental programme was previously undertaken by the authors to research the underlying mechanics of the failure of fire-damaged engineered timber, specifically in timber box sections. The test series included axial compression of glulam coupons and four-point loading of short glulam beam sections (Quiquero and Gales, 2016; Quiquero et al., 2016). A group of the samples were burned in a furnace in a one hour standard fire, and allowed to burnout after fuel supply was halted for an additional hour. Following this the samples were allowed to cool, and tested at a later date so that the material could reacclimatize in moisture and so that the mechanics of the beam performance and failure could be closely studied. Of the samples that were not burnt, some were left as control specimens with original dimensions and a portion were manually altered to carve off the corresponding char depth to the burnt specimens. Specimens were carved to remove the char depth recorded in order to investigate whether the assumption that the cross-section below the pyrolysis zone had full strength. In all cases in which a corresponding specimen was carved to match a burnt specimen, the manually reduced test 
always had a significantly higher capacity than the burnt counterpart. This indicated that there was some other factor contributing to the failure of the specimens other than the loss of cross-sectional area. The adhesive in the engineered timber could be affected by the extreme heating and the charring encroaching on glue-lines, causing it to have a reduced strength. Samples were not of representative length $(1 \mathrm{~m})$. The study herein considers a realistically sized member, and controlled study of the strength of the adhesive layer.

\section{C.4 Experimental Methodology}

Herein a holistic test programme has been conducted to study commercially available glulam and the performance of adhesive after exposure to fire conditions. The Glulam had $5 \%$ moisture content (before heating). It was machine rated as $24 \mathrm{f}$-ES and made of SPF species. Two test phases were considered generalized by the experimental goal. For Phase 1, Glulam samples of 100 by 100 by $45 \mathrm{~mm}$ were utilised (scaled to fit a heating apparatus). For Phase 2, Glulam samples were beams of dimensions 4200 by 195 by $45 \mathrm{~mm}$. As such, the members considered herein were thin, and only moderate degrees of charring were considered.

\section{C.4.1 Phase 1: Small scale adhesive shear tests}

The shear strength of adhesive lines is often used as a performance criterion as it is the most common interfacial stress state in structural members in practice. An experimental program was developed in which over 50 samples of Glulam adhesive lines were heated under various severities. Block test specimens were prepared from full scale Glulam products. The blocks were cut from the larger Glulam billets, ensuring that an adhesive line was located exactly along the centreline of the sample for shear testing. The samples were cut around manufacturer made glue lines which used a PUR adhesive, and around lab- 
prepared PRF adhesive lines. The PRF was a prepared adhesive used as a comparator and obtained from a timber mill and suitably pressed to standard. A cone calorimeter was used as a heat source with well-defined and controlled constant incident heat fluxes. The sample was placed at a fixed distance from the conical heating coil and a shield was placed around the specimen to minimize convective heat transfer. Several experiments have been done internationally on timber specimens in cone calorimeters. The tests have shown good repeatability, especially at higher heat fluxes (from $25 \mathrm{~kW} / \mathrm{m}^{2}$ to $60 \mathrm{~kW} / \mathrm{m}^{2}$ ) and poorer repeatability at lower heat fluxes such as $10 \mathrm{~kW} / \mathrm{m}^{2}$ (Reszka and Torero, 2008). A few other notable studies which confirm the dependable performance of wood samples in a cone calorimeter include: Spearpoint and Quintiere (2001) and DiDomizio et al. (2016). The grain orientation of the wood samples was perpendicular to the applied heat flux, and the tests were run with the wood samples in a horizontal configuration. An incident heat flux of $50 \mathrm{~kW} / \mathrm{m}^{2}$ was chosen as a typical heat exposure and $30 \mathrm{~kW} / \mathrm{m}^{2}$ was chosen as a comparator. Three, six, ten and fifteen minute exposure durations were considered in order to observe the performance of the remaining adhesive under varying quantifiable char depths. The specimens were extinguished with water immediately after the heating period. Five samples from the PUR set and five samples from the PRF set were left unheated as controls. For the purposes of the study, one-sided extreme exposure was used to actually char the wood and leave an unheated wood and adhesive region below the pyrolysis zone (Table C.1). 

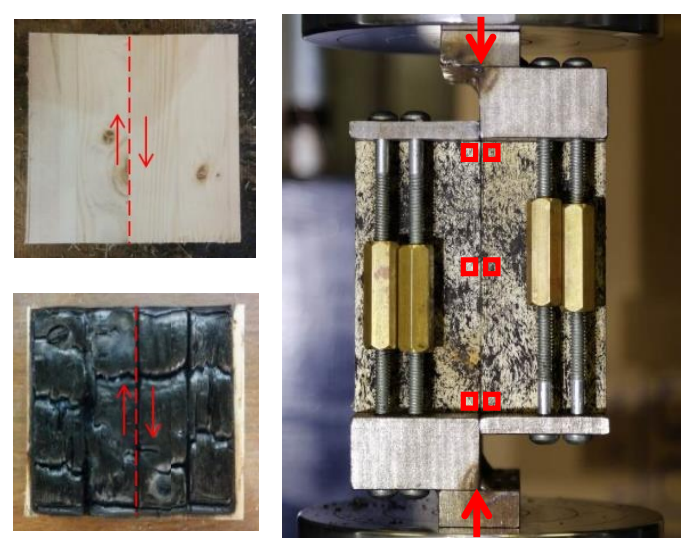

Figure C.2: Shear plane shown on unheated and heated samples (left) and shear apparatus test setup (right)

In order to create a shear test setup compatible with the sample dimension requirements for the cone calorimeter, a shear apparatus was built (see Figure C.2). The apparatus was placed between two loading plates on an INSTRON load actuator through which the wood blocks were placed under a shear force through compression loading. The loading rate was $1 \mathrm{~mm} / \mathrm{min}$. The shear plane was along the centreline of the wood specimen that corresponded to the adhesive bond to be tested, shown in Figure C.2. Slip was measured using Digital Image Correlation (described below). The failure mode is expected to be due to primarily to shearing stresses.

Table C.1: Summary of the number of samples tested for each adhesive type, heat exposure and heating duration

\begin{tabular}{|c|c|c|c|c|}
\hline \multirow{2}{*}{$\begin{array}{l}\text { Heating Duration } \\
\text { (min) }\end{array}$} & \multicolumn{2}{|c|}{$\begin{array}{l}\text { Number of PUR samples with each heat } \\
\text { flux exposure }\end{array}$} & \multicolumn{2}{|c|}{$\begin{array}{l}\text { Number of PRF samples with each heat } \\
\text { flux exposure }\end{array}$} \\
\hline & $30 \mathrm{~kW} / \mathrm{m}^{2}$ & $50 \mathrm{~kW} / \mathrm{m}^{2}$ & $30 \mathrm{~kW} / \mathrm{m}^{2}$ & $50 \mathrm{~kW} / \mathrm{m}^{2}$ \\
\hline 0 & \multicolumn{2}{|c|}{5} & \multicolumn{2}{|c|}{5} \\
\hline 3 & 3 & 2 & 3 & 2 \\
\hline 6 & 3 & 2 & 2 & 2 \\
\hline 10 & 4 & 4 & 2 & 3 \\
\hline 15 & 4 & 3 & 2 & 2 \\
\hline
\end{tabular}




\section{C.4.2 Phase 2: large scale beam bending tests}

A custom experimental test procedure was designed for the fire response tests conducted. This testing consisted of fire exposure and mechanical testing of the beams afterwards. The overall test setup, consisting of a single beam locally exposed to a fire is shown in Figure C.3. The fire for each test was fuelled by 1 litre of kerosene, contained in a meter long steel trough. The fire burned for approximately 5 minutes, producing a peak surface average temperature of $900^{\circ} \mathrm{C}$ on the exposed beam. The fire dynamics of the test procedure are not of interest as only the degree of damage state is of concern - hence the beams were protected outside their fire exposed regions to inhibit flame spread for a more quantifiable damaged area. The fire is to be considered a short period of real fire impingement on a structural element. The fire exposure was designed as non-standard to explicitly induce a controlled and quantified amount of charring on the samples from test to test.
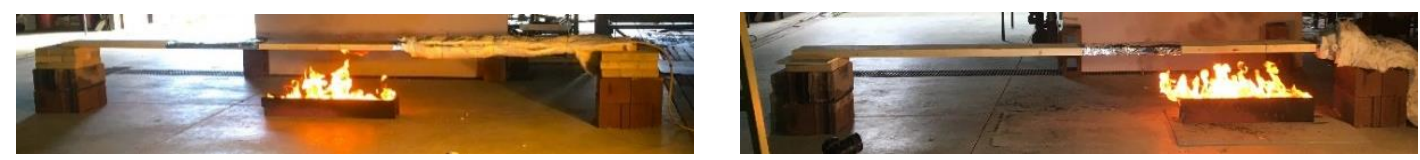

Figure C.3: Test setup of the heating portion of the experiment for the moment region (left) and the shear region (right)

Five identical Glulam beams were charred at different locations along the beam for the full duration of the fire. An additional four beams consisted of control samples and samples that would be carved to simulate charring for a total of 9 beam tests: 2 Charred and 1 Carved Mid-span; 3 Charred and 1 Carved in a side region; and 2 Control. All burnt beams were exposed on two sides along the depth of the beam in the desired charring region to provide a relatively consistent char depth. Two beams were charred directly in the center of the beam, and three were charred on one end, $270 \mathrm{~mm}$ from the edge of the beam (Figure C.3). This heating configuration had negligible differences in the amount of char observed 
$(5+/-1 \mathrm{~mm}$ at three locations on each beam equally spaced and across the depth). The heating configuration was determined through a series of test burns. The charring locations were chosen to give varied results in the loading part of the testing; where damaged shear and moment regions of the beam could be studied separately - hence the beams were not heated uniformly. Aluminum foil was wrapped around the beam immediately adjacent to the intended char zone to limit the radiant heat and flame spread to other parts of the beam. When the pool fire burned out, the beams were left to self-extinguish. Since this happened immediately, no water was used (see Section C.6 for discussion). The beams were then flipped and the heating was repeated for the other side. This test setup allowed for twosided heat exposure, a notable difference from the one-way heating performed on the small scale tests as described in Section C.4.1. An area of cross section equivalent to the charred area on the five beams that underwent the fire testing was removed from two of the noncharred beams to facilitate comparison of strength of the beams. The area removed was approximately $5 \mathrm{~mm}$ deep on all four exposed sides and $1 \mathrm{~m}$ long, in the same locations as were charred during the fire tests (either in the moment region or the shear region). All beams therefore had the same non-charred cross-sectional area for the mechanical testing. In this manner, any variation in the strength data will be due to factors other than the effective cross section reduction of the charred beams. The intention of this charring degree $(5 \mathrm{~mm})$ is not to be thought of as the authors providing information on timber's fire resistance, but rather a controlled set amount of damage that can allow the underlying breakdown mechanisms of timber exposed to fire to be rationally studied. After time for re-acclimatizing to lab moisture conditions, $5 \%$, four-point loading was performed to 
induce constant moment and shear on the specimens. Beams were restrained against lateral torsion. The loading setup can be seen in Figure C.4.

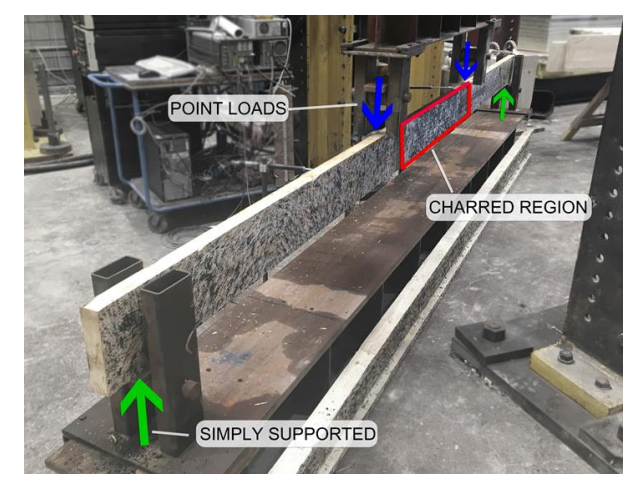

Figure C.4: Mechanical loading of simply supported beam with four point loads.

\section{C.4.3 Deformation Measurement}

Digital Image Correlation (DIC) was used to monitor the deformations of all experiments. This has previously proven accurate for measuring displacements and strain in wood specimens (see Quiquero and Gales, 2016). The displacement measurement technique uses a pixel-tracking software to locate user-specified locations on a series of high-resolution photographs. A speckled pattern is painted on the surface in order to ensure there is a very unique patch of pixels for the software to track from image to image. The software then records the location of the pixel patch in each photograph, which may be used to compute the displacement and relative deformations. In Phase 1 tests, the slip was measured at 3 points along the shear line. A sample of the pixel patches used to track the slip are shown in the right-hand image in Figure C.2. In Phase 2 tests, the centre point deflection of the beam was measured. This was complemented using linear potentiometers to record out of plane distortion. The pixel-tracking software that was used to process the test images was GeoPIV RG (Stainer, 2015). A Canon Mark III 5D DSLR camera was used in conjunction at 3 second intervals. 


\section{C.5 Experimental Results}

The results of the fire exposure and subsequent mechanical loading of the Glulam specimens are discussed below for Phases 1 and 2.

\section{C.5.1 Phase 1: Small scale adhesive shear tests}

The resulting char quantifications show predictable results however, the mechanical results had high variance between the repeated samples. This is expected due to the natural complexity and non-homogeny of wood and the small size of the samples tested.

\section{C.5.1.1 Post-fire damage state}

For the $30 \mathrm{~kW} / \mathrm{m}^{2}$ incident heat flux exposure, flaming ignition was not seen in the majority of the samples. Rather, smouldering was observed without flaming. For the $50 \mathrm{~kW} / \mathrm{m}^{2}$ incident heat flux exposure, flaming ignition in all samples was seen after around 30-40 seconds of exposure. After the samples were broken in half by shear testing, the average char depth at the centreline was also measured. The char depth was taken as the average of three points along the centreline (typically $1 \mathrm{~mm}$ of each other). The char depths of each sample versus their heating durations is plotted in Figure C.5. It can be observed that the char depths for the higher heat exposure were typically higher than those of the lower heat exposure. The trend for both the $50 \mathrm{~kW} / \mathrm{m}^{2}$ and $30 \mathrm{~kW} / \mathrm{m}^{2}$ exposures decreased in slope with longer heating durations, while this is more pronounced in the higher heat exposure. This indicates that the charring rates slow over time, as the char layer builds up and inhibits heat transfer as is expected. The higher heat flux charring rate trend tended to approach the lower heat flux constant rate, around a minimum value of $0.75 \mathrm{~mm} / \mathrm{min}$. This indicates that this might be a minimum rate of charring that may be seen in timber above the pyrolysis temperature for these incident heat fluxes. This corresponds well with recommended 
charring value of $0.7 \mathrm{~mm} / \mathrm{min}$ for standard fire exposures. Overall, the charring behaviour of the samples was very uniform and predictable. Char depths seen were close to uniform along the surface of the specimens.

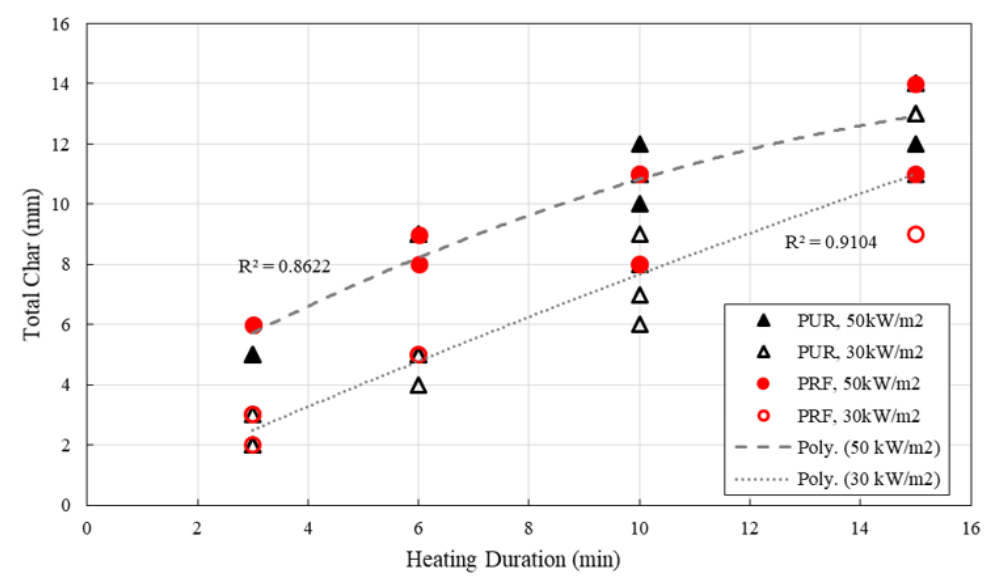

Figure C.5: Total char depths measured on samples versus the heating duration

\section{C.5.1.2 Post-fire mechanical behaviour}

The area beneath the char layer was used to calculate the failure stress of each sample at the peak load reached in the test before failure. If the adhesive within the wood region below the char front of the samples was also unharmed, the failure stress of each sample should be equal (although the peak load would be lower due to loss of cross-sectional area). However, a general decreasing trend in adhesive strength with heating duration was seen. This indicates that the adhesive beyond the char layer is affected by the heat and may have lost some additional strength unaccounted for in the sacrificial char method. A correlation is made between the remaining shear capacity of the samples and their respective remaining shear area. In theory, if the adhesive held $100 \%$ of its strength after heat exposure, these two values would correlate exactly. That is, if a sample had $80 \%$ of its shear area left after heating, the remaining shear capacity should be $80 \%$. This is shown as the red dashed line in Figure C.6. Any sample falling below this line represents a sample that did not retain its 
full adhesive strength - that is, the loss of shear capacity was greater than the loss of area. The figure illustrates a large amount of variance in the capacity of the samples. It can be seen that the majority of the samples fell well under the perfect correlation line, with most samples having a remaining area beneath the char of above $60 \%$, but many samples falling to as low as $30 \%$ remaining capacity. On average, the samples had a remaining capacity $32 \%$ below their remaining shear area. Further, the capacity of the PRF samples fell an average of $40 \%$ below their remaining area while the PUR samples were $26 \%$ below. This could indicate a difference in the adhesives or in the way they were prepared, with the PUR bonds being produced by the manufacturer while the PRF bonds were produced in the lab.

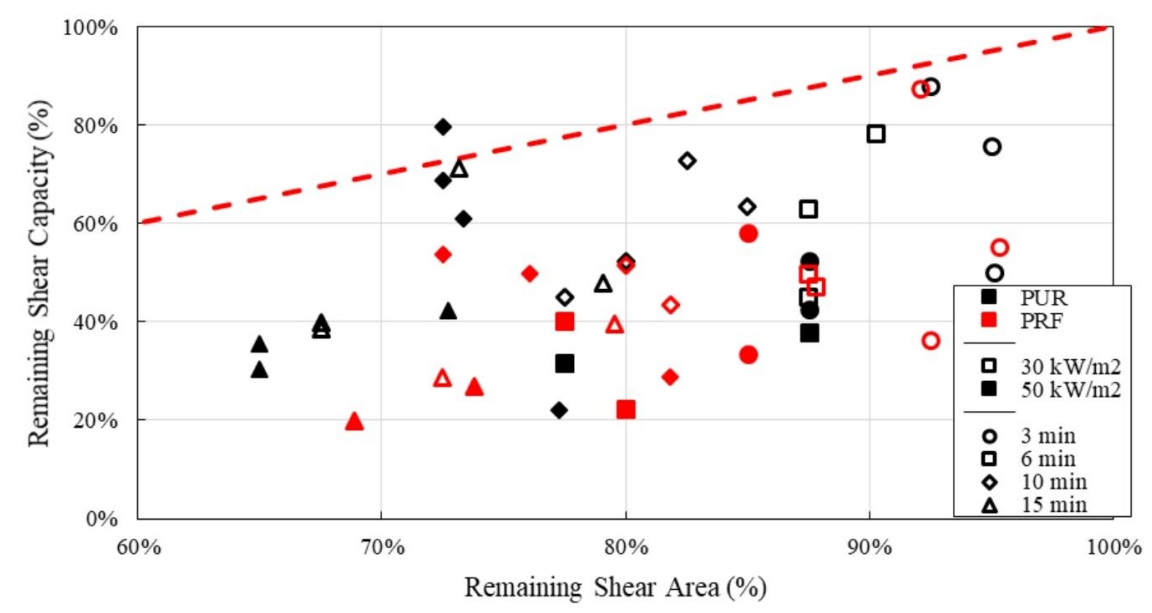

Figure C.6: Comparison of remaining shear capacity versus area for all samples Additionally, from Figure C.6, there is no clear trend or distinction developed within any of the heating durations or exposures, though it is clear that the adhesive performance falls below that of which should be expected. A comparison was made with the remaining capacity that the standard recommended sacrificial charring method would be predicted, using a charring rate of $0.7 \mathrm{~mm} / \mathrm{min}$ plus a zero-strength layer of $7 \mathrm{~mm}$. It should be noted, however, that these recommended charring rates are not validated for exposures other than a standard-fire, and are thus not totally applicable to a constant heat flux radiant exposure, 
but are included here for comparison (as it was observed to closely correlate to that seen for a $50 \mathrm{kw} / \mathrm{m}^{2}$ exposure as stated above). As seen in Figure C.7, when compared with the actual remaining shear capacity, the standard's predictions still fall significantly below the correlation line. This new data set is plotted in grey in Figure C.7 compared to the original data set in black.

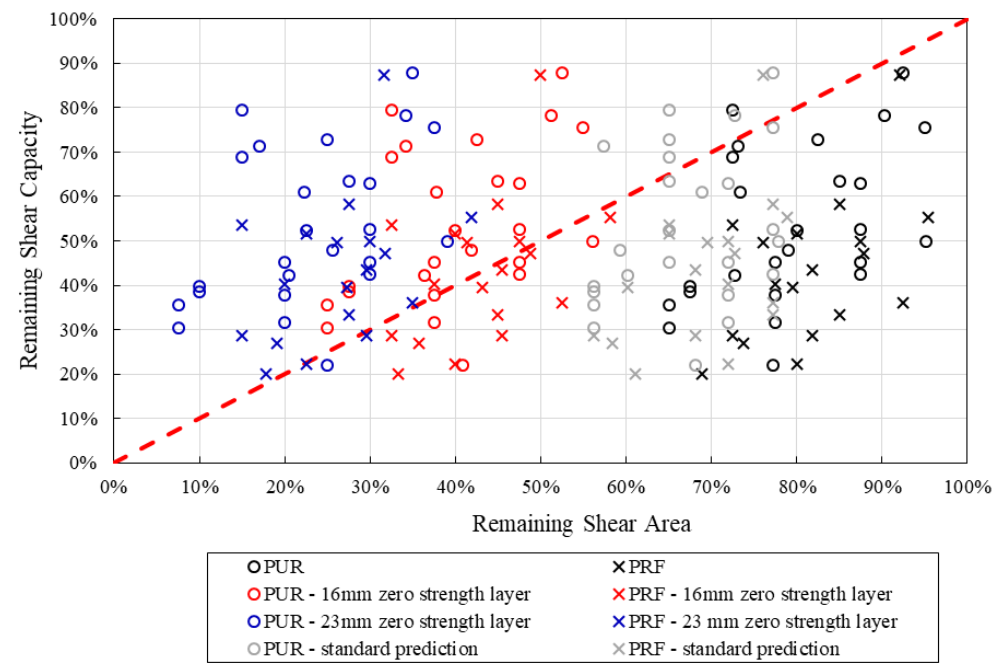

Figure C.7: Remaining shear capacity of samples versus different predictions of actual remaining shear area at full adhesive strength, compared with the measured char depths and the standard predicted sacrificial char method

There may be two possible causes for the reduced strength of the samples. The first is that, due to the small thickness of the samples, all of the adhesive beyond the char layer may be reduced in strength by a certain percentage corresponding to the lower capacities mentioned above. However, since the adhesives must have been tested to the standards discussed earlier in the manuscript, the adhesive should retain full strength for temperatures below the charring temperature of wood. Secondly, the adhesive may be full strength at a certain depth beyond the char layer, but an additional layer of adhesive beyond the encroached char front may have been degraded. In other words, a larger zero-strength layer may be present than is recommended in standards. It is likely that the adhesive and 
therefore the combined strength of the engineered wood product may have degraded to a certain depth beyond the char front, after which the remaining adhesive there may be assumed to have full strength. This effect is investigated further below. Another factor that may contribute is the loss of strength of the wood above $100^{\circ} \mathrm{C}$, as many of the failures were a combination of wood and adhesive failure. If the adhesive is assumed to have full strength at a certain depth beneath the char layer, then the area of that full strength zone may be computed using the peak shear capacity of each sample. It was assumed that this full-strength zone spans the full height of the samples, and that the width is measured through the thickness of the sample from the unheated face. In this way, the penetration of the zero-strength zone may be calculated and compared with the char layer measurement. On average, the zero-strength layer penetrated $13.3 \mathrm{~mm}$ beyond the base of the char zone. Separated for each adhesive, this value was an additional $16.2 \mathrm{~mm}$ for the PRF samples and $10.7 \mathrm{~mm}$ for the PUR samples. As a comparison, $16 \mathrm{~mm}$ was added to the measured char depth of each sample and a new remaining shear area was calculated. This new data set may also be compared to the original and standard-predicted data sets in red in Figure C.7. It can be seen that increasing the zero-strength zone to $16 \mathrm{~mm}$ below the measured char depth of the samples results in a much better prediction of the remaining strength, with the majority of the samples falling over the line. A more conservative value of $23 \mathrm{~mm}$ below the char depth could be used to give a 95th percentile of samples falling above the line, which is shown as the blue series in Figure C.7. The samples tested were very small scale, so larger scale members should be tested. A greater area of unheated wood behind the char layer would likely increase the remaining capacity of the wood so such a drastic addition to the zero-strength layer may not be required. Additionally, effects introduced by 
cracks propagating into the adhesive layer and introducing stress concentrations has been found in the past to be a large influence on adhesive failure and cannot be ignored.

In addition to the strength performance of the specimens, digital image correlation was used to record the displacements during loading. The slip was calculated using the procedures outlined in Section C.4.3 and was averaged over the three measurement locations to represent the average slip of the adhesive line. A comparison is made between the load-slip behaviour of several representative samples under $50 \mathrm{~kW} / \mathrm{m}^{2}$ incident heat exposure with PUR and PRF adhesives in Figure C.8. It is clearly observed in the figure that as the heating duration increased, lower loads were reached for the same level of slip. This indicates that the adhesive stiffness or rigidity has degraded over increased periods of heating.

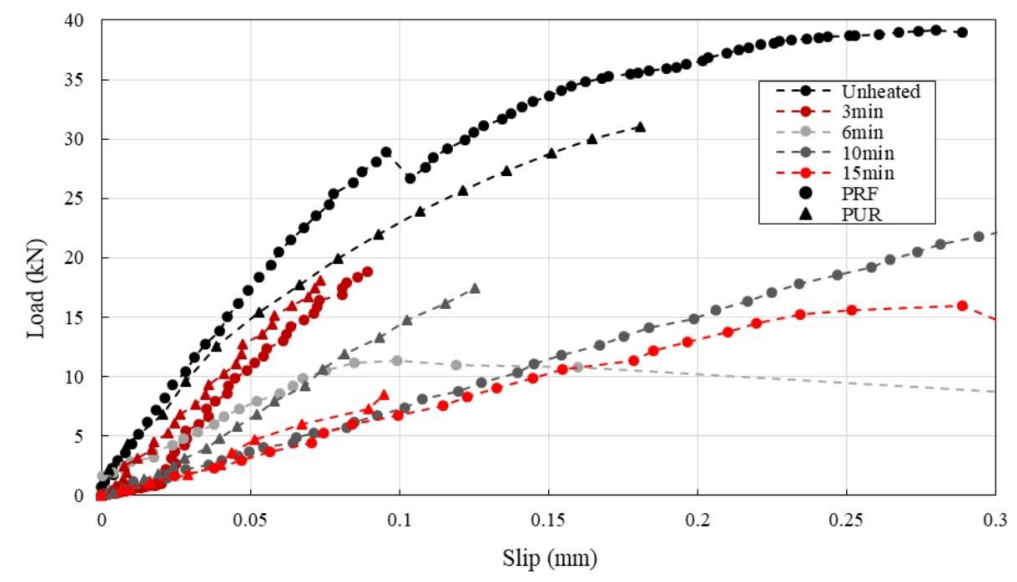

Figure C.8: Load versus slip behaviour for heated for different durations under a 50 $\mathrm{kW} / \mathrm{m}^{2}$ incident heat flux

\section{C.5.2 Phase 2: Large scale beam bending tests}

The resulting flaming behaviour and charring of the burnt beams was consistent through all five exposed samples. Variability cannot be quantified in this phase as a smaller number of beams were tested due to the larger scale. However, the results of the burnt beams are 
compared herein to the unaltered control beams of original dimensions and two beams with manually reduced cross-sections in critical regions.

\section{C.5.2.1 Post-fire damage state}

To adequately quantify the degree of charring so that the samples could be adequately carved, the charred and damaged samples were tested first in four-point bending. After testing, the beams were then sliced in the burnt region and the charring depths were measured across the cross section $(5+/-1 \mathrm{~mm})$, where it was confirmed that there were minimal differences in the char depths observed. $5 \mathrm{~mm}$ was used for the depth of all carving on the sides of the beams. Carving was performed manually through a plane process and confirmed afterwards.

\section{C.5.2.2 Post-fire mechanical behaviour}

The average failure loads obtained during the mechanical loading are summarized in Figure C.9. On an average basis, the control beams failed at the highest applied load, with all of the carved and charred beams failing at lower loads. All failure loads were higher than the hand calculated estimated strength, accounting for only the reduction in cross sectional area (predicted as $10.6 \mathrm{kN}$ ). The displacement of the beams is presented in the plots of load versus vertical displacement in Figure C.10. Displacements were measured by digital image correlation. In all tests lateral movement, by linear potentiometers (an indication of torsional failure) was negligible. 

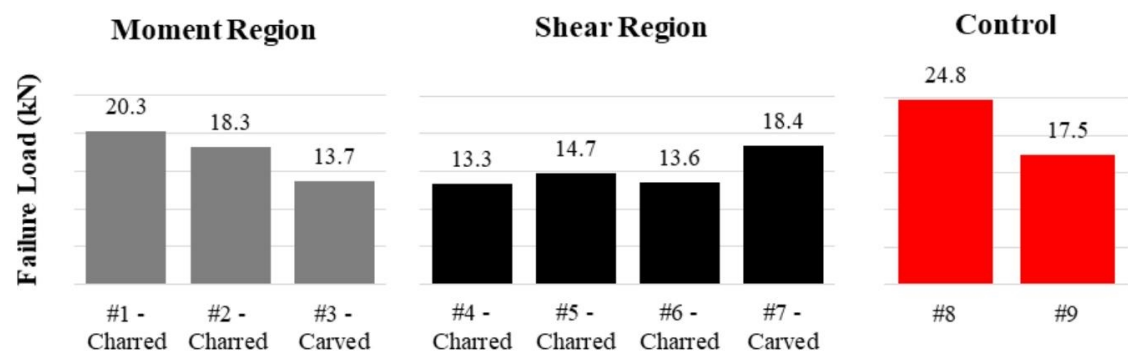

Figure C.9: Failure load of all beams damaged in the moment region, shear region, as well as the control beams
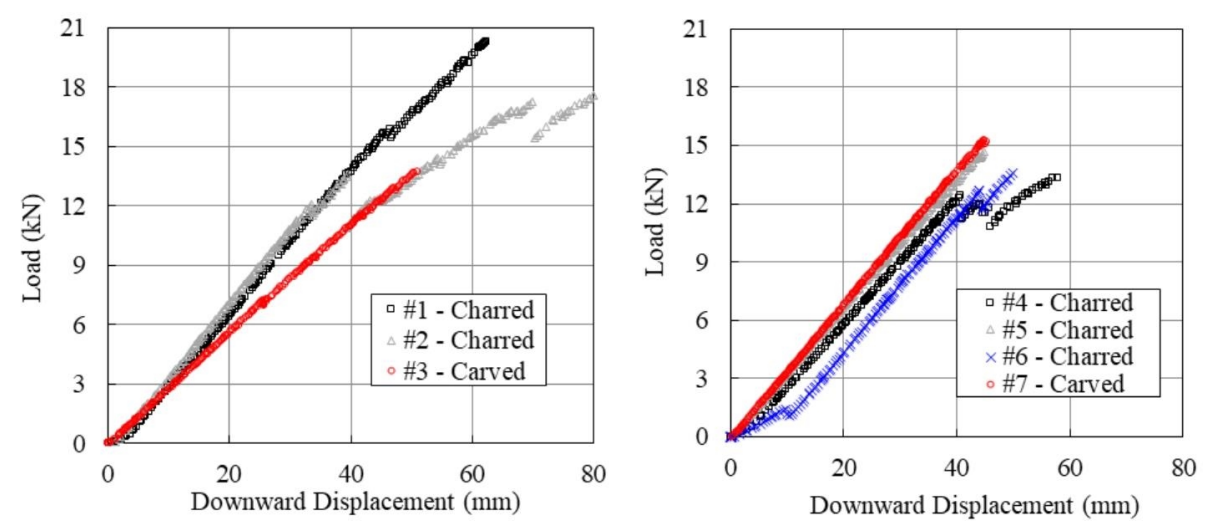

Figure C.10: Load versus downward displacement beams damaged in the moment region (left) and shear region (right)

The two control beams failed at relatively high loads with the difference possibly due to inherent material variability between the two specimens. Amongst beams damaged in the moment region (midspan), the results were quite variable; the beam with mechanically reduced cross section failed at a relatively low load compared to the charred specimens, which withstood relatively high loads, losing only $8.9 \%(1.9 \mathrm{kN})$ of their capacity. It is possible that the carved beam had a material defect that was not visibly apparent, causing the low failure load. A large reduction in moment capacity was not expected from the exposure as the depth of the cross-section was largely unaltered, contributing to most of the moment capacity. Regarding beams damaged in the shear region, the beam that had its cross section mechanically reduced through carving displayed the highest stiffness, as the slope of its load-displacement curve is the greatest, and experienced a higher failure load 
than any of the charred beams. The beams burnt in the shear region failed at relatively low yet consistent values of load, while the mechanically carved beam performed better, and failed at a higher load. The CSA O86-14 guidance predicts a failure load of $10.6 \mathrm{kN}$ for a cross section reduced by $5 \mathrm{~mm}$ on two sides of the beam (which is the damage state that was observed). All of the shear damaged beams observed a higher failure load than this prediction. The charred beams failed on average at a load $24.6 \%$ lower than the beam that has been mechanically carved in the shear region. Since the beams that were damaged through charring had the same cross section as the beams that were damaged through mechanical carving, this difference is indicative of adhesive degradation. This is in line with the loss of strength observed in Phase 1 of the testing and the previous research discussed in Section C.3.3. To achieve a predicted failure load for the charred beams $24.6 \%$ lower than the predicted load of the carved beam, the equivalent cross section of the beam would be reduced further by $4.3 \mathrm{~mm}$ on both sides (beyond the reduced dimensions of the carved beam). This reduction in area would be considered to be due to degradation effects (that is, a zero strength layer), as the char depth has already been accounted for in the reduction in area of the carved beam. Continuing to examine the beams charred in the shear region, the burnt beams failed at a load 34.5\% lower than the control beams. A reduction in cross sectional area of $7.8 \mathrm{~mm}$ on two sides of the beam would be required to generate a predicted value $34.5 \%$ lower than the predicted failure load of the undamaged beams. Examining standard allowances, and in particular CSA O86-14, the amount of cross sectional area lost to charring and other effects is calculated first by determining the char depth, and then adding allowances for the zero strength layer. The remaining area is assumed to retain its full, initial strength. Annex B.4.2 quantifies the rate of char of Glulam 
as $0.70 \mathrm{~mm} / \mathrm{min}$, indicating that at five minutes of exposure the char depth would be calculated as $3.5 \mathrm{~mm}$. As previously mentioned, this standard guidance is not entirely applicable to beams damaged as per the procedure used for these tests (described in Section C.4.2), but for comparison purposes is still considered. In addition to the calculated char depth, Annex B.5 quantifies the zero strength layer for exposure times less than 20 minutes as being interpolated linearly between $0 \mathrm{~mm}$ and $7 \mathrm{~mm}$, depending on the time exposed. At five minutes of exposure, this would predict the zero strength layer as being $1.75 \mathrm{~mm}$, meaning that the total calculated depth lost due to char and the zero strength layer would be $5.25 \mathrm{~mm}$. In order to create a loss of depth of $7.8 \mathrm{~mm}$, the zero strength layer would need to be calculated as $4.3 \mathrm{~mm}$ rather than $1.75 \mathrm{~mm}$ (assuming the predicted char depth is correct). This value of $4.3 \mathrm{~mm}$, which would be considered to be due to degradation effects, is the same depth that has been found to be compromised in the comparison of the charred and carved shear region beams. To achieve a zero strength layer of $4.3 \mathrm{~mm}$, the depth of the zero strength layer must be interpolated linearly between $0 \mathrm{~mm}$ and $17.2 \mathrm{~mm}$ rather than $7 \mathrm{~mm}$ (for exposure times less than 20 minutes). This suggested value of 17.2 $\mathrm{mm}$ implies that the depth of the zero-strength layer proposed in Section C.5.1 (23 $\mathrm{mm}$ past the char depth) would be a conservative value when applied to these large-scale beams.

\section{C.6 Future Research}

In Canada, heavy timber construction is permitted currently in mid-rise buildings by code and larger structures are already planned to having prescriptive code approval. However, with heavy timber construction comes large Glulam (or other engineered timber) structural elements which have the potential to be exposed to fire in an extreme scenario. In larger members, it has been seen in previous research that material defects or timber cracks 
propagating into adhesive lines have significant potential to govern the critical failure of these elements. The stress concentrations from these defects has been observed to induce delamination of adhesive layers in heated engineered timber and is of the utmost importance to be studied in the near future.

There is one significant difference when considering Phase 1 and Phase 2. Phase 1 had significant water suppression opposed to Phase 2 that had none. The authors believe that this fact is plausibly responsible for the differences observed in the zero strength calculations and this aspect requires additional study. There is no question in a real fire water will be utilised to suppress a fire in a timber building (exposed or not) and this can complicate the degree of damage to adhesives that are in a liquid state, and may re-solidify during the cooling process. Nevertheless, the large scale beams do yield confidence that for short term exposures without water suppression, the adhesive degradation will be of minor effect in comparison to the small scale samples with short term heat exposure and with water suppression. Further testing to investigate the influence of the water suppression on the results should be done.

While self-extinguishment was observed in the beam samples, it will be necessary to study this phenomenon to a greater degree where fire spread is allowed. A comprehensive listing of future research needs for enabling high rise timber beyond adhesive degradation is provided in Jeanneret et al. (2017) which the reader is encouraged to consult, having been derived from Yang et al (2015).

To study the effect of longer term exposure it is necessary to scale the cross-sections up to representative sizes for mass timber (a min of $190 \mathrm{~mm}$ x $190 \mathrm{~mm}$ ). This will also allow discussion into real specimen section behaviour and direct conclusions may be drawn about 
realistic performance. Until these tests are performed there is legitimate concern in the authors' opinion that a prescriptive rate for the zero strength layer criterion of $7 \mathrm{~mm}$ for engineered Glulam may not necessarily be conservative. These future tests may allow a conclusive solution or criterion to be drawn, however for now a range should be expected that depends on more factors than just the type of heating as discussed in other literature. Factors including member size, adhesive composition, heating duration and exposure should be considered for all types of engineered timber.

While the work done herein has been compared to prescriptive code methods of predicting the capacity of heavy timber structural elements in fire, the suggested increase in zero strength layer is drastic and likely largely over-conservative. The results have shown, however, that the behaviour is so variable and highly complex that a very conservative prescriptive approach would be required. However, finite element modelling of timber proponents may be used to more accurately predict the behaviour of such components if an appropriate model is used. Computer modelling and analysis should be developed and validated incorporating the effects of these findings in order to accurately predict the behaviour of complex engineered timber structures.

It should also be noted that the authors only considered one type of adhesive in manufactured Glulam, along with their own lab grade adhesive. Additional adhesives by various manufactures and of different compositions should also be considered, and it should be recognized that there currently is significant research being performed into advancing adhesives so that they do not degrade to the same degree in high temperatures seen in fires. The smaller scale samples would be highly influenced by any small defects or gaps within the adhesive layers, the critical effects of which would be amplified. This 
was seen as the effect was less in the larger beams tested and should be investigated further with more repeated tests and different adhesives.

\section{C.7 Conclusions}

Due to the drivers towards massive engineered timber construction in Canada as of late, it is paramount to delve deep into the details of the material's performance in and after fire scenarios. Studying the material post-fire damage (as opposed to in-fire) allows details in the mechanics of the material's behaviour and failure to be observed. Additionally, the resilience of massive timber post-fire is an important topic to be discussed. The opportunity to have the capability to build with and understand materials that are resilient to fire and how to rehabilitate and reuse the structures after such an event also holds immense merit in the realm of property value, business continuity and insurance. If Canada is to build exemplar structures with engineered timber exposed, having confidence in having the material exposed is invaluable.

In the small scale testing, although the high variance in the results typical of wood was seen, some clear trends emerged in the strength and slip properties of the adhesive with varying heat exposures. A clear downwards trend was seen in the strength of all adhesive samples when plotted against heating duration, both for the $30 \mathrm{~kW} / \mathrm{m}^{2}$ and the $50 \mathrm{~kW} / \mathrm{m}^{2}$ heating regimes. By only considering the charred layer or the sacrificial char layer from recommendations, the remaining full-strength zone and thus the predicted remaining capacity of all specimens was greatly over-estimated. On average, an added zero strength layer of $16 \mathrm{~mm}$ beyond the recorded char depth was required, with a conservative $95^{\text {th }}$ percentile zero strength layer being $23 \mathrm{~mm}$ beyond the char front. The slip behaviour also 
exhibited trends of increased slip with load on the samples. This indicates that the heated adhesive loses some stiffness compared to the unheated samples.

In regards to the beam testing, it was clear that the charring reduced the strength of the beams when compared to the control samples. The moment damaged beams, which failed at an average of $19.3 \mathrm{kN}$, exhibited only a slight reduction in comparison to the control beams, which failed at an average of $21.1 \mathrm{kN}$. The beam that was mechanically reduced in its moment region failed at a lower load than both the charred beams and the control beams, at a load of $13.7 \mathrm{kN}$. Conclusions that may be drawn from the examination of the moment beams are, firstly, there is only a relatively small reduction in strength between the control beams and the charred beams. This should be expected, as the beams were charred on their long side, but tested in bending standing vertically. Bending is more impacted by depth and section modulus, while shear is more impacted by cross-sectional area. As expected, the bending capacity was not be impacted much as the depth didn't see much reduction, and the carved specimen may have been an outlier due to a material defect.

Unlike the beams damaged in the moment region, the beams charred in the shear region had a significant reduction in strength in comparison to the control beams. The beams charred in the shear region failed at an average of $13.9 \mathrm{kN}$, notably lower than both the control beams peak load of $21.1 \mathrm{kN}$ and the mechanically carved beam of $18.4 \mathrm{kN}$. This represents approximately a $35 \%$ reduction in strength of the burnt beams compared to the control beams, and a $25 \%$ reduction compared to the carved counterpart. In all instances, the beams failed at a higher load than directly predicted by current guidance, in particular CSA 086-14 equations for Glulam. However, in examining reduction in strength of the 
charred shear region beams in comparison to the carved beam, the determination of the zero-strength layer may not fully account for all degradation effects that are occurring.

Both the small scale and large scale testing showed that the current guidance that exists for approximating the zero strength layer $(7 \mathrm{~mm})$, may in some instances be under conservative. These may be effected by suppression operations along with fire type and duration, and the size and defects of the specimens. It is recommended by the authors, that until a more holistic database of tests is established, conservative approximations be used in calculations involving exposed engineered timber with adhesives $(23 \mathrm{~mm})$.

\section{C.8 Acknowledgments}

NSERC discovery grant. The authors thank Chloe Jeanneret, Georgette Harun, Beth Weckman, and Matt Smith.

\section{C.9 References}

CSA O86-14. (2016). Engineering design in wood, CSA Group, Mississauga, Canada.

DiDomizio, M. J., Mulherin, P., and Weckman, E. J. (2016). "Ignition of wood under timevarying radiant exposures." Fire Safety Journal 82. 131-144.

Emberley, R., Nicolaidis, A., Fernando, D., Torero, J. L. (2016). “Changing Failure Modes of Cross-Laminated Timber." Structures in Fire, Proceedings of the $9^{\text {th }}$ international conference, New Jersey, USA. 643-649.

Frangi, A., Fontana, M. and Mischler, A. (2004). "Shear behaviour of bond lines in glued laminated timber beams at high temperatures." Wood Science and Technology 38. 119-126. doi:10.1007/s00226-004-0223-y 
Gales, J., Zhou, A., Smith M., Braxton, N., Smith, C., and LaMalva, K. (2018). Chapter 11: Acceptance Criterion, Structural Fire Engineering Manual of Practice, ASCE, Virginia, USA. 15 pp.

Hadden, R. M., Bartlett, A. I., Hidalgo, J. P., Santamaria, S., Wiesner, F., Bisby, L. A., Deeny, S., Lane, B. (2017). "Effects of exposed cross laminated timber on compartment fire dynamics.” Fire Safety Journal 91. 480-489.

Hopkin, D., Schmid, J., Friquin, K. (2016). “Timber Structures Subject to Non-Standard Fire Exposure - Advances and Challenges.” WCTE. 12 pp.

Jeanneret, C., Smith, M., and Gales, J. (2017). "Fire safety towards enabling timber structures in Canada.” Applications of Structural Fire Engineering.

Lange, D., Boström, L., Schmid, J., Albrektsson, J. (2015). “The Reduced Cross Section Method Applied to Glulam Timber Exposed to Non-standard Fire Curves.” Fire Technology 51. 1311-1340. doi:10.1007/s10694-015-0485-y

National Research Council of Canada, Canadian Commission on Building and Fire Codes. (2015). National Building Code of Canada, National Research Council of Canada, Ottawa, ON.

Nicolaidis, A., Emberley, R., Fernando, D., Torero, J. L. (2016). “Thermally Driven Failure Mode Changes in Bonded Timber Joints.” Proceedings of World Conference on Timber Engineering, WCTE 2016, Vienna, Austria.

Quiquero, H., Gales, J., and Hadjisophocleous, G. (2016). "Behaviour of Char Layer in Fire-Damaged Box Section Timber Beams.” Interflam 2016. 1063-1074. 
Quiquero, H., and Gales, J. (2017). “Comparing timber adhesive shear strength properties after fire damage.” Fire and Materials, California, USA. 556- 566.

Reszka, P. and Torero, J. L. (2008). "In-depth temperature measurements in wood exposed to intense radiant energy." Experimental Thermal and Fluid Science 32. 1405-1411. doi:10.1016/j.expthermflusci.2007.11.014

Spearpoint, M. J. and Quintiere, J. G. (2001). "Predicting the piloted ignition of wood in the cone calorimeter using an integral model - effect of species, grain orientation and heat flux." Fire Safety Journal 36. 391-415.

Stanier, S.A., Blaber, J., Take, W.A. and White, D.J. (2015). "Improved image-based deformation measurement for geotechnical applications." Canadian Geotechnical Journal. 53(5): 727-739

Su J., Lafrance P.S., Hoehler M., and Bundy M. (2018). Fire Safety Challenges of Tall Wood Buildings- Phase 2: Task $2 \& 3$ - Cross Laminated Timber Compartment Fire Tests. FPRF. 397 pp.

Yang, J. C. et al. (2015). International R\&D Roadmap for Fire Resistance of Structures: Summary of NIST/CIB Workshop, National Institute of Standards and Technology, Maryland, USA. 


\section{Appendix D - Adhesive Testing Data and Zero Strength}

\section{Layer Calculations}

\section{D.1 PRF Samples}

\begin{tabular}{|c|c|c|c|c|c|c|c|c|}
\hline \multicolumn{7}{|c|}{ Sample Information } & \multicolumn{2}{|c|}{$\begin{array}{c}\text { Recorded } \\
\text { Data }\end{array}$} \\
\hline Sample & Type & $\begin{array}{l}\text { Heating } \\
\left(\mathbf{k W} / \mathbf{m}^{2}\right)\end{array}$ & $\begin{array}{l}\text { Duration } \\
\text { (min) }\end{array}$ & $\begin{array}{c}\text { Original } \\
\text { Thickness } \\
(\mathbf{m m})\end{array}$ & $\begin{array}{c}\text { Sample } \\
\text { Height } \\
\text { (mm) }\end{array}$ & $\begin{array}{c}\text { Original } \\
\text { Shear } \\
\text { Area } \\
\left(\mathbf{m m}^{2}\right)\end{array}$ & $\begin{array}{c}\text { Peak } \\
\text { Load } \\
(k N)\end{array}$ & $\begin{array}{l}\text { Char } \\
\text { depth } \\
(\mathrm{mm})\end{array}$ \\
\hline 1 & PRF & 0 & 0 & 40 & 100 & 4000 & 31.03 & 0 \\
\hline 2 & PRF & 30 & 3 & 40 & 100 & 4000 & 11.19 & 3 \\
\hline 3 & PRF & 30 & 3 & 43 & 100 & 4300 & 18.41 & 2 \\
\hline 4 & PRF & 30 & 3 & 38 & 96 & 3648 & 24.70 & 3 \\
\hline 5 & PRF & 30 & 6 & 40 & 100 & 4000 & 15.44 & 5 \\
\hline 6 & PRF & 30 & 6 & 41 & 100 & 4100 & 14.98 & 5 \\
\hline 7 & PRF & 30 & 10 & 40 & 100 & 4000 & 16.00 & 8 \\
\hline 8 & PRF & 30 & 10 & 44 & 100 & 4400 & 14.82 & 8 \\
\hline 9 & PRF & 30 & 15 & 40 & 99 & 3960 & 8.803 & 11 \\
\hline 10 & PRF & 30 & 15 & 44 & 100 & 4400 & 13.48 & 9 \\
\hline 11 & PRF & 50 & 3 & 40 & 100 & 4000 & 18.06 & 6 \\
\hline 12 & PRF & 50 & 3 & 40 & 100 & 4000 & 10.39 & 6 \\
\hline 13 & PRF & 50 & 6 & 40 & 100 & 4000 & 6.893 & 8 \\
\hline 14 & PRF & 50 & 6 & 40 & 100 & 4000 & 12.50 & 9 \\
\hline 15 & PRF & 50 & 10 & 40 & 100 & 4000 & 16.65 & 11 \\
\hline 16 & PRF & 50 & 10 & 44 & 99 & 4356 & 9.712 & 8 \\
\hline 17 & PRF & 50 & 10 & 46 & 100 & 4600 & 17.75 & 11 \\
\hline 18 & PRF & 50 & 15 & 42 & 101 & 4242 & 8.861 & 11 \\
\hline 19 & PRF & 50 & 15 & 45 & 100 & 4500 & 6.963 & 14 \\
\hline
\end{tabular}




\begin{tabular}{|c|c|c|c|c|c|c|c|}
\hline & \multicolumn{4}{|c|}{ Char Calculations } & \multicolumn{3}{|c|}{ Strength Calculations } \\
\hline Sample & $\begin{array}{c}\text { Averaged } \\
\text { Charring } \\
\text { Rate } \\
(\mathrm{mm} / \mathrm{min})\end{array}$ & $\begin{array}{c}\text { Thickness } \\
\text { below } \\
\text { char } \\
\text { layer } \\
\text { (mm) }\end{array}$ & $\begin{array}{c}\text { Remaining } \\
\text { Shear } \\
\text { Area } \\
\left(\mathbf{m m}^{2}\right)\end{array}$ & $\begin{array}{c}\text { Shear } \\
\text { Area } \\
\text { Ratio }\end{array}$ & $\begin{array}{c}\text { Failure } \\
\text { Stress } \\
\text { (MPa) }\end{array}$ & $\begin{array}{c}\text { Peak } \\
\text { load } \\
\text { with } \\
\text { full } \\
\text { strength } \\
(\mathbf{k N}) \\
\end{array}$ & $\begin{array}{c}\text { Shear } \\
\text { Capacity } \\
\text { Ratio }\end{array}$ \\
\hline 1 & & 40 & 4000 & $100.0 \%$ & 7.759 & & \\
\hline 2 & 1.0 & 37 & 3700 & $92.5 \%$ & 3.027 & 31.04 & $36.1 \%$ \\
\hline 3 & 0.7 & 41 & 4100 & $95.3 \%$ & 4.491 & 33.36 & $55.2 \%$ \\
\hline 4 & 1.0 & 35 & 3360 & $92.1 \%$ & 7.353 & 28.30 & $87.3 \%$ \\
\hline 5 & 0.8 & 35 & 3500 & $87.5 \%$ & 4.413 & 31.04 & $49.8 \%$ \\
\hline 6 & 0.8 & 36 & 3600 & $87.8 \%$ & 4.162 & 31.81 & $47.1 \%$ \\
\hline 7 & 0.8 & 32 & 3200 & $80.0 \%$ & 5.001 & 31.04 & $51.6 \%$ \\
\hline 8 & 0.8 & 36 & 3600 & $81.8 \%$ & 4.118 & 34.14 & $43.4 \%$ \\
\hline 9 & 0.7 & 29 & 2871 & $72.5 \%$ & 3.066 & 30.73 & $28.7 \%$ \\
\hline 10 & 0.6 & 35 & 3500 & $79.5 \%$ & 3.853 & 34.14 & $39.5 \%$ \\
\hline 11 & 2.0 & 34 & 3400 & $85.0 \%$ & 5.314 & 31.04 & $58.2 \%$ \\
\hline 12 & 2.0 & 34 & 3400 & $85.0 \%$ & 3.056 & 31.04 & $33.5 \%$ \\
\hline 13 & 1.3 & 32 & 3200 & $80.0 \%$ & 2.154 & 31.04 & $22.2 \%$ \\
\hline 14 & 1.5 & 31 & 3100 & $77.5 \%$ & 4.033 & 31.04 & $40.3 \%$ \\
\hline 15 & 1.1 & 29 & 2900 & $72.5 \%$ & 5.743 & 31.04 & $53.7 \%$ \\
\hline 16 & 0.8 & 36 & 3564 & $81.8 \%$ & 2.725 & 33.80 & $28.7 \%$ \\
\hline 17 & 1.1 & 35 & 3500 & $76.1 \%$ & 5.074 & 35.69 & $49.8 \%$ \\
\hline 18 & 0.7 & 31 & 3131 & $73.8 \%$ & 2.830 & 32.91 & $26.9 \%$ \\
\hline 19 & 0.9 & 31 & 3100 & $68.9 \%$ & 2.246 & 34.92 & $19.9 \%$ \\
\hline
\end{tabular}




\begin{tabular}{|c|c|c|c|c|c|}
\hline \multirow[b]{2}{*}{ Sample } & \multicolumn{2}{|c|}{$\begin{array}{c}\text { Calculated Zero Strength } \\
\text { Layer }\end{array}$} & \multicolumn{3}{|c|}{$\begin{array}{c}\text { Calculated Remaining Shear } \\
\text { Areas with Zero Strength } \\
\text { Layer increased by calculated } \\
\text { average }\end{array}$} \\
\hline & $\begin{array}{l}\text { Unheated } \\
\text { thickness at } \\
\text { full strength } \\
\quad(\mathbf{m m})\end{array}$ & $\begin{array}{l}\text { Difference } \\
\text { from } \\
\text { thickness } \\
\text { below char } \\
\text { layer (mm) }\end{array}$ & $\begin{array}{l}\text { New } \\
\text { "Char" } \\
\text { Depth } \\
\text { (mm) }\end{array}$ & $\begin{array}{l}\text { Remaining } \\
\text { Shear } \\
\text { Area } \\
\left(\mathbf{m m}^{2}\right)\end{array}$ & $\begin{array}{l}\text { Shear } \\
\text { Area } \\
\text { Ratio }\end{array}$ \\
\hline \multicolumn{6}{|l|}{1} \\
\hline 2 & 14.4 & -22.6 & 19.16 & 2083.78 & $52 \%$ \\
\hline 3 & 23.7 & -17.3 & 18.16 & 2483.78 & $58 \%$ \\
\hline 4 & 33.2 & -1.8 & 19.16 & 1808.43 & $50 \%$ \\
\hline 5 & 19.9 & -15.1 & 21.16 & 1883.78 & $47 \%$ \\
\hline 6 & 19.3 & -16.7 & 21.16 & 1983.78 & $48 \%$ \\
\hline 7 & 20.6 & -11.4 & 24.16 & 1583.78 & $40 \%$ \\
\hline 8 & 19.1 & -16.9 & 24.16 & 1983.78 & $45 \%$ \\
\hline 9 & 11.5 & -17.5 & 27.16 & 1270.94 & $32 \%$ \\
\hline 10 & 17.4 & -17.6 & 25.16 & 1883.78 & $43 \%$ \\
\hline 11 & 23.3 & -10.7 & 22.16 & 1783.78 & $45 \%$ \\
\hline 12 & 13.4 & -20.6 & 22.16 & 1783.78 & $45 \%$ \\
\hline 13 & 8.9 & -23.1 & 24.16 & 1583.78 & $40 \%$ \\
\hline 14 & 16.1 & -14.9 & 25.16 & 1483.78 & $37 \%$ \\
\hline 15 & 21.5 & -7.5 & 27.16 & 1283.78 & $32 \%$ \\
\hline 16 & 12.6 & -23.4 & 24.16 & 1963.94 & $45 \%$ \\
\hline 17 & 22.9 & -12.1 & 27.16 & 1883.78 & $41 \%$ \\
\hline 18 & 11.3 & -19.7 & 27.16 & 1498.62 & $35 \%$ \\
\hline \multirow[t]{2}{*}{19} & 9.0 & -22.0 & 30.16 & 1483.78 & $33 \%$ \\
\hline & AVG & -16.2 & & & \\
\hline
\end{tabular}




\begin{tabular}{|c|c|c|c|c|c|c|}
\hline \multirow[b]{2}{*}{ Sample } & \multicolumn{3}{|c|}{$\begin{array}{l}\text { Calculated Remaining Shear } \\
\text { Areas with Zero Strength } \\
\text { Layer increased by } 16 \mathrm{~mm} \\
\text { (intermediate) }\end{array}$} & \multicolumn{3}{|c|}{$\begin{array}{l}\text { Calculated Remaining Shear } \\
\text { Areas with Zero Strength } \\
\text { Layer increased by } 23 \mathrm{~mm} \\
\left(95^{\text {th }} \text { percentile }\right)\end{array}$} \\
\hline & $\begin{array}{l}\text { New } \\
\text { "Char" } \\
\text { Depth } \\
\text { (mm) }\end{array}$ & $\begin{array}{l}\text { Remaining } \\
\text { Shear } \\
\text { Area } \\
(\mathbf{m m 2})\end{array}$ & $\begin{array}{l}\text { Shear } \\
\text { Area } \\
\text { Ratio }\end{array}$ & $\begin{array}{l}\text { New } \\
\text { "Char" } \\
\text { Depth } \\
\text { (mm) }\end{array}$ & $\begin{array}{l}\text { Remaining } \\
\text { Shear } \\
\text { Area } \\
(\mathrm{mm} 2)\end{array}$ & $\begin{array}{l}\text { Shear } \\
\text { Area } \\
\text { Ratio }\end{array}$ \\
\hline \multicolumn{7}{|l|}{1} \\
\hline 2 & 19.00 & 2100.00 & $53 \%$ & 26.00 & 1400.00 & $35 \%$ \\
\hline 3 & 18.00 & 2500.00 & $58 \%$ & 25.00 & 1800.00 & $42 \%$ \\
\hline 4 & 19.00 & 1824.00 & $50 \%$ & 26.00 & 1152.00 & $32 \%$ \\
\hline 5 & 21.00 & 1900.00 & $48 \%$ & 28.00 & 1200.00 & $30 \%$ \\
\hline 6 & 21.00 & 2000.00 & $49 \%$ & 28.00 & 1300.00 & $32 \%$ \\
\hline 7 & 24.00 & 1600.00 & $40 \%$ & 31.00 & 900.00 & $23 \%$ \\
\hline 8 & 24.00 & 2000.00 & $45 \%$ & 31.00 & 1300.00 & $30 \%$ \\
\hline 9 & 27.00 & 1287.00 & $33 \%$ & 34.00 & 594.00 & $15 \%$ \\
\hline 10 & 25.00 & 1900.00 & $43 \%$ & 32.00 & 1200.00 & $27 \%$ \\
\hline 11 & 22.00 & 1800.00 & $45 \%$ & 29.00 & 1100.00 & $28 \%$ \\
\hline 12 & 22.00 & 1800.00 & $45 \%$ & 29.00 & 1100.00 & $28 \%$ \\
\hline 13 & 24.00 & 1600.00 & $40 \%$ & 31.00 & 900.00 & $23 \%$ \\
\hline 14 & 25.00 & 1500.00 & $38 \%$ & 32.00 & 800.00 & $20 \%$ \\
\hline 15 & 27.00 & 1300.00 & $33 \%$ & 34.00 & 600.00 & $15 \%$ \\
\hline 16 & 24.00 & 1980.00 & $45 \%$ & 31.00 & 1287.00 & $30 \%$ \\
\hline 17 & 27.00 & 1900.00 & $41 \%$ & 34.00 & 1200.00 & $26 \%$ \\
\hline 18 & 27.00 & 1515.00 & $36 \%$ & 34.00 & 808.00 & $19 \%$ \\
\hline 19 & 30.00 & 1500.00 & $33 \%$ & 37.00 & 800.00 & $18 \%$ \\
\hline
\end{tabular}




\begin{tabular}{|c|c|c|c|c|c|c|}
\hline & \multirow{2}{*}{\multicolumn{6}{|c|}{$\begin{array}{l}\text { Sacrificial char method - code prediction versus calculated } \mathbf{9 5}^{\text {th }} \text { percentile zero } \\
\text { strength layer } \\
\text { *Charring rate based on standard fire - NOT applicable for } 30 \& 50 \mathrm{~kW} / \mathrm{m}^{2} \text { heat } \\
\text { exposure }\end{array}$}} \\
\hline & & & & & & \\
\hline & Rate & 0.7 & \multirow{2}{*}{$\begin{array}{c}\mathrm{mm} / \mathrm{min} \\
\text { Predicted } \\
\text { Remaining } \\
\text { Shear } \\
\text { Area } \\
\left(\mathbf{m m}^{2}\right)\end{array}$} & \multicolumn{3}{|c|}{$+7 \mathrm{~mm}$ zero strength (code recommend) } \\
\hline Sample & $\begin{array}{c}\text { Predicted } \\
\text { Char } \\
\text { Depth } \\
(\mathbf{m m})\end{array}$ & $\begin{array}{l}\text { Difference } \\
\quad(\mathbf{m m})\end{array}$ & & $\begin{array}{l}\text { Predicted } \\
\text { Remaining } \\
\text { Capacity } \\
\quad(\mathbf{k N})\end{array}$ & $\begin{array}{l}\text { Difference } \\
\text { in Capacity } \\
\quad(\mathbf{k N})\end{array}$ & $\%$ Difference \\
\hline \multicolumn{7}{|l|}{1} \\
\hline 2 & 9.1 & -6.1 & 3090 & 23.98 & -12.78 & $-53.3 \%$ \\
\hline 3 & 9.1 & -7.1 & 3390 & 26.30 & -7.89 & $-30.0 \%$ \\
\hline 4 & 9.1 & -6.1 & 2774.4 & 21.53 & 3.18 & $14.8 \%$ \\
\hline 5 & 11.2 & -6.2 & 2880 & 22.35 & -6.90 & $-30.9 \%$ \\
\hline 6 & 11.2 & -6.2 & 2980 & 23.12 & -8.14 & $-35.2 \%$ \\
\hline 7 & 14 & -6 & 2600 & 20.17 & -4.17 & $-20.7 \%$ \\
\hline 8 & 14 & -6 & 3000 & 23.28 & -8.45 & $-36.3 \%$ \\
\hline 9 & 17.5 & -6.5 & 2227.5 & 17.28 & -8.48 & $-49.1 \%$ \\
\hline 10 & 17.5 & -8.5 & 2650 & 20.56 & -7.07 & $-34.4 \%$ \\
\hline 11 & 9.1 & -3.1 & 3090 & 23.98 & -5.91 & $-24.6 \%$ \\
\hline 12 & 9.1 & -3.1 & 3090 & 23.98 & -13.58 & $-56.7 \%$ \\
\hline 13 & 11.2 & -3.2 & 2880 & 22.35 & -15.45 & $-69.2 \%$ \\
\hline 14 & 11.2 & -2.2 & 2880 & 22.35 & -9.84 & $-44.1 \%$ \\
\hline 15 & 14 & -3 & 2600 & 20.17 & -3.52 & $-17.4 \%$ \\
\hline 16 & 14 & -6 & 2970 & 23.04 & -13.33 & $-57.9 \%$ \\
\hline 17 & 14 & -3 & 3200 & 24.83 & -7.07 & $-28.5 \%$ \\
\hline 18 & 17.5 & -6.5 & 2474.5 & 19.20 & -10.34 & $-53.8 \%$ \\
\hline 19 & 17.5 & -3.5 & 2750 & 21.34 & -14.37 & $-67.4 \%$ \\
\hline & & & & AVG & -8.56 & $-39 \%$ \\
\hline
\end{tabular}




\begin{tabular}{|c|c|c|c|c|c|c|}
\hline & \multirow{2}{*}{\multicolumn{6}{|c|}{$\begin{array}{l}\text { Sacrificial char method - code prediction versus calculated } \mathbf{9 5}^{\text {th }} \text { percentile zero } \\
\text { strength layer } \\
{ }^{*} \text { Charring rate based on standard fire - NOT applicable for } 30 \& 50 \mathrm{~kW} / \mathrm{m}^{2} \text { heat } \\
\text { exposure }\end{array}$}} \\
\hline & & & & & & \\
\hline & Rate & 0.7 & \multirow[b]{2}{*}{$\begin{array}{c}\mathrm{mm} / \mathrm{min} \\
\text { Predicted } \\
\text { Remaining } \\
\text { Shear } \\
\text { Area } \\
\left(\mathbf{m m}^{2}\right) \\
\end{array}$} & \multicolumn{3}{|c|}{$+23 \mathrm{~mm}$ zero strength $\left(95^{\text {th }}\right.$ percentile) } \\
\hline Sample & $\begin{array}{l}\text { Predicted } \\
\text { Char } \\
\text { Depth } \\
(\mathbf{m m})\end{array}$ & $\begin{array}{l}\text { Difference } \\
\quad(\mathrm{mm})\end{array}$ & & $\begin{array}{l}\text { Predicted } \\
\text { Remaining } \\
\text { Capacity } \\
\quad(k N)\end{array}$ & $\begin{array}{l}\text { Difference } \\
\text { in } \\
\text { Capacity } \\
\text { (kN) }\end{array}$ & $\begin{array}{c}\% \\
\text { Difference }\end{array}$ \\
\hline \multicolumn{7}{|l|}{1} \\
\hline 2 & 25.1 & -22.1 & 1490.0 & 11.56 & -0.36 & $-3 \%$ \\
\hline 3 & 25.1 & -23.1 & 1790.0 & 13.89 & 4.53 & $33 \%$ \\
\hline 4 & 25.1 & -22.1 & 1238.4 & 9.61 & 15.10 & $157 \%$ \\
\hline 5 & 27.2 & -22.2 & 1280.0 & 9.93 & 5.51 & $56 \%$ \\
\hline 6 & 27.2 & -22.2 & 1380.0 & 10.71 & 4.27 & $40 \%$ \\
\hline 7 & 30.0 & -22 & 1000.0 & 7.76 & 8.25 & $106 \%$ \\
\hline 8 & 30.0 & -22 & 1400.0 & 10.86 & 3.96 & $36 \%$ \\
\hline 9 & 33.5 & -22.5 & 643.5 & 4.99 & 3.81 & $76 \%$ \\
\hline 10 & 33.5 & -24.5 & 1050.0 & 8.15 & 5.34 & $66 \%$ \\
\hline 11 & 25.1 & -19.1 & 1490.0 & 11.56 & 6.51 & $56 \%$ \\
\hline 12 & 25.1 & -19.1 & 1490.0 & 11.56 & -1.17 & $-10 \%$ \\
\hline 13 & 27.2 & -19.2 & 1280.0 & 9.93 & -3.04 & $-31 \%$ \\
\hline 14 & 27.2 & -18.2 & 1280.0 & 9.93 & 2.57 & $26 \%$ \\
\hline 15 & 30.0 & -19 & 1000.0 & 7.76 & 8.90 & $115 \%$ \\
\hline 16 & 30.0 & -22 & 1386.0 & 10.75 & -1.04 & $-10 \%$ \\
\hline 17 & 30.0 & -19 & 1600.0 & 12.41 & 5.34 & $43 \%$ \\
\hline 18 & 33.5 & -22.5 & 858.5 & 6.66 & 2.20 & $33 \%$ \\
\hline 19 & 33.5 & -19.5 & 1150.0 & 8.92 & -1.96 & $-22 \%$ \\
\hline & & & & AVG & 3.82 & $43 \%$ \\
\hline
\end{tabular}




\section{D.2 PUR Samples}

\begin{tabular}{|c|c|c|c|c|c|c|c|c|}
\hline \multicolumn{7}{|c|}{ Sample Information } & \multicolumn{2}{|c|}{ Recorded Data } \\
\hline Sample & Type & $\begin{array}{l}\text { Heating } \\
\left(\mathbf{k W} / \mathbf{m}^{2}\right)\end{array}$ & $\begin{array}{c}\text { Duration } \\
\text { (min) }\end{array}$ & $\begin{array}{c}\text { Original } \\
\text { Thickness } \\
\quad(\mathbf{m m})\end{array}$ & $\begin{array}{c}\text { Sample } \\
\text { Height } \\
\text { (mm) }\end{array}$ & $\begin{array}{c}\text { Original } \\
\text { Shear } \\
\text { Area } \\
\left(\mathbf{m m}^{2}\right) \\
\end{array}$ & $\begin{array}{c}\text { Peak } \\
\text { Load } \\
(k N)\end{array}$ & $\begin{array}{l}\text { Char } \\
\text { depth } \\
(\mathrm{mm})\end{array}$ \\
\hline 20 & PUR & 0 & 0 & 40 & 100 & 4000 & 32.45 & 0 \\
\hline 21 & PUR & 0 & 0 & 40 & 100 & 4000 & 39.17 & 0 \\
\hline AVG & PUR & $\mathbf{0}$ & $\mathbf{0}$ & & & & 35.81 & $\mathbf{0}$ \\
\hline 22 & PUR & 30 & 3 & 40 & 100 & 4000 & 27.07 & 2 \\
\hline 23 & PUR & 30 & 3 & 40 & 100 & 4000 & 31.43 & 3 \\
\hline 24 & PUR & 30 & 3 & 41 & 99 & 4059 & 18.14 & 2 \\
\hline 25 & PUR & 30 & 6 & 40 & 100 & 4000 & 22.55 & 5 \\
\hline 26 & PUR & 30 & 6 & 40 & 100 & 4000 & 16.14 & 5 \\
\hline 27 & PUR & 30 & 6 & 41 & 97 & 3977 & 27.88 & 4 \\
\hline 28 & PUR & 30 & 10 & 40 & 100 & 4000 & 26.09 & 7 \\
\hline 29 & PUR & 30 & 10 & 40 & 100 & 4000 & 16.15 & 9 \\
\hline 30 & PUR & 30 & 10 & 40 & 99 & 3960 & 18.57 & 8 \\
\hline 31 & PUR & 30 & 10 & 40 & 99 & 3960 & 22.52 & 6 \\
\hline 32 & PUR & 30 & 15 & 40 & 100 & 4000 & 13.76 & 13 \\
\hline 33 & PUR & 30 & 15 & 40 & 100 & 4000 & 14.21 & 13 \\
\hline 34 & PUR & 30 & 15 & 41 & 99 & 4059 & 25.90 & 11 \\
\hline 35 & PUR & 30 & 15 & 43 & 100 & 4300 & 18.41 & 9 \\
\hline 36 & PUR & 50 & 3 & 40 & 100 & 4000 & 18.81 & 5 \\
\hline 37 & PUR & 50 & 3 & 40 & 100 & 4000 & 15.20 & 5 \\
\hline 38 & PUR & 50 & 6 & 40 & 100 & 4000 & 13.57 & 9 \\
\hline 39 & PUR & 50 & 6 & 40 & 100 & 4000 & 11.36 & 9 \\
\hline 40 & PUR & 50 & 10 & 40 & 100 & 4000 & 24.62 & 11 \\
\hline 41 & PUR & 50 & 10 & 40 & 100 & 4000 & 28.50 & 11 \\
\hline 42 & PUR & 50 & 10 & 44 & 100 & 4400 & 8.716 & 10 \\
\hline 43 & PUR & 50 & 10 & 45 & 98 & 4410 & 24.03 & 12 \\
\hline 44 & PUR & 50 & 15 & 40 & 100 & 4000 & 12.72 & 14 \\
\hline 45 & PUR & 50 & 15 & 40 & 100 & 4000 & 10.86 & 14 \\
\hline 46 & PUR & 50 & 15 & 44 & 97 & 4268 & 16.12 & 12 \\
\hline
\end{tabular}




\begin{tabular}{|c|c|c|c|c|c|c|c|}
\hline & \multicolumn{4}{|c|}{ Char Calculations } & \multicolumn{3}{|c|}{ Strength Calculations } \\
\hline Sample & $\begin{array}{c}\text { Averaged } \\
\text { Charring } \\
\text { Rate } \\
(\mathbf{m m} / \mathbf{m i n})\end{array}$ & $\begin{array}{c}\text { Thickness } \\
\text { below } \\
\text { char } \\
\text { layer } \\
(\mathbf{m m})\end{array}$ & $\begin{array}{c}\text { Remaining } \\
\text { Shear } \\
\text { Area } \\
\left(\mathbf{m m}^{2}\right)\end{array}$ & $\begin{array}{l}\text { Shear } \\
\text { Area } \\
\text { Ratio }\end{array}$ & $\begin{array}{l}\text { Failure } \\
\text { Stress } \\
\text { (MPa) }\end{array}$ & $\begin{array}{c}\text { Peak } \\
\text { load } \\
\text { with } \\
\text { full } \\
\text { strength } \\
(\mathbf{k N}) \\
\end{array}$ & $\begin{array}{c}\text { Shear } \\
\text { Capacity } \\
\text { Ratio }\end{array}$ \\
\hline 20 & & 40 & 4000 & $100.0 \%$ & 8.113 & & \\
\hline 21 & & 40 & 4000 & $100.0 \%$ & 9.793 & & \\
\hline AVG & & & & & 8.953 & & \\
\hline 22 & 0.7 & 38 & 3800 & $95.0 \%$ & 7.126 & 35.81 & $75.6 \%$ \\
\hline 23 & 1.0 & 37 & 3700 & $92.5 \%$ & 8.495 & 35.81 & $87.8 \%$ \\
\hline 24 & 0.7 & 39 & 3861 & $95.1 \%$ & 4.700 & 36.34 & $49.9 \%$ \\
\hline 25 & 0.8 & 35 & 3500 & $87.5 \%$ & 6.444 & 35.81 & $63.0 \%$ \\
\hline 26 & 0.8 & 35 & 3500 & $87.5 \%$ & 4.613 & 35.81 & $45.1 \%$ \\
\hline 27 & 0.7 & 37 & 3589 & $90.2 \%$ & 7.769 & 35.61 & $78.3 \%$ \\
\hline 28 & 0.7 & 33 & 3300 & $82.5 \%$ & 7.909 & 35.81 & $72.9 \%$ \\
\hline 29 & 0.9 & 31 & 3100 & $77.5 \%$ & 5.212 & 35.81 & $45.1 \%$ \\
\hline 30 & 0.8 & 32 & 3168 & $80.0 \%$ & 5.862 & 35.45 & $52.4 \%$ \\
\hline 31 & 0.6 & 34 & 3366 & $85.0 \%$ & 6.690 & 35.45 & $63.5 \%$ \\
\hline 32 & 0.9 & 27 & 2700 & $67.5 \%$ & 5.097 & 35.81 & $38.4 \%$ \\
\hline 33 & 0.9 & 27 & 2700 & $67.5 \%$ & 5.266 & 35.81 & $39.7 \%$ \\
\hline 34 & 0.7 & 30 & 2970 & $73.2 \%$ & 8.723 & 36.34 & $71.3 \%$ \\
\hline 35 & 0.6 & 34 & 3400 & $79.1 \%$ & 5.416 & 38.50 & $47.8 \%$ \\
\hline 36 & 1.7 & 35 & 3500 & $87.5 \%$ & 5.375 & 35.81 & $52.5 \%$ \\
\hline 37 & 1.7 & 35 & 3500 & $87.5 \%$ & 4.345 & 35.81 & $42.5 \%$ \\
\hline 38 & 1.5 & 31 & 3100 & $77.5 \%$ & 4.379 & 35.81 & $37.9 \%$ \\
\hline 39 & 1.5 & 31 & 3100 & $77.5 \%$ & 3.666 & 35.81 & $31.7 \%$ \\
\hline 40 & 1.1 & 29 & 2900 & $72.5 \%$ & 8.492 & 35.81 & $68.8 \%$ \\
\hline 41 & 1.1 & 29 & 2900 & $72.5 \%$ & 9.829 & 35.81 & $79.6 \%$ \\
\hline 42 & 1.0 & 34 & 3400 & $77.3 \%$ & 2.564 & 39.39 & $22.1 \%$ \\
\hline 43 & 1.2 & 33 & 3234 & $73.3 \%$ & 7.431 & 39.48 & $60.9 \%$ \\
\hline 44 & 0.9 & 26 & 2600 & $65.0 \%$ & 4.892 & 35.81 & $35.5 \%$ \\
\hline 45 & 0.9 & 26 & 2600 & $65.0 \%$ & 4.180 & 35.81 & $30.3 \%$ \\
\hline 46 & 0.8 & 32 & 3104 & $72.7 \%$ & 5.194 & 38.21 & $42.2 \%$ \\
\hline
\end{tabular}




\begin{tabular}{|c|c|c|c|c|c|}
\hline & \multicolumn{2}{|c|}{ Calculated Zero Strength Layer } & \multicolumn{3}{|c|}{$\begin{array}{c}\text { Calculated Remaining Shear } \\
\text { Areas with Zero Strength } \\
\text { Layer increased by calculated } \\
\text { average }\end{array}$} \\
\hline Sample & $\begin{array}{c}\text { Unheated } \\
\text { thickness at full } \\
\text { strength }(\mathbf{m m})\end{array}$ & $\begin{array}{c}\text { Difference } \\
\text { from } \\
\text { thickness } \\
\text { below char } \\
\text { layer (mm) }\end{array}$ & $\begin{array}{l}\text { New } \\
\text { "Char" } \\
\text { Depth } \\
(\mathbf{m m})\end{array}$ & $\begin{array}{l}\text { Remaining } \\
\text { Shear } \\
\text { Area } \\
\left(\mathbf{m m}^{2}\right)\end{array}$ & $\begin{array}{l}\text { Shear } \\
\text { Area } \\
\text { Ratio }\end{array}$ \\
\hline $\begin{array}{r}20 \\
21 \\
\text { AVG }^{2} \\
\end{array}$ & & & & & \\
\hline 22 & 30.2 & -7.8 & 12.68 & 2731.98 & $68 \%$ \\
\hline 23 & 35.1 & -1.9 & 13.68 & 2631.98 & $66 \%$ \\
\hline 24 & 20.5 & -18.5 & 12.68 & 2803.66 & $69 \%$ \\
\hline 25 & 25.2 & -9.8 & 15.68 & 2431.98 & $61 \%$ \\
\hline 26 & 18.0 & -17.0 & 15.68 & 2431.98 & $61 \%$ \\
\hline 27 & 32.1 & -4.9 & 14.68 & 2553.02 & $64 \%$ \\
\hline 28 & 29.2 & -3.8 & 17.68 & 2231.98 & $56 \%$ \\
\hline 29 & 18.0 & -13.0 & 19.68 & 2031.98 & $51 \%$ \\
\hline 30 & 21.0 & -11.0 & 18.68 & 2110.66 & $53 \%$ \\
\hline 31 & 25.4 & -8.6 & 16.68 & 2308.66 & $58 \%$ \\
\hline 32 & 15.4 & -11.6 & 23.68 & 1631.98 & $41 \%$ \\
\hline 33 & 15.9 & -11.1 & 23.68 & 1631.98 & $41 \%$ \\
\hline 34 & 29.2 & -0.8 & 21.68 & 1912.66 & $47 \%$ \\
\hline 35 & 20.6 & -13.4 & 19.68 & 2331.98 & $54 \%$ \\
\hline 36 & 21.0 & -14.0 & 15.68 & 2431.98 & $61 \%$ \\
\hline 37 & 17.0 & -18.0 & 15.68 & 2431.98 & $61 \%$ \\
\hline 38 & 15.2 & -15.8 & 19.68 & 2031.98 & $51 \%$ \\
\hline 39 & 12.7 & -18.3 & 19.68 & 2031.98 & $51 \%$ \\
\hline 40 & 27.5 & -1.5 & 21.68 & 1831.98 & $46 \%$ \\
\hline 41 & 31.8 & 2.8 & 21.68 & 1831.98 & $46 \%$ \\
\hline 42 & 9.7 & -24.3 & 20.68 & 2331.98 & $53 \%$ \\
\hline 43 & 27.4 & -5.6 & 22.68 & 2187.34 & $50 \%$ \\
\hline 44 & 14.2 & -11.8 & 24.68 & 1531.98 & $38 \%$ \\
\hline 45 & 12.1 & -13.9 & 24.68 & 1531.98 & $38 \%$ \\
\hline 46 & 18.6 & -13.4 & 22.68 & 2068.02 & $48 \%$ \\
\hline & AVG & -10.68 & & & \\
\hline
\end{tabular}




\begin{tabular}{|c|c|c|c|c|c|c|}
\hline \multirow[b]{2}{*}{ Sample } & \multicolumn{3}{|c|}{$\begin{array}{l}\text { Calculated Remaining Shear Areas } \\
\text { with Zero Strength Layer increased } \\
\text { by } 16 \mathrm{~mm} \text { (intermediate) }\end{array}$} & \multicolumn{3}{|c|}{$\begin{array}{c}\text { Calculated Remaining Shear } \\
\text { Areas with Zero Strength Layer } \\
\text { increased by } 23 \mathrm{~mm}(95 \mathrm{th} \\
\text { percentile) }\end{array}$} \\
\hline & $\begin{array}{l}\text { New "Char" } \\
\text { Depth (mm) }\end{array}$ & $\begin{array}{l}\text { Remaining } \\
\text { Shear } \\
\text { Area } \\
\text { (mm2) }\end{array}$ & $\begin{array}{l}\text { Shear } \\
\text { Area } \\
\text { Ratio }\end{array}$ & $\begin{array}{l}\text { New } \\
\text { "Char" } \\
\text { Depth } \\
\text { (mm) }\end{array}$ & $\begin{array}{c}\text { Remaining } \\
\text { Shear } \\
\text { Area } \\
(\mathbf{m m 2})\end{array}$ & $\begin{array}{l}\text { Shear } \\
\text { Area } \\
\text { Ratio }\end{array}$ \\
\hline $\begin{array}{r}20 \\
21 \\
\text { AVG }^{21}\end{array}$ & & & & & & \\
\hline 22 & 18.00 & 2200.00 & $55 \%$ & 25.00 & 1500.00 & $38 \%$ \\
\hline 23 & 19.00 & 2100.00 & $53 \%$ & 26.00 & 1400.00 & $35 \%$ \\
\hline 24 & 18.00 & 2277.00 & $56 \%$ & 25.00 & 1584.00 & $39 \%$ \\
\hline 25 & 21.00 & 1900.00 & $48 \%$ & 28.00 & 1200.00 & $30 \%$ \\
\hline 26 & 21.00 & 1900.00 & $48 \%$ & 28.00 & 1200.00 & $30 \%$ \\
\hline 27 & 20.00 & 2037.00 & $51 \%$ & 27.00 & 1358.00 & $34 \%$ \\
\hline 28 & 23.00 & 1700.00 & $43 \%$ & 30.00 & 1000.00 & $25 \%$ \\
\hline 29 & 25.00 & 1500.00 & $38 \%$ & 32.00 & 800.00 & $20 \%$ \\
\hline 30 & 24.00 & 1584.00 & $40 \%$ & 31.00 & 891.00 & $23 \%$ \\
\hline 31 & 22.00 & 1782.00 & $45 \%$ & 29.00 & 1089.00 & $28 \%$ \\
\hline 32 & 29.00 & 1100.00 & $28 \%$ & 36.00 & 400.00 & $10 \%$ \\
\hline 33 & 29.00 & 1100.00 & $28 \%$ & 36.00 & 400.00 & $10 \%$ \\
\hline 34 & 27.00 & 1386.00 & $34 \%$ & 34.00 & 693.00 & $17 \%$ \\
\hline 35 & 25.00 & 1800.00 & $42 \%$ & 32.00 & 1100.00 & $26 \%$ \\
\hline 36 & 21.00 & 1900.00 & $48 \%$ & 28.00 & 1200.00 & $30 \%$ \\
\hline 37 & 21.00 & 1900.00 & $48 \%$ & 28.00 & 1200.00 & $30 \%$ \\
\hline 38 & 25.00 & 1500.00 & $38 \%$ & 32.00 & 800.00 & $20 \%$ \\
\hline 39 & 25.00 & 1500.00 & $38 \%$ & 32.00 & 800.00 & $20 \%$ \\
\hline 40 & 27.00 & 1300.00 & $33 \%$ & 34.00 & 600.00 & $15 \%$ \\
\hline 41 & 27.00 & 1300.00 & $33 \%$ & 34.00 & 600.00 & $15 \%$ \\
\hline 42 & 26.00 & 1800.00 & $41 \%$ & 33.00 & 1100.00 & $25 \%$ \\
\hline 43 & 28.00 & 1666.00 & $38 \%$ & 35.00 & 980.00 & $22 \%$ \\
\hline 44 & 30.00 & 1000.00 & $25 \%$ & 37.00 & 300.00 & $8 \%$ \\
\hline 45 & 30.00 & 1000.00 & $25 \%$ & 37.00 & 300.00 & $8 \%$ \\
\hline 46 & 28.00 & 1552.00 & $36 \%$ & 35.00 & 873.00 & $20 \%$ \\
\hline
\end{tabular}




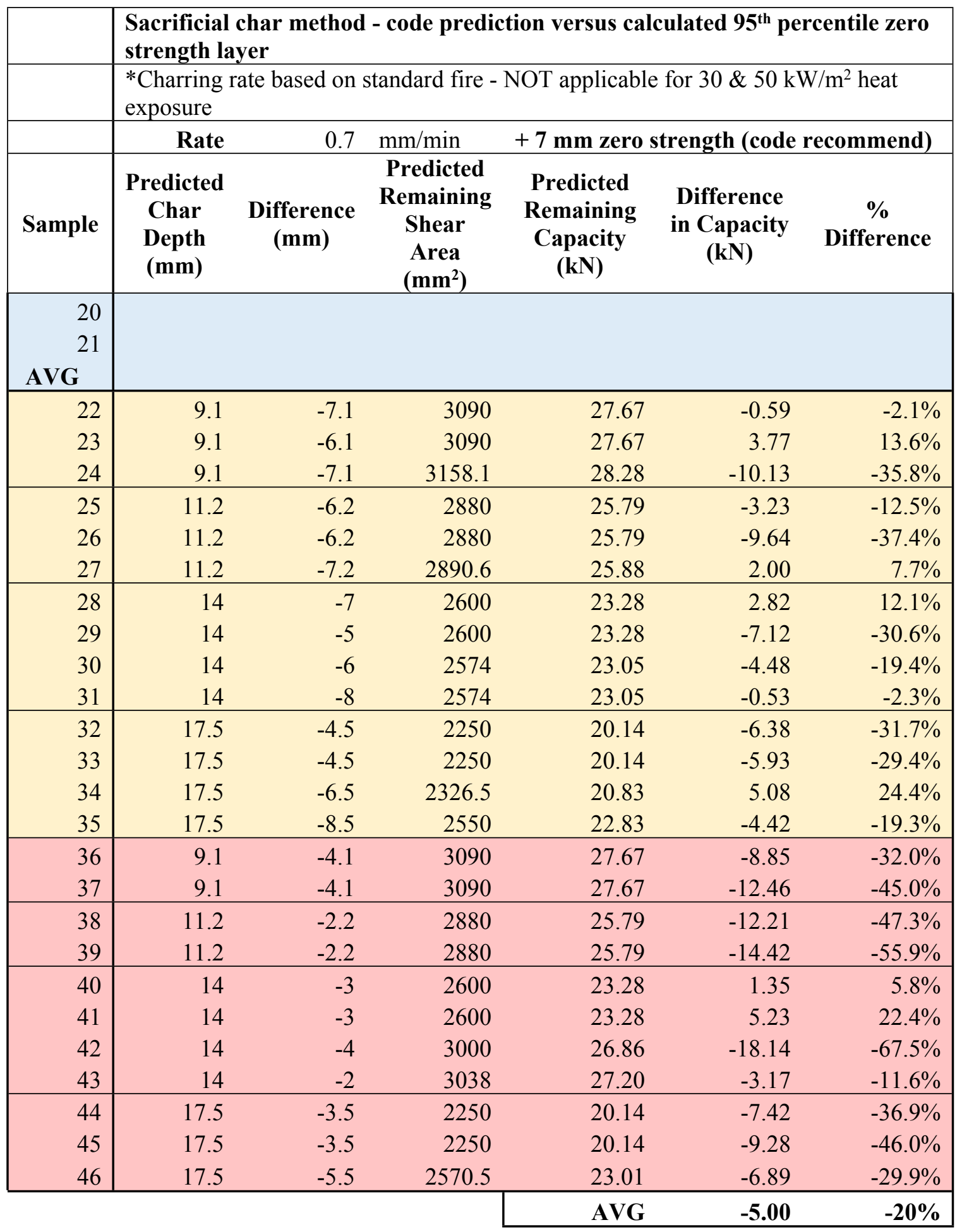




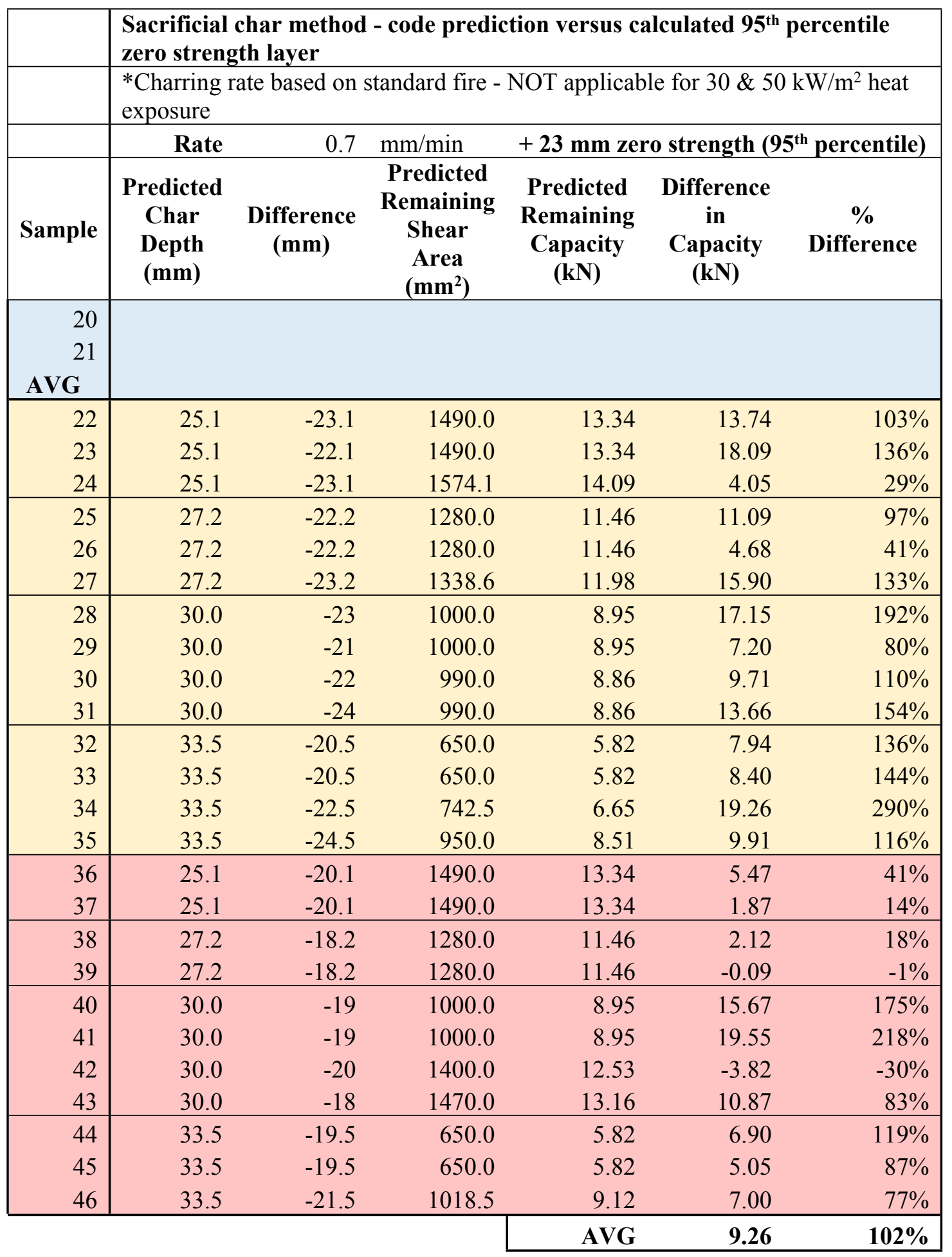

